\title{
Modelos GAS com distribuições estáveis para séries temporais financeiras
}

\author{
Daniel Takata Gomes
}

TESE APRESENTADA

$\mathrm{AO}$

Instituto de Matemática e Estatística

DA

UNIVERSIDADE DE SÃo PAUlo

PARA

OBTENÇÃO DO TÍTULO

$\mathrm{DE}$

DOUTOR EM CIÊNCIAS

\author{
Programa: Estatística \\ Orientadora: Prof ${ }^{\mathrm{a}}$. Dr ${ }^{\mathrm{a}}$. Chang Chiann

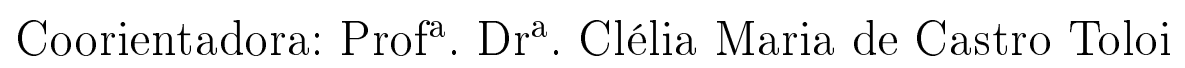




\title{
Modelos GAS com distribuições estáveis para séries temporais financeiras
}

\begin{abstract}
Esta versão da tese contém as correções e alterações sugeridas pela Comissão Julgadora durante a defesa da versão original do trabalho, realizada em 06/12/2017. Uma cópia da versão original está disponível no Instituto de Matemática e Estatística da Universidade de São Paulo.
\end{abstract}

Comissão Julgadora:

- Prof $^{\mathrm{a}}$. Dr ${ }^{\mathrm{a}}$. Chang Chiann (orientadora) - IME-USP

- Prof. Dr. Pedro Alberto Morettin - IME-USP

- Prof. Dr. Aluísio de Souza Pinheiro - UNICAMP

- Prof. Dr. Pedro Valls Pereira - FGV

- Prof. Dr. Cristiano Augusto Coelho Fernandes - PUC-RJ 
Durante o desenvolvimento deste trabalho o autor recebeu apoio e auxilio do IBGE, entidade na qual atua como professor e pesquisador. 


\section{Resumo}

Gomes, D. T. Modelos GAS com distribuições estáveis para séries temporais financeiras. 2017. 120 f. Tese (Doutorado) - Instituto de Matemática e Estatística, Universidade de São Paulo, São Paulo, 2017.

Modelos GARCH tendo a normal e a $t$-Student como distribuições condicionais são amplamente utilizados para modelagem da volatilidade de dados financeiros. No entanto, tais distribuições podem não ser apropriadas para algumas séries com caudas pesadas e comportamento leptocúrtico. As chamadas distribuições estáveis podem ser mais adequadas para sua modelagem, como já explorado na literatura. Por outro lado, os modelos GAS (Generalized Autoregressive Score), com desenvolvimento recente, tratam-se de modelos dinâmicos que possuem em sua estrutura a função score (derivada do logaritmo da verossimilhança). Tal abordagem oferece uma direção natural para a evolução dos parâmetros da distribuição dos dados. Neste trabalho, é proposto um novo modelo GAS em conjunção com distribuições estáveis simétricas para a modelagem da volatilidade - de fato, é uma generalização do GARCH, pois, para uma particular escolha de distribuição estável e de estrutura do modelo, tem-se o clássico modelo GARCH gaussiano. Como em geral a função densidade das distribuições estáveis não possui forma analítica fechada, é apresentado seu procedimento de cálculo, bem como de suas derivadas, para o completo desenvolvimento do método de estimação dos parâmetros. Também são analisadas as condições de estacionariedade e a estrutura de dependência do modelo. Estudos de simulação são conduzidos, bem como uma aplicação a dados reais, para comparação entre modelos usuais, que utilizam distribuições normal e $t$-Student, e o modelo proposto, demonstrando a eficácia deste.

Palavras-chave: heteroscedasticidade condicional, função score, distribuições estáveis, modelagem financeira, caudas pesadas. 


\section{Abstract}

Gomes, D. T. Stable GAS models for financial time series. 2017. 120 f. Tese (Doutorado) Instituto de Matemática e Estatística, Universidade de São Paulo, São Paulo, 2017.

GARCH models with normal and $t$-Student conditional distributions are widely used for volatility modeling in financial data. However, such distributions may not be suitable for some heavytailed and leptokurtic series. The stable distributions may be more adequate to fit such characteristics, as already exploited in the literature. On the other hand, the recently developed GAS (Generalized Autoregressive Score) models are dynamic models in which the updating mechanism of the time-varying parameters is based on the score function (first derivative of the log-likelihood function). This provides the natural direction for updating the parameters, based on the complete density. We propose a new GAS model with symmetric stable distribution for volatility modeling. The model can be interpreted as a generalization of the GARCH models, since the classic gaussian GARCH model is derived from it by using particular choices of the stable distribution and the model structure. There are no closed analytical expressions for general stable densities in most cases, hence its numeric computation and derivatives are detailed for the sake of complete development of the estimation process. The stationarity conditions and the dependence structure of the model are analysed. Simulation studies, as well as an application to real data, are presented for comparisons between the usual models and the proposed model, illustrating the effectiveness of the latter.

Keywords: conditional heteroscedasticity, score function, stable distributions, financial modeling, heavy tails. 


\section{Sumário}

$\begin{array}{ll}\text { Lista de Abreviaturas } & \text { ix }\end{array}$

Lista de Figuras

Lista de Tabelas $\quad$ xiii

1 Introdução $\quad 1$

2 Modelos GAS $\quad 3$

2.1 Especificação do modelo . . . . . . . . . . . . . . . . . . . . . 4

2.2 Modelos GAS para volatilidade . . . . . . . . . . . . . . . 6

2.3 Justificativa teórica para o uso do score . . . . . . . . . . . . . . . 8

2.4 Estacionariedade . . . . . . . . . . . . . . . . . . . 11

2.5 Estimação por máxima verossimilhança . . . . . . . . . . . . . . . . 13

3 Distribuições estáveis $\quad 15$

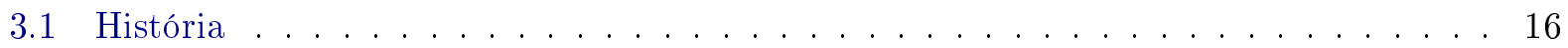

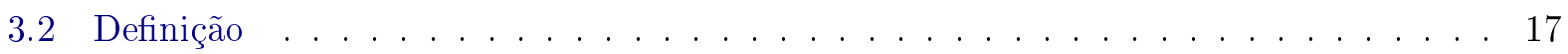

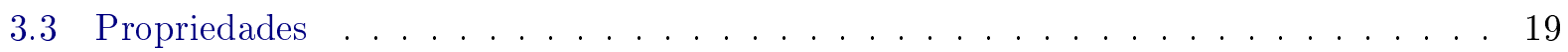

3.4 Densidades e funções distribuição . . . . . . . . . . . . . . . . . . . . 22

3.5 O Teorema Limite Central Generalizado . . . . . . . . . . . . . . . . . . . 23

3.6 Estrutura de dependência . . . . . . . . . . . . . . . . 24

3.7 Simulação . . . . . . . . . . . . . . . . . . . . 25

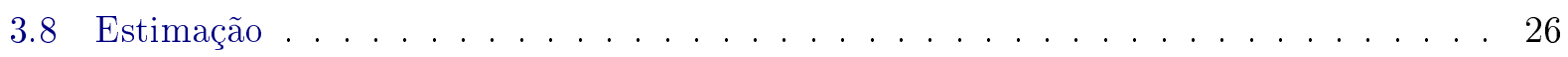

4 Cálculos referentes a distribuições estáveis simétricas $\quad 27$

4.1 Notação e resultados preliminares . . . . . . . . . . . . . . . . . . . . . 28

4.2 Derivadas parciais da função densidade . . . . . . . . . . . . . . 31

4.2.1 Primeira derivada em relação ao parâmetro de escala . . . . . . . . . . . . . 31

4.2.2 Segunda derivada em relação ao parâmetro de escala . . . . . . . . . . . . . . . . . . 32

4.2.3 Terceira derivada em relação ao parâmetro de escala . . . . . . . . . . . . . 33

4.2 .4 Primeira derivada com relação a $\alpha \ldots \ldots \ldots \ldots$. . . . . . . . . 34

4.2 .5 Segunda derivada em relação a $\alpha \ldots \ldots \ldots \ldots \ldots$

4.2 .6 Segunda derivada com relação a $\alpha$ e $\ldots \ldots \ldots \ldots \ldots \ldots$

4.3 Informação de Fisher e suas derivadas . . . . . . . . . . . . . . . . . . . . 37 
5 Modelo proposto

5.1 Modelos de volatilidade para dados com caudas pesadas . . . . . . . . . . . . 41

5.1 .1 Modelos GARCH clássicos . . . . . . . . . . . . . . . . . . 42

5.1 .2 Modelos GARCH com distribuições estáveis . . . . . . . . . . . . . . . . . 42

5.1.3 Modelos GAS com distribuição t-Student . . . . . . . . . . . . . . . . . . 44

5.2 Modelo proposto para volatilidade: GAS com distribuição estável . . . . . . . . . . 45

5.2 .1 Especificação do modelo . . . . . . . . . . . . . . . . . . 47

5.2 .2 Restrições para os parâmetros . . . . . . . . . . . . . . . . . 49

5.2 .3 Condições de estacionariedade . . . . . . . . . . . . . . . 50

5.2 .4 Estimação . . . . . . . . . . . . . . . . . . . 54

5.2 .5 Identificação do modelo . . . . . . . . . . . . . . . . . . . 57

6 Simulações e aplicação $\quad 63$

6.1 Simulações. . . . . . . . . . . . . . . . . . . . . . . 63

6.2 Aplicação . . . . . . . . . . . . . . . . . . . . . . . . 79

6.2 .1 Análise preliminar dos dados . . . . . . . . . . . . . . . . 79

6.2 .2 Modelagem . . . . . . . . . . . . . . . . . . . . 82

$\begin{array}{lll}7 & \text { Conclusões e trabalhos futuros } & 87\end{array}$

7.1 Considerações finais . . . . . . . . . . . . . . . . . 87

7.2 Sugestões para trabalhos futuros $\ldots \ldots \ldots \ldots \ldots$

$\begin{array}{lr}\text { A Códigos } & \mathbf{8 9}\end{array}$

A.1 Densidades de distribuições estáveis e suas derivadas . . . . . . . . . . . . . . . . 89

A.2 Informação de Fisher de distribuição estável padrão simétrica em relação ao parâmetro de escala . . . . . . . . . . . . . . . . . . . . . 95

A.3 Estimação dos parâmetros do modelo GAS com distribuição estável . . . . . . . . . 96

$\begin{array}{ll}\text { Referências Bibliográficas } & 99\end{array}$ 


\title{
Lista de Abreviaturas
}

\author{
ACD Autoregressive Conditional Duration \\ ACI Autoregressive Conditional Intensity \\ AD Anderson-Darling \\ ARCH Autoregressive Conditionally Heteroscedasticity \\ ARFIMA Autoregressive Fractionally Integrated Moving Average \\ CVM Cramér-von Mises \\ DCS Dynamic Conditional Score \\ EML Expoente Máximo de Lyapunov \\ FIGARCH Fractionally Integrated Generalized Autoregressive Conditionally Heteroscedasticity \\ GAS Generalized Autoregressive Score \\ GARCH Generalized Autoregressive Conditionally Heteroscedasticity \\ GED Distribuição Generalizada de Erros \\ MAE Erro Absoluto Médio \\ MEM Multiplicative Error Model
}




\section{Lista de Figuras}

3.1 Funções densidade de probabilidade de distribuições estáveis: normal ( $\alpha=2, \beta=0$, $\delta=0, \gamma=1)$, Cauchy $(\alpha=1, \beta=0, \delta=0, \gamma=1)$ e Lévy $(\alpha=1 / 2, \beta=1, \delta=0$,

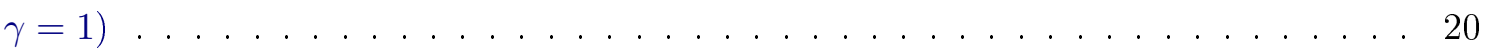

3.2 Comparação de funções densidade de distribuições estáveis em termos de variações de seus parâmetros . . . . . . . . . . . . . . . . . . 20

5.1 Funções densidade de probabilidade: distribuições estável $(\alpha=1,5)$, normal padrão e $t$-Student (7 graus de liberdade $) \ldots \ldots \ldots \ldots$. . . . . . . . . 41

5.2 Retornos diários de S\&P500 de 30 de outubro de 1985 a 24 de junho de 2016 . . . 46

5.3 Variância condicional dos retornos diários de S\&P500 estimada pelos modelos GARCH gaussiano, GARCH t-Student e GAS t-Student em trecho da série . . . . . . . . . . . 46

5.4 Funções densidade de distribuições normal e estável . . . . . . . . . . . . . . . . . . 48

5.5 Funções score de distribuições normal e estável . . . . . . . . . . . . . . . . . . . . . 49

5.6 Função de autocorrelação amostral para o quadrado de um processo GAS estável gerado com $\alpha=1,7, \omega=0,1, A_{1}=0,1$ e $B_{1}=0,9 \ldots \ldots \ldots \ldots$

5.7 Funções de codiferença normalizada amostrais do quadrado de simulações de processos GAS estáveis com $\alpha=1,7$ e $\omega=0,5 \ldots \ldots \ldots \ldots$. . . . . . . . 60

5.8 Funções de codiferença normalizada amostrais de simulações de processos GAS es-

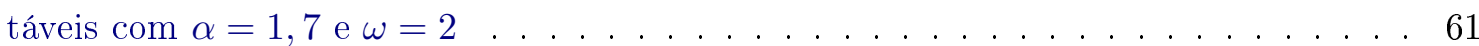

5.9 Funções de codiferença normalizada amostrais do quadrado de simulações de processos GAS estáveis com $\alpha=1,7$ e $\omega=0,01 \ldots \ldots \ldots$. . . . . . . . . 62

6.1 Séries geradas por diferentes processos com $\omega=0,5, A_{1}=0,1$ e $B_{1}=0,6 \ldots 64$

6.2 Box-plots das distribuições dos AICs corrigidos e dos BICs dos modelos ajustados para simulações de $\operatorname{GARCH}(1,1)$ gaussiano com $\alpha_{0}=0,1, \alpha_{1}=0,1, \beta_{1}=0,8$ e um

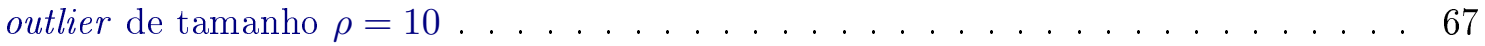

6.3 Histograma da distribuição dos valores estimados de $\alpha$ do modelo GAS(1,1) estável para simulações de $\operatorname{GARCH}(1,1)$ gaussiano com $\alpha_{0}=0,1, \alpha_{1}=0,1, \beta_{1}=0,8$ e um outlier de tamanho $\rho=10 \ldots \ldots \ldots \ldots \ldots \ldots$

6.4 Retornos diários das ações da Amazon de 15 de maio de 1997 a 5 de dezembro de

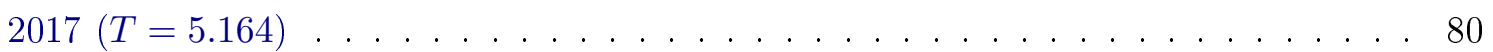

6.5 Função de autocorrelação amostral da série de retornos diários da Amazon . . . . . . 80

6.6 Função de codiferença amostral normalizada do quadrado da série de retornos diários da Amazon . . . . . . . . . . . . . . . . . . . . . . . . . 81 
6.7 Gráficos resultantes do ajuste do modelo $\operatorname{GARCH}(1,1)$ gaussiano para a série de retornos diários da Amazon . . . . . . . . . . . . . . . . . . . 84

6.8 Gráficos resultantes do ajuste do modelo $\operatorname{GARCH}(1,1)$ t-Student para a série de retornos diários da Amazon . . . . . . . . . . . . . . . . . . . 84

6.9 Gráficos resultantes do ajuste do modelo GARCH(1,1) estável para a série de retornos diários da Amazon . . . . . . . . . . . . . . . . . . . 84 84

6.10 Gráficos resultantes do ajuste do modelo $\operatorname{GAS}(1,1)$ estável para a série de retornos diários da Amazon . . . . . . . . . . . . . . . . . . 85

6.11 Função de codiferença normalizada dos resíduos do modelo GAS(1,1) estável . . . . . 85

A.1 Gráfico de $\alpha$ por $\mathcal{I}^{s t d}$ para $1<\alpha \leq 2 \ldots \ldots \ldots \ldots \ldots$ 


\section{Lista de Tabelas}

4.1 Aproximações para a função densidade em casos extremos, $1,01<\alpha \leq 2 \ldots$. . . . 31

4.2 Aproximações para $f^{* \prime}(x ; \alpha)$ em casos extremos, $1,01<\alpha \leq 2 \ldots \ldots$. . . . . . . 32

4.3 Aproximações para $f^{* \prime \prime}(x ; \alpha)$ em casos extremos, $1,01<\alpha \leq 2 \ldots \ldots 3$

4.4 Aproximações para $f^{* \prime \prime \prime}(x ; \alpha)$ em casos extremos, $1,01<\alpha \leq 2 \ldots \ldots 3$

4.5 Aproximações para $f_{\alpha}^{*}(x ; \alpha)$ nos casos extremos, $1,01<\alpha \leq 2 \ldots \ldots$. . . . . . . 34

4.6 Aproximações para $f_{\alpha \alpha}^{*}(x ; \alpha)$ nos casos extremos, $1,01<\alpha \leq 2 \ldots \ldots \ldots$

4.7 Aproximações para $f_{\alpha x}^{*}(x ; \alpha)$ nos casos extremos, $1,01<\alpha \leq 2 \ldots \ldots$

6.1 Estatísticas das distribuições dos AICs corrigidos e dos BICs dos modelos ajustados para simulações de $\operatorname{GARCH}(1,1)$ gaussiano com $\alpha_{0}=0,1, \alpha_{1}=0,1, \beta_{1}=0,8$ e um outlier de tamanho $\rho=10 \ldots \ldots \ldots \ldots \ldots 6 \ldots$

6.2 Estatísticas das distribuições dos $p$-valores dos testes AD e CVM para os resíduos dos modelos ajustados para simulações de $\operatorname{GARCH}(1,1)$ gaussiano com $\alpha_{0}=0,1$, $\alpha_{1}=0,1, \beta_{1}=0,8$ e um outlier de tamanho $\rho=10 \ldots \ldots \ldots \ldots$

6.3 Estatísticas das distribições dos MAEs para previsões da volatilidade para simulações de $\operatorname{GARCH}(1,1)$ gaussiano com $\alpha_{0}=0,1, \alpha_{1}=0,1, \beta_{1}=0,8$ e um outlier de

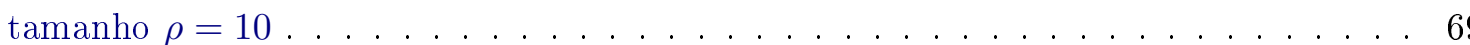

6.4 Medianas das estatísticas de ajustes para séries simuladas por processos $\operatorname{GARCH}(1,1)$ gaussianos com $\alpha_{0}=0,1, \alpha_{1}=0,1, \beta_{1}=0,8$ e outliers de tamanho $\rho=5 \ldots 70$

6.5 Medianas das estatísticas de ajustes para séries simuladas por processos $\operatorname{GARCH}(1,1)$ gaussianos com $\alpha_{0}=0,1, \alpha_{1}=0,1, \beta_{1}=0,8$ e outliers de tamanho $\rho=10 \ldots 71$

6.6 Medianas das estatísticas de ajustes para séries simuladas por processos $\operatorname{GARCH}(1,1)$ gaussianos com $\alpha_{0}=0,1, \alpha_{1}=0,1, \beta_{1}=0,8$ e outliers de tamanho $\rho=15 \ldots 72$

6.7 Medianas das estatísticas de ajustes para séries simuladas por processos GARCH $(1,1)$ gaussianos com $\alpha_{0}=0,3, \alpha_{1}=0,15, \beta_{1}=0,55$ e outliers de tamanho $\rho=5 \ldots 73$

6.8 Medianas das estatísticas de ajustes para séries simuladas por processos $\operatorname{GARCH}(1,1)$ gaussianos com $\alpha_{0}=0,3, \alpha_{1}=0,15, \beta_{1}=0,55$ e outliers de tamanho $\rho=10 \ldots 74$

6.9 Medianas das estatísticas de ajustes para séries simuladas por processos $\operatorname{GARCH}(1,1)$ gaussianos com $\alpha_{0}=0,3, \alpha_{1}=0,15, \beta_{1}=0,55$ e outliers de tamanho $\rho=15 \ldots 75$

6.10 Medianas das estatísticas de ajustes para séries simuladas por processos $\operatorname{GARCH}(1,1)$ gaussianos com $\alpha_{0}=0,5, \alpha_{1}=0,2, \beta_{1}=0,3$ e outliers de tamanho $\rho=5 \ldots \ldots$

6.11 Medianas das estatísticas de ajustes para séries simuladas por processos GARCH $(1,1)$ gaussianos com $\alpha_{0}=0,5, \alpha_{1}=0,2, \beta_{1}=0,3$ e outliers de tamanho $\rho=10 \ldots 77$ 
6.12 Medianas das estatísticas de ajustes para séries simuladas por processos $\operatorname{GARCH}(1,1)$ gaussianos com $\alpha_{0}=0,5, \alpha_{1}=0,2, \beta_{1}=0,3$ e outliers de tamanho $\rho=15 \quad$. . . 78

6.13 Estatísticas descritivas: série de retornos diários da Amazon . . . . . . . . . . . 81

6.14 Estimativas dos parâmetros dos modelos ajustados para a série de retornos diários da Amazon ................................ 82

6.15 Estatísticas de ajustes dos modelos ajustados para a série de retornos diários da Amazon ..............................8 83

A.1 Valores de $\mathcal{I}^{s t d}$ para diferentes valores de $\alpha$. . . . . . . . . . . . . . . . . . . . . 95 


\section{Capítulo 1}

\section{Introdução}

Modelos dinâmicos para séries temporais são estudados há décadas. Na prática, muitos conjuntos de dados apresentam comportamento não constante ao longo do tempo devido a vários fatores, como influência de variáveis exógenas, mudanças de regime, etc. Modelos de regressão dinâmica, de espaço de estado e bayesianos, entre outros, têm sido desenvolvidos para tais análises (Harvey (1989), West e Harrison (1997)).

Em se tratando de dados financeiros, usualmente trabalha-se com o chamado log-retorno, ou simplesmente retorno da série. Em finanças, comumente um dos objetivos é a avaliação de riscos de uma carteira de ativos (instrumentos) financeiros. O risco pode ser interpretado como a possibilidade de que uma carteira de investimentos sofra perdas em seu valor. O cálculo do risco, portanto, é a tentativa de se medir o grau de incerteza na obtenção do retorno esperado em uma determinada aplicação financeira ou investimento (Constantinides et al., 2003). É preciso, portanto, mensurar o grau de incerteza, que é frequentemente medido em termos de variações de preços dos ativos, as quais podem ser expressos pelos retornos.

Uma característica presente em tais tipos de séries de retornos é o que se convencionou chamar de volatilidade, que não é observável, associada à variabilidade de um retorno num dado instante de tempo, que pode depender de retornos passados e de outras informações disponíveis até aquele instante. Em geral, como medida de volatilidade, define-se uma variância condicional que, não sendo constante, não coincide com a variância global da série observada (Morettin, 2011).

Como nesses casos a variância condicional não é constante e depende de outras informações ao longo do tempo, pode-se enxergá-la como um parâmetro que varia no tempo, tipo $\sigma_{t}$. Os modelos ARCH (do inglês Autoregressive Conditional Heteroscedasticity) e GARCH (Generalized ARCH) são dois dos mais conhecidos para análise desse tipo de comportamento.

Além disso, um dos aspectos particulares de séries de retornos é que estas, em geral, apresentam distribuições com caudas mais pesadas do que as de uma distribuição normal, com comportamento leptocúrtico. Usualmente, analisam-se séries de retornos através de modelos ARMA-GARCH com distribuição normal ou $t$-Student. No entanto, apesar da $t$-Student conseguir se ajustar mais adequadamente a caudas pesadas, seu comportamento pode não ser suficientemente leptocúrtico, e o ajuste pode não ser satisfatório em alguns casos. Também é preciso observar que as caudas pesadas de retornos financeiros podem ocasionar a não existência de segundo momento incondicional. Nesse caso, a variância como medida de escala modelada pelos modelos GARCH com distribuição normal ou $t$-Student pode não ser apropriada (Harvey, 2013).

Os modelos GAS (Generalized Autoregressive Score), também conhecidos como DCS (Dynamic Conditional Score) foram propostos em 2008 (Creal et al. (2008), Harvey e Chakravarty (2008)), e representam um caso particular de modelos dinâmicos, sendo uma generalização de vários modelos já conhecidos na literatura.

O caráter dinâmico se dá pela evolução dos parâmetros da distribuição dos dados ao longo do tempo, como ocorre com a variância condicional em modelos GARCH. O fato dos modelos GAS incorporarem a estrutura da função score (derivada do logaritmo da verossimilhança) representa uma espécie de evolução natural dos parâmetros, baseado na distribuição dos dados (Creal et al., 
2013).

A estrutura apresenta vantagens, como facilidade na avaliação da verossimilhança e incorporação de outras complexidades, como assimetria, memória longa, comportamentos não-lineares etc. Para modelagem de séries de retornos e consequente avaliação da variância condicional, tais modelos englobam como casos particulares os modelos GARCH gaussianos. Com isso, são capazes de generalizar modelos GARCH e capturar outros comportamentos das séries. Além do GARCH, outros modelos conhecidos na literatura, como o MEM (Multiplicative Error Model) e o ACD (Autoregressive Conditional Duration), também são casos particulares do GAS (Creal et al., 2013).

Por outro lado, a classe de distribuições denominada estável apresenta uma grande flexibilidade em termos de regulagem do peso das caudas. Esse fator permite bom ajuste em diversas situações, em particular a séries de retornos financeiros. A distribuição normal é membro dessa classe e, como o nome sugere, tais distribuições são estáveis sob adição, ou seja, a soma de variáveis com distribuições estáveis também tem distribuição estável (Samorodnitsky e Taqqu, 1994).

As distribuições estáveis, comparadas com a distribuição normal, podem oferecer modelos mais realísticos para retornos de ativos financeiros de diversos tipos devido aos fatores já mencionados, com vários exemplos de aplicações bem sucedidas (Mittnik et al., 2002). Sua utilização em dados financeiros teve início na década de 1960, com destaque para os trabalhos de Benoit Mandelbrot e Eugene Fama. Mandelbrot (1963) destacou que o fato das distribuições estáveis em geral não possuírem segundo momento em decorrência de suas caudas pesadas fazem com que sejam adequadas a diversas séries reais que registram observações aparentemente atípicas.

A dificuldade no tratamento de tais distribuições reside no fato que, na maioria dos casos, não existe uma forma analítica fechada para a função densidade, apesar da função característica sempre estar disponível. Isso obviamente pode acarretar em problemas de estimação, principalmente por máxima verossimilhança. Com o avanço dos métodos computacionais, procedimentos foram desenvolvidos de modo a superar essas dificuldades (Nolan, 2001).

Panorska et al. (1995) propuseram um modelo GARCH no qual a distribuição do ruído aleatório é estável, com resultados bem sucedidos. Nesta tese, uma extensão desse modelo é proposta, associando modelos GAS a distribuições estáveis, bem como a demonstração de suas condições de estacionariedade, procedimento de estimação através de refinamentos de cálculos disponíveis na literatura, estrutura de dependência e aplicação a simulações e dados reais. A associação de modelos GAS a distribuições estáveis pode representar um ganho em termos tanto na descrição dos parâmetros dinâmicos do modelo quanto na representação do comportamento dos dados. Como a evolução dos parâmetros da distribuição dos dados nos modelos GAS é ditada pelo score ponderado da função de verossimilhança, a evolução da volatilidade ao longo do tempo pode ser mais apropriadamente descrita por uma distribuição estável caso os dados realmente sigam tal comportamento. Blasques et al. (2015) demonstraram propriedades ótimas de modelos dinâmicos guiados pelo score em termos da divergência de Kullback-Leibler.

Esta tese é composta por sete Capítulos. Os Capítulos 2 e 3 trazem introduções ao modelo GAS e às distribuições estáveis, respectivamente. O Capítulo 4 detalha cálculos das funções densidade, de suas derivadas e da informação de Fisher de distribuições estáveis, os quais exigem atenção especial pelo fato de suas fórmulas não estarem disponíveis em formas analíticas fechadas. O Capítulo 5 traz o desenvolvimento do modelo proposto, como uma generalização do modelo GARCH clássico, suas condições de estacionariedade e o procedimento de estimação. No Capítulo 6, encontram-se estudos de simulação e aplicação a dados reais e, por fim, o Capítulo 7 traz uma síntese das conclusões e os próximos passos planejados para a continuação do trabalho. 


\section{Capítulo 2}

\section{Modelos GAS}

Em muitas aplicações de interesse prático, modelos que lidam com parâmetros que variam no tempo são importantes para capturar o comportamento dinâmico de séries temporais univariadas e multivariadas. Tais modelos são frequentemente utilizados em análise econométrica (Blasques et al., 2014a).

Os modelos dinâmicos que serão aqui tratados têm em sua estrutura parâmetros da distribuição de probabilidade dos dados que não são constantes ao longo do tempo. Quando forem mencionados termos como "parâmetros variáveis" ou "parâmetros variantes no tempo", são a esses parâmetros que nos referimos. Por exemplo, a variância condicional dos dados em modelos ARCH ou GARCH. Já os parâmetros do modelo são constantes, e nos referimos a estes como "parâmetros estáticos".

Cox (1981) classifica tais modelos dinâmicos em duas classes: os guiados por observações e os guiados por parâmetros.

Em um modelo guiado por observações, os valores do parâmetro que varia no tempo são obtidos como funções determinísticas de variáveis dependentes defasadas, assim como valores atuais e defasados de variáveis exógenas. Nesse contexto, os parâmetros evoluem estocasticamente ao longo do tempo, mas podem ser preditos um passo à frente dada a informação passada. A função de verossimilhança para modelos guiados por observações está disponível em forma fechada através da decomposição do erro de predição. Esse fato leva a procedimentos simples de estimação e contribuiu para a popularidade desses modelos em econometria aplicada e estatística.

Os modelos ARCH (do inglês Autoregressive Conditional Heteroscedasticity), introduzidos por Engle (1982), e sua generalização GARCH (Generalized ARCH), introduzidos por Bollerslev (1986), são modelos não-lineares apropriados para séries financeiras que apresentam variância condicional evoluindo no tempo. Após surgirem na literatura, tais modelos tornaram-se tão utilizados e com tantas aplicações práticas que Robert Engle venceu o Prêmio Nobel de Economia, em 2003, "por métodos de análise de séries temporais econômicas com volatilidade variante no tempo (ARCH)" (Nobel, 2017). São exemplos clássicos de modelos guiados por observações.

Em modelos guiados por parâmetros, os parâmetros variam ao longo do tempo como processos dinâmicos e com fontes de erro específicas. Expressões analíticas para a função de verossimilhança dificilmente estão disponíveis em forma fechada. Logo, o cálculo da verossimilhança em geral tornase mais complicado, tipicamente sendo necessário o uso de métodos de simulação (Koopman et al., 2016). Os modelos de volatilidade estocástica, propostos por Taylor (1980), são exemplos conhecidos de modelos guiados por parâmetros. Para mais detalhes, ver Morettin (2011).

Os modelos GAS (Generalized Autoregressive Score) são modelos guiados por observações cujos parâmetros da distribuição dos dados podem variar ao longo do tempo. A principal dificuldade em formular uma estrutura unificada é a escolha de uma função que conecte as observações passadas com os valores futuros dos parâmetros. Tal função idealmente deve ser aplicável a uma classe ampla de modelos não-lineares e não-gaussianos. A função score (derivada do logaritimo da verossimilhança em relação ao parâmetro dinâmico) ponderada da densidade preditiva do modelo é uma escolha efetiva para o mecanismo de evolução dos parâmetros que variam ao longo do tempo. Ao escolher o fator de ponderação apropriadamente, modelos clássicos guiados por observações como 
GARCH, ACD (Autoregressive Conditional Duration) e ACI (Autoregressive Conditional Intensity) são obtidos. A ponderação é igualmente aplicável a modelos multivariados e leva à formulação de novos modelos guiados por observações (Creal et al., 2008).

Os modelos GAS foram introduzidos em 2008 por Creal et al. (2008) e Harvey e Chakravarty (2008), de forma independente. De acordo com Harvey (2013):

"Em 2008 escrevi um artigo com um estudante, Tirthankar Chakravarty, sobre modelos EGARCH. Ao mesmo tempo, Siem Jan Koopman e seus colegas independentemente desenvolviam um conjunto de modelos baseados no score. Eles também publicaram um artigo em 2008. Como Siem Jan e eu trabalhamos como co-autores em diversos artigos de modelos para componentes não observáveis, talvez não tenha sido surpresa que tenhamos chegado à mesma ideia, apesar de por rotas diferentes."

O modelo GAS tem um grau de generalidade semelhante ao obtido por modelos de estado de espaço não-lineares e não-gaussianos e tem as vantagens de outros modelos guiados por observações. O cálculo da verossimilhança é direto. Extensões para casos assimétricos, memória longa e outras dinâmicas complicadas podem ser considerados sem a introdução de complexidades adicionais. Como o modelo GAS é baseado no score, ele explora a estrutura completa da densidade ao invés de somente média e momentos de ordens superior. É o que diferencia os modelos GAS de outros modelos guiados por observações presentes na literatura (Creal et al., 2013).

Alguns argumentos para o uso da função score em modelos dinâmicos têm sido propostos. Por exemplo, Nelson e Foster (1994) mostraram que, para estimação do verdadeiro parâmetro variante no tempo, filtros baseados no score da densidade condicional têm uma propriedade de minimizar o erro quadrático médio. Blasques et al. (2015) mostraram que somente estruturas do parâmetro variável no tempo baseadas no score reduzem a divergência local de Kullback-Leibler entre a verdadeira densidade condicional e a densidade condicional postulada pelo modelo.

De acordo com Koopman et al. (2012), quando o processo gerador dos dados é um modelo de espaço de estados, a acurácia preditiva de um modelo GAS é equivalente à de um modelo de espaço de estados corretamente especificado. Isso é verdade em particular se a densidade observacional da especificação do GAS permite caudas pesadas e superdispersão. Eles também detectaram que modelos GAS superam muitos dos modelos familiares guidados por observações presentes na literatura. Por se apoiar na estrutura da densidade na equação de evolução dos parâmetros variáveis no tempo, os modelos GAS conseguem capturar informações adicionais dos dados de uma forma que outros modelos não conseguem.

Harvey (2013) prefere o nome DCS (do inglês Dynamic Conditional Score). Ele defende que o termo "autorregressivo" de GAS se refere a uma estrutura dinâmica mais limitada do que a verdadeira. De qualquer forma, os modelos GAS e DCS são os mesmos, e neste trabalho é utilizada a primeira nomenclatura.

\subsection{Especificação do modelo}

Nesta Seção, será abordada a especificação do modelo no caso geral. Nos Capítulos seguintes será focado o caso unidimensional.

Seja $\boldsymbol{y}_{t} \in \mathbb{R}^{N}$ um vetor aleatório $N$-dimensional no instante $t$ que represente a variável dependente de interesse e que possua distribuição condicional

$$
\boldsymbol{y}_{t} \mid \boldsymbol{y}_{1: t-1} \sim f\left(\boldsymbol{y}_{t} ; \boldsymbol{\theta}_{t}\right)=f_{t},
$$

em que $\boldsymbol{y}_{1: t-1}=\left(\boldsymbol{y}_{1}^{\prime}, \ldots, \boldsymbol{y}_{t-1}^{\prime}\right)^{\prime}$ contém a informação passada de $\boldsymbol{y}_{t}$ até o instante $t-1$ e $\boldsymbol{\theta}_{t} \in \Theta \subseteq \mathbb{R}^{J}$ é um vetor de parâmetros variantes no tempo que caracteriza de forma completa $f($.) e depende apenas de $\boldsymbol{y}_{1: t-1}$ e de um conjunto de parâmetros estáticos $\boldsymbol{\xi}$, ou seja, $\boldsymbol{\theta}_{t}=\boldsymbol{\theta}\left(\boldsymbol{y}_{1: t-1}, \boldsymbol{\xi}\right)$ para todo $t$.

Tipicamente, considera-se

$$
\boldsymbol{y}_{t}=g\left(h\left(\theta_{t}(\boldsymbol{\xi})\right), \epsilon_{t, \boldsymbol{\lambda}}\right),
$$

em que $g: \mathbb{R} \times \mathbb{R} \rightarrow \mathbb{R}$ e $h: \mathbb{R} \times \mathbb{R} \rightarrow \mathbb{R}$ são funções contínuas e diferenciáveis e $\left\{\epsilon_{t, \boldsymbol{\lambda}}\right\}_{t \in \mathbb{Z}}$ é uma 
sequência de variáveis aleatórias i.i.d., que não depende de $\boldsymbol{\theta}_{t}$ para todo $t, \operatorname{com} \epsilon_{t, \boldsymbol{\lambda}}=\epsilon_{t}$ tendo como função densidade $f_{\epsilon, \boldsymbol{\lambda}}=f_{\epsilon}$. O vetor $\boldsymbol{\lambda}$ é um vetor de parâmetros estáticos de $\epsilon_{t}$ (por exemplo, no caso de $\epsilon_{t}$ ter distribuição $t$-Student, $\boldsymbol{\lambda}$ pode se referir aos graus de liberdade).

Tal estrutura cobre muitos casos de interesse empírico. Por exemplo, um modelo de volatilidade pode ser obtido com $y_{t}=\sqrt{\theta_{t}} \epsilon_{t} \operatorname{com} \theta_{t}$ denotando a variância variante no tempo de $y_{t}$ e $\epsilon_{t}$ tendo, por exemplo, distribuição normal ou t-Student.

Em um modelo GAS, o mecanismo para evolução do parâmetro $\boldsymbol{\theta}_{t}$ de $\boldsymbol{y}_{t}$ é dado pela equação autorregressiva

$$
\boldsymbol{\theta}_{t}=\boldsymbol{\omega}+\sum_{i=1}^{p} \boldsymbol{A}_{i} \boldsymbol{s}_{t-i}+\sum_{j=1}^{q} \boldsymbol{B}_{j} \boldsymbol{\theta}_{t-j}
$$

em que $\boldsymbol{\omega}, \boldsymbol{A}_{i}$ e $\boldsymbol{B}_{j}$ são matrizes de coeficientes constantes para $i=1, \ldots, p$ e $j=1, \ldots, q$ com dimensões apropriadas, cujas componentes, mais as componentes de $\boldsymbol{\lambda}$, constituem o conjunto de parâmetros estáticos do modelo $\boldsymbol{\xi}$, e $\boldsymbol{s}_{t}$ é uma função de ponderação apropriada, que depende das observações passadas, de $\boldsymbol{\theta}_{t}$ e dos parâmetros estáticos de $\boldsymbol{\xi}$. A principal contribuição do modelo é a escolha do mecanismo de evolução com base em $\boldsymbol{s}_{t}$, aplicável em uma classe ampla de densidades e modelos não-lineares.

A utilização de $\boldsymbol{s}_{t}$ na equação (2.3) é o principal fator de diferença para uma especificação típica de evolução de parâmetro dinâmico. $\boldsymbol{s}_{t}$ é um vetor proporcional ao score:

$$
\boldsymbol{s}_{t}=\boldsymbol{s}\left(\boldsymbol{y}_{t}, \boldsymbol{\theta}_{t}\right)=\boldsymbol{S}\left(\boldsymbol{\theta}_{t}\right) \boldsymbol{\nabla}\left(\boldsymbol{y}_{t}, \boldsymbol{\theta}_{t}\right)
$$

A matriz $\boldsymbol{S}\left(\boldsymbol{\theta}_{t}\right)=\boldsymbol{S}_{t}$ é uma matriz de ponderação $J \times J$ positiva definida e

$$
\boldsymbol{\nabla}\left(\boldsymbol{y}_{t}, \boldsymbol{\theta}_{t}\right)=\nabla_{t}=\frac{\partial \ln f\left(\boldsymbol{y}_{t} ; \boldsymbol{\theta}_{t}\right)}{\partial \boldsymbol{\theta}_{t}}
$$

é o score calculado em $\boldsymbol{\theta}_{t}$, em que $f\left(\boldsymbol{y}_{t} ; \boldsymbol{\theta}_{t}\right)=f_{t}$ é a função densidade de $\boldsymbol{y}_{t}$. As equações (2.3)-(2.5) constituem o modelo GAS com ordens $p$ e $q$, denotado por $\operatorname{GAS}(p, q)$ (Creal et al., 2008).

A utilização do score para a evolução de $\boldsymbol{\theta}_{t}$ é intuitiva, pois representa a direção da subida mais acentuada para melhorar o ajuste local em termos da verossimilhança no instante $t$, dada a posição de $\boldsymbol{\theta}_{t}$. A estrutura pode ser interpretada como a direção natural para a evolução do parâmetro ao longo do tempo. Além disso, o score depende da densidade completa, e não apenas de momentos de primeira ou de segunda ordens. Essa é a principal diferença da estrutura do modelo GAS para a maioria dos modelos guiados por observações presentes na literatura. Ao explorar toda a estrutura da densidade, o modelo GAS introduz novas transformações nos dados que podem ser usadas para a evolução de $\boldsymbol{\theta}_{t}$.

Através da escolha da função de ponderação $\boldsymbol{S}_{t}$, o modelo GAS permite uma flexibilidade adicional em como o score é usado para a evolução de $\boldsymbol{\theta}_{t}$. É importante notar que, para cada escolha de $\boldsymbol{S}_{t}$, tem-se um modelo GAS diferente. As propriedades estatísticas de cada um desses modelos podem ser diferentes e exigem atenção.

Creal et al. (2013) discutem escolhas apropriadas para $\boldsymbol{S}_{t}$ baseadas na curvatura da log-verossimilhança no instante $t$ sumarizadas pela matriz de informação de Fisher, dessa forma ligando o fator de ponderação à variância do score. A sugestão é fazer com que a matriz de ponderação seja uma potência $v>0$ da inversa da matriz de informação de $\boldsymbol{\theta}_{t}$ para levar em conta a variância de $\nabla_{t}$. Ou seja,

$$
\boldsymbol{S}_{t}=\mathcal{I}_{t}^{-v}
$$

com

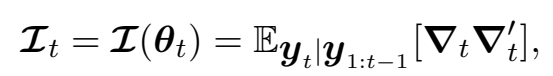

em que a esperança é tomada com respeito à distribuição condicional de $\boldsymbol{y}_{t}$ dado $\boldsymbol{y}_{1: t-1}$. Daqui para frente, para simplificar a notação, tal esperança será denotada por $\mathbb{E}_{t}$.

Se $v=1, \boldsymbol{S}_{t}$ é a inversa da matriz de informação, e a equação de evolução (2.3) para $\boldsymbol{\theta}_{t}$ se 
reduz a uma evolução semelhante a do tipo Newton-Raphson para cada nova observação $\boldsymbol{y}_{t}$ que se torna disponível ao longo do tempo. Usando essa escolha para ponderar o score, o modelo GAS pode englobar os conhecidos modelos guiados por observações GARCH, ACD e ACI.

Se $v=1 / 2, \boldsymbol{S}_{t}$ é a inversa da raiz quadrada da matriz de informação. Uma vantagem dessa escolha para $\boldsymbol{S}_{t}$ é que as propriedades estatísticas do modelo GAS correspondente tornam-se mais tratáveis, pois, neste caso, $\boldsymbol{s}_{t}$ tem variância constante e unitária, o que pode ser útil para detectar a má especificação do modelo em aplicações (Koopman et al., 2016).

Se $v=0$, o fator de ponderação é a identidade, ou seja, $\boldsymbol{S}_{t}=\boldsymbol{I}$ em (2.4). Nesse caso, obtémse modelos como o ACM (Autoregressive Conditional Multinomial) de Russell e Engle (2005). No contexto de uma densidade genérica $f\left(\boldsymbol{y}_{t} ; \boldsymbol{\theta}_{t}\right)$, no entanto, as propriedades estatísticas do modelo GAS nesse contexto são muito mais complicadas.

Para qualquer escolha de $v, s_{t}$ é uma diferença martingal com respeito à distribuição condicional de $\boldsymbol{y}_{t}$ dado $\boldsymbol{y}_{1: t-1}$, ou seja, $\mathbb{E}_{t}\left[\boldsymbol{s}_{t}\right]=\mathbf{0}$ para qualquer $t$. No modelo $\operatorname{GAS}(1,1)$, se as raízes de $\boldsymbol{I}-\boldsymbol{B}_{1}$ estão dentro do círculo unitário, ou seja, se o raio espectral de $\boldsymbol{B}_{1}$ é menor que um (se $\max \left(\left|\tau_{1}\right|, \ldots,\left|\tau_{J}\right|\right)<1$, em que $\tau_{i}$ é o $i$-ésimo autovalor de $\left.\boldsymbol{B}_{1}\right)$, tem-se, por substituições recursivas,

$$
\boldsymbol{\theta}_{t}=\sum_{i=0}^{\infty} \boldsymbol{B}_{1}^{i} \boldsymbol{\omega}+\sum_{i=1}^{\infty} \boldsymbol{B}_{1}^{i-1} \boldsymbol{A}_{1} \boldsymbol{s}_{t-i} .
$$

Como $\mathbb{E}_{t}\left[\boldsymbol{s}_{t}\right]=\mathbf{0}$ e $\sum_{i=1}^{\infty} \boldsymbol{B}_{1}^{i}=\left(\boldsymbol{I}-\boldsymbol{B}_{1}\right)^{-1}$, então, a longo prazo, o valor de $\boldsymbol{\theta}_{t}$ convergirá para $\left(\boldsymbol{I}-\boldsymbol{B}_{1}\right)^{-1} \boldsymbol{\omega}$, sendo seu valor não-condicional. Logo, o vetor $\boldsymbol{\omega}$ e a matriz $\boldsymbol{B}_{1}$ controlam o nível e a persistência do processo, respectivamente (Ardia et al., 2016).

Já a matriz $\boldsymbol{A}_{1}$ que multiplica o score ponderado $\boldsymbol{s}_{t}$ controla o impacto de $\boldsymbol{s}_{t}$ em $\boldsymbol{\theta}_{t+1}$. Como detalhado em Creal et al. (2013), a quantidade $\boldsymbol{s}_{t}$ indica a direção de evolução do vetor de parâmetros de $\boldsymbol{\theta}_{t}$ para $\boldsymbol{\theta}_{t+1}$, agindo como um algoritmo de subida mais acentuada para melhorar o ajuste local do modelo dada a posição atual do parâmetro. Dessa forma, o procedimento se assemelha ao clássico algoritmo de Newton-Raphson, em que $\boldsymbol{A}_{1}$ pode ser interpretada como o tamanho do passo de atualização, e que precisa ser ajustado de uma forma a não distorcer a informação contida em $\boldsymbol{s}_{t}$ (Ardia et al., 2016).

Pode-se também generalizar a equação de evolução (2.3) em várias direções. Por exemplo, pode ser interessante incluir variáveis exógenas em (2.3) ou generalizar a evolução de $\boldsymbol{\theta}_{t}$ incluindo outros efeitos não-lineares tais como mudanças de regine. Além disso, pode ser apropriado considerar versões de memória longa de (2.3) para obter uma especificação de modelos GAS integrados fracionários em um contexto similar aos dos conhecidos modelos ARFIMA e FIGARCH (Creal et al., 2013).

\subsection{Modelos GAS para volatilidade}

$\mathrm{O}$ risco de uma carteira de ativos financeiros se refere à possibilidade da carteira sofrer perdas em seu valor devido a algum fator. Quanto maior a possibilidade de mudança no preço do ativo, maior é o risco desse ativo. Da mesma forma, o risco de uma carteira provém de sua volatilidade. Mensurar tal risco, portanto, corresponde a encontrar métodos para mensurar a volatilidade da carteira (Figueira Neto, 2013).

A volatilidade é assumida como a variabilidade de algum fenômeno ao longo do tempo. Usualmente é obtida através do desvio padrão ou da variância dos retornos, apesar de poder ser obtida através de outras estatísticas associadas à variabilidade.

Ou seja, em geral séries de retornos financeiros possuem heteroscedasticidade condicional, apresentando a variância condicional evoluindo no tempo.

Há grande variedade de modelos não-lineares para modelagem da volatilidade de séries financeiras, sendo os das classes ARCH e GARCH alguns dos mais utilizados. Para mais detalhes acerca desses modelos, ver Morettin (2011).

Baseado na estrutura do modelo GARCH, é possível formular um modelo GAS para modelagem 
de volatilidade.

Considere um modelo GAS univariado na especificação do modelo em (2.3)-(2.5) $\operatorname{com} \boldsymbol{\theta}_{t}=\sigma_{t}$. Na especificação de $y_{t}$ em $(2.2)$, considere que $g\left(h\left(\sigma_{t}\right), \epsilon_{t}\right)=\sqrt{\sigma_{t}} \epsilon_{t} . \sigma_{t}$ é a variância de $y_{t}$ variante no tempo. Suponha que $\sigma_{t}$ seja determinado pela equação do modelo GAS

$$
\sigma_{t}=\omega+\sum_{i=1}^{p} A_{i} s_{t-i}+\sum_{j=1}^{q} B_{j} \sigma_{t-j}
$$

em que $s_{t}$ é o score ponderado. Tal estrutura é um modelo para volatilidade, afinal determina como $\sigma_{t}$, a variância condicional da série $y_{t}$, evolui ao longo do tempo.

Pode-se verificar que o modelo GARCH com distribuição condicional normal é um caso particular de tal modelo GAS.

Considere $\epsilon_{t} \sim N(0,1)$. Logo, $y_{t}=\sqrt{\sigma_{t}} \epsilon_{t} \sim N\left(0, \sigma_{t}\right)$, e $f\left(y_{t} ; \sigma_{t}\right)$ é a função densidade dessa distribuição. Pode-se verificar que

$$
\begin{aligned}
\nabla_{t} & =\frac{\partial \ln f\left(y_{t} ; \sigma_{t}\right)}{\partial \sigma_{t}}=-\frac{1}{2 \sigma_{t}}+\frac{y_{t}^{2}}{2 \sigma_{t}^{2}}, \\
\frac{\partial \nabla_{t}}{\partial \sigma_{t}} & =\frac{\partial^{2} \ln f\left(y_{t} ; \sigma_{t}\right)}{\partial \sigma_{t}^{2}}=\frac{1}{2 \sigma_{t}^{2}}-\frac{y_{t}^{2}}{\sigma_{t}^{3}} .
\end{aligned}
$$

Considere também que a escolha para o fator de ponderação do score seja $S_{t}=\mathcal{I}_{t}^{-1}$. Logo,

$$
s_{t}=S_{t} \nabla_{t}=-\mathbb{E}_{t}\left[\frac{\partial^{2} \ln f\left(y_{t} ; \sigma_{t}\right)}{\partial \sigma_{t}^{2}}\right]^{-1} \nabla_{t}=2 \sigma_{t}^{2}\left(-\frac{1}{2 \sigma_{t}}+\frac{y_{t}^{2}}{2 \sigma_{t}^{2}}\right)=y_{t}^{2}-\sigma_{t} .
$$

Substituindo em (2.9),

$$
\sigma_{t}=\omega+\sum_{i=1}^{p} A_{i}\left(y_{t-i}^{2}-\sigma_{t-i}\right)+\sum_{j=1}^{q} B_{j} \sigma_{t-j}=\omega+\sum_{i=1}^{p} A_{i} y_{t-i}^{2}+\sum_{j=1}^{q}\left(B_{j}-A_{j}\right) \sigma_{t-j},
$$

em que $A_{j}=0$ para $j=p+1, \ldots, q$. O modelo é equivalente ao modelo $\operatorname{GARCH}(p, q)$ padrão gaussiano, introduzido por Bollerslev (1986) e um dos mais utilizados para volatilidade condicional.

Pode-se perceber que $p>q$ não é uma condição permitida nesta representação, pois, de acordo com as condições de estacionariedade e para que $\sigma_{t}$ se mantenha positivo, as condições $A_{i} \geq 0$, $i=1, \ldots, p$, e $B_{j}-A_{j} \geq 0, j=1, \ldots, q$ devem ser obedecidas (Bollerslev, 1986). No caso de $p>q$, necessariamente $B_{j}-A_{j} \leq 0$ para $j=q+1, \ldots, p$. De qualquer forma, o modelo GARCH com $p=1$ e $q=1$, cuja ordem é a mais utilizada na prática, é permitido nessa representação e é um caso particular de modelo GAS.

Os modelos GARCH são amplamente utilizados para modelar a variância condicional de séries temporais. No entanto, a utilização da normal como distribuição condicional pode não ser a mais adequada, pois um fato usual em retornos financeiros é que eles são caracterizados por caudas pesadas. Bollerslev (1987) propôs um modelo GARCH com distribuição condicional $t$-Student como alternativa à estrutura gaussiana. Tal modelo é amplamente utilizado na prática e também como um ponto de partida para outros modelos (Harvey, 2013).

Por outro lado, os modelos GAS têm se revelado muito úteis na modelagem de dados de caudas pesadas ou assimétricos, como são muito encontrados em finanças. As dinâmicas das correlações e volatilidades desses modelos são guiadas pelo score da distribuição condicional preditiva. Se a distribuição tem cauda pesada, as dinâmicas guiadas pelo score automaticamente são corrigidas por observações extremas. Dessa forma, tais modelos têm semelhanças com modelos GARCH robustos. A abordagem pelo score, no entanto, oferece uma estrutura mais geral e unificada para a dinâmica dos parâmetros que pode ser aplicada além do contexto de volatilidade e correlação (Blasques et al., 2016)

Creal et al. (2011) e Harvey e Luati (2014) utilizaram modelos GAS com distribuições condici- 
onais $t$-Student para modelagem de volatilidade. Tais modelos adequam outliers de uma maneira natural quando atualizam as correlações e volatilidades ao longo do tempo.

Quando a distribuição $t$-Student é empregada na distribuição preditiva dos retornos na modelagem GARCH, a formulação da equação da variância condicional não é alterada e mantém-se a mesma do modelo gaussiano. A especificação da variância condicional é uma combinação linear de observações defasadas ao quadrado, e a consequência é que é muito sensível a observações extremas. Dessa forma, quanto estas surgem, seu efeito se dissipa muito lentamente. Se a equação dinâmica para a volatilidade depender do score ao invés das observações defasadas ao quadrado, o modelo resultante diluirá o efeito dos outliers (Harvey, 2013). Tal modelo se transformará no clássico GARCH gaussiano quando os graus de liberdade tendem a infinito.

Conforme verificado, o clássico GARCH gaussiano é um caso particular de um modelo GAS. No entanto, não existe a mesma relação entre o modelo GARCH com distribuição condicional $t$ Student e o modelo GAS. Se assumirmos que $\epsilon_{t}$ segue uma distribuição $t$-Student com $\nu$ graus de liberdade e variância unitária, a especificação $\operatorname{GAS}(1,1)$ para a variância condicional resulta em uma equação de evolução para $\sigma_{t}$ diferente da do modelo GARCH com distribuição $t$-Student. A estrutura da equação ocasiona um aumento mais moderado na variância para um valor alto de $\left|y_{t}\right|$, quando o número de graus de liberdade é finito. Lembre-se que, no modelo GARCH, a variância condicional é afetada pelo valor quadrático de $y_{t}$. No modelo GAS, se os erros são modelados por uma distribuição de cauda pesada, uma observação $y_{t}$ com valor absoluto alto não necessita de um aumento substancial na variância. Logo, o mecanismo de evolução do GAS para o modelo com erros $t$-Student é notavelmente diferente de seu correspondente GARCH. Em outras palavras, a utilização utilizando o score implica em um modelo com equação de evolução mais robusta da variância ao longo do tempo (Blasques et al., 2015). A estrutura deste modelo específico e suas vantagens na prática serão detalhadas na Subseção 5.1.3.

A estrutura do modelo GAS também permite que outras distribuições de caudas pesadas sejam utilizadas. Creal et al. (2013) considera a distribuição de Laplace assimétrica e Harvey (2013) utiliza a GED (distribuição generalizada de erros).

Uma distribuição estável como distribuição de caudas pesadas também pode ser considerada, e em alguns casos pode ser mais adequada do que a distribuição $t$-Student quando os dados têm distribuição leptocúrtica. Tal combinação ainda não foi considerada na literatura. Esse é o principal propósito deste trabalho. Uma revisão de distribuições estáveis será feita no Capítulo 3, e o modelo proposto será apresentado no Capítulo 5.

\subsection{Justificativa teórica para o uso do score}

Blasques et al. (2015) obtiveram uma justificativa teórica para o uso do score para evolução do parâmetro da distribuição de probabilidae dos dados que varia no tempo em modelos guiados por observações. A justificativa formal é baseada na divergência de Kullback-Leibler. Os autores demonstram que utilização do score reduz a divergência de Kullback-Leibler entre a verdadeira densidade condicional dos dados $f_{t}=f\left(y_{t} ; \theta_{t}\right)$ e a densidade condicional $\tilde{f}_{t}=\tilde{f}\left(y_{t} ; \tilde{\theta}_{t}\right)$ obtida pelo modelo. Em particular, a evolução baseada no score reduz a divergência de Kullback-Leibler em valor esperado a cada passo. Além disso, apenas estruturas baseadas no score têm essa propriedade.

A distância (divergência) de Kullback-Leibler foi introduzida por Kullback e Leibler (1951) como uma medida de como uma distribuição de probabilidade diverge de outra. Hobson (1971) demonstrou que a divergência de Kullback-Leibler é a única medida de informação que satisfaz um conjunto de propriedades desejáveis. Dada sua fundamental importância em teoria de informação, aprendizagem de máquinas, econometria, mecânica estatística e muitos outros campos, a divergência de Kullback-Leibler pode ser vista como um ponto de partida natural para desenvolver uma motivação teórica para o uso da função score em modelos guiados por observações (Blasques et al., 2015).

Seja $\left\{\theta_{t}\right\}_{t \in \mathbb{Z}}$ uma sequência verdadeira e não observável de parâmetros que evoluem no tempo de uma distribuição $f\left(. \mid \theta_{t}\right)$, e uma sequência filtrada $\left\{\tilde{\theta}_{t}\right\}=\left\{\tilde{\theta}\left(y_{1: t-1} ; \boldsymbol{\xi}\right)\right\}$ tipicamente obtida por um modelo, em que $\boldsymbol{\xi}$ é um vetor de parâmetros estáticos. A atualização ótima de $\tilde{\theta}_{t}$ para o valor 
seguinte $\tilde{\theta}_{t+1}$ minimiza a divergência de Kullback-Leibler entre a verdadeira densidade condicional $f_{t}=f\left(. \mid \theta_{t}\right)$ e a densidade postulada $\tilde{f}_{t+1}=\tilde{f}\left(. \mid \tilde{\theta}_{t+1} ; \boldsymbol{\xi}\right)$. A divergência de Kullback-Leibler é definida por

$$
\mathcal{D}\left(f_{t}, \tilde{f}_{t+1}\right)=\int_{Y} f\left(y \mid \theta_{t}\right) \ln \frac{f\left(y \mid \theta_{t}\right)}{\tilde{f}\left(y \mid \tilde{\theta}_{t+1} ; \boldsymbol{\xi}\right)} d y,
$$

em que $Y \subseteq \mathbb{R}$ é o subconjunto no qual a divergência é calculada.

Em um modelo guiado por obervações, o objetivo é aproximar $f_{t}$ por $\tilde{f}\left(. \mid \tilde{\theta}_{t+1}\right)$, já que a atualização de $\tilde{\theta}_{t}$ para $\tilde{\theta}_{t+1}$ é baseada em observar uma realização $y_{t}$ de $f_{t}$. Blasques et al. (2015) argumentam que, à primeira vista, pode parecer mais apropriado aproximar $f_{t+1} \operatorname{por} \tilde{f}\left(. \mid \tilde{\theta}_{t+1}\right)$, mas não faz sentido pensar que $\tilde{f}\left(. \mid \tilde{\theta}_{t+1}\right)$ aproxime $f_{t+1}$ utilizando uma realização $y_{t}$ de $f_{t}$.

A melhoria que o processo produz em termos da divergência de Kullback-Leibler em relação à densidade condicional verdadeira é uma noção importante no contexto aqui abordado. Em particular, dado um ponto de partida para a sequência filtrada $\tilde{\theta}_{t}$ e uma densidade condicional $\tilde{f}\left(. \mid \tilde{\theta}_{t}\right)$, analisam-se as condições sob as quais a nova observação $y_{t}$, observada de $f\left(. \mid \theta_{t}\right)$, produz uma atualização de $\tilde{\theta}_{t}$ para $\tilde{\theta}_{t+1}$ tal que a nova densidade condicional $\tilde{f}\left(. \mid \tilde{\theta}_{t+1}\right)$ fornece uma melhor aproximação para $f\left(. \mid \theta_{t}\right)$ do que $\tilde{f}\left(. \mid \tilde{\theta}_{t}\right)$. A mudança na divergência de Kullback-Leibler que resulta da atualização de um ponto $\tilde{\theta}_{t}$ para outro ponto $\tilde{\theta}_{t+1}$ é chamada de variação realizada de Kullback-Leibler.

Definição 2.3.1 A variação realizada de Kullback-Leibler da atualização de $\tilde{\theta}_{t}$ para $\tilde{\theta}_{t+1}$ é definida por

$$
\Delta_{t \mid t}=\mathcal{D}\left(f_{t}, \tilde{f}_{t+1}\right)-\mathcal{D}\left(f_{t}, \tilde{f}_{t}\right)=\int_{Y} f\left(y \mid \theta_{t}\right)\left\{\ln \tilde{f}\left(y \mid \tilde{\theta}_{t} ; \boldsymbol{\xi}\right)-\ln \tilde{f}\left(y \mid \tilde{\theta}_{t+1}\right)\right\} d y .
$$

Para um dado $\theta_{t}$, o mecanismo de atualização é dito ótimo em termos da variação realizada de Kullback-Leibler se e somente se $\Delta_{t \mid t}<0$.

Em um sistema dinâmico sujeito a perturbações estocásticas, nem sempre é possível ter certeza que a atualização realizada é ótima. Com atualizações baseadas no score não é diferente. No entanto, um procedimento ótimo de atualização deve ter a tendência de se mover, em média, na direção correta, no sentido de que a divergência de Kullback-Leibler diminua em valor esperado. Tendo isso em vista, uma ferramenta importante é o conceito da variação condicional de Kullback-Leibler.

Definição 2.3.2 A variação condicional de Kullback-Leibler da atualização de $\tilde{\theta}_{t}$ para $\tilde{\theta}_{t+1}$ é definida por

$$
\Delta_{t \mid t-1}=\int_{F} q\left(\tilde{\theta}_{t+1} \mid \tilde{\theta}_{t}, \theta_{t} ; \boldsymbol{\xi}\right)\left\{\int_{Y} f\left(y \mid \theta_{t}\right) \ln \frac{f\left(y \mid \theta_{t}\right)}{\tilde{f}\left(y \mid \tilde{\theta}_{t}\right)} d y\right\} d \tilde{\theta}_{t+1}
$$

em que $F \subseteq \mathbb{R}$ é o subconjunto da reta real no qual se encontra $\tilde{\theta}_{t}$ e $q\left(\tilde{\theta}_{t+1} \mid \tilde{\theta}_{t}, \theta_{t} ; \boldsymbol{\xi}\right)$ denota a densidade de $\tilde{\theta}_{t+1}$ condicionada por $\tilde{\theta}_{t}$ e $\theta_{t}$. Para uma dada densidade $f_{t}$, uma atualização é ótima em termos da variação condicional de Kulback-Leibler se e somente se $\Delta_{t \mid t-1}<0$ para todo $\left(\tilde{\theta}_{t}, \theta_{t}\right)$.

Digamos que o mecanismo de atualização de $\tilde{\theta}_{t+1}$ através de um modelo baseado no score seja dado por

$$
\tilde{\theta}_{t+1}=\omega+A_{1} S_{t} \tilde{\nabla}_{t}+B_{1} \tilde{\theta}_{t}
$$

para um esquema de primeira ordem autorregressivo, em que $\tilde{\nabla}_{t}$ é a função score avaliada em $\tilde{\theta}_{t}$ e $S_{t}=S\left(\tilde{\theta}_{t}\right)$ é uma função positiva de ponderação que pode depender de $\tilde{\theta}_{t}$ e do conjunto de parâmetros estáticos $\boldsymbol{\xi}$, que é composto por $\omega, A_{1}$ e $B_{1}$.

Assuma que a densidade condicional $f(y \mid \theta)$ seja estritamente positiva para todo $(y, \theta) \in \mathbb{R} \times \Theta$, e $\tilde{\nabla}(\tilde{\theta}, y ; \boldsymbol{\xi}) \neq 0$ para todo $(\tilde{\theta}, \boldsymbol{\xi}) \in \tilde{\Theta} \times \Xi$ e todo $y \in \mathbb{R}$. A primeira condição exclui os valores de $\theta$ que resultam em uma distribuição degenerada para $y$, e a segunda condição exclui a possibilidade de que $\theta$ seja não-identificado em certos passos durante sua evolução.

Também assuma que o parâmetro $A_{1}$ e a quantidade de ponderação $S(\tilde{\theta} ; \boldsymbol{\xi})$ sejam estritamente positivos para todo $(\tilde{\theta}, \boldsymbol{\xi}) \in \tilde{\Theta} \times \Xi$. Essa condição garante que a informação contida no score não seja ignorada ou distorcida. 
Defina a atualização de Newton-score como (2.17) com $\omega=0$ e $B_{1}=1$. O esquema se assemelha com a atualização do clássico método de Newton.

Proposição 2.3.1 Sob tais condições, toda atualização de Newton-score é localmente ótima em termos da variação realizada de Kullback-Leibler e localmente ótima em termos da variação condicional de Kullback-Leibler para qualquer densidade verdadeira $f_{t}$.

Em outras palavras, a proposição mostra que as restrições $A_{1}>0$ e $\left(\omega, B_{1}\right)=(0,1)$ garantem que existem $\delta_{\theta}>0$ e $\delta_{y}>0$ tais que $\Delta_{t \mid t-1}<0$ e $\Delta_{t \mid t}<0$ para todo $f_{t}$ em uma vizinhança de $\tilde{\theta}_{t}$ e $y_{t}$ em conjuntos da forma

$$
\begin{aligned}
& F=F_{\delta_{\theta}}\left(\tilde{\theta}_{t}\right)=\left\{\tilde{\theta} \in \tilde{\Theta}:\left|\tilde{\theta}-\tilde{\theta}_{t}\right|<\delta_{\theta}\right\}, \\
& Y=Y_{\delta_{y}}\left(y_{t}\right)=\left\{y \in \mathcal{Y}:\left|y-y_{t}\right|<\delta_{y}\right\} .
\end{aligned}
$$

Tal resultado é alcançado pois, localmente, o sinal do score indica corretamente se o parâmetro variante no tempo deve ser atualizado positivamente ou negativamente.

Essas propriedades podem ser generalizadas para atualizações com outros valores de $\omega$ e $B_{1}$, mas algumas condições são necessárias. Blasques et al. (2015) fornecem condições específicas para garantir que o mecanismo de atualização $\tilde{\theta}_{t+1}=\omega+A_{1} S_{t} \tilde{\nabla}_{t}+B_{1} \tilde{\theta}_{t}$ do modelo GAS seja localmente ótimo em termos da variação realizada de Kullback-Leibler e localmente ótimo em termos da variação condicional de Kullback-Leibler.

Proposição 2.3.2 A atualização pelo score é localmente ótima em termos da variação realizada de Kullback-Leibler para todo $f_{t}$ se

$$
A_{1}>\frac{\left|\omega+\left(B_{1}-1\right) \tilde{\theta}_{t}\right|}{S\left(\theta_{t} ; \boldsymbol{\xi}\right)\left|\tilde{\nabla}\left(\tilde{\theta}_{t}, y_{t} ; \boldsymbol{\xi}\right)\right|}
$$

quase certamente para todo t. A atualização pelo score é localmente ótima em termos da variação condicional de Kullback-Leibler para todo $f_{t}$ se

$$
A_{1}>\frac{\mathbb{E}_{Y_{\theta}}^{t-1}\left[\left|\tilde{\nabla}\left(\tilde{\theta}_{t}, y_{t} ; \boldsymbol{\xi}\right)\right|\right]}{S\left(\theta_{t} ; \boldsymbol{\xi}\right) \mathbb{E}_{Y_{\theta}}^{t-1}\left[\left|\tilde{\nabla}\left(\tilde{\theta}_{t}, y_{t} ; \boldsymbol{\xi}\right)\right|^{2}\right]}\left|\omega+\left(B_{1}-1\right) \tilde{\theta}_{t}\right|
$$

quase certamente para todo $t$, em que

$$
\mathbb{E}_{Y_{\theta}}^{t-1}\left[\left|\tilde{\nabla}\left(\tilde{\theta}_{t}, y_{t} ; \boldsymbol{\xi}\right)\right|\right]=\int_{Y_{\theta}} f\left(y_{t} \mid \theta_{t}\right)\left|\tilde{\nabla}\left(\tilde{\theta}_{t}, y_{t} ; \boldsymbol{\xi}\right)\right| d y
$$

com

$$
Y_{\theta}=\left\{y \in \mathbb{R}^{N}:\left|\omega+A_{1} S_{t} \tilde{\nabla}_{t}+B_{1} \tilde{\theta}_{t}-\tilde{\theta}_{t}\right|<\delta_{\theta}\right\}
$$

Exemplo 2.3.1 Foi verificado anteriormente que um caso particular do modelo GAS, em que a distribuição normal é considerada, é o modelo GARCH (ver relação (2.13)). Nesse caso, a condição necessária expressa em (2.20) se torna $A_{1}\left|y_{t}^{2}-\tilde{\theta}_{t}\right|>\left|\omega+\left(B_{1}-1\right) \tilde{\theta}_{t}\right|$.

Blasques et al. (2015) também estendem os resultados locais para um conjunto global.

As regiões ótimas são válidas para qualquer densidade condicional postulada $\tilde{f}$, independente do verdadeiro e desconhecido processo gerador dos dados $f$. Ao mesmo tempo, no entanto, nem todas as escolhas de densidade $\tilde{f}$ são as mesmas. Para minimizar a divergência de Kullback-Leibler, é sempre preferível trabalhar com um modelo de densidade que aproxime da melhor forma possível o processo gerador dos dados. Dessa forma, uma boa escolha para a densidade condicional é crucial. Os resultados garantem apenas que, para uma dada escolha de densidade, a evolução de $\tilde{\theta}$ guiada pelo score de $\tilde{f}$ possui propriedades ótimas. Atualizações guiadas por observações baseadas em outros mecanismos não necessariamente possuem as mesmas propriedades. 


\subsection{Estacionariedade}

Apesar da eficácia observada pelos modelos GAS e de sua capacidade de generalização, relativamente pouco se conhecia até bem pouco tempo atrás sobre o comportamento estocástico geral de processos GAS à exceção de alguns casos particulares bem conhecidos tais como os modelos GARCH gaussiano e Beta-t-GARCH (Harvey, 2013). Blasques et al. (2014a) trataram desse assunto ao fornecerem condições explícitas para estacionariedade e ergodicidade de uma classe de processos GAS. Em particular, caracterizaram a região do espaço paramétrico que fazem do processo estacionário e ergódico. O estabelecimento de estacionariedade e ergodicidade não apenas permite um entendimento melhor da natureza do processo GAS como também é essencial para demonstrações de consistência e normalidade assintótica dos estimadores.

O fato da estrutura do modelo GAS ser tão geral e permitir diferentes aspectos como distribuição dos dados e seleção da função de ponderação é um fator de complicação. Cada escolha leva a um modelo diferente e reflete diretamente nas propriedades de estacionariedade e ergodicidade do parâmetro variante no tempo.

Blasques et al. (2014a) demonstraram as regiões de estacionariedade e ergodicidade para o modelo GAS(1,1) univariado. A não-linearidade das recursões do modelo GAS permite apenas estabelecer condições suficientes.

Seja o modelo definido por

$$
\begin{aligned}
y_{t} & =g\left(h\left(\theta_{t}(\boldsymbol{\xi})\right), \epsilon_{t}\right), \\
\theta_{t} & =\omega+A_{1} s_{t-1} B_{1} \theta_{t-1}, \\
s_{t} & =S_{t} \nabla_{t} .
\end{aligned}
$$

As propriedades estocásticas de $y_{t}$ são completamente determinadas por: (i) a parametrização $h$; (ii) a família de densidades $f_{\epsilon}=\left\{f_{\epsilon, \boldsymbol{\lambda}}\right\}_{\boldsymbol{\lambda} \in \boldsymbol{\Lambda}}$; (iii) a família de funções de transformação $g$; (iv) a função de ponderação $S_{t}$; e (v) o valor de $\boldsymbol{\xi}$, que engloba os parâmetros estáticos $\boldsymbol{\lambda}, \omega, A_{1}$ e $B_{1}$.

Para demonstração de estacionariedade, algumas suposições são necessárias, como por exemplo a função $g$ ser contínua. Duas delas merecem destaque:

Suposição 2.4.1 A função $s_{t}=s\left(\theta_{t}\right)$ deve poder ser escrita como $s_{t}\left(\theta_{t}\right)=\zeta_{1, t} \theta_{t}+\zeta_{2, t}$, em que $\zeta_{1, t}$ $e \zeta_{2, t}$ são variáveis aleatórias reais.

Suposição 2.4.2 $s_{t}$ deve ser continuamente diferenciável com

$$
\left|\frac{\partial s\left(\theta_{t}\right)}{\partial \theta_{t}}\right| \leq\left|\eta\left(\theta_{t}\right) \zeta_{1}\left(\epsilon_{t}\right)+\zeta_{2}\left(\epsilon_{t}\right)\right| \quad \forall \theta_{t} \in \Theta
$$

em que $\sup _{\theta_{t} \in \Theta}\left|\eta\left(\theta_{t}\right)\right| \leq \bar{\eta}<\infty e \mathbb{E}\left(\left|\zeta_{i}\left(\epsilon_{t}\right)\right|\right) \leq \bar{\zeta}_{i}<\infty, i=1,2$.

Proposição 2.4.1 Se st é quase certamente continuamente diferenciável em $\theta_{t}$, então uma condição suficiente para que $\theta_{t}$ convirja exponencialmente para uma sequência estacionária e ergódica é dada por

$$
\mathbb{E}\left(\sup _{\theta_{t}^{*} \in \Theta}\left|B_{1}+A_{1} \frac{\partial s\left(\theta_{t}^{*}\right)}{\partial \theta_{t}}\right|\right)<1,
$$

o que é implicado por

$$
\mathbb{E}\left(\sup _{\theta_{t}^{*} \in \Theta}\left|\frac{\partial s\left(\theta_{t}^{*}\right)}{\partial \theta_{t}}\right|\right)<\frac{1-\left|B_{1}\right|}{\left|A_{1}\right|} .
$$

Modelos GAS $(1,1)$ específicos para modelagem de volatilidade podem ser escritos como

$$
\begin{aligned}
& y_{t}=h\left(\theta_{t}\right) \epsilon_{t}, \\
& \theta_{t}=\omega+A_{1} s_{t-1}+B_{1} \theta_{t-1},
\end{aligned}
$$


em que $\left\{\epsilon_{t}\right\}$ é i.i.d. com $\epsilon_{t} \sim f_{\epsilon, \lambda}$ e a função $h\left(\theta_{t}\right)=h_{t}$ é suave. Como, caso geral, $\mathbb{E}\left(\epsilon_{t}\right)=0$, então

$$
s_{t}=S_{t} \nabla_{t}=-S_{t} \nabla h_{t}\left(\nabla f_{\epsilon, \lambda}\left(\epsilon_{t}\right) \epsilon_{t}+1\right),
$$

em que $\nabla h_{t}=\partial \ln h_{t} / \partial \theta_{t}$ e $\nabla f_{\epsilon, \lambda}\left(\epsilon_{t}\right)=\partial f_{\epsilon, \lambda}\left(\epsilon_{t}\right) / \partial \epsilon_{t}$. Segue que

$$
\frac{\partial s_{t}}{\partial \theta_{t}}=-\left(\frac{\partial \nabla h_{t}}{\partial \theta_{t}} S_{t}+\frac{\partial S_{t}}{\partial \theta_{t}} \nabla h_{t}\right)\left(\nabla f_{\epsilon, \lambda}\left(\epsilon_{t}\right) \epsilon_{t}+1\right) .
$$

Nesse caso, a Suposição 2.4.2 se aplica com

$$
\begin{aligned}
\eta\left(\theta_{t}\right) & =\eta_{t}=S_{t} \frac{\partial \nabla h_{t}}{\partial \theta_{t}}+\frac{\partial S_{t}}{\partial \theta_{t}} \nabla h_{t}, \\
\zeta_{1}\left(\epsilon_{t}\right) & =\zeta_{1, t}=-\nabla f_{\epsilon, \lambda}\left(\epsilon_{t}\right) \epsilon_{t}-1, \\
\zeta_{2}\left(\epsilon_{t}\right) & =\zeta_{2, t}=0 .
\end{aligned}
$$

Uma região de estacionariedade e ergodicidade pode ser obtida contanto que $\eta\left(\theta_{t}\right)$ seja uniformemente limitada e $\mathbb{E}\left(\left|\zeta_{1, t}\right|\right)<\infty$. No entanto, isso nem sempre acontece. Por exemplo, se

$$
y_{t}=h_{t} \epsilon_{t}, \quad h_{t}=\theta_{t}^{1 / 2}, \quad \epsilon_{t} \sim N(0,1), \quad S_{t}=1,
$$

tem-se que $\eta_{t}=\frac{1}{2} \theta_{t}^{-2}$, que não é limitada. Logo, não é possível garantir a existência de uma região de estacionariedade e ergodicidade não-degenerada para tal modelo GAS.

No entanto, alterando a função de ponderação para

$$
S_{t}=\mathcal{I}_{t}^{-1},
$$

o que obtemos é o clássico modelo GARCH gaussiano no caso de $\epsilon_{t} \sim N(0,1)$, conforme verificado na Seção 2.2, com

$$
\theta_{t}=\omega+A_{1} y_{t-1}^{2}+\left(B_{1}-A_{1}\right) \theta_{t-1}
$$

Obtém-se

$$
s_{t}=\theta_{t}\left(\epsilon_{t}^{2}-1\right), \quad \frac{\partial s_{t}}{\partial \theta_{t}}=\epsilon_{t}^{2}-1
$$

Nesse caso,

$$
S_{t}=2 \theta_{t}^{2}, \quad \frac{\partial S_{t}}{\partial \theta_{t}}=4 \theta_{t}, \quad \nabla h_{t}=\frac{1}{2 \theta_{t}}, \quad \frac{\partial \nabla h_{t}}{\partial \theta_{t}}=-\frac{1}{2 \theta_{t}^{2}}
$$

Logo, $\eta_{t}$ e, como $s_{t}=\zeta_{1, t} \theta_{t}+\zeta_{2, t}=\theta_{t}\left(\epsilon_{t}^{2}-1\right)$, obviamente os valores esperados de $\zeta_{1, t}$ e $\zeta_{2, t}$ são finitos.

Pela parametrização, $\theta_{t}$ é a variância de $y_{t}$. No modelo GAS referido, considerando a distribuição normal padrão para $\epsilon_{t}$, tem-se que

$$
s_{t}=\theta_{t}\left(\epsilon_{t}^{2}-1\right), \quad \frac{\partial s_{t}}{\partial \theta_{t}}=\epsilon_{t}^{2}-1
$$

Logo,

$$
\mathbb{E}\left(\sup _{\theta_{t}^{*}}\left|B_{1}+A_{1} \frac{\partial s\left(\theta_{t}^{*}\right)}{\partial \theta_{t}}\right|\right)=\mathbb{E}\left(\left|B_{1}+A_{1}\left(\epsilon_{t}^{2}-1\right)\right|\right)=B_{1}+A_{1}(1-1)=B_{1} .
$$

Para que o modelo seja estacionário, deve-se ter

$$
\mathbb{E}\left(\sup _{\theta_{t}^{*}}\left|B_{1}+A_{1} \frac{\partial s\left(\theta_{t}^{*}\right)}{\partial \theta_{t}}\right|\right)<1 \Rightarrow B_{1}<1
$$


A condição de estacionariedade conhecida para o modelo GARCH é que a soma dos coeficientes dos dois somatórios da expressão (2.13) deve ser menor que um (Bollerslev, 1986). Ou seja, em um modelo $\operatorname{GARCH}(1,1), A_{1}+\left(B_{1}-A_{1}\right)=B_{1}<1$, o que coincide com a condição obtida via (2.44) para o modelo GAS em questão.

\subsection{Estimação por máxima verossimilhança}

Uma propriedade conveniente de modelos guiados por observações é a maneira relativamente simples de estimação dos parâmetros do modelo pelo método de máxima verossimilhança. Tal propriedade se aplica aos modelos GAS. O cálculo da função de log-verossimilhança de um modelo GAS, quando possui forma fechada, é direto. Requer somente a implementação da equação de evolução do parâmetro que evolui no tempo do modelo GAS (2.3) e do cálculo da log-verossimilhança, para um valor particular $\boldsymbol{\xi}^{*}$ de $\boldsymbol{\xi}$.

É possível formular recursões para calcular o gradiente da verossimilhança com respeito ao vetor de parâmetros estáticos $\boldsymbol{\xi}$. Recursões de gradiente para o modelo GARCH foram desenvolvidas por Fiorentini et al. (1996) e podem ser adaptadas ao modelo GAS de maneira similar.

As derivadas analíticas resultantes podem ser difíceis de calcular em casos específicos, principalmente por causa da não-linearidade introduzida no uso da função score. Nesses casos, a maximização da verossimilhança deve ser baseada em derivadas numéricas.

Os cálculos podem ser custosos, especialmente se a função densidade da distribuição condicional for complicada. Como abordaremos o uso de distribuições estáveis, que não possuem formas fechadas para suas funções densidade em geral, esse tópico será discutido mais detalhadamente ao longo deste trabalho.

Os erros padrão dos estimadores podem ser calculados baseados na inversa da matriz Hessiana da log-verossimilhança avaliada no ponto ótimo. Blasques et al. (2014b) demonstraram que, sob condições de regularidade adequadas, o estimador de máxima verossimilhança $\hat{\boldsymbol{\xi}}$ de $\boldsymbol{\xi}$ é consistente e satisfaz

$$
\sqrt{n}(\hat{\boldsymbol{\xi}}-\boldsymbol{\xi}) \stackrel{D}{\Rightarrow} Z \sim N\left(\mathbf{0}, \boldsymbol{H}^{-1}\right)
$$

em que $\boldsymbol{H}=\lim _{n \rightarrow \infty} \mathbb{E}\left[(\partial l / \partial \boldsymbol{\xi})\left(\partial l / \partial \boldsymbol{\xi}^{\prime}\right)\right] / n$ e $l=\sum_{t=1}^{n} l_{t}$, em que $l_{t}=\ln f_{t}$. A notação "D $\Rightarrow$ denota convergência em distribuição. Os resultados foram estabelecidos para modelos GAS bem especificados e mal especificados. No caso de modelos mal especificados, os resultados assintóticos são válidos com respeito a um parâmetro pseudo-verdadeiro que minimiza a divergência de KullbackLeibler entre a verdadeira e desconhecida medida de probabilidade e a medida imposta pelo modelo. 


\section{Capítulo 3}

\section{Distribuições estáveis}

As distribuições estáveis são consideradas generalizações importantes da distribuição normal, sendo definidas como a classe de distribuições cujas famílias de localização-escala são fechadas sob convolução. A distribuição normal, no caso, é obtida quando o par localização-escala é a média e a variância. Do ponto de vista teórico, pode ser mostrado que as distribuições estáveis fornecem a classe de distribuições limites da soma de variáveis independentes (Gnedenko e Kolmogorov, 1968). Do ponto de vista prático, distribuições estáveis são objetos de grande interesse, pois permitem assimetria e regulagem do peso das caudas (Buckle, 1995). As distribuições normal, de caudas leves, e a Cauchy, de caudas pesadas, estão entre seus casos particulares.

O uso da distribuição normal limita o peso das caudas da distribuição. Quando o conjunto de dados possui caudas pesadas, tal fator pode ser um problema. Essa característica muitas vezes leva ao uso da distribuição $t$-Student ao invés da normal como distribuição condicional. A $t$-Student permite caudas mais pesadas que as da normal, mas, ao contrário da normal, não possui a desejável propriedade de estabilidade, presente nas distribuições estáveis. Dessa forma, tais distribuições têm domínios de atração e fornecem boas aproximações para um grande número de distribuições. A família de distribuições estáveis, que inclui a distribuição normal como caso particular, é uma candidata natural para modelagem de dados de caudas pesadas (Panorska et al., 1995).

As distribuições estáveis têm sido propostas como modelos para muitos tipos de sistemas físicos e econômicos. Nolan (2001) destaca que há três principais razões para o uso de uma distribuição estável para descrever um sistema:

- quando há razões sólidas e teóricas para que se espere um modelo não-gaussiano e estável, isto é, reflexão de um espelho rotatório levando a uma distribuição de Cauchy, tempos de chegadas de um movimento Browniano levando a uma distribuição de Lévy, o campo gravitacional de estrelas levando a uma distribuição de Holtsmark;

- o Teorema Limite Central Generalizado, que diz que a única distribuição limite não-trivial possível para somas de termos i.i.d. normalizados é a estável. Pode-se argumentar que muitas quantidades observadas são somas de pequenas outras quantidades - preço de ativos, ruído de um sistema de comunicação etc., e nesses casos um modelo estável deveria ser usado para descrever tais sistemas; e

- argumento empírico: muitos conjuntos de dados exibem caudas pesadas e assimetria.

A falta de fórmulas analíticas fechadas para densidades e funções de distribuição para as distribuições estáveis, à exceção de alguns casos notáveis (normal, Cauchy e Lévy) foi por muitos anos um empecilho para seu uso. No entanto, a evolução de métodos computacionais permitiu, principalmente nos últimos anos, o crescente uso prático de tais distribuições (Nolan, 2001).

Apesar de sua conexão com a distribuição normal, a família de distribuições estáveis não atraiu grande interesse dos matemáticos de teoria da probabilidade por muito tempo. As reservas davam-se por dois motivos: pela suposta falta de aplicação e pela dificuldade dos cálculos envolvidos. 
Em relação a aplicações, houve, principalmente a partir da década de 1960, um notável crescimento no interesse pelas distribuições estáveis, devido à sua aplicação em modelos sócio-econômicos (Zolotarev, 1986). Mandelbrot (1963) foi talvez o primeiro a avaliar a adequação de distribuições estáveis a séries de variações de preço em um trabalho notável, devido às dificuldades de se lidar com os cálculos de tais distribuições à epoca. Ele percebeu que algumas séries com as quais trabalhou mostravam evidência de ausência de segundo momento. Também argumentou que, até aquele momento, os estudos negligenciavam os aspectos leptocúrticos das distribuições de dados financeiros. A presença dessas características, segundo ele e Fama (1965), era inquestionável a tais tipos de séries, e com as quais distribuições estáveis conseguiam lidar de maneira muito mais adequada que a distribuição normal. Fama (1963) e Fama (1965) deram continuidade ao trabalho e, após estudos detalhados, concluíram que "foi demonstrado que a primeira diferença de preços de ações parece seguir distribuições estáveis com expoente característico $\alpha<2$ (ou seja, de variância infinita)". Na época, o estudo acerca de estimação de distribuições estáveis era praticamente inexistente. Fama e Roll (1968) e Fama e Roll (1971) forneceram estimativas básicas de parâmetros para casos simétricos. DuMouchel (1975) forneceu alguns resultados inferenciais relacionados às distribuições estáveis.

Em relação a dificuldade dos cálculos, a falta de uma expressão analítica para as funções densidade de probabilidade limitou por muito tempo o uso de tais distribuições, pois a verossimilhança em geral não pode ser representada em uma forma analítica tratável (Buckle, 1995). Nos últimos anos, o desenvolvimento de métodos computacionais ampliou as possibilidades de uso das distribuições estáveis. No Capítulo 4 serão detalhados aspectos do cálculo envolvendo as densidades e suas derivadas.

\subsection{História}

As leis da família estável surgiram devido ao trabalho de Paul Lévy na década de 1920. Mas tal surgimento se deve à atenção que Siméon Denis Poisson e Augustin-Louis Cauchy deram, no século anterior, à distribuição com densidade

$$
p_{\lambda}(x)=\frac{\lambda}{\pi\left(x^{2}+\lambda^{2}\right)}, \quad \lambda>0, x \in \mathbb{R},
$$

que tem a propriedade

$$
p_{\lambda_{1}} * p_{\lambda_{2}}=p_{\lambda}
$$

em que $\lambda$ é unicamente determinado por $\lambda_{1}$ e $\lambda_{2}$. Cauchy descobriu que a densidade $p_{\lambda}$ está incluída (para $\alpha=1$ ) no conjunto $\left\{f_{\lambda}^{(\alpha)}\right\}$ de funções cuja função característica tem a forma

$$
\exp \left(-\lambda|t|^{\alpha}\right), \quad \alpha>0, \lambda>0 .
$$

No início do século seguinte, o húngaro George Pólya provou que $f_{\lambda}^{(\alpha)} \geq 0$ para $0<\alpha<1$ e demonstrou que entre as funções $f_{\lambda}^{(\alpha)}$ há somente uma que é densidade de uma distribuição com variância finita. Para tanto, o valor de $\alpha$ correspondente é 2 , o que corresponde a uma distribuição normal. O fato que as funções $f_{\lambda}^{(\alpha)}$ são densidades de probabilidade apenas para $0<\alpha \leq 2$ foi estabelecido por Paul Lévy em 1925.

O conceito de distribuições estáveis tomou forma em 1937 com os trabalhos de Lévy e de Alexander Kintchine, que caracterizaram as distribuições estáveis após estudar somas normalizadas de variáveis aleatórias i.i.d. A partir de então, ficou claro que, à exceção de alguns casos, as distribuições estáveis não possuem expressões explícitas para suas funções densidade e de distribuição.

Em 1919, alguns anos antes do aparecimento do trabalho de Lévy que introduzia o conceito de lei estável, o astrônomo norueguês Johan Peter Holtsmark publicou um artigo no qual ele descobriu um princípio probabilístico que guiava as flutuações aleatórias do campo gravitacional de estrelas no espaço, sob certas suposições naturais. Mais tarde, soube-se que a distribuição descoberta por Holtsmark, que hoje leva seu nome, é uma distribuição estável. 
Nos anos 1960, principalmente devido aos trabalhos pioneiros já citados de Mandelbrot e Fama, as distribuições estáveis começaram a atrair a atenção de acadêmicos da área de economia, biologia, sociologia e linguística matemática.

Após um período inicial de interesse, os estudos que relacionavam distribuições estáveis a dados financeiros diminuíram. Tal fato ocorreu principalmente pelas dificuldades em lidar com tais distribuições, pois em geral funções densidade não estão disponíveis em forma fechada. Cálculos considerados irrisórios para os computadores de hoje em dia, como estimação por mínimos quadrados, na época eram muito custosos. Que dirá a estimação de parâmetros de uma distribuição cuja densidade não possui fórmula analítica fechada. Cootner (1964) apud Frain (2009) comentou, com ironia, as sugestões de Mandelbrot para o uso de distribuições estáveis: "Mandelbrot, como o primeiro ministro Churchill, nos promete não somente utopia, mas sangue, suor, trabalho árduo e lágrimas."

Outro fator que relegou as distribuições estáveis a um segundo plano na área financeira foi o desenvolvimento de trabalhos muito bem sucedidos que se utilizaram da distribuição normal, rendendo inclusive prêmios Nobel a Harry Markowitz, Merton Miller, William Sharpe, Roberto Merton, Myron Scholes e Robert Engle. Uma opinião equivocada circulou por muito tempo de que considerar distribuições estáveis invalidaria não apenas tais trabalhos, mas também a maioria do trabalho econométrico desenvolvido até então. Com o avanço dos métodos computacionais nos últimos anos e a crescente facilidade para a utilização das distribuições estáveis, o interesse em tais processos tem aumentado e a tendência é que continue a crescer (Frain, 2009).

Segundo Zolotarev (1986), alguns dos principais pesquisadores no desenvolvimento das distribuições estáveis são: Gnedenko e Kolmogorov, que nos anos 1950 exploraram o fato de que distribuições estáveis podem ser vistas como uma classe de distribuções limite de somas de variáveis aleatórias independentes, como demonstrado por Lévy nos anos 20 (Gnedenko e Kolmogorov, 1968); Press (1972), que introduziu a estimação dos parâmetros baseada na função característica amostral; DuMouchel (1973), que estudou possibilidades para a estimação dos parâmetros por máxima verossimilhança; Koutrouvelis (1981), que aperfeiçoou o método de Press e hoje é um dos mais utilizados; e Fama e Roll (1971) e McCulloch (1986), que exploraram a estimação dos parâmetros via quantis.

Com o avanço da informática, métodos numéricos vêm sendo cada vez mais utilizados, tanto para estimação quanto para simulação. Nos dias de hoje, John Nolan é um dos pesquisadores mais ativos na área e desenvolveu em 1997 um método e um software para estimação por máxima verossimilhança (Nolan, 2001).

Para mais detalhes acerca do desenvolvimento histórico das distribuições estáveis, ver Zolotarev (1986), Samorodnitsky e Taqqu (1994) e Kogon e Williams (1998).

\subsection{Definição}

Há diversas maneiras, que são equivalentes, de definir uma distribuição estável. A primeira que será apresentada a seguir é, por tradição, usada particularmente como definição. Tal tradição remonta dos trabalhos de Lévy e Kintchine (Zolotarev, 1986).

Definição 3.2.1 Uma variável aleatória $X$ é dita ter distribuição estável se para quaisquer números positivos a e b há um número positivo c e um real d tais que

$$
a X_{1}+b X_{2} \stackrel{D}{=} c X+d
$$

em que $X_{1}$ e $X_{2}$ têm a mesma distribuiçẫo de $X$, e "D” denota igualdade em distribuição. X é chamada estritamente estável se (3.4) é válida com $d=0$.

Definição 3.2.2 (equivalente à Definição 3.2.1) Uma variável aleatória $X$ é dita ter distribuição 
estável se para qualquer $n \geq 2$ existe um número positivo $c_{n}$ e um real $d_{n}$ tais que

$$
X_{1}+X_{2}+\cdots+X_{n} \stackrel{D}{=} c_{n} X+d_{n}
$$

em que $X_{1}, X_{2}, \ldots, X_{n}$ têm a mesma distribuição de $X$.

Em (3.5) temos, necessariamente, $c_{n}=n^{1 / \alpha}$, para algum $0<\alpha \leq 2$, ou seja, $X_{1}+X_{2}+\cdots+X_{n} \sim$ $n^{1 / \alpha} X+d_{n}$. Este é o mesmo $\alpha$ que logo será descrito.

Definição 3.2.3 (equivalente às Definições 3.2.1 e 3.2.2) Uma variável aleatória $X$ é dita ter distribuiçâa estável se tem um dominio de atração, ou seja, se existe uma sequência de variáveis aleatórias i.i.d. $Y_{1}, Y_{2}, \ldots$ e sequências de números positivos $\left\{d_{n}\right\}$ e números reais $\left\{a_{n}\right\}$ tais que

$$
\frac{Y_{1}+Y_{2}+\ldots Y_{n}}{d_{n}}+a_{n} \stackrel{D}{\Longrightarrow} X .
$$

A notação “D $\Longrightarrow$ " denota convergência em distribuiçẫo.

Para mais detalhes acercas das definições, ver Samorodnitsky e Taqqu (1994).

Pela Definição 3.2.2, fica claro que uma variável aleatória com distribuição estável pode ser escrita como uma soma ponderada de duas ou mais variáveis aleatórias com a mesma distribuição. A palavra estável é utilizada porque a forma da distribuição é estável ou constante sob somas do tipo (3.4). Alguns autores utilizam o termo soma estável para enfatizar o fato que (3.4) é sobre uma soma e para distinguir tais distribuições das distribuições max-estável, min-estável, estável multiplicativa e estável geométrica. Além disso, a literatura mais antiga usa termos ligeiramente diferentes: "estável" era originalmente utilizada para o que chamamos agora de estritamente estável e "quasi-estável" era reservada para o que chamamos hoje de estável (Nolan, 2017).

É fácil verificar que a distribuição normal é estável. Se $X$ e $Y$ são variáveis aleatórias normais independentemente distribuídas com média $\mu$ e uma variância qualquer positiva, então $\forall a, b \in \mathbb{R}$ vemos que, para $d=\mu\left(a+b-\sqrt{a^{2}+b^{2}}\right)$ e $c=\sqrt{a^{2}+b^{2}}$, a variável aleatória $Z=(a X+b Y-d) / c$ tem a mesma distribuição de $X$ e de $Y$.

A seguinte definição de distribuição estável é baseada em sua função característica, e pode ser mostrado que é equivalente às anteriores.

Definição 3.2.4 Uma variável aleatória $X$ tem distribuição estável se e somente se sua função característica $\phi(t)$ pode ser escrita como

$$
\phi(t)=\mathbb{E}\left(e^{i t X}\right)= \begin{cases}\exp \left\{-\gamma^{\alpha}|t|^{\alpha}\left(1-i \beta \operatorname{sinal}^{*}(t) \tan \frac{\pi \alpha}{2}\right)+i \delta t\right\}, & \alpha \neq 1 \\ \exp \left\{-\gamma|t|\left(1+i \beta \frac{2}{\pi} \operatorname{sinal}^{*}(t) \ln |t|\right)+i \delta t\right\}, & \alpha=1\end{cases}
$$

em que

$$
\operatorname{sinal}^{*}(t)= \begin{cases}1, & t>0 \\ 0, & t=0 \\ -1, & t<0 .\end{cases}
$$

Pela Definição 3.2.4, a distribuição estável necessita de quatro parâmetros para ser descrita: um índice de estabilidade ou expoente característico $\alpha \in(0 ; 2]$, um parâmetro de assimetria $\beta \in[-1 ; 1]$, um parâmetro de escala $\gamma \geq 0$ e um parâmetro de localização $\delta \in \mathbb{R}$ ( $\beta$ é irrelevante quando $\alpha=2)$. Como $\alpha$ e $\beta$ determinam a forma da distribuição, eles podem ser considerados parâmetros de forma. Se $X$ tem distribuição estável, adotamos a seguinte notação: $X \sim S_{\alpha}(\beta, \delta, \gamma)$.

Nos anos 1920, Pólya demonstrou que, entre as distribuições estáveis, existe apenas uma com variância finita. É justamente a distribuição normal, que é obtida quando $\alpha=2$. Lévy demonstrou em 1925 que, ao se considerar $\alpha$ fora do intervalo (0;2], o que se obtém não são densidades de probabilidade (Zolotarev, 1986).

Há três casos de distribuições estáveis nos quais podem-se obter expressões analíticas fechadas para as densidades. Tais distribuições correspondem à normal, à Cauchy e à Lévy (Nolan, 2017). 
Exemplo 3.2.1 Se $\alpha=2$, a função característica da variável aleatória com distribuição estável correspondente é dada por

$$
\phi(t)=\exp \left\{i \delta t-\gamma^{2} t^{2}\right\}
$$

Essa é a função característica de uma variável aleatória com distribuição normal de média $\delta e$ variância $2 \gamma^{2}$.

Exemplo 3.2.2 Se $\alpha=1$ e $\beta=0$, a função característica da variável aleatória com distribuição estável correspondente é dada por

$$
\phi(t)=\exp \{i \delta t-\gamma|t|\} .
$$

Essa é a função característica de uma variável aleatória com distribuição de Cauchy de parâmetro de localização $\delta$ e parâmetro de escala $\gamma$. Sua função densidade é dada por

$$
f(x)=\frac{1}{\pi} \frac{\gamma}{\gamma^{2}+(x-\delta)^{2}}, x \in \mathbb{R} .
$$

Exemplo 3.2.3 Se $\alpha=1 / 2$ e $\beta=1$, a função característica da variável aleatória com distribuição estável correspondente é dada por

$$
\phi(t)=\exp \left\{i \delta t-\sqrt{\gamma|t|}\left(1-i \frac{t}{|t|} \tan \left(\frac{\pi}{4}\right)\right)\right\} .
$$

Essa é a função característica de uma variável aleatória com distribuição de Lévy de parâmetro de localização $\delta$ e parâmetro de escala $\gamma$. Sua função densidade é dada por

$$
f(x)=\sqrt{\frac{\gamma}{2 \pi}} \frac{1}{(x-\delta)^{3 / 2}} \exp \left\{-\frac{\gamma}{2(x-\delta)}\right\}, x>\delta .
$$

A Figura 3.1 exibe um gráfico dessas três densidades com $\delta=0$ e $\gamma=1$. As distribuições normal e Cauchy são simétricas e têm forma de sino. O principal fator de diferenciação é que a Cauchy tem caudas bem mais pesadas. De fato, em distribuições estáveis, quanto menor o valor de $\alpha$, mais pesadas são as caudas. Em contraste com a normal e a Cauchy, a distriubição de Lévy é altamente assimétrica, com toda a densidade de probabilidade concentrada em $x>0$, e possui uma cauda ainda mais pesada que as da Cauchy, já que o valor de $\alpha$ é menor (Nolan, 2017).

Além das distribuições normal, Cauchy, Lévy e a reflexão da distribuição de Lévy, não se conhecem expressões analíticas fechadas para densidades de distribiuições estáveis, e é improvável que existam outras distribuições de tal classe com densidades em formas fechadas. Pode-se pensar que isso seja um empecilho fundamental para a falta de aplicação das distribuições estáveis. Mas, apenas para tomar um exemplo, também não há forma fechada para a função de distribuição da normal, e existem tabelas e algoritmos computacionais para calculá-la e ela é utilizada rotineiramente em incontáveis modelos. Hoje, temos programas de computador que calculam diversas quantidades de interesse das distribuições estáveis, e com isso é possível utilizá-las em problemas práticos (Nolan, 2017). No entanto, tais cálculos não são simples e requerem muito cuidado, principalmente no que se refere a estimação de parâmetros. Zolotarev (1986), Nolan (1997) e Matsui e Takemura (2006) desenvolveram métodos eficientes para o cálculo das densidades e das funções de distribuições estáveis, que serão discutidos no Capítulo 4.

\subsection{Propriedades}

Cada parâmetro das distribuições estáveis tem um significado prático. A Figura 3.2 justifica $\delta$, $\gamma, \beta$ e $\alpha$ como os parâmetros de localização, escala, assimetria e peso das caudas, respectivamente, através da comparação de diferentes funções densidade. 


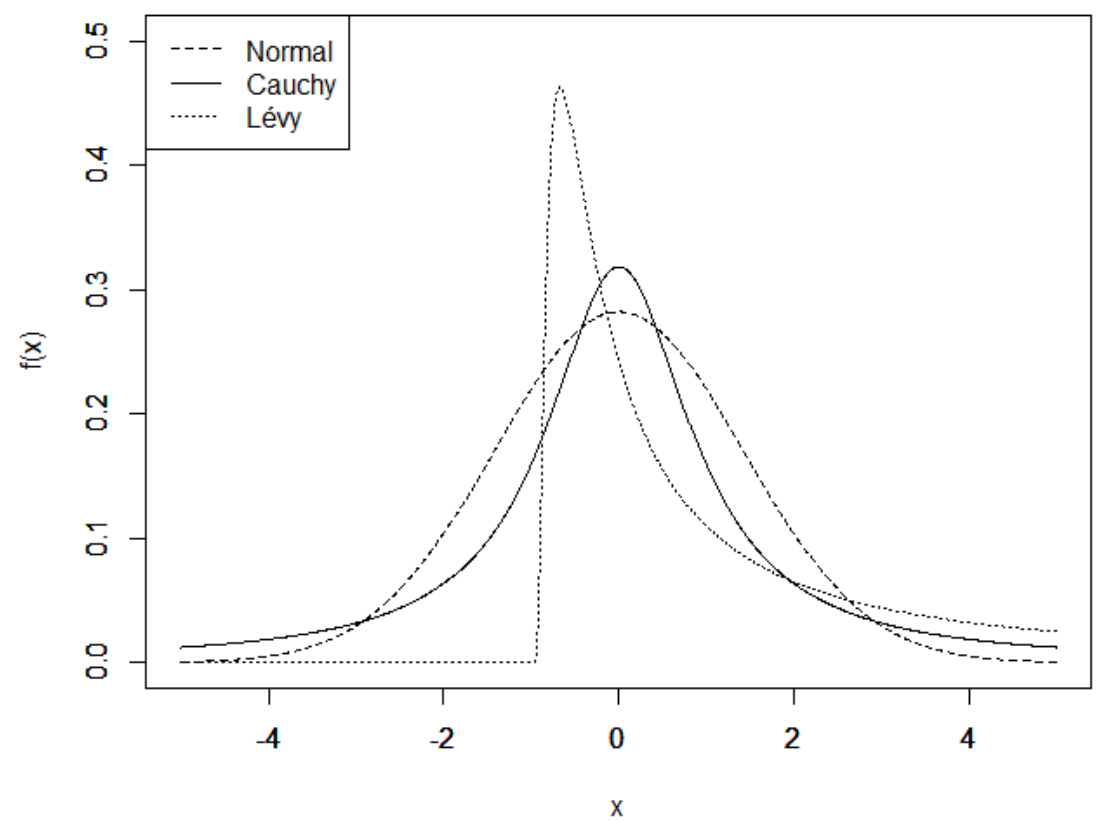

Figura 3.1: Funções densidade de probabilidade de distribuições estáveis: normal $(\alpha=2, \beta=0, \delta=0$, $\gamma=1)$, Cauchy $(\alpha=1, \beta=0, \delta=0, \gamma=1)$ e Lévy $(\alpha=1 / 2, \beta=1, \delta=0, \gamma=1)$

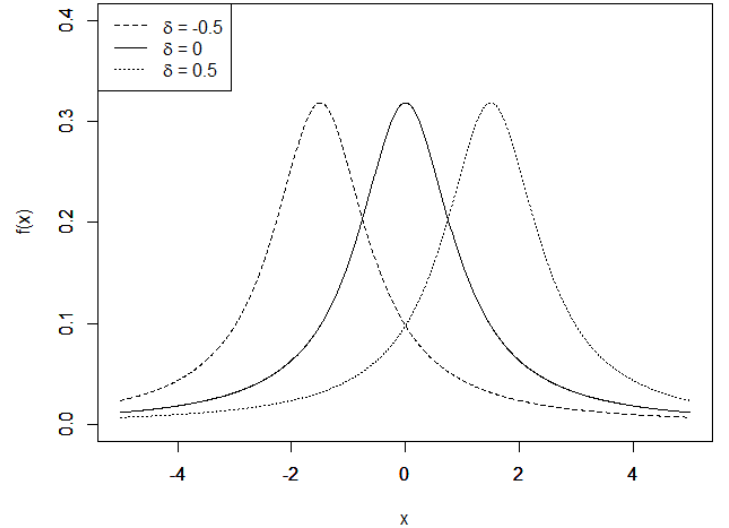

(a) Variando $\delta(\alpha=1, \beta=0, \gamma=1)$

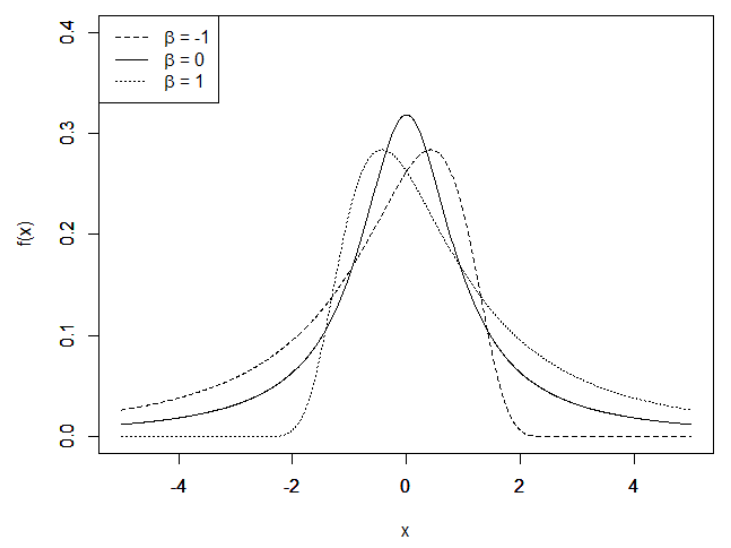

(c) Variando $\beta(\alpha=1, \delta=0, \gamma=1)$

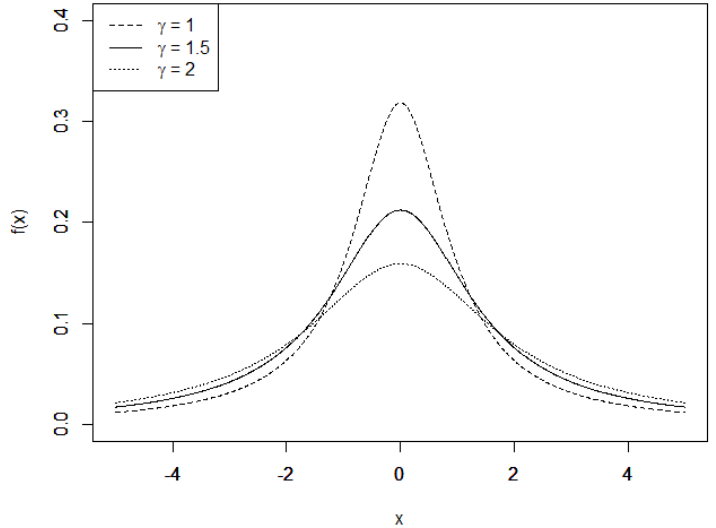

(b) Variando $\gamma(\alpha=1, \beta=0, \delta=0)$

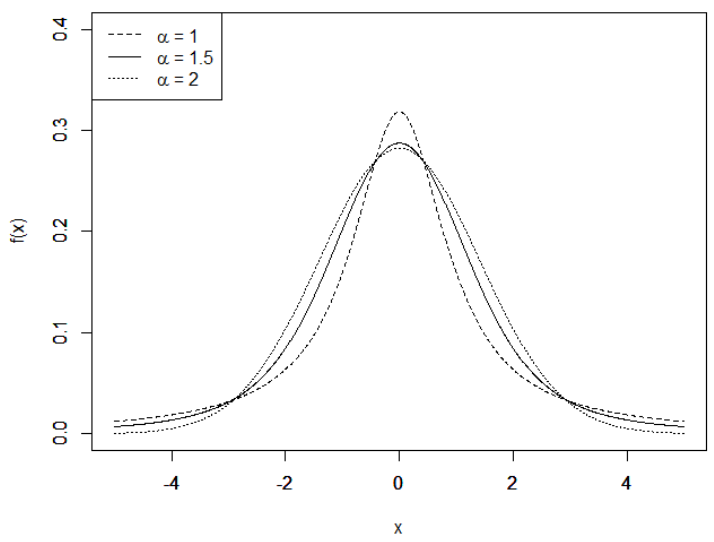

(d) Variando $\alpha(\beta=0, \delta=0, \gamma=1)$

Figura 3.2: Comparação de funções densidade de distribuições estáveis em termos de variações de seus parâmetros 
As seguintes propriedades justificam de forma teórica as características dos parâmetros. As Propriedades 3.3.1-3.3.4 podem ser facilmente verificadas com o uso da função característica. Para mais detalhes, ver Samorodnitsky e Taqqu (1994)

Propriedade 3.3.1 Seja $a \in \mathbb{R}$. Se $X \sim S_{\alpha}(\beta, \delta, \gamma)$, então $X+a \sim S_{\alpha}(\beta, \delta+a, \gamma)$.

Propriedade 3.3.2 Sejam $X \sim S_{\alpha}(\beta, \delta, \gamma)$ e b uma constante não nula. Então $b X \sim S_{\alpha}(\operatorname{sinal}(b) \beta$, $b \delta,|b| \gamma)$ se $\alpha \neq 1$ e $b X \sim S_{\alpha}(\operatorname{sinal}(b) \beta, b \delta-2 b \gamma \beta \ln |b| / \pi,|b| \gamma)$ se $\alpha=1$.

Propriedade 3.3.3 Seja $X \sim S_{\alpha}(\beta, 0, \gamma)$. Para todo $0<\alpha<2,-X \sim S_{\alpha}(-\beta, 0, \gamma)$.

Dizemos que $X$ tem distribuição simétrica se $X$ tem a mesma distribuição de $-X$. Isso ocorre se e somente se sua função característica é real, o que é o caso se $\beta=0$ e $\delta=0$.

A distribuição $S_{\alpha}(\beta, \delta, \gamma)$ é dita ser assimétrica à direita se $\beta>0$ e à esquerda se $\beta<0$. É dita ser totalmente assimétrica à direita se $\beta=1$ e totalmente assimétrica à esquerda se $\beta=-1$.

Pelas Propriedades 3.3.1 e 3.3.2, vemos que qualquer distribuição estável pode ser obtida através de mudanças de localização e escala de alguma $Z \sim S_{\alpha}(\beta, 0,1)$. Dizemos que $Z$ é uma variável aleatória com distribuição estável padrão.

De fato, as Propriedades mostram que qualquer distribuição estável pode ser obtida através de mudanças de localização e de escala de alguma $Z \sim S_{\alpha}(\beta, 0,1)$.

Propriedade 3.3.4 (consequência das Propriedades 3.3.1 e 3.3.2) Seja $Z \sim S_{\alpha}(\beta, 0,1)$. Então, para $\delta \in \mathbb{R}$ e $\gamma>0$,

$$
X= \begin{cases}\gamma Z+\delta, & \alpha \neq 1 \\ \gamma Z+\frac{2}{\pi} \gamma \beta \ln \gamma+\delta, & \alpha=1\end{cases}
$$

tem distribuição estável $S_{\alpha}(\beta, \delta, \gamma)$.

Propriedade 3.3.5 Seja $X \sim S_{\alpha}(\beta, \delta, \gamma) \operatorname{com} 0<\alpha<2$. Então

$$
\begin{aligned}
\lim _{x \rightarrow \infty} x^{\alpha} \mathbb{P}(X>x) & =C_{\alpha} \frac{1+\beta}{2} \gamma^{\alpha} \mathrm{e} \\
\lim _{x \rightarrow \infty} x^{\alpha} \mathbb{P}(X<-x) & =C_{\alpha} \frac{1-\beta}{2} \gamma^{\alpha},
\end{aligned}
$$

em que

$$
C_{\alpha}=\left(\int_{0}^{\infty} x^{-\alpha} \operatorname{sen} x d x\right)^{-1}= \begin{cases}\frac{1-\alpha}{\Gamma(2-\alpha) \cos (\pi \alpha / 2)}, & \text { se } \alpha \neq 1, \\ \frac{2}{\pi}, & \text { se } \alpha=1\end{cases}
$$

Ou seja, se $\alpha<2$, as probabilidades das caudas se comportam como $x^{-\alpha}$. Quanto menor o valor de $\alpha$, mais pesadas são as caudas. Quando comparamos as caudas da normal $(\alpha=2)$, da Cauchy $(\alpha=1)$ e da Lévy $(\alpha=1 / 2)$, percebemos que a cauda da Lévy é mais pesada, seguida pelas da Cauchy e pelas da normal.

Se $\alpha<2$, as caudas da distribuição decaem como uma função potência. Isso significa que tal variável aleatória exibe muito mais variabilidade que uma normalmente distribuída, ou seja, é muito mais provável valores distantes da mediana serem observados. A alta variabilidade das distribuições estáveis é uma das razões pelas quais elas têm um papel importante em modelagem (Samorodnitsky e Taqqu, 1994).

Uma consequência das caudas pesadas é que nem sempre todos os momentos existem. Em muitos problemas estatísticos, a esperança e a variância são utilizadas para descrever a distribuição dos dados. No entanto, esse pode não ser o caso para distribuições de caudas pesadas, pois as expressões envolvendo integrais podem divergir. Por isso, é útil em alguns casos utilizar momentos fracionários, $\mathbb{E}\left[|X|^{p}\right]$, em que $p \in \mathbb{R}$. A única distribuição estável que possui todos os momentos é aquela para a qual $\alpha=2$, que corresponde à distribuição normal. Se $0<\alpha<2, \mathbb{E}\left[|X|^{p}\right]$ é finita para $0<p<\alpha$ 
e infinita para $p \geq \alpha$. Em outras palavras, se $0<\alpha<2$, a distribuição correspondente não tem segundo momento finito (Nolan, 2017).

Se $\alpha \leq 1, \mathbb{E}[|X|]=+\infty$, e se $1<\alpha<2$ a média é dada pela seguinte propriedade (Nolan, 2017):

Propriedade 3.3.6 Se $1<\alpha<2$, a média de $X \sim S_{\alpha}(\beta, \delta, \gamma)$ é

$$
\mathbb{E}[X]=\delta-\beta \gamma \tan \frac{\pi \alpha}{2} .
$$

Propriedade 3.3.7 (Matsui e Pawlas, 2016) Se $Z \sim S_{\alpha}(\beta, 0,1)$ (distribuição estável padrão), para $0<\kappa<\alpha-1$, o momento fracionário de ordem $1+\kappa$ é dado por

$$
\begin{aligned}
\mathbb{E}\left[Z^{1+\kappa}\right] & =\frac{\kappa \Gamma\left(1-\frac{1+\kappa}{\alpha}\right)}{\operatorname{sen}\left(\frac{\kappa \pi}{2}\right) \Gamma(1-\kappa)}\left(1+\rho^{2}\right)^{\frac{1+\kappa}{2 \alpha}-\frac{1}{2}} \\
& \times\left\{\cos \left[\left(1-\frac{1+\kappa}{\alpha}\right) \arctan \rho\right]+\rho \operatorname{sen}\left[\left(1-\frac{1+\kappa}{\alpha}\right) \arctan \rho\right]\right\},
\end{aligned}
$$

em que $\rho=\beta \tan \frac{\pi \alpha}{2}$. Para se obter o momento fracionário de uma variável aleatória com distribuição estável não-padrão, basta utilizar as propriedades de localização-escala descritas anteriormente.

\subsection{Densidades e funções distribuição}

Se por um lado não existem fórmulas explícitas para as funções densidade das distribuições estáveis no caso geral, por outro lado muito se sabe sobre suas propriedades teóricas. Para mais detalhes acerca das seguintes propriedades, ver Nolan (2017).

Propriedade 3.4.1 Todas as variáveis aleatórias (não degeneradas) com distribuição estável são contínuas com função densidade infinitamente diferenciável.

Propriedade 3.4.2 O suporte de uma distribuição estável é $(-\infty, \infty)$ se $|\beta| \neq 1$ ou $\alpha \geq 1,[\delta, \infty)$ se $\beta=1$ e $\alpha<1$ e $(-\infty, \delta]$ se $\beta=-1$ e $\alpha<1$.

Propriedade 3.4.3 Todas as densidades de distribuiçôes estáveis são unimodais.

Propriedade 3.4.4 Para quaisquer $\alpha$ e $\beta, Z \sim S_{\alpha}(\beta, 0,1)$,

$$
Z(\alpha,-\beta)=-Z(\alpha, \beta)
$$

Logo, a densidade e a função de distribuição de uma variável aleatória $Z(\alpha, \beta)$ satisfazem $f(x \mid \alpha, \beta)=$ $f(-x \mid \alpha,-\beta)$ e $F(x \mid \alpha, \beta)=1-F(-x \mid \alpha,-\beta)$.

De maneira mais geral, se $X \sim S_{\alpha}(\beta, \delta, \gamma)$, então $-X \sim S_{\alpha}(-\beta,-\delta, \gamma)$, logo $f(x \mid \alpha, \beta, \delta, \gamma)=$ $f(-x \mid \alpha,-\beta,-\delta, \gamma)$ e $F(x \mid \alpha, \beta, \delta, \gamma)=1-F(-x \mid \alpha,-\beta,-\delta, \gamma)$.

Se $\beta=0$, a densidade e a função distribuição são simétricas ao redor de 0 e a densidade tem forma de sino. À medida que $\alpha$ diminui, o pico fica mais alto, as regiões dos flancos ficam mais baixas e as caudas ficam mais pesadas (ver Figura 3.2(d)).

Se $\beta>0$, a distribuição é assimétrica com a cauda direita da distribuição sendo mais pesada que a cauda esquerda: $\mathbb{P}(X>x)>\mathbb{P}(X<-x)$ para $x>0$ grande. $\mathrm{O}$ comportamento no caso $\beta<0$ é um reflexão do caso $\beta>0$, com a cauda esquerda sendo mais pesada.

Se $\alpha=2$, a distribuição é uma normal. Note que $\tan \frac{\pi \alpha}{2}=0$ na Definição 3.2 .4 , logo a função característica é real e a distribuição é sempre simétrica, não importando o valor de $\beta$. Em geral, se $\alpha \rightarrow 2$, todas as distribuições estáveis ficam mais próximas de serem simétricas e $\beta$ passa a ser insignificante nas aplicações (e mais difícil de ser estimado).

Apesar de muito se saber sobre as propriedades teóricas das densidades de distribuições estáveis, o fato de não haver fórmulas fechadas foi uma dificuldade para o avanço de estudos práticos acerca 
das distribuições por muitos anos. Com o avanço dos métodos computacionais, é possível calcular com precisão e de maneira eficiente as densidades e as funções de distribuição, algo que era de mais difícil acesso há algumas décadas. Tal fato fez com que a aplicabilidade das distribuições estáveis aumentasse.

Como todas as distribuições estáveis são mudanças de localização e de escala de alguma $Z \sim$ $S_{\alpha}(\beta, 0,1)$, de acordo com a Propriedade 3.3.4, apenas essas distribuições padrão são necessárias ao lidar com cálculos de densidades.

Seja $f_{\alpha, \beta}^{*}$ a função densidade da variável com distribuição estável padrão $Z$ e $\phi_{\alpha, \beta}^{*}$ sua função característica. Como a função característica está disponível para todas as distribuições estáveis, o teorema da inversão pode ser usado para obter a densidade

$$
f_{\alpha, \beta}^{*}(x)=\frac{1}{2 \pi} \int_{-\infty}^{\infty} e^{-i t x} \phi_{\alpha, \beta}^{*}(t) d t .
$$

No entanto, os cálculos podem ser trabalhosos. Nolan (1997) e Matsui e Takemura (2006) desenvolveram as fórmulas para as densidades baseadas no trabalho de Zolotarev (1986). O Capítulo 4 discute esses aspectos em mais detalhes, já que os cálculos serão necessários para a especificação do modelo proposto no Capítulo 5.

\subsection{O Teorema Limite Central Generalizado}

Teorema 3.5.1 Teorema Limite Central de Lindenberg-Lévy Seja $\left\{X_{i}\right\}, i=1, \ldots$ uma sequência de variáveis aleatórias i.i.d. com segundo momento finito. Existem sequências reais $c_{n}$ e $d_{n}$ com $c_{n}>0$ tais que

$$
\frac{S_{n}-d_{n}}{c_{n}} \stackrel{D}{\Rightarrow} Z \sim N(0,1)
$$

em que $S_{n}=X_{1}+\ldots X_{n}$ (Gnedenko e Kolmogorov, 1968).

O Teorema 3.5.1 é a versão clássica do Teorema Limite Central. A suposição de segundo momento finito é necessária. Mas, se a variância não é finita, pode-se pensar se ainda assim existe algum tipo de convergência.

Nesse caso, a variável aleatória limite, digamos $X$, deve ter a seguinte representação para $n \geq 2$ :

$$
X_{1}+\cdots+X_{n} \stackrel{D}{=} c_{n} X+d_{n} .
$$

De acordo com a Definição 3.2.1, para $c_{n}$ e $d_{n}$ adequados, essa é precisamente a definição de uma variável aleatória com distribuição estável. Lévy, em 1925, foi o primeiro a provar um Teorema Limite Central Generalizado envolvendo distribuições estáveis (Zolotarev, 1986). O Teorema Limite Central Generalizado mostra que, se a suposição de variância finita for retirada, a única distribuição limite possível é estável (Nolan, 2017).

Teorema 3.5.2 Teorema Limite Central Generalizado Uma variável aleatória não-degenerada $Y$ tem distribuição estável para algum $0<\alpha \leq 2$ se e somente se existe uma sequência i.i.d. de variáveis aleatórias $\left\{X_{i}\right\}, i=1, \ldots$ e constantes $c_{n}>0, d_{n} \in \mathbb{R}$ com

$$
\frac{S_{n}-d_{n}}{c_{n}} \stackrel{D}{\Rightarrow} Y \text {. }
$$

Para mais detalhes, ver Gnedenko e Kolmogorov (1968).

Dizemos que a variável aleatória $X$ está no domínio de atração de $Y$ se existem constantes $a_{n}>0, b_{n} \in \mathbb{R}$ com $a_{n}\left(X_{1}+\ldots X_{n}\right)-b_{n} \stackrel{D}{\Rightarrow} Z$, em que $X_{1}, X_{2}, \ldots$ são cópias i.i.d. de $X$. O Teorema 3.5.2 diz que a única distribuição não-degenerada possível com um domínio de atração é estável (Nolan, 2017). 
Em Nolan (2017) encontramos informações a respeito das constantes normalizadoras $a_{n}$ e $b_{n}$. Por exemplo, suponha que $X$ é uma variável aleatória com probabilidades caudais que satisfazem $x^{\alpha} \mathbb{P}(X>x) \rightarrow c^{+}$e $x^{\alpha} \mathbb{P}(X<-x) \rightarrow c^{-}$se $x \rightarrow \infty, \operatorname{com} c^{+}+c^{-}>0$ e $1<\alpha<2$. Então $\delta=\mathbb{E}[X]$ deve ser finita e

$$
a_{n}\left(X_{1}+\cdots+X_{n}\right)-b_{n} \stackrel{D}{\Rightarrow} Z \sim S_{\alpha}(\beta, 0,1),
$$

com

$$
a_{n}=\left(\frac{2 \Gamma(\alpha) \operatorname{sen}(\pi \alpha / 2)}{\pi\left(c^{+}+c^{-}\right)}\right)^{1 / \alpha} n^{-1 / \alpha}, \quad b_{n}=n a_{n} \delta, \quad \beta=\frac{c^{+}-c^{-}}{c^{+}+c^{-}} .
$$

Nesse caso, a taxa na qual as probabilidades caudais de $X$ decaem determina o índice $\alpha$ e os pesos relativos das caudas direita e esquerda determinam a assimetria $\beta$.

Em outras palavras, o Teorema 3.5.2 generaliza o Teorema Limite Central no sentido que a distribuição limite não é normal, mas estável. Obviamente o Teorema Limite Central clássico é um caso particular, já que a distribuição normal é uma distribuição estável.

Exemplo 3.5.1 Sejam $X_{1}, X_{2}, X_{3}, \ldots$ i.i.d. com distribuição de Cauchy de parâmetro de escala $\lambda$. Sua função densidade é dada por

$$
f_{\lambda}(x)=\frac{\lambda}{\pi\left(x^{2}+\lambda^{2}\right)}, \lambda>0, x \in \mathbb{R} .
$$

A função característica de $X_{i}, i=1,2, \ldots$, é dada por $\phi(t)=e^{-\lambda|t|}$. Se a variável aleatória tivesse segundo momento finito, $\bar{X}=\frac{S_{n}}{n}=\frac{\sum_{i=1}^{n} X_{i}}{n}$ teria distribuiçãa aproximadamente normal para $n$ grande. Como não é o caso, pelo Teorema 3.5.2, é esperado que $\bar{X}$ convirja em distribuiçẫo para uma variável aleatória com distribuição estável. De fato,

$$
\phi_{\bar{X}}(t)=\phi_{S_{n}}\left(\frac{t}{n}\right)=\prod_{i=1}^{n} \exp \left\{-\lambda \frac{|t|}{n}\right\}=\left(\exp \left\{-\lambda \frac{|t|}{n}\right\}\right)^{n}=\exp \{-\lambda|t|\} .
$$

Logo, $\bar{X}$ converge em distribuição para uma variável aleatória com distribuição estável, em particular com distribuição de Cauchy.

\subsection{Estrutura de dependência}

Uma consequência das caudas pesadas das distribuições estáveis é que, conforme já mencionado, o segundo momento pode não existir. Consequentemente, a covariância entre duas variáveis aleatórias também pode não estar disponível. Dessa forma, é preciso recorrer a outras quantidades para quantificar a dependência. Kokoszka e Taqqu (1994) introduziram a função de covariação, que é baseada na função característica das variáveis aleatórias envolvidas. Como tal função sempre existe, a existência da função de covariação é garantida.

Definição 3.6.1 Função de covariação A função de covariação entre duas variáveis aleatórias $X_{1}$ e $X_{2}$ é dada por

$$
I_{X_{1}, X_{2}}\left(\xi_{1}, \xi_{2}\right)=-\ln \mathbb{E}\left[e^{i\left(\xi_{1} X_{1}+\xi_{2} X_{2}\right)}\right]+\ln \mathbb{E}\left[e^{i\left(\xi_{1} X_{1}\right)}\right]+\ln \mathbb{E}\left[e^{i\left(\xi_{2} X_{2}\right)}\right] .
$$

Se $X_{1} \sim N\left(\mu_{1}, \sigma_{1}\right), X_{2} \sim N\left(\mu_{2}, \sigma_{2}\right)$ e $\operatorname{Cov}\left(X_{1}, X_{2}\right)=\sigma_{12}$, sabendo que as funções características de $\xi_{j} X_{j}$ e de $\xi_{1} X_{1}+\xi_{2} X_{2}$ são dadas respectivamente por

$$
\begin{aligned}
\mathbb{E}\left[e^{i t \xi_{j} X_{j}}\right] & =e^{i t \xi_{j} \mu_{j}-\frac{1}{2} \xi_{j}^{2} \sigma_{j} t^{2}}, j=1,2, \\
\mathbb{E}\left[e^{i t\left(\xi_{1} X_{1}+\xi_{2} X_{2}\right)}\right] & =e^{i t\left(\xi_{1} \mu_{1}+\xi_{2} \mu_{2}\right)-\frac{1}{2}\left(\xi_{1}^{2} \sigma_{1}+\xi_{2}^{2} \sigma_{2}+2 \xi_{1} \xi_{2} \sigma_{12}\right) t^{2}},
\end{aligned}
$$


a função de covariação entre $X_{1}$ e $X_{2}$ é dada por

$$
\begin{aligned}
I_{X_{1}, X_{2}}\left(\xi_{1}, \xi_{2}\right) & =-i\left(\xi_{1} \mu_{1}+\xi_{2} \mu_{2}\right)+\frac{1}{2}\left(\xi_{1}^{2} \sigma_{1}+\xi_{2}^{2} \sigma_{2}+2 \xi_{1} \xi_{2} \sigma_{12}\right) \\
& +i \xi_{1} \mu_{1}-\frac{1}{2} \xi_{1}^{2} \sigma_{1}+i \xi_{2} \mu_{2}-\frac{1}{2} \xi_{2}^{2} \sigma_{2} \\
& =\xi_{1} \xi_{2} \sigma_{12} .
\end{aligned}
$$

Ou seja, no caso normal a função de covariação é proporcional à covariância. Ainda, $-I_{X_{1}, X_{2}}(1,-1)=$ $\operatorname{Cov}\left(X_{1}, X_{2}\right)$. Tal fato motivou Kokoszka e Taqqu (1995) a definirem a função de codiferença.

Definição 3.6.2 Função de codiferença A função de codiferença entre duas variáveis aleatórias $X_{1}$ e $X_{2}$ é dada por

$$
\tau_{X_{1}, X_{2}}=-I_{X_{1}, X_{2}}(1,-1)=\ln \mathbb{E}\left[e^{i\left(X_{1}-X_{2}\right)}\right]-\ln \mathbb{E}\left[e^{i X_{1}}\right]-\ln \mathbb{E}\left[e^{-i X_{2}}\right] .
$$

Se $X_{1}$ e $X_{2}$ são variáveis aleatórias com distribuições estáveis com $0<\alpha<2, \tau_{X_{1}, X_{2}}$ pode ser considerada uma extensão da covariância como uma medida de dependência. É fácil verificar que, se $X_{1}$ e $X_{2}$ são independentes, $\tau_{X_{1}, X_{2}}=0$.

No contexto de séries temporais, pode-se utilizar a função de codiferença para generalizar a função de autocovariância, e uma versão normalizada para generalizar a função de autocorrelação. Tais ferramentas podem ser úteis para identificação de modelos quando as séries envolvem distribuições estáveis. Esse tópico será abordado com mais detalhes no Capítulo 5.

Um estimador natural para a função de codiferença é dado por

$$
\hat{\tau}_{X_{1}, X_{2}}=\ln \left(\frac{1}{n} \sum_{j=1}^{n} e^{i\left(x_{1, j}-x_{2, j}\right)}\right)-\ln \left(\frac{1}{n} \sum_{j=1}^{n} e^{i x_{1, j}}\right)-\ln \left(\frac{1}{n} \sum_{j=1}^{n} e^{-i x_{2, j}}\right),
$$

em que $\left\{x_{1, j}, j=1, \ldots, n\right\}$ e $\left\{x_{2, j}, j=1, \ldots, n\right\}$ são amostras de tamanho $n$ observadas de $X_{1}$ e $X_{2}$, respectivamente. Stein et al. (2016) demonstraram que o estimador é consistente no contexto de processos estacionários estáveis simétricos.

\subsection{Simulação}

Nolan (2017) destaca um teorema para simulação de variáveis aleatórias com distribuição estável.

Teorema 3.7.1 Sejam $V_{1}$ e $V_{2}$ variáveis aleatórias com distribuições uniforme no intervalo $(-\pi / 2$, $\pi / 2)$ e exponencial com média 1 , respectivamente. Se $0<\alpha \leq 2$,

- a variável aleatória

$$
W= \begin{cases}\frac{\operatorname{sen}\left(\alpha V_{1}\right)}{\left(\cos V_{1}\right)^{1 / \alpha}}\left[\frac{\cos \left(V_{1}(\alpha-1)\right)}{V_{2}}\right]^{(1-\alpha) / \alpha}, & \alpha \neq 1 \\ \tan V_{1}, & \alpha=1\end{cases}
$$

tem distribuição estável padrão simétrica de expoente característico $\alpha\left(W \sim S_{\alpha}(0,0,1)\right)$; e

- para qualquer $-1 \leq \beta \leq 1$, seja $\vartheta=\arctan (\beta \tan \pi \alpha / 2) / \alpha$ para $\alpha \neq 1$. Então

$$
W= \begin{cases}\frac{\operatorname{sen} \alpha\left(\vartheta+V_{1}\right)}{\left(\cos (\alpha \vartheta) \cos V_{1}\right)^{1 / \alpha}}\left[\frac{\cos \left(\alpha \vartheta+(\alpha-1) V_{1}\right)}{V_{2}}\right]^{(1-\alpha) / \alpha}, & \alpha \neq 1 \\ \frac{2}{\pi}\left[\left(\frac{\pi}{2}+\beta V_{1}\right) \tan V_{1}-\beta \ln \left(\frac{\pi / 2-V_{2} \cos V_{1}}{\pi / 2+\beta V_{1}}\right)\right], & \alpha=1\end{cases}
$$

tem distribuição estável padrão de expoente característico $\alpha$ e parâmetro de assimetria $\beta$ ( $W \sim$ $\left.S_{\alpha}(\beta, 0,1)\right)$. 
A demonstração do teorema foi obtida por Chambers et al. (1976).

Logo, para simular de variáveis aleatórias com distribuição estável de parâmetros de localização $\delta$ e escala $\gamma$ genéricos, basta simular de variáveis com distribuição estável padrão e aplicar a Propriedade 3.3.4.

\subsection{Estimação}

Diversos métodos estão disponíveis para a estimação dos parâmetros de distribuições estáveis. Para o índice de estabilidade $\alpha$, uma das primeiras abordagens é simplesmente observar o gráfico da função de distribuição empírica dos dados observados em uma escala log-log. De acordo com Samorodnitsky e Taqqu (1994), a cauda deve se aproximar de uma linha reta com inclinação $-\alpha$ se os dados seguem uma distribuição estável.

Outra abordagem é baseada nos quantis das distribuições estáveis. Fama e Roll (1968) notaram certos padrões nos quantis tabulados de distribuições estáveis e propuseram estimativas para $\alpha, \gamma$ e $\delta$ para distribuições estáveis simétricas $(\beta=0)$. McCulloch (1986) estendeu essas ideias para o caso geral (não simétrico).

Como as expressões analíticas das funções características de distribuições estáveis são conhecidas, diversos estudos se basearam nas funções características empíricas. Press (1972) aparentemente foi o primeiro a fazê-lo. Em seguida, diversas modificações foram feitas para essa abordagens, por exemplo Koutrouvelis (1981) e Kogon e Williams (1998).

O procedimento de máxima verossimilhança também já foi muito estudado na literatura. A dificuldade para o cálculo da função de verossimilhança reside no óbvio fato de que as funções densidade não são conhecidas no caso geral para as distribuições estáveis. DuMouchel (1973) utilizou a transformada rápida de Fourier para calcular a densidade de valores centrais e expansões em séries para as caudas. Brant (1984) propôs um método para aproximar a verossimilhança utilizando a função característica.

Zolotarev (1986) detalha o quando se sabe acerca das densidades de distribuições estáveis. Baseado nesse trabalho e no de Nolan (1997), Matsui e Takemura (2006) detalham como calcular as densidades e suas derivadas, assim como a informação de Fisher, de distribuições estáveis simétricas. Para o modelo proposto nesta tese, a ser apresentado no Capítulo 5, o procedimento de estimação é desenvolvido baseado nesses cálculos e no método de Newton-Raphson relaxado. Os cálculos das densidades e de suas derivadas e da informação de Fisher são detalhados no Capítulo 4. 


\section{Capítulo 4}

\section{Cálculos referentes a distribuições estáveis simétricas}

Neste Capítulo alguns aspectos a respeito do cálculo de densidades de distribuições estáveis simétricas e suas derivadas serão explorados. Tais quantidades serão fundamentais para os modelos que serão analisados nos Capítulos seguintes.

Como não existem formas analíticas fechadas para as densidades, seus cálculos podem ser trabalhosos. A função densidade de uma variável aleatória com distribuição estável pode ser escrita em termos de uma função especial chamada de função $H$, também conhecida como função $H$ de Fox. Mathai et al. (2009) mostra como obter as densidades em alguns casos em termos da função H. No entanto, os cálculos não são eficientes ao lidar com métodos computacionais intensivos, tais como máxima verossimilhança, e o tempo de processamento é muito alto, mesmo em processadores de computadores poderosos (Sousa, 2013).

Nos dias de hoje, a melhor forma de calcular as densidades de distribuições estáveis no contexto de otimização é se basear no trabalho de Nolan (1997), que investigou a estabilidade numérica de diversas representações utilizando procedimentos de integração numérica a partir do trabalho de Zolotarev (1986). O próprio Nolan desenvolveu o programa STABLE para o cálculo de tais quantidades. No entanto, conforme Matsui e Takemura (2006), o programa apresenta em alguns casos resultados não confiáveis em casos extremos (por exemplo $\alpha$ muito próximo de 1 ou de 2). Além disso, se o objetivo é estimação por máxima verossimilhança, como é o caso deste trabalho, é desejável que as derivadas da função de log-verossimilhança sejam calculadas diretamente, e não por aproximações, como foi feito em alguns trabalhos.

Matsui e Takemura (2006), baseados nos trabalhos de Zolotarev (1986) e Nolan (1997), refinaram os cálculos e também exploraram as derivadas das densidades de forma direta, assim como a informação de Fisher, apresentando cálculos confiáveis para distribuições estáveis simétricas. Esse parece ser o último desenvolvimento significativo relacionado ao cálculo de densidades de distribuições estáveis, e são a base para o que será desenvolvido nas próximas Sessões.

Os resultados apresentados neste Capítulo em geral referem-se a distribuições estáveis com $1<\alpha \leq 2$, pois somente tal intervalo para o expoente característico será considerado no modelo proposto no Capítulo 5. Alguns resultados apresentam refinamentos em comparação aos obtidos por Matsui e Takemura (2006). Outros representam uma contribuição deste trabalho, por não estarem disponíveis na literatura. São resultados referentes a quantidades oriundas da estrutura da modelagem GAS.

Por exemplo, para o procedimento de estimação, que será detalhado no Capítulo 5, será necessário o uso da terceira derivada da função densidade de uma distribuição estável cujo cálculo é detalhado neste Capítulo e não está disponível na literatura. Outras quantidades, como as derivadas da informação de Fisher e a segunda derivada da densidade em relação ao expoente característico e ao argumento da função, também são apresentadas de forma inédita.

Os programas escritos em $\mathrm{R}$ para o cálculo das quantidades mencionadas são detalhados nas Seções A.1 e A.2 do Apêndice A. 


\subsection{Notação e resultados preliminares}

Nesta Seção são apresentadas as notações e os resultados preliminares. Como há diversas parametrizações para distribuições estáveis, é adequado definir as que serão consideradas. As parametrizações consideradas aqui seguem as de Nolan (1997) e Matsui e Takemura (2006), de acordo com as apresentadas no Capítulo 3.

Seja $f(x ; \gamma(\sigma), \alpha)$ a densidade de uma distribuição estável com parâmetro de localização $\delta=$ 0 , parâmetro de assimetria $\beta=0$ (distribuição simétrica), parâmetro de escala $\gamma(\sigma)$ e expoente característico $\alpha$ (que determina o peso das caudas). Aqui, $\gamma(\sigma)$ é uma função arbitrária, contínua e diferenciável. Se $\gamma(\sigma)=\sqrt{\sigma / 2}$, como será considerado no modelo do Capítulo 5 , a distribuição normal de média 0 e variância $\sigma$ é obtida quando $\alpha=2$. Também seja $f^{*}(x ; \alpha)$ a densidade de uma distribuição estável no caso padrão e simétrico, com parâmetro de localização $\delta=0$, parâmetro de assimetria $\beta=0$, parâmetro de escala $\gamma=1$ e expoente característico $\alpha$. Todas as funções e suas derivadas que estiverem referidas por * são relacionadas a essa específica distribuição estável padrão. Como a densidade de uma distribuição estável constitui em uma família localização-escala, tem-se

$$
f(x ; \gamma(\sigma), \alpha)=\frac{1}{\gamma(\sigma)} f^{*}\left(\frac{x}{\gamma(\sigma)} ; \alpha\right) .
$$

A seguir, a primeira derivada de $f^{*}(x ; \alpha)$ em relação a $x$ é denotada por $f^{* \prime}(x ; \alpha)$, a segunda derivada por $f^{* \prime \prime}(x ; \alpha)$ e a terceira derivada por $f^{* \prime \prime \prime}(x ; \alpha)$. As derivadas parciais com relação a $\sigma$ e $\alpha$ são indicadas por subescritos, ou seja,

$$
f_{\sigma}(x ; \gamma(\sigma), \alpha)=\frac{\partial}{\partial \sigma} f(x ; \gamma(\sigma), \alpha), \quad f_{\alpha x}(x ; \gamma(\sigma), \alpha)=\frac{\partial^{2}}{\partial \alpha \partial x} f(x ; \gamma(\sigma), \alpha)
$$

Note que, derivando (4.1) com relação a $\sigma$, tem-se

$$
\begin{aligned}
f_{\sigma}(x ; \gamma(\sigma), \alpha)= & -\frac{\gamma^{\prime}(\sigma)}{\gamma(\sigma)^{2}} f^{*}\left(\frac{x}{\gamma(\sigma)} ; \alpha\right)-x \frac{\gamma^{\prime}(\sigma)}{\gamma(\sigma)^{3}} f^{* \prime}\left(\frac{x}{\gamma(\sigma)} ; \alpha\right), \\
f_{\sigma \sigma}(x ; \gamma(\sigma), \alpha)= & \frac{2 \gamma^{\prime}(\sigma)^{2}-\gamma^{\prime \prime}(\sigma) \gamma(\sigma)}{\gamma(\sigma)^{3}} f^{*}\left(\frac{x}{\gamma(\sigma)} ; \alpha\right) \\
& -x \frac{4 \gamma^{\prime}(\sigma)^{2}-\gamma^{\prime \prime}(\sigma) \gamma(\sigma)}{\gamma(\sigma)^{4}} f^{* \prime}\left(\frac{x}{\gamma(\sigma)} ; \alpha\right)+x^{2} \frac{\gamma^{\prime}(\sigma)^{2}}{\gamma(\sigma)^{5}} f^{* \prime \prime}\left(\frac{x}{\gamma(\sigma)} ; \alpha\right), \\
f_{\sigma \sigma \sigma}(x ; \gamma(\sigma), \alpha)= & \frac{6 \gamma(\sigma) \gamma^{\prime}(\sigma) \gamma^{\prime \prime}(\sigma)-\gamma(\sigma) \gamma^{\prime \prime \prime}(\sigma)-6 \gamma^{\prime}(\sigma)^{3}}{\gamma(\sigma)^{4}} f^{*}\left(\frac{x}{\gamma(\sigma)} ; \alpha\right) \\
& +x \frac{12 \gamma(\sigma) \gamma^{\prime}(\sigma) \gamma^{\prime \prime}(\sigma)-\gamma(\sigma)^{2} \gamma^{\prime \prime \prime}(\sigma)-18 \gamma^{\prime}(\sigma)^{3}}{\gamma(\sigma)^{5}} f^{* \prime}\left(\frac{x}{\gamma(\sigma)} ; \alpha\right) \\
& +x^{2} \frac{3 \gamma(\sigma) \gamma^{\prime}(\sigma) \gamma^{\prime \prime}(\sigma)-9 \gamma^{\prime}(\sigma)^{3}}{\gamma(\sigma)^{6}} f^{* \prime \prime}\left(\frac{x}{\gamma(\sigma)} ; \alpha\right) \\
& -x^{3} \frac{\gamma^{\prime}(\sigma)^{3}}{\gamma(\sigma)^{7}} f^{* \prime \prime \prime}\left(\frac{x}{\gamma(\sigma)} ; \alpha\right) .
\end{aligned}
$$

Seja $l(x ; \gamma(\sigma), \alpha)=\ln f(x ; \gamma(\sigma), \alpha)$, e suas derivadas parciais também são indicadas por subescritos. Então, a função score é dada por $\nabla(x ; \gamma(\sigma), \alpha)=\partial \ln f(x ; \gamma(\sigma), \alpha) / \partial \sigma=l_{\sigma}(x ; \gamma(\sigma), \alpha)$, e, 
utilizando regras da cadeia e de logaritmo, tem-se

$$
\begin{aligned}
l_{\sigma}(x ; \gamma(\sigma), \alpha) & =\frac{f_{\sigma}(x ; \gamma(\sigma), \alpha)}{f(x ; \gamma(\sigma), \alpha)}, \\
l_{\sigma \sigma}(x ; \gamma(\sigma), \alpha) & =\frac{f_{\sigma \sigma}(x ; \gamma(\sigma), \alpha)}{f(x ; \gamma(\sigma), \alpha)^{2}}-\frac{f_{\sigma}(x ; \gamma(\sigma), \alpha)}{f(x ; \gamma(\sigma), \alpha)}, \\
l_{\sigma \sigma \sigma}(x ; \gamma(\sigma), \alpha) & =\frac{f_{\sigma \sigma \sigma}(x ; \gamma(\sigma), \alpha) f_{\sigma}(x ; \gamma(\sigma), \alpha)^{2}+2 f_{\sigma}(x ; \gamma(\sigma), \alpha)^{3}}{f(x ; \gamma(\sigma), \alpha)^{3}} \\
& -3 \frac{f_{\sigma}(x ; \gamma(\sigma), \alpha) f_{\sigma \sigma}(x ; \gamma(\sigma), \alpha)}{f(x ; \gamma(\sigma), \alpha)^{2}} .
\end{aligned}
$$

Para $1<\alpha<2, f^{*}(x ; \alpha)$ não possui forma analítica fechada. Como a função característica sempre existe (ver Capítulo 3), o teorema da inversão para se obter a densidade pode ser utilizado.

Teorema 4.1.1 Para $\alpha \neq 1$ e $x>0, f^{*}(x ; \alpha)$ pode ser escrita como

$$
f^{*}(x ; \alpha)=\frac{\alpha}{\pi|\alpha-1| x} \int_{0}^{\frac{\pi}{2}} W(\varphi, x) \exp (-W(\varphi, x)) d \varphi,
$$

em que

$$
W(\varphi, x)=\left(\frac{x \cos \varphi}{\operatorname{sen}(\alpha \varphi)}\right)^{\frac{\alpha}{\alpha-1}} \frac{\cos ((\alpha-1) \varphi)}{\cos \varphi} .
$$

Prova: Pelo teorema da inversão,

$$
f^{*}(x ; \alpha)=\frac{1}{2 \pi} \int_{-\infty}^{\infty} e^{-i t x} \phi_{\alpha}^{*}(t) d t
$$

em que $\phi_{\alpha}^{*}(t)$ é a função característica de uma variável aleatória com distribuição estável padrão simétrica. Conforme apresentado no Capítulo $3, \phi_{\alpha}^{*}(t)=e^{-|t|^{\alpha}}$ para $\alpha \neq 1$. Logo,

$$
f^{*}(x ; \alpha)=\frac{1}{2 \pi} \int_{-\infty}^{\infty} e^{-i t x-|t|^{\alpha}} d t=\frac{1}{\pi} \operatorname{Re} \int_{0}^{\infty} e^{i t x-t^{\alpha}} d t .
$$

Sem perda de generalidade, pois a distribuição é simétrica em torno de zero, consideramos $x>0$. No plano complexo, considere o contorno $\Gamma=\left\{t: \operatorname{Im}\left(i t x-t^{\alpha}\right)=0,0 \leq \arg t \leq \pi / 2\right\}$. $\Gamma$ é uma curva cuja equação em coordenadas polares $\left(t=r e^{i \varphi}, 0 \leq \varphi \leq \pi / 2\right)$ é obtida com

$$
\operatorname{Im}\left(i t x-t^{\alpha}\right)=0 \Rightarrow x r \cos \varphi-r^{\alpha} \operatorname{sen}(\alpha \varphi)=0 \Rightarrow r(\varphi)=\left(\frac{x \cos \varphi}{\operatorname{sen}(\alpha \varphi)}\right)^{\frac{1}{\alpha-1}}
$$

Pelo Lema 2.2.3 de Zolotarev (1986), pode-se substituir os limites de integração em (4.12) pela região determinada por $\Gamma$. Com isso,

$$
f^{*}(x ; \alpha)=\frac{1}{\pi} \operatorname{Re} \int_{\Gamma} e^{i t x-t^{\alpha}} d t=\frac{1}{\pi} \int_{\Gamma} e^{\operatorname{Re}\left(i t x-t^{\alpha}\right)} d(\operatorname{Re} t)=\frac{1}{\pi} \int_{\Gamma} e^{-W(\varphi, x)} d(r \cos \varphi),
$$

em que

$$
W(\varphi, x)=x r \operatorname{sen} \varphi+r^{\alpha} \cos (\alpha \varphi)
$$

Por (4.13),

$$
x r=r^{\alpha} \frac{\operatorname{sen}(\alpha \varphi)}{\cos \varphi} .
$$

Logo,

$$
W(\varphi, x)=r^{\alpha} \frac{\cos ((\alpha-1) \varphi)}{\cos \varphi} .
$$


Para o cálculo de $d(r \cos \varphi)$, como $x r \cos \varphi=r^{\alpha} \operatorname{sen}(\alpha \varphi)$, então, utilizando diferenciação implícita, tem-se

$$
\begin{aligned}
x \frac{d(r \cos \varphi)}{d r} & =r^{\alpha} \frac{d \operatorname{sen}(\alpha \varphi)}{d r}+\frac{d r^{\alpha}}{d r} \operatorname{sen}(\alpha \varphi)=\alpha r^{\alpha} \cos (\alpha \varphi) \frac{d \varphi}{d r}+\alpha r^{\alpha-1} \operatorname{sen}(\alpha \varphi) \\
\Rightarrow & x d(r \cos \varphi)=\alpha r^{\alpha} \cos (\alpha \varphi) d \varphi+\alpha x \cos \varphi d r .
\end{aligned}
$$

Como

$$
\alpha x \frac{d(r \cos \varphi)}{d \varphi}=\alpha x \cos \varphi \frac{d r}{d \varphi}-\alpha x r \operatorname{sen} \varphi
$$

então, substituindo em (4.18), tem-se

$$
\begin{aligned}
x d(r \cos \varphi) & =\alpha x d(r \cos \varphi)+\alpha\left(x r \operatorname{sen} \varphi+r^{\alpha} \cos (\alpha \varphi)\right) d \varphi \\
& =\alpha x d(r \cos \varphi)+\alpha W(\varphi, x) d \varphi \\
\Rightarrow d(r \cos \varphi) & =\frac{\alpha}{x(1-\alpha)} W(\varphi, x) d \varphi .
\end{aligned}
$$

Se o contorno de $\Gamma$ for seguido na direção crescente de $r$, o ângulo $\varphi$ vai de 0 a $\pi / 2$ se $\alpha<1$, e de $\pi / 2$ a 0 se $\alpha>1$. Logo, substituindo em (4.14),

$$
f^{*}(x ; \alpha)=\frac{\alpha}{\pi|\alpha-1| x} \int_{0}^{\frac{\pi}{2}} W(\varphi, x) \exp (-W(\varphi, x)) d \varphi .
$$

Para detalhes dos casos $\alpha=1$ e $\beta \neq 0$, ver Zolotarev (1986).

Para se obter o valor de $f^{*}(x ; \alpha)$, então, é preciso que se calcule a integral envolvida em (4.9). Neste trabalho, para tal cálculo, foi utilizado o comando integrate do software R, que faz uso de quadratura adaptativa baseada na quadratura de Gauss-Kronrod dentro de cada subintervalo considerado (Piessens et al., 1983). Vários testes foram feitos e os resultados são confiáveis. Há alguns poucos casos nos quais o algoritmo apresenta problemas numéricos. Para esses casos, o cálculo da densidade foi feito através da simples quadratura não-adaptativa pelo método do trapézio (Ruggiero e Lopes, 1997). Tal procedimento será utilizado para as outras integrais envolvidas nos cálculos das quantidades das Sessões seguintes.

$\operatorname{Em} x=0$,

$$
f^{*}(0 ; \alpha)=\frac{1}{\pi} \Gamma\left(1+\frac{1}{\alpha}\right),
$$

para todo $0<\alpha \leq 2$, em que $\Gamma$ é a função gama.

Obviamente a expressão (4.9) pode apresentar problemas para valores de $x$ próximos de zero ou muito elevados.

Teorema 4.1.2 Se $\alpha \neq 1, x>0$ e $x \rightarrow 0$, ent $\tilde{a} o$

$$
f^{*}(x ; \alpha)=\frac{1}{\pi \alpha} \sum_{k=0}^{\infty} \frac{\Gamma((2 k+1) / \alpha)}{(2 k) !}(-1)^{k} x^{2 k} .
$$

Se $\alpha \neq 1, x>0$ e $x \rightarrow \infty$, então

$$
f^{*}(x ; \alpha)=\frac{1}{\pi} \sum_{k=1}^{\infty} \frac{\Gamma(k \alpha+1)}{k !}(-1)^{k-1} \operatorname{sen}\left(\frac{\pi \alpha k}{2}\right) x^{-k \alpha-1} .
$$

Prova: Aqui será exibido um esboço da demonstração. Para mais detalhes, ver Pollard (1946). 
Utilizando a expressão (4.12), para $\alpha<1$,

$$
f^{*}(x ; \alpha)=\frac{1}{\pi} \operatorname{Re} \int_{0}^{\infty} e^{i t x-t^{\alpha}} d t=\frac{1}{\pi} \sum_{k=0}^{\infty} \frac{x^{k}}{k !} \operatorname{Re} \int_{0}^{\infty}(i t)^{k} e^{-t^{\alpha}} d t .
$$

Uma mudança de variável $w=t^{\alpha}$ na integral da última parcela faz com que a função Gamma apareça, e o fato da expressão considerar somente a parte real da integral faz com que as parcelas do somatório sejam nulas quando $k$ é ímpar (pois $i^{k}$ é real somente se $k$ é par). Logo, obtém-se a expressão (4.23) para $x \rightarrow 0$.

Sabendo-se que, para $\alpha>1$,

$$
f^{*}(x ; \alpha)=x^{-1-\alpha} f^{*}\left(x^{-\alpha} ; 1 / \alpha\right),
$$

utiliza-se o mesmo procedimento para a parte direita da equação (4.26) para que se obtenha a expressão (4.24) para $x \rightarrow \infty$.

A série de (4.23) não é convergente para $\alpha<1$, mas é justificada como uma expansão assintótica quando $x \rightarrow 0$. Para $1<\alpha \leq 2$, é convergente para todo $x$. De maneira similar, a série de (4.24) não é convergente para $\alpha>1$ e é justificada como uma expansão assintótica quando $x \rightarrow \infty$. Para $1<\alpha \leq 2$, é convergente para todo $x \neq 0$ (Matsui e Takemura, 2006).

Matsui e Takemura (2006) exploraram tais expansões em termos práticos, avaliando para quais valores de $x$, de $\alpha$ e de $k$ as expressões são mais adequadas.

Conforme já mencionado, o interesse são os casos nos quais $\alpha>1$. Matsui e Takemura (2006) listam suas análises para casos extremos de $\alpha$, por exemplo quando está muito próximo de 1 ou de 2. Neste trabalho, é considerado o caso $1,01<\alpha \leq 2$, e as fórmulas a serem usadas nos casos extremos são exibidas na Tabela 4.1. Uma diferença em relação ao trabalho de Matsui e Takemura é que os autores destacam que a fórmula geral (4.9) deve ser utilizada quando 1, $01<\alpha \leq 1,99999 \mathrm{e}$ $10^{-5} \leq x \leq 10^{\frac{3}{1+\alpha}}$. Após diversas checagens numéricas, verificamos que não se deve usar tal fórmula quando $x<0,6$, já que, aparentemente, neste caso, $x$ é muito próximo de 0 e a expressão não traz resultados precisos. Logo, se $1,01<\alpha \leq 1,99999$, deve-se usar a fórmula (4.23) com $k=10$ para todo $x$ em $[0 ; 0,6)$.

\begin{tabular}{|c|c|c|c|c|}
\hline$\alpha \backslash x$ & \multicolumn{2}{|c|}{$x \rightarrow 0$, fórmula (4.23) } & \multicolumn{2}{c|}{$x \rightarrow \infty$, fórmula (4.24) } \\
\hline \hline$(1,01 ; 1,99999]$ & $k=10$ & $x<0,6$ & $k=10$ & $x>10^{\frac{3}{1+\alpha}}$ \\
\hline$(1,99999 ; 2]$ & $k=85$ & $x \leq 7$ & \multicolumn{2}{|c|}{ formula (4.9) } \\
\hline
\end{tabular}

Tabela 4.1: Aproximações para a função densidade em casos extremos, $1,01<\alpha \leq 2$

\subsection{Derivadas parciais da função densidade}

\subsubsection{Primeira derivada em relação ao parâmetro de escala}

Tendo (4.3) como objetivo, é preciso calcular $f_{\sigma}(x ; \gamma(\sigma), \alpha)$. Para isso, é necessário apenas ter $f^{*}(x ; \alpha)$, que já foi calculado, e $f^{* \prime}(x ; \alpha)$. Diferenciando-se $(4.9)$ tem-se

$$
f^{* \prime}(x ; \alpha)=-\frac{1}{x} f^{*}(x ; \alpha)+\frac{\alpha^{2} \operatorname{sinal}(\alpha-1)}{\pi x^{2}(\alpha-1)^{2}} \int_{0}^{\frac{\pi}{2}} W(\varphi, x)(1-W(\varphi, x)) \exp (-W(\varphi, x)) d \varphi
$$

para $\alpha \neq 1$ e $x>0$.

Como no caso da densidade, representações alternativas da primeira derivada da densidade de uma distribuição estável padrão no caso simétrico nos casos extremos devem ser utilizadas. 
Para $x \rightarrow 0$ e $0<\alpha \leq 2, \alpha \neq 1$, diferenciando-se (4.23), tem-se:

$$
f^{* \prime}(x ; \alpha)=\frac{1}{\pi \alpha} \sum_{k=1}^{\infty} \frac{\Gamma((2 k+1) / \alpha)}{(2 k-1) !}(-1)^{k} x^{2 k-1} .
$$

De maneira similar, para $x \rightarrow \infty$ e $0<\alpha \leq 2, \alpha \neq 1$, tem-se:

$$
f^{* \prime}(x ; \alpha)=\frac{1}{\pi} \sum_{k=1}^{\infty} \frac{\Gamma(k \alpha+2)}{k !}(-1)^{k} \operatorname{sen}\left(\frac{\pi \alpha k}{2}\right) x^{-k \alpha-2} .
$$

A Tabela 4.2 traz um resumo das aproximações da primeira derivada $f^{* \prime}(x ; \alpha)$. A interpretação da Tabela 4.2 é semelhante à da Tabela 4.1. Novamente, aqui considera-se $x<0,6$ para se utilizar

\begin{tabular}{|c|c|c|c|c|}
\hline$\alpha \backslash x$ & \multicolumn{2}{|c|}{$x \rightarrow 0$, fórmula $(4.28)$} & \multicolumn{2}{|c|}{$x \rightarrow \infty$, fórmula $(4.29)$} \\
\hline$(1,01 ; 1,99999]$ & \multirow{2}{*}{$k=10$} & \multirow{2}{*}{$x<0,6$} & $k=10$ & $x>10^{\frac{3}{1+\alpha}}$ \\
\hline$(1,99999 ; 2]$ & & & \multicolumn{2}{|c|}{ fórmula (4.27) } \\
\hline
\end{tabular}
a expansão no caso de $x \rightarrow 0$, diferente de Matsui e Takemura (2006), que consideram $x<10^{-3}$.

Tabela 4.2: Aproximações para $f^{* \prime}(x ; \alpha)$ em casos extremos, $1,01<\alpha \leq 2$

\subsubsection{Segunda derivada em relação ao parâmetro de escala}

Diferenciando-se (4.27) tem-se

$$
\begin{aligned}
f^{* \prime \prime}(x ; \alpha)= & \frac{1}{\alpha-1} \frac{1}{x^{2}} f^{*}(x ; \alpha)+\frac{3-2 \alpha}{\alpha-1} \frac{1}{x} f^{* \prime}(x ; \alpha) \\
& -\frac{\alpha^{3} \operatorname{sinal}(\alpha-1)}{\pi(\alpha-1)^{3} x^{3}} \int_{0}^{\frac{\pi}{2}} W^{2}(\varphi, x)(2-W(\varphi, x)) \exp (-W(\varphi, x)) d \varphi .
\end{aligned}
$$

Considerando que $f^{*}(x ; \alpha)$ e $f^{* \prime}(x ; \alpha)$ podem ser calculados como nas seções anteriores, agora é preciso somente calcular a integral que aparece no terceiro termo de (4.30). Representações alternativas da segunda derivada da densidade de uma distribuição estável padrão no caso simétrico nos casos extremos devem ser utilizadas.

Para $x \rightarrow 0$ e $0<\alpha \leq 2, \alpha \neq 1$, diferenciando-se (4.28), tem-se:

$$
f^{* \prime \prime}(x ; \alpha)=\frac{1}{\pi \alpha} \sum_{k=1}^{\infty} \frac{\Gamma((2 k+1) / \alpha)}{(2 k-2) !}(-1)^{k} x^{2 k-2} .
$$

De maneira similar, para $x \rightarrow \infty$ e $0<\alpha \leq 2, \alpha \neq 1$, tem-se

$$
f^{* \prime \prime}(x ; \alpha)=\frac{1}{\pi} \sum_{k=1}^{\infty} \frac{\Gamma(k \alpha+3)}{k !}(-1)^{k-1} \operatorname{sen}\left(\frac{\pi \alpha k}{2}\right) x^{-k \alpha-3} .
$$

A Tabela 4.3, baseada em Matsui e Takemura (2006), resume o uso de várias expansões. Novamente, aqui considera-se $x<0,6$ para se utilizar a expansão no caso de $x \rightarrow 0$, diferente de Matsui e Takemura (2006), que consideram $x<10^{-3}$.

O programa STABLE, desenvolvido pela Robust Analysis Inc para Matlab, Excel, R e Mathematica, é o mais eficaz para efetuar cálculos envolvendo distribuições estáveis, incluindo função densidade e suas primeira e segunda derivadas. Após diversos testes, foi verificado que a obtenção de tais quantidades através dos cálculos apresentados neste Capítulo é mais eficiente, em termos de 
tempo computacional, em comparação com o programa STABLE, com semelhante grau de precisão, para $1<\alpha \leq 2$.

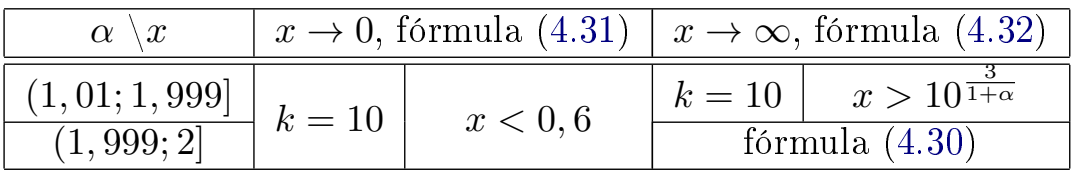

Tabela 4.3: Aproximações para $f^{* \prime \prime}(x ; \alpha)$ em casos extremos, $1,01<\alpha \leq 2$

\subsubsection{Terceira derivada em relação ao parâmetro de escala}

O uso de $f^{* \prime \prime \prime}(x)$ em geral não é necessário quando se trabalha com distribuições estáveis. Logo, seu cálculo não está disponível na literatura. Neste trabalho, a terceira derivada é necessária no processo de estimação do modelo proposto no Capítulo 5. Tal situação ocorre porque a função score, que envolve a primeira derivada, faz parte da estrutura do modelo GAS, e ao se realizar duas diferenciações para a obtenção da matriz Hessiana, a terceira derivada aparece.

Diferenciando-se (4.30), tem-se

$$
\begin{aligned}
f^{* \prime \prime \prime}(x)= & -\frac{1}{x^{3}} \frac{1}{(\alpha-1)^{2}} f^{*}(x ; \alpha)+\frac{\left(-2 \alpha^{2}+8 \alpha-7\right)}{x^{2}(\alpha-1)^{2}} f^{* \prime}(x ; \alpha)+\frac{(6-4 \alpha)}{x(\alpha-1)} f^{* \prime \prime}(x ; \alpha) \\
& -\frac{1}{x^{4}} \frac{\alpha^{4} \operatorname{sinal}(\alpha-1)}{\pi(\alpha-1)^{4}} \int_{0}^{\frac{\pi}{2}} W^{2}(\varphi, x)\left(\begin{array}{c}
2-4 W(\varphi, x)+ \\
+W^{2}(\varphi, x) \exp (-W(\varphi, x))
\end{array}\right) d \varphi .
\end{aligned}
$$

Como $f^{*}(x ; \alpha), f^{* \prime}(x ; \alpha)$ e $f^{* \prime \prime}(x ; \alpha)$ podem ser calculados de acordo com as seções anteriores, agora é preciso somente calcular a integral que aparece na quarta parcela de (4.33). Representações alternativas da segunda derivada da densidade de uma distribuição estável padrão no caso simétrico nos extremos devem ser utilizadas.

Para $x \rightarrow 0$ e $0<\alpha \leq 2, \alpha \neq 1$, diferenciando-se (4.31), tem-se:

$$
f^{* \prime \prime \prime}(x ; \alpha)=\frac{1}{\pi \alpha} \sum_{k=1}^{\infty} \frac{\Gamma((2 k+3) / \alpha)}{(2 k-1) !}(-1)^{k+1} x^{2 k-1} .
$$

De forma similar, para $x \rightarrow \infty$ e $0<\alpha \leq 2, \alpha \neq 1$, tem-se:

$$
f^{* \prime \prime \prime}(x ; \alpha)=\frac{1}{\pi} \sum_{k=1}^{\infty} \frac{\Gamma(k \alpha+4)}{k !}(-1)^{k} \operatorname{sen}\left(\frac{\pi \alpha k}{2}\right) x^{-k \alpha-4} .
$$

A Tabela 4.4 é similar às Tabelas das Subseções anteriores. Ela sumariza as análises nos casos

\begin{tabular}{|c|c|c|c|c|}
\hline$\alpha \backslash x$ & \multicolumn{2}{|c|}{$x \rightarrow 0$, fórmula $(4.34)$} & \multicolumn{2}{|c|}{$x \rightarrow \infty$, fórmula $(4.35)$} \\
\hline$(1,01 ; 1,999]$ & \multirow{2}{*}{$k=10$} & \multirow{2}{*}{$x<0,6$} & $k=10$ & $x>10^{\frac{3}{1+\alpha}}$ \\
\hline$(1,999 ; 2]$ & & & \multicolumn{2}{|c|}{ fórmula (4.33) } \\
\hline
\end{tabular}
de fronteira. Tais análises foram conduzidas de forma similar às feitas para os casos da densidade e de suas primeira e segunda derivadas, com diversos testes feitos para avaliar a confiabilidade dos resultados.

Tabela 4.4: Aproximações para $f^{* \prime \prime \prime}(x ; \alpha)$ em casos extremos, $1,01<\alpha \leq 2$ 


\subsubsection{Primeira derivada com relação a $\alpha$}

Como nos casos anteriores, é necessário apenas investigar o caso padrão $(\delta=0$ e $\gamma=1)$. Apesar das representações de $f_{\alpha}^{*}(x ; \alpha)$ por vezes se tornarem complicadas, uma análise cuidadosa pode levar a cálculos precisos e eficientes. A diferenciação de (4.9) leva à seguinte representação:

$$
\begin{aligned}
f_{\alpha}^{*}(x ; \alpha)= & -\frac{1}{\alpha(\alpha-1)} f^{*}(x ; \alpha) \\
& +\frac{\alpha}{\pi(\alpha-1) x} \int_{0}^{\frac{\pi}{2}} W_{\alpha}(\varphi, x)(1-W(\varphi, x))(\exp (-W(\varphi, x)) d \varphi,
\end{aligned}
$$

em que

$$
\begin{aligned}
W_{\alpha}(\varphi, x) & =-W(\varphi, x)\left(h_{1}(\varphi, x)+h_{2}(\varphi, x)+h_{3}(\varphi, x)\right), \\
h_{1}(\varphi, x) & =\frac{1}{(\alpha-1)^{2}} \log \left(\frac{x \cos \varphi}{\operatorname{sen}(\alpha \varphi)}\right), \\
h_{2}(\varphi, x) & =\frac{\alpha \varphi}{\alpha-1} \frac{1}{\tan (\alpha \varphi)}, \\
h_{3}(\varphi, x) & =\varphi \tan ((\alpha-1) \varphi) .
\end{aligned}
$$

Como no caso da densidade, representações alternativas da primeira derivada da densidade em relação a $\alpha$ nos extremos devem ser utilizadas.

Para $x \rightarrow 0$ e $0<\alpha \leq 2, \alpha \neq 1$, diferenciando-se (4.23) com relação a $\alpha$, tem-se

$$
f_{\alpha}^{*}(x ; \alpha)=\frac{1}{\pi \alpha^{2}} \sum_{k=1}^{\infty} \frac{(-2 k-1) \Gamma^{\prime}((2 k+1) / \alpha) / \alpha-\Gamma((2 k+1) / \alpha)}{(2 k) !}(-1)^{k} x^{2 k} .
$$

De maneira similar, para $x \rightarrow \infty$ e $0<\alpha \leq 2, \alpha \neq 1$, diferenciando (4.24) com relação a $\alpha$, tem-se:

$$
f_{\alpha}^{*}(x ; \alpha)=\frac{1}{\pi} \sum_{k=1}^{\infty} \frac{(-1)^{k-1} x^{-k \alpha-1}}{k !}\left(\begin{array}{l}
\Gamma^{\prime}(k \alpha+1) k \operatorname{sen}\left(\frac{\pi \alpha k}{2}\right)+\Gamma(k \alpha+1) \cos \left(\frac{\pi \alpha k}{2}\right) \frac{\pi k}{2} \\
-\Gamma(k \alpha+1) \operatorname{sen}\left(\frac{\pi \alpha k}{2}\right) k \ln (x)
\end{array}\right) .
$$

A Tabela 4.5 traz um resumo das aproximações da primeira derivada da densidade com relação a $\alpha$. Assim como nos casos anteriores, há uma diferença em relação ao considerado por Matsui e Takemura (2006) no caso $\alpha \in(1,01 ; 1,9999]$ para $x \rightarrow 0$. Os autores consideram $x<10^{-5}$, mas após vários testes foi verificado que deve-se considerar $x<0,6$, conforme exposto na Tabela. Os outros valores também foram checados e estão de acordo com o que os autores obtiveram.

\begin{tabular}{|c|c|c|c|c|}
\hline$\alpha \backslash x$ & \multicolumn{2}{|c|}{$x \rightarrow 0$, fórmula (4.41) } & \multicolumn{2}{c|}{$x \rightarrow \infty$, fórmula (4.42) } \\
\hline \hline$(1,01 ; 1,9999]$ & $k=10$ & $x<0,6$ & $k=10$ & $x>10^{\frac{3}{1+\alpha}}$ \\
\hline$(1,9999 ; 2]$ & $k=85$ & $x \leq 8$ & $k=20$ & $x>8$ \\
\hline
\end{tabular}

Tabela 4.5: Aproximações para $f_{\alpha}^{*}(x ; \alpha)$ nos casos extremos, $1,01<\alpha \leq 2$ 


\subsubsection{Segunda derivada em relação a $\alpha$}

Diferenciando-se (4.36), a segunda derivada de $f^{*}(x ; \alpha)$ com relação a $\alpha$ é dada por

$$
\begin{aligned}
f_{\alpha \alpha}^{*}(x ; \alpha)= & \frac{2}{\alpha^{2}(\alpha-1)} f^{*}(x ; \alpha)-\frac{2}{\alpha(\alpha-1)} f_{\alpha}^{*}(x ; \alpha) \\
& +\frac{\alpha}{\pi(\alpha-1) x} \int_{0}^{\frac{\pi}{2}}\left(\begin{array}{l}
\left(\left(W_{\alpha \alpha}(\varphi, x)-W_{\alpha}(\varphi, x)^{2}\right)(1-W(\varphi, x))\right. \\
\left.-W_{\alpha}(\varphi, x)\left(1-W_{\alpha}(\varphi, x)\right)\right) \exp (-W(\varphi, x))
\end{array}\right) d \varphi,
\end{aligned}
$$

em que

$$
\begin{aligned}
W_{\alpha \alpha}(\varphi, x) & =-W_{\alpha}(\varphi, x)\left(h_{1}(\varphi, x)+h_{2}(\varphi, x)+h_{3}(\varphi, x)\right. \\
& -W(\varphi, x)\left(h_{1, \alpha}(\varphi, x)+h_{2, \alpha}(\varphi, x)+h_{3, \alpha}(\varphi, x)\right), \\
h_{1, \alpha}(\varphi, x) & =-\frac{2}{(\alpha-1)^{3}} \log \left(\frac{x \cos \varphi}{\operatorname{sen}(\alpha \varphi)}\right)-\frac{1}{(\alpha-1)^{2}} \frac{\varphi}{\tan (\alpha \varphi)}, \\
h_{2, \alpha}(\varphi, x) & =-\frac{\varphi}{(\alpha-1)^{2} \tan (\alpha \varphi)}-\frac{\alpha \varphi^{2}}{(\alpha-1) \operatorname{sen}^{2}(\alpha \varphi)}, \\
h_{3, \alpha}(\varphi, x) & =\frac{\varphi^{2}}{\cos ^{2}((\alpha-1) \varphi)} .
\end{aligned}
$$

Representações alternativas da segunda derivada da densidade de uma distribuição estável padrão no caso simétrico em relação a $\alpha$ nos extremos devem ser utilizadas.

Para $x \rightarrow 0$ e $0<\alpha \leq 2, \alpha \neq 1$, diferenciando-se (4.41) com relação a $\alpha$, tem-se:

$$
f_{\alpha \alpha}^{*}(x ; \alpha)=\frac{1}{\pi \alpha^{2}} \sum_{k=1}^{\infty} \frac{(-1)^{k} x^{2 k}}{(2 k) !}\left(\begin{array}{l}
2 \Gamma((2 k+1) / \alpha) / \alpha-4(-2 k-1) \Gamma^{\prime}((2 k+1) / \alpha) \alpha^{2} \\
-(-2 k-1)^{2} \Gamma^{\prime \prime}((2 k+1) / \alpha) / \alpha^{3}
\end{array}\right) .
$$

De maneira similar, para $x \rightarrow \infty$ e $0<\alpha \leq 2, \alpha \neq 1$, diferenciando (4.42) com relação a $\alpha$, tem-se

$$
\begin{aligned}
f_{\alpha \alpha}^{*}(x ; \alpha) & =\frac{1}{\pi} \sum_{k=1}^{\infty} \frac{(-1)^{k-1} x^{-k \alpha-1}}{k !} \\
& \times\left(\Gamma^{\prime \prime}(k \alpha+1) k^{2} \operatorname{sen}\left(\frac{\pi \alpha k}{2}\right)+\Gamma^{\prime}(k \alpha+1) k \cos \left(\frac{\pi \alpha k}{2}\right) \frac{\pi \alpha k}{2}\right. \\
& -\Gamma^{\prime}(k \alpha+1) k^{2} \operatorname{sen}\left(\frac{\pi \alpha k}{2}\right) \ln (x)+\Gamma^{\prime}(k \alpha+1) k \cos \left(\frac{\pi \alpha k}{2}\right) \frac{\pi \alpha k}{2} \\
& -\Gamma(k \alpha+1) \operatorname{sen}\left(\frac{\pi \alpha k}{2}\right)\left(\frac{\pi \alpha k}{2}\right)^{2}-\Gamma(k \alpha+1) \operatorname{sen}\left(\frac{\pi \alpha k}{2}\right) k \ln (x) \\
& -\Gamma^{\prime}(k \alpha+1) k^{2} \operatorname{sen}\left(\frac{\pi \alpha k}{2}\right) \ln (x)-\Gamma(k \alpha+1) \cos \left(\frac{\pi \alpha k}{2}\right) \frac{\pi \alpha k}{2} k \ln (x) \\
& \left.+\Gamma(k \alpha+1) \operatorname{sen}\left(\frac{\pi \alpha k}{2}\right)(k \ln (x))^{2}\right) .
\end{aligned}
$$

A Tabela 4.6 é um resumo das aproximações da segunda derivada da densidade com relação a $\alpha$, também com $x<0,6$ considerado para $x \rightarrow 0$ se $\alpha \in(1,01 ; 1,9999]$, ao invés de $x<10^{-3}$ como considerado por Matsui e Takemura (2006).

\begin{tabular}{|c|c|c|c|c|}
\hline$\alpha \backslash x$ & \multicolumn{2}{|c|}{$x \rightarrow 0$, fórmula (4.49) } & \multicolumn{2}{c|}{$x \rightarrow \infty$, fórmula (4.50) } \\
\hline \hline$(1,01 ; 1,9999]$ & $k=10$ & $x<0,6$ & $k=10$ & $x>10^{\frac{3}{1+\alpha}}$ \\
\hline$(1,9999 ; 2]$ & $k=85$ & $x \leq 8$ & $k=20$ & $x>8$ \\
\hline
\end{tabular}

Tabela 4.6: Aproximações para $f_{\alpha \alpha}^{*}(x ; \alpha)$ nos casos extremos, $1,01<\alpha \leq 2$ 


\subsubsection{Segunda derivada com relação a $\alpha$ e $x$}

A segunda derivada da densidade em relação a $\alpha$ e $x, f_{\alpha x}^{*}(x ; \alpha)$, não foi desenvolvida por Matsui e Takemura (2006), mas será necessária no processo de estimação do modelo proposto no Capítulo 5 e pode ser obtida pelo mesmo procedimento das Sessões anteriores.

Diferenciando-se (4.36) com relação a $x$, tem-se

$$
\begin{aligned}
f_{\alpha x}^{*}(x ; \alpha)= & -\frac{f^{*}(x ; \alpha)}{\alpha(\alpha-1)}-\frac{1}{\alpha(\alpha-1)} f^{* \prime}(x ; \alpha)-\frac{1}{x} f_{\alpha}^{*}(x ; \alpha) \\
& +\frac{\alpha}{\pi(\alpha-1) x} \int_{0}^{\frac{\pi}{2}}\left(\begin{array}{l}
\left(\left(W_{\alpha x}(\varphi, x)-W_{\alpha}(\varphi, x) W_{x}(\varphi, x)\right)(1-W(\varphi, x))\right. \\
\left.-W_{\alpha}(\varphi, x) W_{x}(\varphi, x)\right) \exp (-W(\varphi, x))
\end{array}\right) d \varphi,
\end{aligned}
$$

em que

$$
\begin{aligned}
W_{x}(\varphi, x) & =\frac{\alpha}{(\alpha-1) x} W(\varphi, x), \\
W_{\alpha x}(\varphi, x) & =W_{x}(\varphi, x)\left(h_{1}(\varphi, x)+h_{2}(\varphi, x)+h_{3}(\varphi, x)\right)-W(\varphi, x) h_{1, x}(\varphi, x), \\
h_{1, x}(\varphi, x) & =\frac{1}{(\alpha-1)^{2} x} .
\end{aligned}
$$

Representações alternativas da segunda derivada da densidade de uma distribuição estável padrão no caso simétrico em relação a $\alpha$ e $x$ nos extremos devem ser utilizadas.

Para $x \rightarrow 0$ e $0<\alpha \leq 2, \alpha \neq 1$, diferenciando-se (4.41) com relação a $x$, tem-se

$$
f_{\alpha x}^{*}(x ; \alpha)=\frac{1}{\pi \alpha^{2}} \sum_{k=1}^{\infty} \frac{(-2 k-1) \Gamma^{\prime}((2 k+1) / \alpha) / \alpha-\Gamma((2 k+1) / \alpha)}{(2 k) !}(-1)^{k} 2 k x^{2 k-1} .
$$

Para $x \rightarrow \infty$ e $0<\alpha \leq 2, \alpha \neq 1$, diferenciando (4.42) com relação a $x$, tem-se

$$
\begin{aligned}
f_{\alpha x}^{*}(x ; \alpha) & =\frac{1}{\pi} \sum_{k=1}^{\infty} \frac{(-1)^{k-1} x^{-k \alpha-2}}{k !} \\
& \times\left(\Gamma^{\prime}(k \alpha+1) k \operatorname{sen}\left(\frac{\pi \alpha k}{2}\right)(-k \alpha-1)+\Gamma(k \alpha+1) \cos \left(\frac{\pi \alpha k}{2}\right) \frac{\pi \alpha k}{2}(-k \alpha-1)\right. \\
& \left.-\Gamma(k \alpha+1) \operatorname{sen}\left(\frac{\pi \alpha k}{2}\right) k-\Gamma(k \alpha+1) \operatorname{sen}\left(\frac{\pi \alpha k}{2}\right) \ln (x)(-k \alpha-1) k\right) .
\end{aligned}
$$

A Tabela 4.7 é um resumo das aproximações da segunda derivada da densidade com relação a $\alpha$ e $x$. Tais análises foram conduzidas baseadas em diversos testes para avaliar a confiabilidade dos resultados.

\begin{tabular}{|c|c|c|c|c|}
\hline$\alpha \backslash x$ & \multicolumn{2}{|c|}{$x \rightarrow 0$, fórmula (4.55) } & \multicolumn{2}{c|}{$x \rightarrow \infty$, fórmula (4.56) } \\
\hline \hline$(1,01 ; 1,9999]$ & $k=10$ & $x<0,6$ & $k=10$ & $x>10^{\frac{3}{1+\alpha}}$ \\
\hline$(1,9999 ; 2]$ & $k=85$ & $x \leq 8$ & $k=20$ & $x>8$ \\
\hline
\end{tabular}

Tabela 4.7: Aproximações para $f_{\alpha x}^{*}(x ; \alpha)$ nos casos extremos, $1,01<\alpha \leq 2$ 


\subsection{Informação de Fisher e suas derivadas}

Utilizando os resultados das seções anteriores, é possível calcular de maneira precisa a informação de Fisher de distribuições estáveis simétricas.

No modelo proposto no Capítulo 5, o interesse será na informação de Fisher com as derivadas calculadas em relação a $\sigma$. Como o parâmetro de escala da distribuição estável considerada é $\gamma(\sigma)$, a informação de Fisher é definida como

$$
\mathcal{I}_{\sigma}=\mathbb{E}\left[\nabla(x ; \gamma(\sigma), \alpha)^{2}\right]=\int l_{\sigma}(x ; \gamma(\sigma), \alpha)^{2} f(x ; \gamma(\sigma), \alpha) d x=\int \frac{f_{\sigma}(x ; \gamma(\sigma), \alpha)^{2}}{f(x ; \gamma(\sigma), \alpha)} d x .
$$

$\mathrm{O}$ valor de $\mathcal{I}_{\sigma}$, assim como de suas derivadas, obviamente dependem de $\sigma$, indicado pelo subescrito, e também do valor de $\alpha$. No caso de distribuição estável padrão, que será utilizada logo a seguir, em que o parâmetro de escala $\gamma=1$, a informação depende apenas de $\alpha$. No entanto, $\alpha$ é omitido da notação por simplicidade.

As derivadas de $\mathcal{I}_{\sigma}$ com relação a $\sigma$ serão necessárias para o procedimento de estimação do modelo que será proposto no Capítulo 5, e são obtidas por diferenciação de (4.57):

$$
\begin{aligned}
\frac{\partial \mathcal{I}_{\sigma}}{\partial \sigma}= & \int\left(2 \frac{f_{\sigma}(x ; \gamma(\sigma), \alpha) f_{\sigma \sigma}(x ; \gamma(\sigma), \alpha)}{f(x ; \gamma(\sigma), \alpha)}-\frac{f_{\sigma}(x ; \gamma(\sigma), \alpha)^{3}}{f(x ; \gamma(\sigma), \alpha)^{2}}\right) d x, \\
\frac{\partial \mathcal{I}_{\sigma}^{2}}{\partial^{2} \sigma}=\int & \left(2 \frac{f_{\sigma}(x ; \gamma(\sigma), \alpha)+f_{\sigma \sigma}(x ; \gamma(\sigma), \alpha)^{2}+f_{\sigma \sigma \sigma}(x ; \gamma(\sigma), \alpha)}{f(x ; \gamma(\sigma), \alpha)}\right. \\
& \left.-5 \frac{f_{\sigma}(x ; \gamma(\sigma), \alpha)^{2} f_{\sigma \sigma}(x ; \gamma(\sigma), \alpha)}{f(x ; \gamma(\sigma), \alpha)^{2}}+2 \frac{f_{\sigma}(x ; \gamma(\sigma), \alpha)^{4}}{f_{\sigma \sigma \sigma}(x ; \gamma(\sigma), \alpha)}\right) d x .
\end{aligned}
$$

Nas Seções anteriores, foi mostrado como calcular a densidade e suas derivadas com relação a $\sigma$. Para os cálculos das integrais em (4.57), (4.58) e (4.59), algum método numérico deve ser utilizado.

Mas há uma maneira mais simples de fazê-lo.

Pode-se obter o valor de $\mathcal{I}_{\sigma}$ em função do valor da informação no caso padrão com respeito ao parâmetro de escala, digamos $\mathcal{I}^{\text {std }}$. Nesse caso, a integral em si não dependeria do valor de $\sigma$, tornando os cálculos menos custosos, principalmente em um modelo em que o parâmetro de escala é variável. Substituindo $f_{\sigma}(x ; \gamma(\sigma), \alpha)^{2}$ e $f(x ; \gamma(\sigma), \alpha)$ pelas expressões de (4.1) e (4.3) em (4.57), temos

$$
\begin{aligned}
\mathcal{I}_{\sigma} & =\int \frac{\gamma(\sigma)}{f^{*}\left(\frac{x}{\gamma(\sigma)} ; \alpha\right)}\left[-\frac{\gamma^{\prime}(\sigma)}{\gamma(\sigma)^{2}} f^{*}\left(\frac{x}{\gamma(\sigma)} ; \alpha\right)-x \frac{\gamma^{\prime}(\sigma)}{\gamma(\sigma)^{3}} f^{* \prime}\left(\frac{x}{\gamma(\sigma)} ; \alpha\right)\right]^{2} d x \\
& =\frac{\gamma^{\prime}(\sigma)^{2}}{\gamma(\sigma)^{3}} \int \frac{1}{f^{*}\left(\frac{x}{\gamma(\sigma)} ; \alpha\right)}\left[-f^{*}\left(\frac{x}{\gamma(\sigma)} ; \alpha\right)-\frac{x}{\gamma(\sigma)} f^{* \prime}\left(\frac{x}{\gamma(\sigma)} ; \alpha\right)\right]^{2} d x \\
& =\frac{\gamma^{\prime}(\sigma)^{2}}{\gamma(\sigma)^{2}} \int \frac{\left(-f^{*}(x ; \alpha)-x f^{* \prime}(x ; \alpha)\right)^{2}}{f^{*}(x ; \alpha)} d x \\
& =\frac{\gamma^{\prime}(\sigma)^{2}}{\gamma(\sigma)^{2}} \mathcal{I}^{\text {std }} .
\end{aligned}
$$

No modelo proposto no Capítulo 5 , será considerado $\gamma(\sigma)=\sqrt{\sigma / 2}$, de modo que, para $\alpha=2$, a distribuição estável em questão seja a distribuição normal de variância $\sigma$. Nesse caso,

$$
\mathcal{I}_{\sigma}^{-1}=\frac{4 \sigma^{2}}{\mathcal{I}^{s t d}}
$$


e

$$
\begin{gathered}
\frac{\partial \mathcal{I}_{\sigma}^{-1}}{\partial \sigma}=\frac{8 \sigma}{\mathcal{I}^{s t d}}, \\
\frac{\partial^{2} \mathcal{I}_{\sigma}^{-1}}{\partial \sigma^{2}}=\frac{8}{\mathcal{I}^{s t d}} .
\end{gathered}
$$

Então, é preciso apenas de $\mathcal{I}^{\text {std }}$ para que se obtenham a informação de Fisher no caso não-padrão e suas primeira e segunda derivadas. 


\section{Capítulo 5}

\section{Modelo proposto}

O modelo proposto neste Capítulo é de uma classe de modelos de séries temporais não-lineares elaborados para extrair o sinal dinâmico de observações ruidosas. Tal sinal é uma medida de escala, a qual tem importância fundamental em séries temporais financeiras, nas quais volatilidade em conglomerados é algo corrente. Modelos autorregressivos de heteroscedasticidade condicional (GARCH) são usados amplamente para estimar a variância dinâmica em séries financeiras. No entanto, utilizar a variância como medida de escala pode não ser apropriado para distribuições condicionais não-gaussianas. Tal fato deve ser considerado, pois outro aspecto conhecido de retornos financeiros é que eles são caracterizados por caudas pesadas.

Equações dinâmicas de modelos GARCH são filtros. Assim como filtros para modelos de localização gaussianos são combinações lineares de observações passadas, os filtros GARCH também são. Devido a suas origens gaussianas, tratam-se usualmente de combinações lineares de observações passadas ao quadrado. Os modelos descritos aqui substituem as observações ou o quadrado delas pelo score da distribuição condicional.

A ideia de utilizar o score para guiar as dinâmicas em modelos não-gaussianos não é nova, mas até 2008 não tinha fundamentação teórica estabelecida. Naquele ano, Harvey e Chakravarty escreveram um artigo sobre modelos EGARCH. Ao mesmo tempo, Creal, Koopman e Lucas desenvolveram, de forma independente, uma classe de modelos guiados pelo score e também escreveram um artigo em 2008.

Harvey e Chakravarty utilizam o termo DCS (Dynamic Conditional Score), enquanto Creal, Koopman e Lucas utilizam GAS (Generalized Autoregressive Score). Harvey defende que, apesar do termo ser atrativo, a palavra "autorregressivo" parece se referir a uma estrutura dinâmica mais limitada do que é realmente o caso (Harvey, 2013).

Modelos GARCH são capazes de capturar dois aspectos importantes que caracterizam séries temporais de retornos financeiros: agrupamentos de volatilidades ao longo do tempo e excesso de leptocurtose devido a caudas pesadas, ou seja, as caudas da distribuição não-condicional são mais pesadas que as da distribuição normal. Ambos os fenômenos foram observados por Mandelbrot (1963). No entanto, em seu trabalho, ele focou na segunda propriedade e propôs o uso de uma distribuição estável. Tal sugestão foi corroborada por Fama (1963) e Fama (1965) logo em seguida. de Vries (1991) mostrou que não somente a distribuição não-condicional de retornos financeiros pode ser estável, mas, sob certas condições, a distribuição condicional de processos GARCH também é estável.

Ao ajustar modelos GARCH para séries temporais, frequentemente são observados resíduos com caudas pesadas. Para lidar com esse fato, modelos GARCH com distribuições inovacionais de caudas mais pesadas que as da normal aparecem na literatura. Entre eles, o modelo com distribuição t-Student (Bollerslev, 1987) e o modelo com a GED (distribuição generalizada de erros) (Nelson, 1991). Para permitir casos particulares de distribuições de retornos com caudas pesadas, modelos que consideram distribuições estáveis podem ser considerados.

No entanto, usualmente as distribuições estáveis são utilizadas para modelar a distribuição nãocondicional de retornos financeiros, e não a distribuição condicional. Isso ocorre principalmente 
pelas dificuldades práticas em implementar modelos estáveis, principalmente no que se refere a estimação de parâmetros (Mittnik et al., 1998). Mas há alguns modelos, com destaque para os da classe GARCH, que consideram distribuições condicionais estáveis (ver Panorska et al. (1995) e Mittnik et al. (2002)).

A classe de distribuições estáveis contém a distribuição normal como caso particular, mas também permite caudas pesadas, ou seja, variância infinita, e assimetria. Nem a distribuição $t$-Student nem a GED têm a última propriedade. A distribuição estável também possui uma propriedade de muito apelo: é a única distribuição que resulta de uma distribuição limite de somas de variáveis aleatórias i.i.d., conforme detalhado na Seção 3.5. Tal fato é adequado quando os termos de erro são por suposição a soma de efeitos externos que não são capturados pelo modelo.

Uma suposição importante de modelos GARCH usuais é que a distribuição condicional do processo possui segundo momento, impondo limites para o peso das caudas de sua distribuição nãocondicional. Dado que um grande número de dados financeiros exibem caudas substancialmente pesadas, essa suposição representa uma grande limitação dos modelos GARCH em análise de séries temporais financeiras. A distribuição $t$-Student permite caudas mais pesadas que as da distribuição normal, mas possui segundo momento finito. Mandelbrot (1963) destaca que, em certos conjuntos de dados, valores observados trazem evidência de esperança finita, mas variância infinita, característica com a qual a distribuição $t$-Student não consegue lidar adequadamente.

Além disso, a distribuição $t$-Student não possui a desejável propriedade de estabilidade. No caso de distribuições estáveis, a distribuição da soma de variáveis aleatórias i.i.d. também é estável e tem a mesma forma da distribuição dos somandos individuais (para mais detalhes, ver Seção 3.2). Tal propriedade é desejável por dois motivos:

- em séries financeiras, variações de preço em um dado intervalo de tempo podem ser consideradas somas de variações entre transações durante o intervalo. Se as variações entre transações são i.i.d. e têm distribuição estável, as variações do preço em um dia, uma semana, um mês ou em um intervalo particular também terão distribuição estável. Por exemplo, se a distribuição das variações de preço diário é estável com parâmetro de localização $\delta$ e parâmetro de escala $\gamma$, então a distribuição das variações em uma semana (5 dias úteis) também será estável com parâmetro de localização $5 \delta$ e parâmetro de escala $5 \gamma$. A distribuição normal tem essa propriedade, pois é um caso particular de distribuição estável, mas a t-Student não. É conveniente considerar uma distribuição cuja forma seja independente dos intervalos para os quais as variações de preços sejam calculadas (Fama, 1963); e

- a propriedade de estabilidade faz com que as distribuições estáveis sejam as únicas distribuições limites possíveis para somas de variáveis aleatórias i.i.d. Ou seja, as distribuições estáveis possuem domínios de atração (ver mais detalhes na Seção 3.5), o que fornece aproximações muito boas para várias classes de distribuições. No caso de retornos financeiros, mesmo que eles não sigam exatamente uma distribuição estável, uma distribuição estável será uma aproximação para sua distribuição.

As aplicações são bem sucedidas quando os dados apresentam caudas pesadas e são leptocúrticos. A Figura 5.1 traz comparações entre as densidades de distribuições estável simétrica $(\alpha=1,5$, $\beta=0, \delta=0$ e $\gamma=1 / \sqrt{2}$ ), normal e $t$-Student ( 7 graus de liberdade). As caudas da distribuição estável são obviamente mais pesadas que as da normal. Com relação à distribuição $t$-Student, a distribuição estável exibe maior concentração no centro da distribuição (ao redor de zero), por isso tendo um caráter ainda mais leptocúrtico que a $t$-Student.

Em dados financeiros, de acordo com Frain (2009), valores de $\alpha$ entre 1,5 e 1,8 geralmente são apropriados.

Por outro lado, modelos dinâmicos com parâmetros da distribuição de probabilidade dos dados variam no tempo, guiados por observações baseados no score da função preditiva de verossimilhança, foram utilizados com sucesso por diferentes autores em vários estudos empíricos. Há extensões para distribuições assimétricas e de caudas pesadas, assim como diferentes modelos dinâmicos de localização e escala e modelos dinâmicos de cópulas (Blasques et al., 2015). 


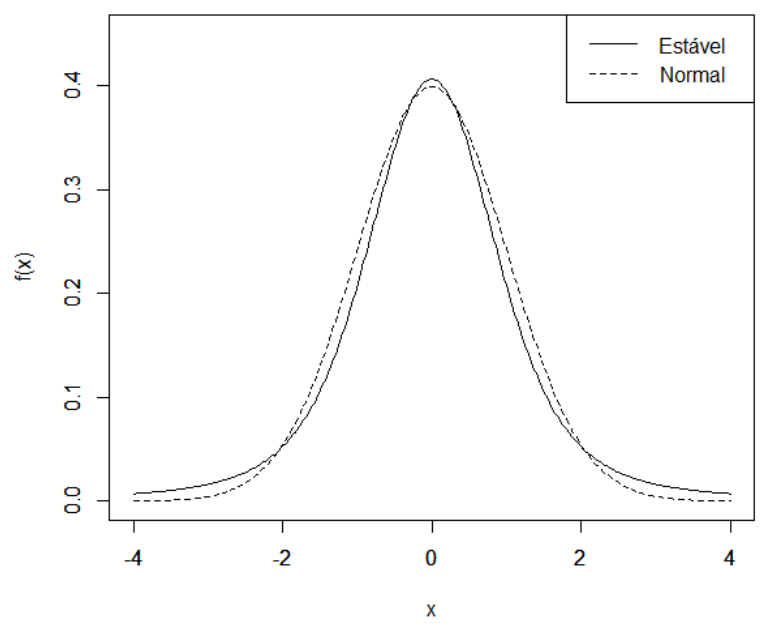

(a)

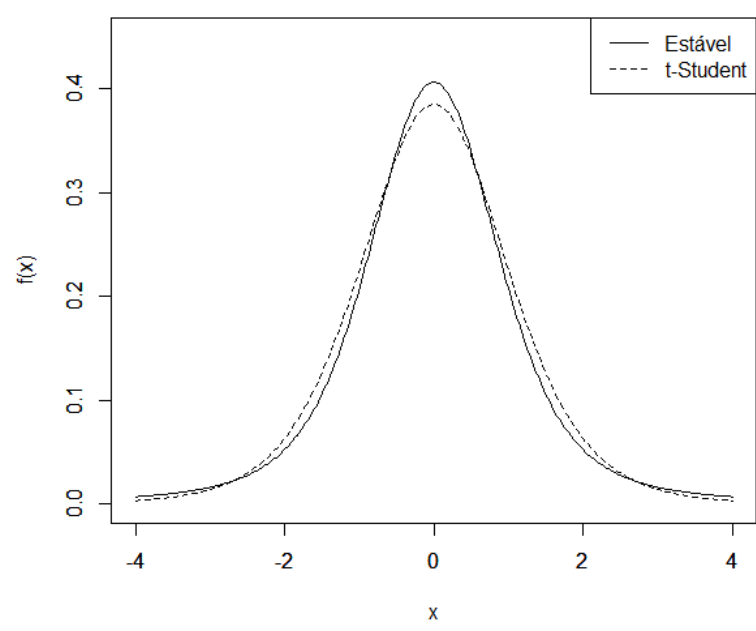

(b)

Figura 5.1: Funções densidade de probabilidade: distribuições estável $(\alpha=1,5)$, normal padrão e t-Student (7 graus de liberdade)

Usar o score na equação de evolução do parâmetro que varia no tempo da distribuição de probabilidade dos dados tem a vantagem de definir a direção de subida mais acentuada para melhorar o ajuste local do modelo em termos da verossimilhança no instante $t$ dado o valor calculado de tal parâmetro. Isso fornece uma direção natural para a evolução do parâmetro. Além disso, se a inversa da informação é escolhida como fator de ponderação, o mecanismo de evolução pode ser interpretado como um algoritmo de Newton-Raphson de estimação do parâmetro ao longo do tempo (Creal et al., 2011)

Além disso, o score depende da densidade completa, e não apenas do primeiro ou do segundo momento das observações. Ao explorar toda a estrutura da densidade, o modelo GAS introduz novas transformações aos dados que podem ser usados para a evolução do parâmetro variante no tempo (Creal et al., 2013).

Modelos GAS aplicados a dados de caudas pesadas têm sido muito estudados recentemente, pois tal comportamento é encontrado com frequência em séries financeiras. Algumas referências: Creal et al. (2011), Harvey e Luati (2014) e Janus et al. (2014).

No entanto, não há resultados na literatura ligando modelos GAS e distribuições estáveis. Em alguns casos, tais modelos podem descrever os parâmetros dinâmicos de maneira mais realista e representar o comportamento dos dados de maneira mais adequada, aproveitando-se das vantagens já citadas de cada uma das abordagens. O modelo proposto será apresentado na Seção 5.2.

\subsection{Modelos de volatilidade para dados com caudas pesadas}

De acordo com Morettin (2011), séries econômicas e financeiras apresentam algumas características particulares, sendo duas notáveis: heteroscedasticidade condicional (volatilidade) e a distribuição do retornos financeiros em geral apresenta caudas mais pesadas do que uma distribuição normal; além disso, a distribuição, embora aproximadamente simétrica, é leptocúrtica.

Nesta Seção, serão expostos três modelos disponíveis na literatura utilizados para modelar volatilidade quando os dados possuem caudas pesadas: GARCH com distribuição $t$-Student, GARCH com distribuição estável e GAS com distribuição $t$-Student. Serão aproveitados aspectos atraentes de cada modelo na proposição no novo modelo na Seção 5.2. 


\subsubsection{Modelos GARCH clássicos}

Modelos GARCH (Generalized Autoregressive Conditional Heteroscedasticity) foram introduzidos por Bollerslev (1986) como uma generalização dos modelos ARCH de Engle (1982). Em tais modelos, o retorno é não correlacionado serialmente, mas a volatilidade (variância condicional) depende de retornos passados por meio de uma função quadrática. A vantagem do modelo GARCH é que pode ser usado para descrever a volatilidade com menos parâmetros que um modelo ARCH.

$\mathrm{Um}$ modelo $\operatorname{GARCH}(p, q)$ é definido por

$$
\begin{aligned}
y_{t} & =\sqrt{\sigma_{t}} \epsilon_{t}, \\
\sigma_{t} & =\omega+\sum_{i=1}^{p} A_{i} y_{t-i}^{2}+\sum_{j=1}^{q} B_{j} \sigma_{t-j},
\end{aligned}
$$

em que $\epsilon_{t}$ são variáveis aleatórias i.i.d. com média zero, $\omega>0, A_{i} \geq 0, i=1, \ldots, p-1, B_{j} \geq 0$, $j=1, \ldots, q-1, A_{p}>0, B_{q}>0, \sum_{i=1}^{m}\left(A_{i}+B_{i}\right)<1, m=\max (p, q)$.

Coeficientes positivos são uma condição suficiente, mas não necessária, para que $\sigma_{t}>0$, Para condições gerais, ver Nelson e Cao (1992).

A condição $\sum_{i=1}^{m}\left(A_{i}+B_{i}\right)<1$ é suficiente e necessária para que o processo seja estacionário de segunda ordem (Bollerslev, 1986). Bougerol e Picard (1992) derivaram condições suficientes e necessárias para estacionariedade estrita.

Para previsão da volatilidade, se $T$ é o tamanho da amostra para a qual o modelo é ajustado, então o valor da previsão $h$ passos à frente de $T$, denotada por $\hat{\sigma}_{T+h}$, é dada por

$$
\hat{\sigma}_{T+h}=\hat{\omega}+\sum_{i=1}^{p} \hat{A}_{i} \hat{y}_{T+h-i}^{2}+\sum_{j=1}^{q} \hat{B}_{j} \hat{\sigma}_{T+h-j},
$$

em que $\hat{\omega}, \hat{A}_{i}, i=1, \ldots, p, \hat{B}_{j}, j=1, \ldots, q$ são os valores estimados dos parâmetros do modelo. Se $T+h-k \leq T$, então $\hat{\sigma}_{T+h-k}=\sigma_{T+h-k}$ e $\hat{y}_{T+h-k}^{2}=y_{T+h-k}^{2}$. Caso contrário, $\hat{y}_{T+h-k}^{2}$ é obtido pela esperança condicional de $y_{T+h-k}^{2}$, que é igual a $\hat{\sigma}_{T+h-k}$.

Em modelos GARCH, volatilidades altas são precedidas de retornos ou volatilidades grandes, observando-se grupos de volatilidades presentes em séries financeiras.

Uma primeira escolha para a distribuição de $\epsilon_{t}$ é a normal. No entanto, um fato conhecido de dados financeiros é que estes usualmente possuem caudas pesadas. Bollerslev (1987) utilizou pela primeira vez uma distribuição $t$-Student padronizada, ou seja, com variância unitária. Tal modelo é frequentemente utilizado desde então quando os dados exibem caudas mais pesadas que as da distribuição normal.

A identificação da ordem de um modelo GARCH a ser ajustado a uma série real usualmente é difícil. Morettin (2011) recomenda o uso de modelos de ordens baixas, como $(1,1),(1,2),(2,1)$ ou $(2,2)$, e depois que se escolha o modelo com base em alguns critérios, como AIC, BIC, logverossimilhança, análise de resíduos e alguma função perda. Zivot (2009) destaca que, na prática, o modelo com ordens $p=1$ e $q=1$ é o mais frequente e dificilmente é superado por outros modelos GARCH.

Daqui para frente, o modelo GARCH com distribuição condicional normal será referido como GARCH gaussiano, e o modelo GARCH com distribuição condicional $t$-Student será referido como GARCH $t$-Student.

Para mais detalhes acerca das propriedades do modelo GARCH, ver Bollerslev (1986), Bollerslev (1987) e Morettin (2011).

\subsubsection{Modelos GARCH com distribuições estáveis}

Panorska et al. (1995) introduziram os modelos GARCH com distribuição condicional estável para lidar com séries temporais de caudas pesadas. 
A sequência $y_{t}$ segue um processo $\operatorname{GARCH}_{\alpha, \beta}(p, q)$ estável (corrigido pela média) se

$$
\begin{aligned}
y_{t} & =\sigma_{t} \epsilon_{t}, \quad \epsilon_{t} \sim S_{\alpha}(\beta, 0,1), \\
\sigma_{t} & =\omega+\sum_{i=1}^{p} A_{i}\left|y_{t-i}\right|+\sum_{j=1}^{q} B_{j} \sigma_{t-j},
\end{aligned}
$$

em que $\omega>0, A_{i} \geq 0, i=1, \ldots, p-1, B_{j} \geq 0, j=1, \ldots, q-1, A_{p}>0, B_{q}>0$ e $S_{\alpha}(\beta, 0,1)$ denota a distribuição estável padrão (não necessariamente simétrica).

Os autores assumem que $1<\alpha \leq 2$, ou seja, a média da distribuição estável existe. Tal suposição não é muito restritiva no contexto de modelagem financeira, já que a maioria das séries temporais financeiras de interesse parece contar com primeiro momento finito.

Existe uma diferença notável entre esse modelo e o modelo GARCH clássico, pois a dependência temporal da volatilidade não é mais representada por uma relação quadrática através de $y_{t}^{2}$. Tal relação não é considerada devido ao fato da distribuição estável não necessariamente possuir segundo momento, e utilizar o quadrado das observações na equação de $\sigma_{t}$ levaria a dificuldades no tratamento do modelo. Ou seja, mesmo se $\alpha=2$ for considerado, o que implica em uma distribuição normal, o modelo GARCH gaussiano não é um caso particular do modelo GARCH com distribuição estável.

No mesmo artigo, os autores provam que a condição de estacionariedade estrita do modelo é

$$
\omega<0 \text { e } \quad \lambda_{\alpha} \sum_{i=1}^{p} A_{i}+\sum_{j=1}^{q} B_{j}<1,
$$

em que

$$
\lambda_{\alpha}=\mathbb{E}\left[\left|\epsilon_{1}\right|\right]=\Gamma\left(1-\frac{1}{\alpha}\right) \int_{0}^{\infty} u^{-2} \operatorname{sen}^{2} u d u .
$$

Para previsão da volatilidade em um modelo simétrico $(\beta=0)$, se $T$ é o tamanho da amostra para a qual o modelo é ajustado, então o valor da previsão $h$ passos à frente de $T$, denotada por $\hat{\sigma}_{T+h}$, é dada por

$$
\hat{\sigma}_{T+h}=\hat{\omega}+\sum_{i=1}^{p} \hat{A}_{i}\left|\hat{y}_{T+h-i}\right|+\sum_{j=1}^{q} \hat{B}_{j} \hat{\sigma}_{T+h-j},
$$

em que $\hat{\omega}, \hat{A}_{i}, i=1, \ldots, p, \hat{B}_{j}, j=1, \ldots, q$ são os valores estimados dos parâmetros do modelo. Se $T+h-k \leq T$, então $\hat{\sigma}_{T+h-k}=\sigma_{T+h-k}$ e $\left|\hat{y}_{T+h-k}\right|=\left|y_{T+h-k}\right|$. Caso contrário, $\left|\hat{y}_{T+h-k}\right|$ é obtido pela esperança condicional de $\left|y_{T+h-k}\right|$, que é igual a $\frac{2}{\pi} \Gamma(1-1 / \hat{\alpha}) \hat{\sigma}_{T+h-k}$, em que $\hat{\alpha}$ é a estimativa de $\alpha$ obtida pelo modelo.

Daqui para frente, o modelo GARCH com distribuição condicional estável será referido como GARCH estável. Nas aplicações do Capítulo 6, será considerado $\beta=0$ (distribuição simétrica), para fins de comparação com os outros modelos, que também são simétricos.

Mittnik et al. (2002) propuseram uma classe mais geral chamada de stable-power-GARCH, em que a dependência temporal da variância condicional segue uma relação de potência da ordem de $\delta$, em que $1<\delta<\alpha$. O modelo GARCH gaussiano também não é um caso particular deste. Tal modelo não será considerado neste trabalho. 


\subsubsection{Modelos GAS com distribuição $t$-Student}

Vamos definir nesta Seção um modelo $\operatorname{GAS}(p, q)$, já explorado no Capítulo 2, para modelagem da variância condicional. Tal modelo é estruturado por

$$
\begin{aligned}
y_{t} & =\sqrt{\sigma_{t}} \epsilon_{t}, \\
\sigma_{t} & =\omega+\sum_{i=1}^{p} A_{i} s_{t-i}+\sum_{j=1}^{q} B_{j} \sigma_{t-j},
\end{aligned}
$$

em que $\epsilon_{t}$ tem distribuição $t$-Student padronizada (variância unitária) com $\nu$ graus de liberdade.

Nota-se que a estrutura é muito parecida com a do modelo $\operatorname{GARCH}(p, q)$. A diferença fica por conta da utilização da função $s_{t}$ ao invés de $y_{t}^{2}$ na equação de evolução de $\sigma_{t}$. Tal função é definida por

$$
s_{t}=S_{t} \nabla_{t}
$$

em que

$$
\nabla_{t}=\frac{\partial \ln f\left(y_{t} ; \sigma_{t}\right)}{\partial \sigma_{t}}=\frac{\partial \ln f_{t}}{\partial \sigma_{t}}
$$

é o score calculado em $\sigma_{t}$ e $S_{t}$ é uma função de ponderação, escolhida aqui como $S_{t}=\mathcal{I}_{t}^{-1}=$ $\mathbb{E}_{t}\left[\nabla_{t}^{2}\right]^{-1}$, com $f\left(y_{t} ; \sigma_{t}\right)=f_{t}$ sendo a densidade de $y_{t}$. Daqui para frente, tal modelo será referido como GAS $t$-Student.

Para previsão da volatilidade, se $T$ é o tamanho da amostra para a qual o modelo é ajustado, então o valor da previsão $h$ passos à frente de $T$, denotada por $\hat{\sigma}_{T+h}$, é dada por

$$
\hat{\sigma}_{T+h}=\hat{\omega}+\sum_{i=1}^{p} \hat{A}_{i} \hat{s}_{T+h-i}+\sum_{j=1}^{q} \hat{B}_{j} \hat{\sigma}_{T+h-j},
$$

em que $\hat{\omega}, \hat{A}_{i}, i=1, \ldots, p, \hat{B}_{j}, j=1, \ldots, q$ são os valores estimados dos parâmetros do modelo. Se $T+h-k \leq T$, então $\hat{\sigma}_{T+h-k}=\sigma_{T+h-k}$ e $\hat{s}_{T+h-k}=s\left(\hat{y}_{T+h-k}\right)=s\left(y_{T+h-k}\right)$. Caso contrário, $\hat{s}_{T+h-k}$ é obtido pela esperança condicional de $s_{T+h-k}=s\left(y_{T+h-k}\right)$, que, pelas propriedades do score, é zero.

Conforme já destacado anteriormente, a distribuição $t$-Student pode ser utilizada no modelo GARCH quando os dados possuem caudas pesadas. No entanto, mesmo quando se utiliza a distribuição $t$-Student no GARCH, pela especificação da variância condicional ser uma combinação linear de observações ao quadrado, uma consequência é que é muito sensível a observações extremas, e o efeito se dissipa muito lentamente quando estas ocorrem (Harvey, 2013).

Se a equação dinâmica para a volatilidade depender do score condicional da distribuição $t$ Student em um modelo GAS, o modelo resultante diluirá o efeito dos outliers. Tal modelo se transformará no clássico GARCH gaussiano quando os graus de liberdade tendem a infinito.

Sabe-se que o clássico GARCH gaussiano é um caso particular de um modelo GAS, quando os dados têm distribuição normal. Mas se assumirmos que $\epsilon_{t}$ segue uma distribuição $t$-Student com $\nu$ graus de liberdade e variância unitária, a especificação $\operatorname{GAS}(1,1)$ para a variância condicional resulta na equação de evolução a seguir, de acordo com Creal et al. (2013):

$$
\sigma_{t}=\omega+A_{1}\left(1+3 \nu^{-1}\right)\left(\frac{1+\nu^{-1}}{\left(1-2 \nu^{-1}\right)\left(1+\nu^{-1} y_{t-1}^{2} /\left(1-2 \nu^{-1}\right) \sigma_{t-1}\right)} y_{t-1}^{2}-\sigma_{t-1}\right)+B_{1} \sigma_{t-1} .
$$

Se $\nu^{-1}=0$, a distribuição $t$-Student se iguala à distribuição normal e a equação de evolução (5.14) resulta em (5.2). A recursão em (5.14), no entanto, tem uma diferença importante da observada no modelo GARCH(1,1) $t$-Student, que tem exatamente a equação (5.2) para evolução da variância condicional. O denominador do segundo termo do lado direito de (5.14) ocasiona um aumento mais moderado na variância para um valor alto de $\left|y_{t}\right|$, quando $\nu$ é finito. Ou seja, se os erros são modelados por uma distribuição de cauda pesada, uma observação $y_{t}$ com valor absoluto alto não 
necessita de um aumento substancial na variância. Logo, o mecanismo de evolução do GAS para o modelo com erros $t$-Student é substancialmente diferente de seu correspondente GARCH. Em outras palavras, a utilização do score implica em um modelo com uma equação de evolução mais robusta da variância ao longo do tempo.

A Figura 5.2 representa os retornos diários do índice S\&P500, que agrega 500 ativos cotados de 500 grandes empresas dos Estados Unidos, de 30 de outubro de 1985 até 24 de junho de 2016. A Figura 5.3 apresenta a variância condicional em um trecho da série estimada pelos modelos GARCH(1,1) gaussiano, GARCH(1,1) $t$-Student e GAS $(1,1) t$-Student. Os ajustes dos modelos GARCH foram executados pelo pacote fGarch, e do modelo GAS, pelo pacote $G A S$, ambos do software R.

O trecho da série selecionado para exibição da variância condicional fica em torno do ponto 498, que corresponde ao valor extremo negativo que chama a atenção no gráfico da série (valor de $-22,900)$. Como fica claro pelo gráfico, a variância condicional obtida pelo modelo gaussiano é altamente afetada pela observação extrema. O modelo GARCH $t$-Student é menos afetado, e o GAS $t$-Student é ainda mais robusto. Neste caso, o modelo GAS $t$-Student parece ser o mais adequado, pois o processo gerador dos dados parece corresponder a uma distribuição de caudas pesadas. Com a utilização de tal modelo, grandes saltos na variância condicional não são necessários.

$\mathrm{Na}$ série, a variabilidade continua em um patamar elevado pelas sete observações seguintes após a observação 498. Os valores variam entre -8 e 8 , voltando logo depois para o patamar de -3 a 3 . Para os modelos GARCH, tanto gaussiano quanto t-Student, cerca de 100 dias são necessários para que a variância condicional estimada retorne ao patamar que se encontrava antes da observação referida. Ou seja, o efeito do valor extremo leva muito tempo para se dissipar, afetando a variância condicional por um período muito mais longo do que deveria. O modelo GAS não é afetado de forma tão drástica por tal valor extremo, e a variância condicional retorna ao nível anterior muito antes do que os modelos GARCH.

\subsection{Modelo proposto para volatilidade: GAS com distribuição es- tável}

O modelo GARCH estável mencionado na Subseção 5.1.2 não possui o modelo GARCH gaussiano como caso particular, e isso seria uma característica desejável para a proposição de um modelo alternativo.

Além disso, pode-se pensar na função score como mecanismo que guie a dinâmica da volatilidade. Tal mecanismo de evolução do parâmetro da distribuição dos dados que varia no tempo é justificado por Blasques et al. (2015) em termos da divergência de Kullback-Leibler. Os resultados afirmam que, para uma dada escolha de densidade, as dinâmicas do parâmetro variante no tempo guiado pelo score possuem diversas propriedades ótimas. Estruturas de evolução baseadas em modelos guiados por observações, como é o caso do modelo GARCH estável, não necessariamente possuem tais propriedades.

Vale a pena notar que o modelo clássico GARCH gaussiano possui essas propriedades, já que a equação de evolução da volatilidade, medida pela variância condicional, é um caso particular de um mecanismo guiado pelo score.

Ao mesmo tempo, no entanto, nem todas as escolhas para a densidade são similares. Para minimizar a divergência de Kullback-Leibler, é sempre preferível trabalhar com um modelo cuja densidade se aproxime do processo gerador dos dados o mais fielmente possível. Logo, uma boa escolha para a densidade condicional é fundamental.

Se os dados possuem caudas pesadas, pode não ser apropriado trabalhar com um modelo GARCH gaussiano. O modelo é ótimo no aspecto da divergência de Kullback-Leibler, mas a densidade condicional postulada pode estar longe daquela que gera o processo. A distribuição $t$-Student pode ser utilizada, mas os dados podem apresentar comportamento leptocúrtico que pode não ser captado pela distribuição. Além disso, a $t$-Student não possui a propriedade de estabilidade desejável quando as quantidades observadas são somas de pequenas outras quantidades, como pode 


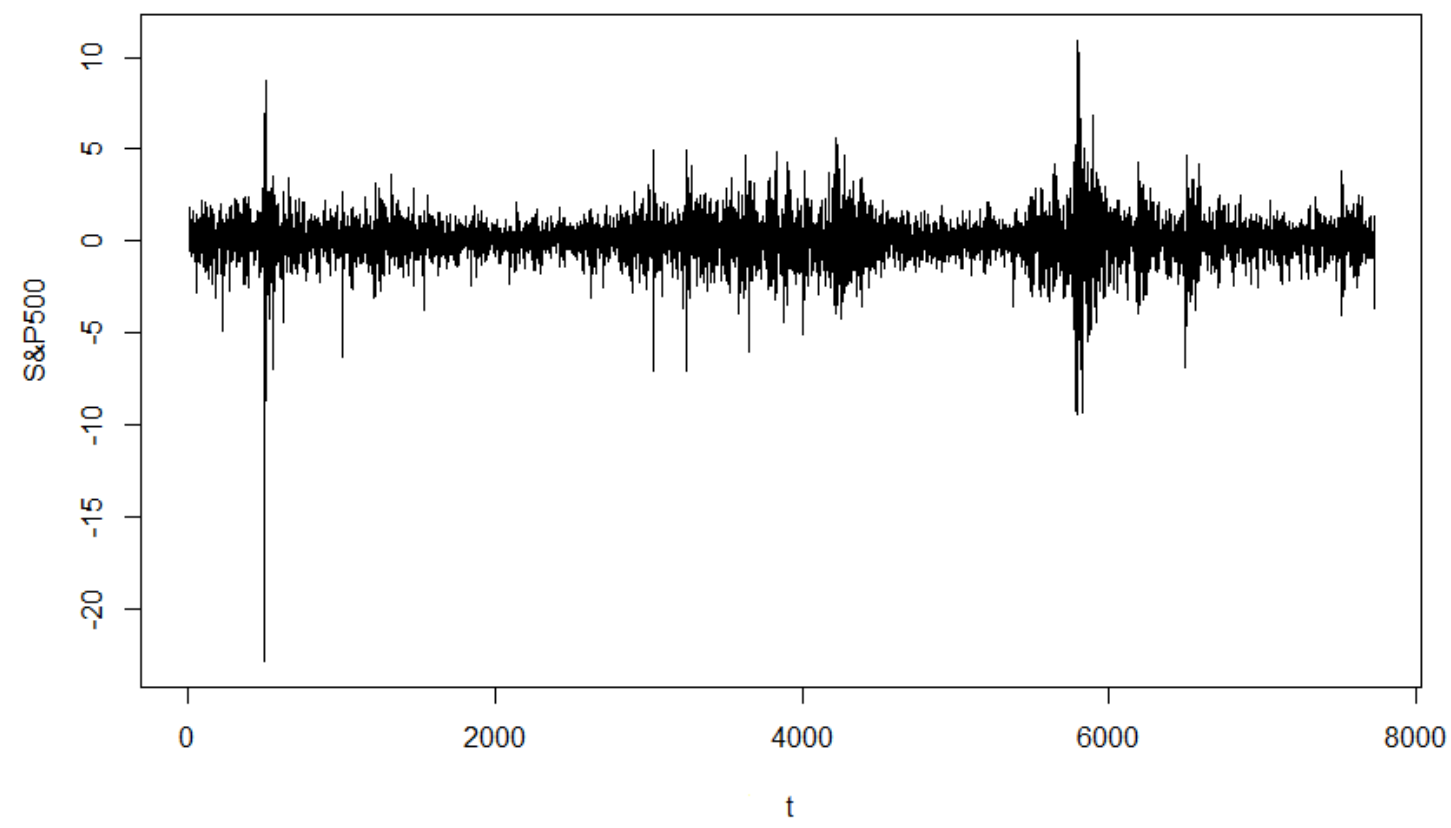

Figura 5.2: Retornos diários de S\&P500 de 30 de outubro de 1985 a 24 de junho de 2016

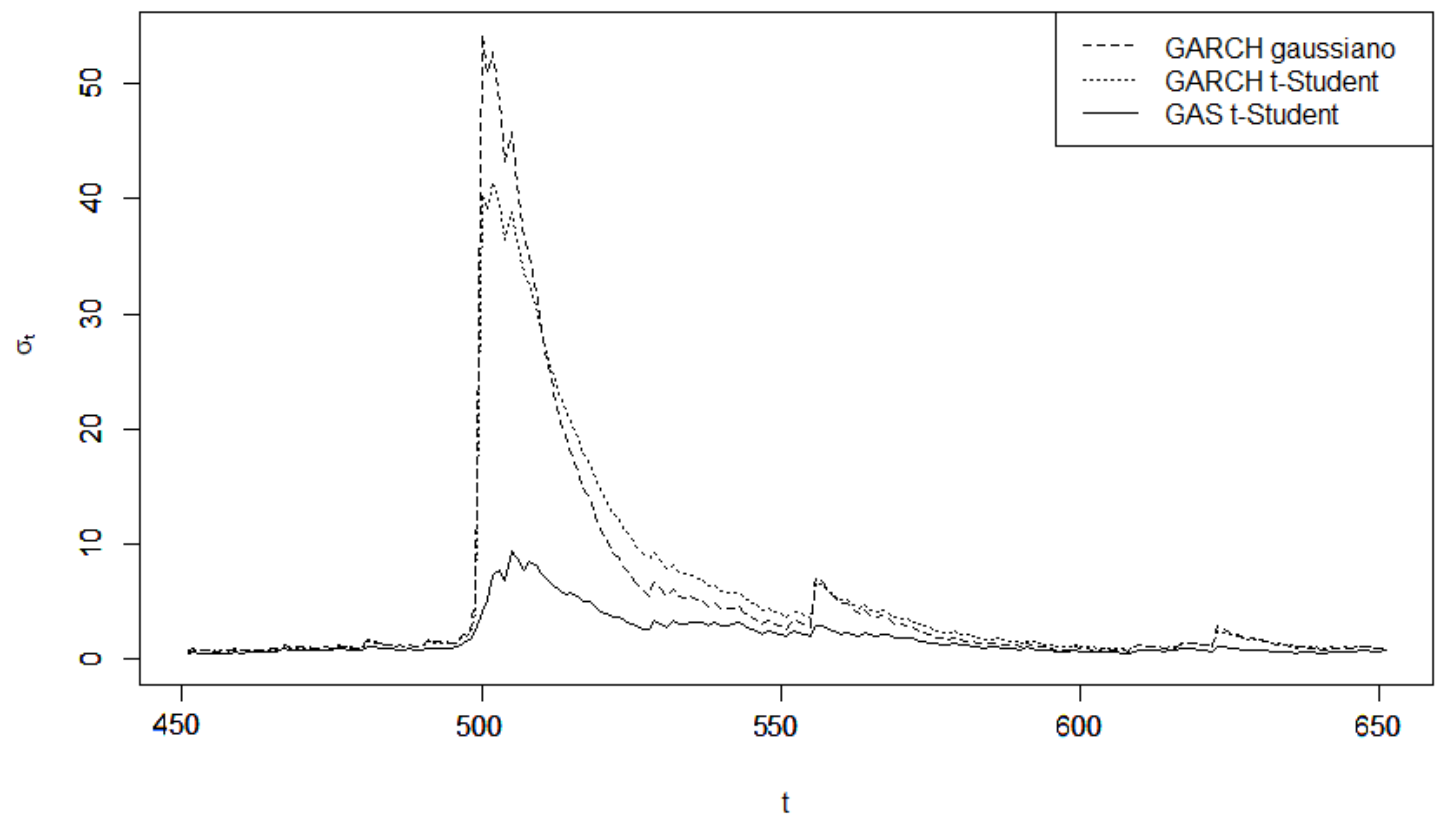

Figura 5.3: Variância condicional dos retornos diários de SछP500 estimada pelos modelos GARCH gaussiano, GARCH t-Student e GAS t-Student em trecho da série 
ocorrer com preço de ativos, ruídos de um sistema de comunicação etc. Outro fator a favor da distribuição estável surge quando há evidências de que os dados não possuem segundo momento finito. Tal comportamento é observado quando são detectados valores à primeira vista aberrantes surgem de maneira muito mais frequente do que o esperado em uma distribuição normal ou até em uma distribuição $t$-Student.

Para adequar as caudas pesadas da distribuição $t$-Student, a estabilidade da distribuição normal, a capacidade de lidar com dados leptocúrticos e as propriedades ótimas oferecidas por um modelo guiado pelo score, é proposto aqui um modelo GAS com distribuição estável. O modelo tem todas as vantagens citadas, além de englobar o modelo clássico GARCH gaussiano como um caso particular. Seu conceito é relativamente simples, mas os cálculos envolvidos, o procedimento de estimação e as condições de estacionariedade podem ser complicados.

\subsubsection{Especificação do modelo}

Seja $y_{t}$ uma série temporal univariada, $t=1, \ldots, T$, com $y_{t}=\sqrt{\sigma_{t}} \epsilon_{t}$, em que o ruído aleatório $\epsilon_{t}$ tem distribuição estável, com parâmetro de localização $\delta=0$, parâmetro de assimetria $\beta=0$ (distribuição simétrica), parâmetro de escala $\gamma=1 / \sqrt{2}$ e parâmetro de estabilidade $\alpha$ (notação: $\left.\epsilon_{t} \sim S_{\alpha}(0,0,1 / \sqrt{2})\right)$. O valor de $1 / \sqrt{2}$ é escolhido para $\gamma$ pelo fato de, quando $\alpha=2$, a distribuição de $\epsilon_{t}$ se tornar a normal padrão. Pelas propriedades das distribuições estáveis, temos que $y_{t} \sim$ $S_{\alpha}(0,0, \sqrt{\sigma / 2})$. Seja $\sigma_{t}$ o parâmetro dinâmico, que se refere à volatilidade dos dados, com equação de evolução de acordo com o modelo $\operatorname{GAS}(p, q)$, com $q \geq p$ :

$$
\sigma_{t}=\omega+\sum_{i=1}^{p} A_{i} s_{t-i}+\sum_{i=1}^{q} B_{j} \sigma_{t-j}
$$

em que

$$
\begin{gathered}
s_{t}=S_{t} \nabla_{t}, \quad \nabla_{t}=\frac{\partial \ln f\left(y_{t} ; \sigma_{t}\right)}{\partial \sigma_{t}}=\frac{\partial \ln f_{t}}{\partial \sigma_{t}}, \\
S_{t}=\mathcal{I}_{t}^{-1}, \quad \mathcal{I}_{t}=\mathbb{E}_{t}\left[\nabla_{t}^{2}\right] .
\end{gathered}
$$

Como o ruído aleatório tem distribuição estável, o modelo é diferente de um GARCH no caso geral. No entanto, para $\alpha=2, \epsilon_{t}$ tem distribuição normal padrão. Conforme discutido na Seção 2.2, nesse caso o modelo se torna um GARCH gaussiano.

Para previsão da volatilidade, o procedimento é o mesmo descrito para o modelo GAS $t$-Student (equação (5.13)). Se $T$ é o tamanho da amostra para a qual o modelo é ajustado, então o valor da previsão $h$ passos à frente de $T$, denotada por $\hat{\sigma}_{T+h}$, é dada por

$$
\hat{\sigma}_{T+h}=\hat{\omega}+\sum_{i=1}^{p} \hat{A}_{i} \hat{s}_{T+h-i}+\sum_{j=1}^{q} \hat{B}_{j} \hat{\sigma}_{T+h-j},
$$

em que $\hat{\omega}, \hat{A}_{i}, i=1, \ldots, p, \hat{B}_{j}, j=1, \ldots, q$ são os valores estimados dos parâmetros do modelo. Se $T+h-k \leq T$, então $\hat{\sigma}_{T+h-k}=\sigma_{T+h-k}$ e $\hat{s}_{T+h-k}=s\left(\hat{y}_{T+h-k}\right)=s\left(y_{T+h-k}\right)$. Caso contrário, $\hat{s}_{T+h-k}$ é obtido pela esperança condicional de $s_{T+h-k}=s\left(y_{T+h-k}\right)$, que, pelas propriedades do score, é zero.

Se $\alpha \neq 2$, os cálculos podem ser complicados, já que a função densidade de probabilidade da distribuição estável correspondente não pode ser escrita em uma forma analítica fechada. Baseado nos trabalhos de DuMouchel (1975), Zolotarev (1986), Nolan (1997) e Matsui e Takemura (2006), é possível efetuar os cálculos para a especificação completa do modelo, bem como para o processo de estimação. Tais cálculos estão descritos no Capítulo 4.

O presente trabalho considera as ordens $p=1$ e $q=1$, já que no caso GARCH ordens baixas são recomendadas. No entanto, os cálculos podem ser estendidos para ordens maiores de maneira análoga. Também se considera $1<\alpha \leq 2$ por duas razões: (i) para $\alpha \leq 1$, a distribuição estável 
não possui momentos, e usualmente dados financeiros, por suposição, possuem ao menos o primeiro momento finito; e (ii) para $\alpha \leq 1$ os cálculos se tornam ainda mais complicados, com características específicas dependendo do intervalo no qual $\alpha$ se encontra.

Conforme descrito na Seção 2.2, a volatilidade é a variabilidade de uma série ao longo do tempo. Como $\sigma_{t}$ é o parâmetro de escala de $y_{t}$, então está associado à variabilidade da série e é uma medida de volatilidade. À exceção do caso $\alpha=2$, não é possível se referir a $\sigma_{t}$ como variância condicional, já que, para $\alpha \neq 2$, a distribuição estável não possui segundo momento finito.

Pelas equações (5.15) e (5.16), pode-se perceber que o valor do parâmetro de escala no instante $t$ depende da derivada da log-verossimilhança com respeito ao parâmetro em instantes anteriores. Tal estrutura de dependência faz o modelo drasticamente diferente do modelo normal, ao menos em um aspecto.

Para efeito de ilustração, a Figura 5.4 exibe o comportamento de funções densidade de duas distribuições estáveis, $\operatorname{com} \alpha=2$ e $\alpha=1,99$ respectivamente. As distribuiçõe são simétricas $(\beta=0)$ em torno de zero $(\delta=0)$. O parâmetro de escala $\gamma=1 / \sqrt{2}$ em ambos os casos, de modo que, para $\alpha=2$, a distribuição se torna a distribuição normal padrão. Percebe-se que, visualmente, é praticamente impossível distinguir uma densidade da outra. Para atestar tal semelhança, os valores de $f(y)$ nos pontos $y=0,1,2$ e 3 quando $\alpha=2$ são, respectivamente, 0,39894, 0,24197, 0,05399 e 0,00443; quando $\alpha=1,99$, os valores são $0,39898,0,24147,0,05393$ e 0,00467 .

Apesar dos comportamentos das densidades serem muito semelhantes, um cenário bem diferente é observado quando se comparam as funções score das duas distribuições em relação a $\gamma$ avaliado no ponto $\gamma=1 / \sqrt{2}$, ou seja, a função $[\partial \ln f(y \mid \gamma) / \partial \gamma]_{\gamma=1 / \sqrt{2}}$. Tal comparação é feita na Figura 5.5.

No caso normal, o score é uma função quadrática de $y$. Por isso, quando o valor de $y$ é muito alto, positivamente ou negativamente, tal derivada terá um valor altíssimo, e com isso é possível notar o impacto que um possível outlier pode ter em (5.16). Por outro lado, no caso estável, a derivada não é uma função quadrática de $y$. Tanto que, à medida que $y$ cresce, a partir de certo momento a derivada decresce e tem seu valor estabilizado. Por isso, um outlier não terá impacto significativo em (5.16). E é notável que tal cenário seja observado para $\alpha=1,99$, o que corresponde a um modelo muito próximo do normal. À primeira vista, a robustez a outliers é uma vantagem do modelo estável, ainda mais destacável quando o valor de $\alpha$ diminui, o que corresponde a caudas mais pesadas.

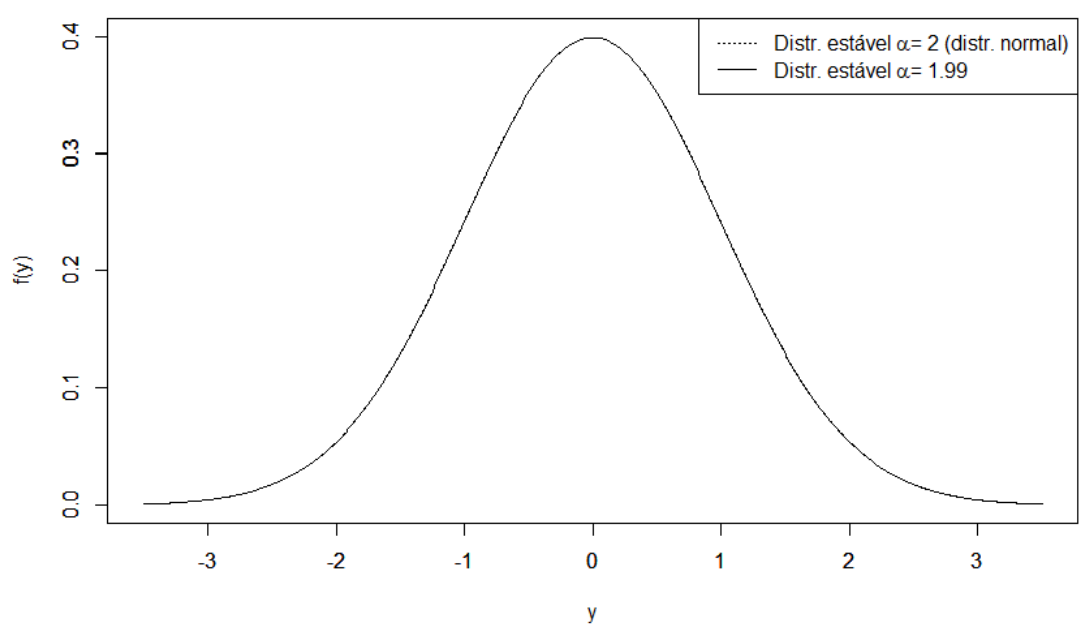

Figura 5.4: Funções densidade de distribuições normal e estável 


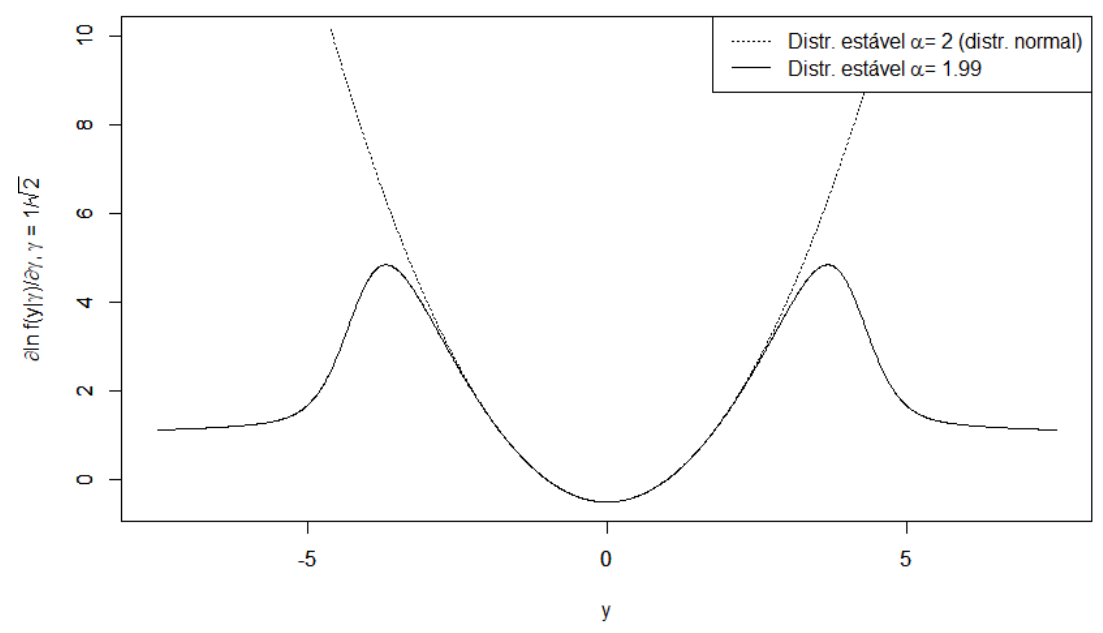

Figura 5.5: Funções score de distribuições normal e estável

\subsubsection{Restrições para os parâmetros}

Um dos aspectos importantes nos modelos GARCH é a imposição de desigualdades para manter a variância condicional não-negativa. Bollerslev (1986) demonstrou que todos os parâmetros estáticos do modelo devem ser iguais ou maiores a zero. Neste caso, é fácil observar que tais condições garantem que a variância condicional permaneça não-negativa. Nelson e Cao (1992) mostraram que desigualdades menos restritivas são suficientes para manter a variância condicional não-negativa.

No modelo GAS com distribuição estável proposto, devemos garantir que $\sigma_{t}$ permaneça nãonegativo. Uma análise da Figura 5.5 mostra que há uma região na qual a função score tem valores negativos. É preciso lidar com isso.

O valor mínimo da função score de uma distribuição estável padrão simétrica é atingido no ponto em que o argumento é igual a zero. O cálculo do valor do score nesse ponto é simples. Pelas equações (4.3) e (4.7), utilizando a mesma notação do Capítulo 4, tem-se

$$
\begin{aligned}
{\left[\frac{\partial \ln f\left(y_{t} ; \gamma\left(\sigma_{t}\right), \alpha\right)}{\partial \sigma_{t}}\right]_{y_{t}=0} } & =\left[l_{\sigma_{t}}\left(y_{t} ; \gamma\left(\sigma_{t}\right), \alpha\right)\right]_{y_{t}=0}=\left[\frac{f_{\sigma_{t}}\left(y_{t} ; \gamma\left(\sigma_{t}\right), \alpha\right)}{f\left(y_{t} ; \gamma\left(\sigma_{t}\right), \alpha\right)}\right]_{y_{t}=0} \\
& =\left[\left(-\frac{\gamma^{\prime}\left(\sigma_{t}\right)}{\gamma\left(\sigma_{t}\right)^{2}} f^{*}\left(\frac{y_{t}}{\gamma\left(\sigma_{t}\right)} ; \alpha\right)-y_{t} \frac{\gamma^{\prime}\left(\sigma_{t}\right)}{\gamma\left(\sigma_{t}\right)^{3}} f^{* \prime}\left(\frac{y_{t}}{\gamma\left(\sigma_{t}\right)} ; \alpha\right)\right) \frac{1}{f\left(y_{t} ; \gamma\left(\sigma_{t}\right), \alpha\right)}\right]_{y_{t}=0} \\
& =\left(-\frac{\gamma^{\prime}\left(\sigma_{t}\right)}{\gamma\left(\sigma_{t}\right)^{2}} f^{*}(0 ; \alpha)\right) \frac{1}{f^{*}\left(0 ; \gamma\left(\sigma_{t}\right), \alpha\right)} \\
& =\left(-\frac{\gamma^{\prime}\left(\sigma_{t}\right)}{\gamma\left(\sigma_{t}\right)^{2}} \gamma\left(\sigma_{t}\right) f\left(0 ; \gamma\left(\sigma_{t}\right), \alpha\right)\right) \frac{1}{f\left(0 ; \gamma\left(\sigma_{t}\right), \alpha\right)}=-\frac{\gamma^{\prime}\left(\sigma_{t}\right)}{\gamma\left(\sigma_{t}\right)}
\end{aligned}
$$

Como $\gamma\left(\sigma_{t}\right)=\sqrt{\sigma_{t} / 2}$, então $\gamma^{\prime}\left(\sigma_{t}\right)=1 /\left(2 \sqrt{2 \sigma_{t}}\right)$. Logo,

$$
\left[\frac{\partial \ln f\left(y_{t} ; \gamma\left(\sigma_{t}\right), \alpha\right)}{\partial \sigma_{t}}\right]_{y_{t}=0}=-\frac{1}{2 \sigma_{t}} .
$$

Pela equação (4.64),

$$
\mathcal{I}_{t}=\frac{\mathcal{I}_{t}^{s t d}}{4 \sigma_{t}^{2}}
$$

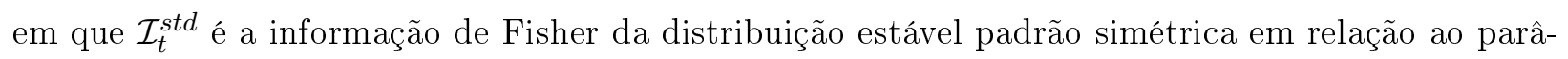
metro de escala. 
Logo, $\sigma_{t}$ tem seu valor mínimo em

$$
\sigma_{t}=\omega+\sum_{i=1}^{p} A_{i}\left(-\frac{2 \sigma_{t-i}}{\mathcal{I}_{t}^{s t d}}\right)+\sum_{j=1}^{q} B_{j} \sigma_{t-j}=\omega+\sum_{i=1}^{q}\left(-\frac{2 A_{i}}{\mathcal{I}_{t}^{s t d}}+B_{i}\right) \sigma_{t-i},
$$

considerando $\omega \geq 0, A_{i} \geq 0$ e $B_{i} \geq 0$. Também, $A_{i}=0$ para $i>p$.

Logo, uma condição suficiente para $\sigma_{t} \geq 0$ é

$$
\omega, A_{i} \geq 0 \text { e } B_{i} \geq \frac{2 A_{i}}{\mathcal{I}_{t}^{s t d}}, \quad i=1, \ldots, q .
$$

Exemplo 5.2.1 Se $\alpha=2$, o modelo se torna um modelo $G A R C H(p, q)$ gaussiano. Como nesse caso $\mathcal{I}_{t}^{\text {std }}=2$, a condição se torna $\omega \geq 0, A_{i} \geq 0, B_{i} \geq 0$ e $B_{i}-A_{i} \geq 0$. O resultado está de acordo com a conhecida condição para o modelo GARCH gaussiano, descrita na Subseção 5.1.1.

\subsubsection{Condições de estacionariedade}

Na Seção 2.4, foi mostrado como Blasques et al. (2014a) determinaram condições para estacionariedade de modelos GAS. No entanto, para se obter condições de estacionariedade de modelos GAS que utilizam distribuições estáveis, tal procedimento não é o mais adequado. Simplesmente porque não há fórmulas explícitas para as densidades de tais distribuições, e não é possível calcular analiticamente as expressões necessárias para a obtenção das condições de estacionariedade mencionadas.

No modelo GAS clássico em (2.3)-(2.5), como $\nabla_{t}$ é o score da função densidade de probabilidade com relação a $\sigma_{t}$, segue que $\mathbb{E}_{t}\left[s_{t}\right]=0$ e $s_{t}$ forma uma sequência de diferença martingal. O processo $\sigma_{t}$ pode então ser escrito como um processo média móvel infinito de diferenças martingais. No caso do $\operatorname{GAS}(1,1)$, tem-se

$$
\sigma_{t}=\omega+\sum_{i=1}^{\infty} B_{1}^{i-1} A_{1} s_{t-i}
$$

Se a variância de $s_{t}$ é constante ao longo do tempo, o processo $\sigma_{t}$ é estacionário de segunda ordem quando a raiz do polinômio $1-B_{1}$ estiver dentro do círculo unitário, ou seja, quando $\left|B_{1}\right|<1$ (no caso do processo ter segundo momento finito, o que em geral não ocorre com distribuições estáveis). Em geral, no entanto, quando se usa o inverso da informação como fator de ponderação, ou seja, $S_{t}=\mathcal{I}_{t}^{-1}$, a variância de $s_{t}$ não é constante, e formular condições de estacionariedade é mais difícil (Creal et al., 2011).

Como o objetivo aqui é ter uma estrutura semelhante ao do modelo GARCH, o que requer que o score seja ponderado por $\mathcal{I}_{t}^{-1}$, há dois aspectos importantes: lidar com $\mathcal{I}_{t}^{-1}$ e tratar a estacionariedade de forma estrita. Como o modelo proposto considera uma distribuição estável como distribuição condicional, não faz sentido falar em estacionariedade de segunda ordem, por causa da ausência de segundo momento.

Pode-se associar com o processo GAS uma equação multivariada de diferença estocástica do seguinte tipo:

$$
\boldsymbol{X}_{t}=\boldsymbol{W}_{t-1} \boldsymbol{X}_{t-1}+\boldsymbol{V}_{t-1}, \quad t \in \mathbb{Z},
$$

em que $\boldsymbol{X}_{t}$ e $\boldsymbol{V}_{t}$ são vetores aleatórios em $\mathbb{Z}^{d}, \boldsymbol{W}_{t}$ é matriz $d \times d$ e $\left\{\left(\boldsymbol{W}_{t}, \boldsymbol{V}_{t}\right), t \in \mathbb{Z}\right\}$ é um processo estritamente estacionário ergódico. Dessa forma, as propriedades de estacionariedade do processo GAS estão diretamente relacionadas às propriedades de estacionariedade das soluções de (5.24). É, então, suficiente considerar esse modelo geral para tais propósitos.

Brandt (1986) obteve condições suficientes para estacionariedade estrita do processo representado por (5.24). Bougerol e Picard (1992) e Mittnik et al. (2002) escreveram os processos GARCH clássicos e stable-power-GARCH, respectivamente, na representação (5.24) e obtiveram condições necessárias e suficientes para estacionariedade. Para isso, se utilizaram da não-negatividade da matriz $W_{t}$. Para escrever o modelo GAS com distribuição estável na representação (5.24), como poderá 
ser verificdo a seguir, a matriz $W_{t}$ não é necessariamente não-negativa. Por isso, serão obtidas a seguir condições apenas suficientes.

Pela equação (4.64), tem-se

$$
\mathcal{I}_{t}=\frac{\mathcal{I}_{t}^{s t d}}{4 \sigma_{t}^{2}}
$$

em que $\mathcal{I}_{t}^{s t d}$ é a informação no caso padrão e simétrico. Então a equação de evolução de $\sigma_{t}$, definida por

$$
\sigma_{t}=\omega+\sum_{i=1}^{p} A_{i} s_{t-i}+\sum_{j=1}^{q} B_{j} \sigma_{t-j}=\omega+\sum_{i=1}^{p} A_{i} \mathcal{I}_{t-i}^{-1} \frac{\partial \ln f\left(y_{t-i} ; \sigma_{t-i}\right)}{\partial \sigma_{t-i}}+\sum_{j=1}^{q} B_{j} \sigma_{t-j}
$$

pode ser escrita como

$$
\sigma_{t}=\omega+\sum_{i=1}^{p} A_{i} \frac{4 \sigma_{t-i}^{2}}{\mathcal{I}_{t-i}^{s t d}} \frac{\partial \ln f\left(y_{t-i} ; \sigma_{t-i}\right)}{\partial \sigma_{t-i}}+\sum_{j=1}^{q} B_{j} \sigma_{t-j} .
$$

Note que

$$
\frac{\partial \ln f\left(y_{t} ; \sigma_{t}\right)}{\partial \sigma_{t}}=\frac{1}{f\left(y_{t} ; \sigma_{t}\right)} \frac{\partial f\left(y_{t} ; \sigma_{t}\right)}{\partial \sigma_{t}} .
$$

Como $y_{t} \sim S_{\alpha}\left(0,0, \sqrt{\sigma_{t} / 2}\right)$, então

$$
f\left(y_{t} ; \sigma_{t}\right)=\sqrt{\frac{2}{\sigma_{t}}} f^{*}\left(\sqrt{\frac{2}{\sigma_{t}}} y_{t}\right)
$$

em que $f^{*}$ é a densidade da distribuição estável padrão no caso simétrico. Logo,

$$
\frac{\partial f\left(y_{t} ; \sigma_{t}\right)}{\partial \sigma_{t}}=-\frac{\sigma_{t}^{-3 / 2}}{\sqrt{2}} f^{*}\left(\sqrt{\frac{2}{\sigma_{t}}} y_{t}\right)-y_{t} \sigma_{t}^{-2} f^{* \prime}\left(\sqrt{\frac{2}{\sigma_{t}}} y_{t}\right)
$$

e

$$
\frac{\partial \ln f\left(y_{t}\right)}{\partial \sigma_{t}}=-\frac{\sigma_{t}^{-1}}{2}-\frac{y_{t}}{\sqrt{2}} \sigma^{-3 / 2} \frac{f^{* \prime}\left(\sqrt{\frac{2}{\sigma_{t}}} y_{t}\right)}{f^{*}\left(\sqrt{\frac{2}{\sigma_{t}}} y_{t}\right)} .
$$

Como $y_{t}=\sqrt{\sigma_{t}} \epsilon_{t}$, então

$$
\frac{\partial \ln f\left(y_{t} ; \sigma_{t}\right)}{\partial \sigma_{t}}=\sigma_{t}^{-1}\left(-\frac{1}{2}-\frac{\epsilon_{t}}{\sqrt{2}} \frac{f^{* \prime}\left(\sqrt{2} \epsilon_{t}\right)}{f^{*}\left(\sqrt{2} \epsilon_{t}\right)}\right),
$$

e, substituindo em (5.26),

$$
\sigma_{t}=\omega+\sum_{i=1}^{p} A_{i} \frac{4 \sigma_{t-i}}{\mathcal{I}_{t-i}^{s t d}}\left(-\frac{1}{2}-\frac{\epsilon_{t-i}}{\sqrt{2}} \frac{f^{* \prime}\left(\sqrt{2} \epsilon_{t-i}\right)}{f^{*}\left(\sqrt{2} \epsilon_{t-i}\right)}\right)+\sum_{j=1}^{q} B_{j} \sigma_{t-j} .
$$

Como $q \geq p$, o modelo proposto pode ser escrito como

$$
\boldsymbol{X}_{t}=\boldsymbol{W}_{t-1} \boldsymbol{X}_{t-1}+\boldsymbol{V}_{t-1}, \quad t \in \mathbb{Z}
$$

em que a $k$-ésima componente do vetor $\boldsymbol{X}_{t}$, denotada por $X_{k, t}$, é dada por

$$
X_{k, t}= \begin{cases}\sigma_{t}, & \text { se } k=1, \\ \sum_{i=k}^{q} A_{i} s_{t+k-1-i}+\sum_{j=k}^{q} B_{j} \sigma_{t+k-1-j}, & \text { se } k=2, \ldots, q,\end{cases}
$$


$\operatorname{com} A_{i}=0$ se $i>p$.

$\boldsymbol{W}_{t}$ e $\boldsymbol{V}_{t}$ são definidos por

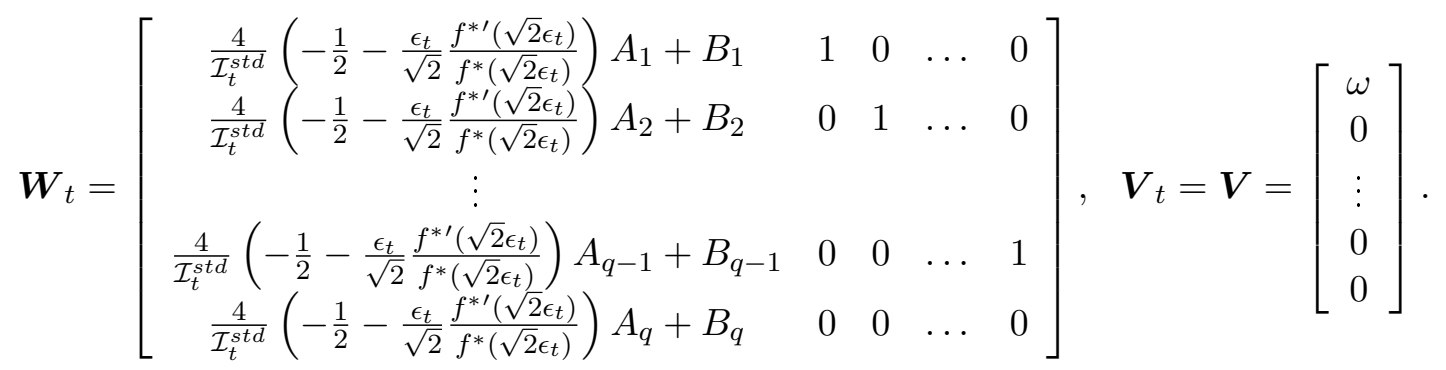

Claramente $\left\{\left(\boldsymbol{W}_{t}, \boldsymbol{V}_{t}\right), t \in \mathbb{Z}\right\}$ é um processo estritamente estacionário. No entanto, conforme mencionado, $\boldsymbol{W}_{t}$ não necessariamente é não-negativa, fazendo com que não seja possível utilizar as estratégias adotadas por Bougerol e Picard (1992) e Mittnik et al. (2002) para que se obtenham condições suficientes e necessárias. Vamos nos ater às condições suficientes.

Definição 5.2.1 Seja $\|$.$\| uma norma em \mathbb{R}^{d}$, e seja um operador de norma no conjunto $\mathbb{M}(d)$ de matrizes $d \times d$ por

$$
\|\boldsymbol{M}\|=\sup \left\{\|\boldsymbol{M} \boldsymbol{x}\| /\|\boldsymbol{x}\| ; \boldsymbol{x} \in \mathbb{R}^{d}, \boldsymbol{x} \neq \mathbf{0}\right\}
$$

para qualquer $\boldsymbol{M}$ em $\mathbb{M}(d)$. Então o Expoente Máximo de Lyapunov (EML) associado à sequência $\left\{\boldsymbol{W}_{t}, t \in \mathbb{Z}\right\}$ de matrizes aleatórias i.i.d. é definido por

$$
\tau=\inf \left\{\mathbb{E}\left[\frac{1}{n+1} \ln \left\|\boldsymbol{W}_{0} \boldsymbol{W}_{-1} \ldots \boldsymbol{W}_{-t}\right\|\right], t \in \mathbb{N}\right\}
$$

em que $\mathbb{E}\left[\ln ^{+}\left\|\boldsymbol{W}_{0}\right\|\right]$ é finito $\left(\ln ^{+} x=\max (\ln x, 0)\right)$.

Teorema 5.2.1 Se $\left\{\boldsymbol{W}_{t}\right\}$ é uma sequência estacionária com $\mathbb{E}\left[\ln ^{+}\left\|\boldsymbol{W}_{0}\right\|\right]<\infty$, então

$$
\tau=\lim _{n \rightarrow+\infty} \frac{1}{n} \ln \left\|\boldsymbol{W}_{0} \boldsymbol{W}_{-1} \ldots \boldsymbol{W}_{-t}\right\| .
$$

A demonstração do Teorema 5.2.1 foge do escopo deste texto e pode ser encontrada em Kingman (1973). O valor de $\tau$ é independente da norma utilizada (Bougerol e Picard, 1992).

Teorema 5.2.2 Seja $\left\{\left(\boldsymbol{W}_{t}, \boldsymbol{V}_{t}\right), t \in \mathbb{Z}\right\}$ um processo estritamente estacionário ergódico tal que ambos $\mathbb{E}\left[\ln ^{+}\left\|\boldsymbol{W}_{0}\right\|\right]$ e $\mathbb{E}\left[\ln ^{+}\left\|\boldsymbol{V}_{0}\right\|\right]$ são finitos. Suponha que o EML $\tau$ é estritamente negativo. Então, para todo $t \in \mathbb{Z}$, a sequência

$$
\boldsymbol{X}_{t}=\sum_{i=0}^{\infty} \boldsymbol{W}_{t} \boldsymbol{W}_{t-1} \ldots \boldsymbol{W}_{t-i+1} \boldsymbol{V}_{t-i}
$$

converge quase certamente e o processo $\left\{\boldsymbol{X}_{t}, t \in \mathbb{Z}\right\}$ é a única solução estritamente estacionária de (5.24).

Prova: Seja $t$ um inteiro. Como $\boldsymbol{V}_{t}$ é constante para todo $t\left(\boldsymbol{V}_{t}=\boldsymbol{V}=\boldsymbol{V}_{0}\right)$, então $\mathbb{E}\left[\ln ^{+}\left\|\boldsymbol{V}_{0}\right\|\right]<$ $\infty$. Logo,

$$
\sum_{k=0}^{\infty} \mathbb{P}\left(\ln ^{+}\|\boldsymbol{V}\|>-\frac{k \tau}{2}\right)<\mathbb{E}\left[\ln ^{+}\|\boldsymbol{V}\|\right]<\infty
$$


Isso implica, pelo lema de Borel-Cantelli, que

$$
\begin{aligned}
& \mathbb{P}\left(\limsup \ln ^{+}\|\boldsymbol{V}\|>-\frac{k \tau}{2}\right)=0 \\
\Rightarrow & \mathbb{P}\left(\limsup \frac{1}{k} \ln ^{+}\|\boldsymbol{V}\|>-\frac{\tau}{2}\right)=0 \\
\Rightarrow & \limsup \frac{1}{k} \ln ^{+}\|\boldsymbol{V}\| \leq-\frac{\tau}{2}
\end{aligned}
$$

Somando (5.39) e (5.42) tem-se

$$
\limsup \frac{1}{k} \ln \left\|\boldsymbol{W}_{t} \boldsymbol{W}_{t-1} \ldots \boldsymbol{W}_{t-k+1}|| \mid \boldsymbol{V}\right\| \leq \tau / 2<0 .
$$

Pela desigualdade de Cauchy-Schwarz,

$$
\begin{aligned}
& \limsup \frac{1}{k} \ln \left\|\boldsymbol{W}_{t} \boldsymbol{W}_{t-1} \ldots \boldsymbol{W}_{t-k+1} \boldsymbol{V}\right\| \leq 0 \\
\Rightarrow & \limsup \ln \left\|\boldsymbol{W}_{t} \boldsymbol{W}_{t-1} \ldots \boldsymbol{W}_{t-k+1} \boldsymbol{V}\right\|^{1 / k} \leq 0 \\
\Rightarrow & \limsup \left\|\boldsymbol{W}_{t} \boldsymbol{W}_{t-1} \ldots \boldsymbol{W}_{t-k+1} \boldsymbol{V}\right\|^{1 / k} \leq 1 .
\end{aligned}
$$

Pelo critério da raiz de Cauchy, $\sum_{k=1}^{\infty} \boldsymbol{W}_{t} \boldsymbol{W}_{t-1} \ldots \boldsymbol{W}_{t-k+1} \boldsymbol{V}$ converge quase certamente. Essa sequência é uma solução de (5.24) e é estritamente estacionária.

Como $\sum_{k=1}^{\infty} \boldsymbol{W}_{t} \boldsymbol{W}_{t-1} \ldots \boldsymbol{W}_{t-k+1} V$ converge quase certamente, então $\boldsymbol{W}_{t} \boldsymbol{W}_{t-1} \ldots \boldsymbol{W}_{t-k+1} \boldsymbol{V}$ converge para zero quase certamente. Pode ser mostrado que

$$
\lim _{t \rightarrow \infty} \boldsymbol{W}_{t} \boldsymbol{W}_{t-1} \ldots \boldsymbol{W}_{t-k+1}=0
$$

quase certamente (Bougerol e Picard, 1992). Seja $\left\{\boldsymbol{Z}_{t}\right\}$ outra solução estritamente estacionária de (5.24). A relação, para $t \geq 0$,

$$
\left\|\boldsymbol{X}_{0}-\boldsymbol{Z}_{0}\right\|=\left\|\boldsymbol{W}_{0} \ldots \boldsymbol{W}_{-t}\left(\boldsymbol{X}_{-t-1}-\boldsymbol{Z}_{-t-1}\right)\right\| \leq\left\|\boldsymbol{W}_{0} \ldots \boldsymbol{W}_{-t}\right\|\left\|\boldsymbol{X}_{-t-1}-\boldsymbol{Z}_{-t-1}\right\|
$$

o fato que $\left\|\boldsymbol{W}_{0} \ldots \boldsymbol{W}_{-t}\right\|$ converge para 0 , quase certamente, e o fato que a lei de $\boldsymbol{X}_{-t-1}-\boldsymbol{Z}_{-t-1}$ é independente de $t$ implicam que $\boldsymbol{X}_{0}-\boldsymbol{Z}_{0}$ converge para $\mathbf{0}$ em probabilidade. Logo, $\boldsymbol{X}_{0}=\boldsymbol{Z}_{0}$. Isso mostra que a solução é única, o que completa a prova.

Proposição 5.2.1 O processo GAS estável tem uma solução estritamente estacionária única se

$$
\sum_{j=1}^{q} B_{j}<1
$$

Prova: Sejam $m \in \mathbb{N}^{+}, a_{i} \in \mathbb{R}, i=1, \ldots, m$ e a matriz $\boldsymbol{A}$ dada por

$$
\boldsymbol{A}=\left[\begin{array}{ccccc}
a_{1} & 1 & 0 & \ldots & 0 \\
a_{2} & 0 & 1 & \ldots & 0 \\
\vdots & & & & \\
a_{m-1} & 0 & 0 & \ldots & 1 \\
a_{m} & 0 & 0 & \ldots & 0
\end{array}\right]
$$

O determinante de $z \boldsymbol{I}_{m}-\boldsymbol{A}$, em que $\boldsymbol{I}_{m}$ é a matriz identidade de dimensão $m \times m$, é dado por

$$
\operatorname{Det}\left(z I_{m}-\boldsymbol{A}\right)=z^{m}\left(1-\sum_{i=1}^{m} a_{i} z^{-i}\right) .
$$


Logo,

$$
\operatorname{Det}\left(z \boldsymbol{I}_{q}-\mathbb{E}\left[\boldsymbol{W}_{t}\right]\right)=z^{q}\left(1-\mathbb{E}\left[\frac{4}{\mathcal{I}_{t}^{s t d}}\left(-\frac{1}{2}-\frac{\epsilon_{t}}{\sqrt{2}} \frac{f^{* \prime}\left(\sqrt{2} \epsilon_{t}\right)}{f^{*}\left(\sqrt{2} \epsilon_{t}\right)}\right)\right] \sum_{i=1}^{q} A_{i} z^{-i}-\sum_{j=1}^{q} B_{j} z^{-j}\right) .
$$

Segue da desigualdade $|a-b| \geq|(|a|-|b|)|$ que, se $|z|>1$, então

$$
\left|\operatorname{Det}\left(z \boldsymbol{I}_{q}-\mathbb{E}\left[\boldsymbol{W}_{t}\right]\right)\right|>1-\mathbb{E}\left[\frac{4}{\mathcal{I}_{t}^{\text {std }}}\left(-\frac{1}{2}-\frac{\epsilon_{t}}{\sqrt{2}} \frac{f^{* \prime}\left(\sqrt{2} \epsilon_{t}\right)}{f^{*}\left(\sqrt{2} \epsilon_{t}\right)}\right)\right] \sum_{i=1}^{q} A_{i}-\sum_{j=1}^{q} B_{j} .
$$

A seguinte igualdade é válida:

$$
\int \epsilon_{t} f^{* \prime}\left(\sqrt{2} \epsilon_{t}\right) d \epsilon_{t}=-\frac{1}{2}
$$

Tal igualdade é difícil de se verificar analiticamente. O valor foi obtido após diversas checagens numéricas, com variados valores de $\alpha$. Com isso,

$$
\mathbb{E}\left[\epsilon_{t} \frac{f^{* \prime}\left(\sqrt{2} \epsilon_{t}\right)}{f^{*}\left(\sqrt{2} \epsilon_{t}\right)}\right]=\sqrt{2} \int \epsilon_{t} f^{* \prime}\left(\sqrt{2} \epsilon_{t}\right) d \epsilon_{t}=-\frac{\sqrt{2}}{2} .
$$

Logo,

$$
\mathbb{E}\left[\frac{4}{\mathcal{I}_{t}^{s t d}}\left(-\frac{1}{2}-\frac{\epsilon_{t}}{\sqrt{2}} \frac{f^{* \prime}\left(\sqrt{2} \epsilon_{t}\right)}{f^{*}\left(\sqrt{2} \epsilon_{t}\right)}\right)\right]=0
$$

e

$$
\left|\operatorname{Det}\left(z \boldsymbol{I}_{q}-\mathbb{E}\left[\boldsymbol{W}_{t}\right]\right)\right|>1-\sum_{j=1}^{q} B_{j}
$$

Se $\sum_{j=1}^{q} B_{j}<1$, então o raio espectral $\rho$ (o maior autovalor em valor absoluto) de $\mathbb{E}\left[W_{t}\right]$ satisfaz $\rho<1$. O EML $\tau$ sempre satisfaz $\tau \leq \ln \rho$. A prova dessa afirmação pode ser encontrada em Kesten e Spitzer (1984) e está fora do escopo deste trabalho. Logo, $\tau<0$, o que completa a prova.

Exemplo 5.2.2 Quando $\alpha=2$, a distribuição estável torna-se a normal, e o modelo GAS torna-se o clássico modelo GARCH gaussiano quando $q \geq p$. Nesse caso, a equação da variância condicional pode ser escrita como

$$
\sigma_{t}=\omega+\sum_{i=1}^{p} A_{i} y_{t-i}^{2}+\sum_{j=1}^{q}\left(B_{j}-A_{j}\right) \sigma_{t-j} .
$$

De acordo com as condiçôes conhecidas de estacionariedade do modelo GARCH (Morettin, 2011), deve-se ter $\sum_{i=1}^{p} A_{i}+\sum_{j=1}^{q}\left(B_{j}-A_{j}\right)=\sum_{j=1}^{q} B_{j}<1$, exatamente a mesma condição obtida por $(5.47)$.

\subsubsection{Estimação}

O estimador de máxima verossimilhança, tanto para modelos GAS quanto para distribuições estáveis, é consistente e tem distribuição assintótica normal, conforme mencionado nos Capítulos 2 e 3 . O processo de estimação por máxima verossimilhança dos parâmetros $\omega, A_{1}, B_{1}$ e $\alpha$, aqui descrito para o modelo GAS $(1,1)$ estável, é baseado no algoritmo de Newton-Raphson. 
Seja $\boldsymbol{\xi}=\left(\omega, A_{1}, B_{1}, \alpha\right)^{\prime}$. O valor da estimativa de $\boldsymbol{\xi}$ na $k$-ésima iteração do método de NewtonRaphson é representada por

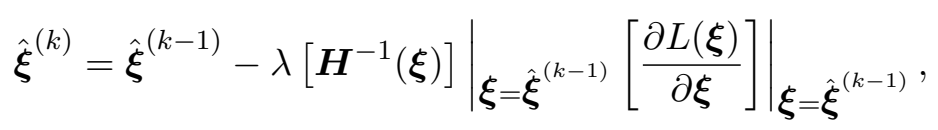

em que $\hat{\boldsymbol{\xi}}^{(k)}$ é o valor estimado do parâmetro após $k$ iterações, $L$ é a função de log-verossimilhança do modelo (sua primeira derivada em relação a $\boldsymbol{\xi}$ é o vetor gradiente) dada por

$$
L(\boldsymbol{\xi})=\sum_{t=1}^{n} l_{t}(\boldsymbol{\xi})=\sum_{t=1}^{n} \ln f_{t}(\boldsymbol{\xi}),
$$

$\boldsymbol{H}$ é a matriz Hessiana formada pelas segundas derivadas da função de log-verossimilhança em relação a $\boldsymbol{\xi}$ dada por

$$
\boldsymbol{H}(\boldsymbol{\xi})=\sum_{t=1}^{n} \frac{\partial^{2} l_{t}(\boldsymbol{\xi})}{\partial \boldsymbol{\xi} \partial \boldsymbol{\xi}^{\prime}}
$$

e $\lambda$ é um número real escolhido de forma apropriada que determina o tamanho do passo do algoritmo na direção estabelecida pelo vetor gradiente e pela matriz Hessiana. A utilização de tal parâmetro define o que se denomina de método de Newton-Raphson relaxado (Gilbert, 2001). Se $\lambda=1$, o algoritmo se reduz ao clássico método de Newton-Raphson.

De acordo com Fiorentini et al. (1996), é possível determinar $\lambda$ maximizando a função de logverossimilhança em uma procura univariada na direção selecionada. Neste trabalho, consideram-se valores de $\lambda$ em um grid no intervalo $[0,1]$, em cada iteração. O valor ótimo de $\lambda$ escolhido na $k$-ésima iteração, digamos $\lambda^{(k)}$, é aquele que, entre os valores considerados no grid, maximiza a log-verossimilhança.

O algoritmo é muito sensível ao valor de iniciaçização de $\hat{\boldsymbol{\xi}}$, e tipicamente não converge se o valor escolhido não está suficientemente próximo ao valor real do estimador de máxima verossimilhança. A escolha de $\lambda$ pelo procedimento descrito parece acelerar os cálculos e melhorar a convergência na maioria dos casos. Neste trabalho, valores de $\hat{\boldsymbol{\xi}}$ calculados para o modelo GARCH $t$-Student (pelo pacote $f$ Garch do $\mathrm{R}$ ) são considerados para a inicialização. O modelo $t$-Student é utilizado ao invés do modelo gaussiano, pois o primeiro é menos sensível a valores extremos, que podem estar presentes nos conjuntos de dados de interesse, que possuem caudas pesadas.

A seguir, os cálculos do gradiente e da Hessiana são detalhados.

Seja

$$
L(\boldsymbol{\xi})=\sum_{t=1}^{T} l_{t}(\boldsymbol{\xi}),
$$

em que $\boldsymbol{\xi}=\left(\omega, A_{1}, B_{1}, \alpha\right)^{\prime}$ e $l_{t}=\ln f\left(y_{t}, \gamma\left(\sigma_{t}\right)\right)$. O vetor gradiente, formado pela derivada de $L$, é a soma das derivadas de $l_{t}$ :

$$
\frac{\partial L(\boldsymbol{\xi})}{\partial \boldsymbol{\xi}}=\sum_{t=1}^{T} \frac{\partial l_{t}}{\partial \boldsymbol{\xi}} .
$$

Seja $\boldsymbol{\xi}=\left(\boldsymbol{\xi}_{1}, \alpha\right)$ em que $\boldsymbol{\xi}_{1}=\left(\omega, A_{1}, B_{1}\right)^{\prime}$ e, pela regra da cadeia,

$$
\frac{\partial l_{t}}{\partial \boldsymbol{\xi}_{1}}=\frac{\partial l_{t}}{\partial \sigma_{t}} \frac{\partial \sigma_{t}}{\partial \boldsymbol{\xi}_{1}}
$$

$\partial l_{t} / \partial \sigma_{t}$ pode ser calculado como explicado no Capítulo 4. Agora é preciso calcular $\partial \sigma_{t} / \partial \boldsymbol{\xi}_{1}$.

Como, pela equação (5.15),

$$
\sigma_{t}=\omega+A_{1} \frac{\partial \ln f_{t}}{\partial \sigma_{t-1}} \mathcal{I}_{t}^{-1}+B_{1} \sigma_{t-1}=\boldsymbol{\xi}^{\prime} \boldsymbol{z}_{t}
$$


em que

$$
\boldsymbol{z}_{t}=\left(1, \frac{\partial \ln f_{t}}{\partial \sigma_{t-1}} \mathcal{I}_{t}^{-1}, \sigma_{t-1}\right)^{\prime}
$$

então

$$
\frac{\partial \sigma_{t}}{\partial \boldsymbol{\xi}_{1}}=\boldsymbol{z}_{t}+B_{1} \frac{\partial \sigma_{t-1}}{\partial \boldsymbol{\xi}_{1}}+A_{1} \frac{\partial \sigma_{t-1}}{\partial \boldsymbol{\xi}_{1}}\left(\frac{\partial^{2} l_{t-1}}{\partial \sigma_{t-1}^{2}} \mathcal{I}_{t}^{-1}-\frac{\partial l_{t-1}}{\partial \sigma_{t-1}} \frac{1}{\mathcal{I}_{t}^{2}} \frac{\partial \mathcal{I}_{t}}{\partial \sigma_{t-1}}\right),
$$

em que $\partial \sigma_{t} / \partial \boldsymbol{\xi}_{1}=0$ for $t \leq 0$, analogamente ao procedimento de estimação de um modelo GARCH (Fiorentini et al., 1996). $\partial^{2} l_{t} / \partial \sigma_{t}^{2}$ pode ser calculado conforme explicado no Capítulo 4.

Para completar $\boldsymbol{\xi}$, é preciso calcular $\partial l_{t} / \partial \alpha$, que, pela regra da cadeia, é igual a

$$
\frac{\partial l_{t}}{\partial \alpha}=\frac{1}{f_{t}} \frac{\partial f_{t}}{\partial \alpha}=\frac{1}{f^{*}\left(y_{t} / \gamma\left(\sigma_{t}\right)\right)} \frac{\partial f^{*}\left(y_{t} / \gamma\left(\sigma_{t}\right)\right)}{\partial \alpha},
$$

em que $f^{*}$ é a densidade de uma distribuição estável padrão simétrica com parâmetro $\alpha$.

A matriz Hessiana é dada por

$$
\boldsymbol{H}(\boldsymbol{\xi})=\sum_{t=1}^{T}\left[\begin{array}{cc}
\frac{\partial^{2} l_{t}}{\partial \boldsymbol{\xi}_{1} \partial \boldsymbol{\xi}_{1}^{\prime}} & \frac{\partial^{2} l_{t}}{\partial \boldsymbol{\xi}_{1} \partial \alpha} \\
\frac{\partial^{2} l_{t}}{\partial \boldsymbol{\xi}_{1} \partial \alpha} & \frac{\partial^{2} l_{t}}{\partial \alpha^{2}}
\end{array}\right]
$$

Diferenciando (5.62) em relação a $\boldsymbol{\xi}_{1}$ tem-se

$$
\frac{\partial^{2} l_{t}}{\partial \boldsymbol{\xi}_{1} \partial \boldsymbol{\xi}_{1}^{\prime}}=\frac{\partial^{2} l_{t}}{\partial \sigma_{t}^{2}} \frac{\partial \sigma_{t}}{\partial \boldsymbol{\xi}_{1}} \frac{\partial \sigma_{t}}{\partial \boldsymbol{\xi}_{1}^{\prime}}+\frac{\partial l_{t}}{\partial \sigma_{t}} \frac{\partial^{2} \sigma_{t}}{\partial \boldsymbol{\xi}_{1} \partial \boldsymbol{\xi}_{1}^{\prime}},
$$

em que

$$
\begin{aligned}
\frac{\partial^{2} \sigma_{t}}{\partial \boldsymbol{\xi}_{1} \partial \boldsymbol{\xi}_{1}^{\prime}}= & \frac{\partial \boldsymbol{z}_{t}}{\partial \boldsymbol{\xi}_{1}^{\prime}}+\frac{\partial \sigma_{t-1}}{\partial \boldsymbol{\xi}_{1}} \frac{\partial B_{1}}{\partial \boldsymbol{\xi}_{1}^{\prime}}+B_{1} \frac{\partial^{2} \sigma_{t-1}}{\partial \boldsymbol{\xi}_{1} \partial \boldsymbol{\xi}_{1}^{\prime}}+ \\
& +\left(\frac{\partial \sigma_{t-1}}{\partial \boldsymbol{\xi}_{1}} \frac{\partial A_{1}}{\partial \boldsymbol{\xi}_{1}^{\prime}}+A_{1} \frac{\partial^{2} \sigma_{t-1}}{\partial \boldsymbol{\xi}_{1} \partial \boldsymbol{\xi}_{1}^{\prime}}\right)\left(\frac{\partial^{2} l_{t-1}}{\partial \sigma_{t-1}^{2}}+\frac{\partial l_{t-1}}{\partial \sigma_{t-1}} \frac{\partial \mathcal{I}_{t}^{-1}}{\partial \sigma_{t-1}}\right)+ \\
& +A_{1} \frac{\partial \sigma_{t-1}}{\partial \boldsymbol{\xi}_{1}} \frac{\partial \sigma_{t-1}}{\partial \boldsymbol{\xi}_{1}^{\prime}}\left(\frac{\partial^{3} l_{t-1}}{\partial \sigma_{t-1}^{3}} \mathcal{I}_{t}^{-1}+2 \frac{\partial^{2} l_{t-1}}{\partial \sigma_{t-1}^{2}} \frac{\partial \mathcal{I}_{t}^{-1}}{\partial \sigma_{t-1}}+\frac{\partial l_{t-1}}{\partial \sigma_{t-1}} \frac{\partial^{2} \mathcal{I}_{t}^{-1}}{\partial \sigma_{t-1}^{2}}\right)
\end{aligned}
$$

é obtida diferenciando (5.65) em relação a $\boldsymbol{\xi}_{1}$. Considera-se $\partial^{2} \sigma_{t} / \partial \boldsymbol{\xi}_{1} \partial \boldsymbol{\xi}_{1}^{\prime}=0$ para $t \leq 0$.

Diferenciando (5.66) com relação a $\boldsymbol{\xi}_{1}$ tem-se

$$
\frac{\partial^{2} l_{t}}{\partial \boldsymbol{\xi}_{1} \partial \alpha}=\frac{1}{f^{*}\left(\frac{y_{t}}{\gamma\left(\sigma_{t}\right)}\right)^{2}}\left(\frac{\partial^{2} f^{*}\left(\frac{y_{t}}{\gamma\left(\sigma_{t}\right)}\right)}{\partial \alpha \partial \frac{y_{t}}{\gamma\left(\sigma_{t}\right)}} f^{*}\left(\frac{y_{t}}{\gamma\left(\sigma_{t}\right)}\right)^{2}-\frac{\partial f^{*}\left(\frac{y_{t}}{\gamma\left(\sigma_{t}\right)}\right)}{\partial \alpha} \frac{\partial f^{*}\left(\frac{y_{t}}{\gamma\left(\sigma_{t}\right)}\right)}{\partial \frac{y_{t}}{\gamma\left(\sigma_{t}\right)}}\right) \frac{\partial \frac{y_{t}}{\gamma\left(\sigma_{t}\right)}}{\partial \gamma\left(\sigma_{t}\right)} \frac{\partial \gamma\left(\sigma_{t}\right)}{\partial \sigma_{t}} \frac{\partial \sigma_{t}}{\partial \boldsymbol{\xi}_{1}} .
$$

Finalmente, diferenciando (5.66) com relação a $\alpha$ tem-se

$$
\frac{\partial^{2} l_{t}}{\partial^{2} \alpha}=\frac{\partial f^{*}\left(\frac{y_{t}}{\gamma\left(\sigma_{t}\right)}\right)}{\partial \alpha}\left(-\frac{1}{f^{*}\left(\frac{y_{t}}{\gamma\left(\sigma_{t}\right)}\right)^{2}} \frac{\partial f^{*}\left(\frac{y_{t}}{\gamma\left(\sigma_{t}\right)}\right)}{\partial \alpha}\right)+\frac{1}{f^{*}\left(\frac{y_{t}}{\gamma\left(\sigma_{t}\right)}\right)} \frac{\partial^{2} f^{*}\left(\frac{y_{t}}{\gamma\left(\sigma_{t}\right)}\right)}{\partial \alpha^{2}} .
$$

Logo, para o cálculo das expressões envolvidas no processo de estimação, as seguintes quanti- 
dades são necessárias:

$$
\frac{\partial l_{t}}{\partial \sigma_{t}}, \quad \frac{\partial^{2} l_{t}}{\partial \sigma_{t}^{2}}, \quad \frac{\partial^{3} l_{t}}{\partial \sigma_{t}^{3}}, \quad f^{*}(x), \quad \frac{\partial f^{*}(x)}{\partial \alpha} \quad \frac{\partial^{2} f^{*}(x)}{\partial \alpha^{2}} \quad \frac{\partial^{2} f^{*}(x)}{\partial \alpha \partial x} \quad \mathcal{I}_{t}, \quad \frac{\partial \mathcal{I}_{t}}{\partial \sigma_{t}}, \quad \frac{\partial^{2} \mathcal{I}_{t}}{\partial \sigma_{t}^{2}}
$$

O cálculo de todas as quantidades está descrito no Capítulo 4.

A matriz de informação de Fisher em relação a $\boldsymbol{\xi}$, dada por

$$
\mathcal{I}(\boldsymbol{\xi})=-\mathbb{E}\left[\frac{\partial^{2} L(\boldsymbol{\xi})}{\partial \boldsymbol{\xi} \partial \boldsymbol{\xi}^{\prime}}\right]=-\sum_{t=1}^{T} \mathbb{E}\left[\frac{\partial^{2} l_{t}(\boldsymbol{\xi})}{\partial \boldsymbol{\xi} \partial \boldsymbol{\xi}^{\prime}}\right],
$$

é a matriz de variâncias e covariâncias do estimador de máxima verossimilhança de $\boldsymbol{\xi}$. Como em geral seu cálculo é complicado, o procedimento mais utilizado é aproximá-la pelo seu valor amostral

$$
\hat{\mathcal{I}}(\boldsymbol{\xi})=-\frac{1}{T} \sum_{t=1}^{T} \frac{\partial^{2} l_{t}(\hat{\boldsymbol{\xi}})}{\partial \hat{\boldsymbol{\xi}} \partial \hat{\boldsymbol{\xi}}^{\prime}}=\frac{1}{T} \boldsymbol{H}(\hat{\boldsymbol{\xi}}) .
$$

Logo, tal procedimento para encontrar os erros padrão das componentes do vetor $\hat{\boldsymbol{\xi}}$ baseia-se em utilizar a matriz $\boldsymbol{H}^{-1}(\hat{\boldsymbol{\xi}})$, ou seja, a inversa da matriz Hessiana na última iteração do algoritmo de estimação (Rao, 1973).

O programa escrito em $R$ para a estimação dos parâmetros do modelo GAS com distribuição estável encontra-se na Seção A.3 do Apêndice A.

\subsubsection{Identificação do modelo}

Considere o modelo $\operatorname{GARCH}(p, q)$ com a equação de evolução da variância condicional dada em (5.2). Se $\varsigma_{t}$ for definido por $\varsigma_{t}=r_{t}^{2}-\sigma_{t}$, substituindo em (5.2), tem-se

$$
y_{t}^{2}=\omega+\sum_{i=1}^{m}\left(A_{i}+B_{i}\right) y_{t-i}^{2}+\nu_{t}-\sum_{j=1}^{q} B_{j} \varsigma_{t-j},
$$

em que $m=\max (p, q)$. Ou seja, $y_{t}^{2}$ segue um modelo $\operatorname{ARMA}(m, q)$. Observe que $\varsigma_{t}$ não é, em geral, ruído branco, e sim uma diferença martingal. Para mais detalhes, ver Morettin (2011).

Dessa forma, pode-se adotar um procedimento similar ao adotado para processos ARMA para avaliar se um conjunto de dados segue um processo GARCH e analisar sua função de autocorrelação amostral.

Um processo ARMA estacionário $x_{t}$ tipicamente tem os valores da função de autocorrelação entre $x_{t}$ e $x_{t+h}$ decaindo exponencialmente à medida de $h$ aumenta. Em diversas aplicações, é difícil determinar a ordem do modelo somente analisando a função de autocorrelação amostral. Mas, se os valores são substancialmente diferentes de zero, é um indício de que um modelo ARMA pode ser adequado.

Em um processo GARCH, a função de autocorrelação de $y_{t}^{2}$ em geral apresenta valores próximos de zero, mas significativos, e decaem conforme $h$ aumenta. Por isso, se a função de autocorrelação amostral de uma série exibir tal comportamento, é um indício de que um modelo GARCH é apropriado, apesar de na prática ser difícil identificar a ordem do modelo por esse procedimento.

Devido ao fato do modelo GARCH gaussiano ser um caso particular de modelo GAS com distribuição estável, pode-se pensar em um procedimento similar para identificação desse último tipo de processo. No entanto, há dois aspectos que devem ser levados em consideração:

- pelo fato de $y_{t}^{2}$ não aparecer explicitamente na expressão da evolução de $\sigma_{t}$, é difícil obter uma expressão analítica para sua evolução no caso geral do modelo GAS estável; e

- a função de autocorrelação não está definida no caso de variáveis aleatórias com distribuição estável com $0<\alpha<2$, simplesmente porque o segundo momento não existe. 
Uma alternativa é a análise da função de codiferença, apresentada na Seção 3.6. Para um processo $\left\{y_{t}\right\}$, a função de codiferença é definida por

$$
\tau_{y_{t}, y_{t+h}}=\tau_{y}(h)=\ln \mathbb{E}\left[e^{i\left(y_{t+h}-y_{t}\right)}\right]-\ln \mathbb{E}\left[e^{i y_{t}}\right]-\ln \mathbb{E}\left[e^{-i y_{t+h}}\right] .
$$

No caso gaussiano, a função de codiferença é a função de autocovariância. A função de codiferença normalizada, dada por

$$
\tau_{y}(h)^{*}=\frac{\tau_{y}(h)}{\tau_{y}(0)}
$$

é uma generalização da função de autocorrelação.

O estimador para a codiferença para uma série de tamanho $T$, proposto por Rosadi e Deistler (2011), é dado por

$$
\hat{\tau}_{y}(h)=\sqrt{\frac{T-h}{T}}\left[\ln \left(\frac{1}{T-h} \sum_{t=1}^{T-h} e^{i\left(y_{t+h}-y_{t}\right)}\right)-\ln \left(\frac{1}{T-h} \sum_{t=1}^{T} e^{i y_{t+h}}\right)-\ln \left(\frac{1}{T-h} \sum_{t=1}^{T} e^{-i y_{t}}\right)\right] .
$$

Suponha que deseja-se calcular a função de codiferença $\tau_{y_{t}, y_{t+h}}$ entre $y_{t}$ e $y_{t+h}$. Para o cálculo da quantidade $\mathbb{E}\left[e^{i y_{t}}\right]$, e sabendo-se que $y_{t}=\sqrt{\sigma_{t}} \epsilon_{t} \sim S_{\alpha}\left(0,0, \sqrt{\sigma_{t} / 2}\right)$,

$$
\mathbb{E}\left[e^{i y_{t}}\right]=\mathbb{E}\left[e^{i \sqrt{\sigma_{t}} \epsilon_{t}}\right]=\mathbb{E}\left[\mathbb{E}\left[e^{i \sqrt{\sigma_{t}} \epsilon_{t}}\right] \mid y_{1: t-1}\right]=\mathbb{E}\left[e^{-\left(\frac{\sqrt{\sigma_{t}}}{\sqrt{2}}\right)^{\alpha}}\right]
$$

Conforme já mencionado, no caso do modelo $\operatorname{GAS}(1,1)$, por substituições recursivas, $\sigma_{t}$ pode ser escrito como

$$
\sigma_{t}=\omega \sum_{i=0}^{\infty} B_{1}^{i-1}+A_{1} \sum_{i=1}^{\infty} B_{1}^{i-1} s_{t-i} .
$$

Para o cálculo de $\mathbb{E}\left[e^{-\left(\frac{\sqrt{\sigma_{t}}}{\sqrt{2}}\right)^{\alpha}}\right]$, portanto, é preciso da distribuição de ${\sqrt{\sigma_{t}}}^{\alpha}$, que, por sua vez, depende de $s_{t}$, que é o score ponderado pela inversa da informação de Fisher. Tal distribuição, pela complexidade envolvida, provavelmente é inacessível analiticamente e só pode ser obtida por métodos de simulação.

Por isso, nesta Seção, alguns cenários de modelos GAS serão simulados e suas funções de codiferença serão apresentadas.

Foram geradas séries seguindo processos GAS com distribuições estáveis de tamanho 1.000.000, para que se observe um comportamento bem próximo do teórico. De acordo com Frain (2009), séries financeiras que apresentem distribuição estável possuem valores de $\alpha$ entre 1,5 e 1,8. Aqui, foi considerado $\alpha=1,7$. O comportamento para outros valores de $\alpha$ é semelhante.

A função de autocorrelação, conforme já mencionado, não está definida para processos estáveis. No entanto, na prática, não se conhece o processo gerador dos dados, e o cálculo da função de autocorrelação amostral da série ao quadrado é uma das primeiras ferramentas para analisar seu comportamento.

Por isso, para fins de ilustração, a Figura 5.6 apresenta a função de autocorrelação amostral do quadrado de uma série de tamanho 1.000 .000 gerada de acordo com um processo GAS estável com $\alpha=1,7, \omega=0,1, A_{1}=0,1$ e $B_{1}=0,9$. As autocorrelações são praticamente nulas, comportamento esperado também em um processo GARCH (Morettin, 2011). Esse padrão se observa para quaisquer outros valores dos parâmetros testados nas simulações a seguir, comprovando na prática que a função de autocorrelação, que não está definida para o processo, não é adequada para identificação do modelo.

Na Figura 5.7, são apresentadas as funções de codiferença normalizada amostrais (parte real) do quadrado das séries para diversos cenários, com $\alpha=1,7$ e $\omega=0,5$. Consideram-se valores diversos de $A_{1}$ e $B_{1}$, seguindo condições para positividade de $\sigma_{t}$ e estacionariedade, determinadas nas Subseções 5.2.2 e 5.2.3. Os valores das codiferenças variam de acordo com os valores dos parâmetros, 


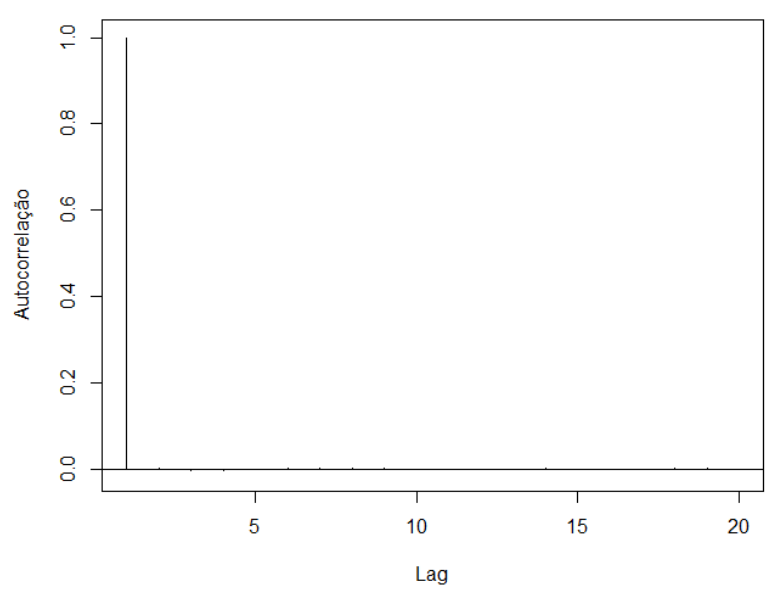

Figura 5.6: Função de autocorrelação amostral para o quadrado de um processo GAS estável gerado com $\alpha=1,7, \omega=0,1, A_{1}=0,1$ e $B_{1}=0,9$

mas em geral exibem o cenário de decaimento exponencial.

Na Figura 5.8, o cenário é o mesmo, com a única mudança para $\omega=2$. Observa-se que os valores das codiferenças são notavelmente maiores em comparação com as simulações correspondentes da Figura anterior. Por sua vez, se $\omega=0,01$, conforme apresentado na Figura 5.9, os valores das codiferenças na maioria dos cenários são muito próximos de zero. Portanto, dependendo dos valores dos parâmetros, é possível que se observem codiferenças nulas, e ainda assim um modelo GAS com distribuição estável seja adequado.

Os resultados apresentados das simulações têm por objetivo fornecer evidência de que a análise da função de codiferença amostral pode ser útil nos casos em que um modelo GAS com distribuição estável é um modelo viável quando se trabalha com séries reais. $\mathrm{Na}$ análise de séries financeiras, o estudo da função de autocorrelação amostral da série e da série ao quadrado pode indicar que um modelo GARCH pode ser adequado. Mas é uma ferramenta exploratória, e através dela não se detecta a ordem do modelo GARCH e tampouco as estimativas dos parâmetros. A função de codiferença pode ser utilizada de forma análoga em modelos GAS com distribuição estável, no sentido de, em uma análise exploratória, indicar que o ajuste de tal modelo faz sentido baseado na estrutura de dependência dos dados. 


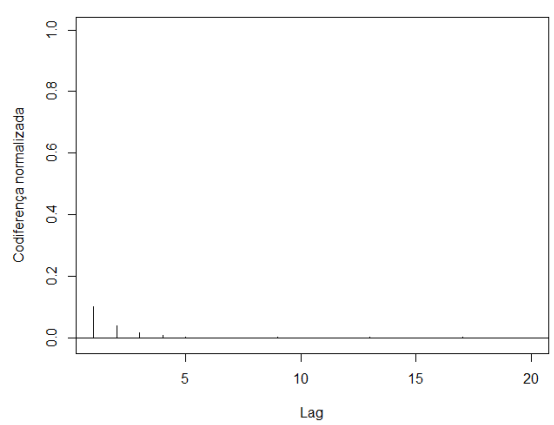

(a) $\omega=0,5, A_{1}=0,2, B_{1}=0,4$

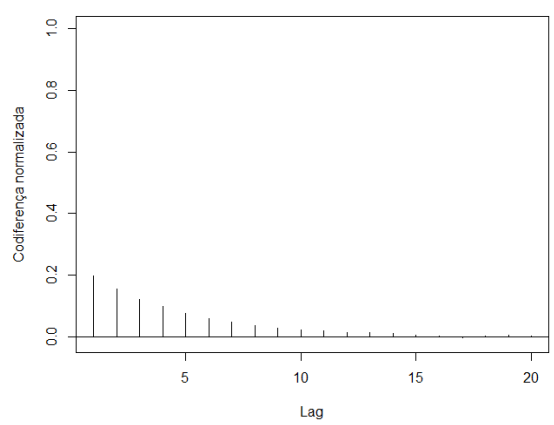

(c) $\omega=0,5, A_{1}=0,2, B_{1}=0,8$

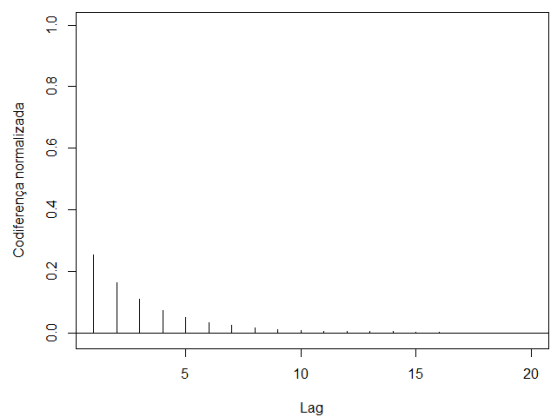

(e) $\omega=0,5, A_{1}=0,4, B_{1}=0,8$

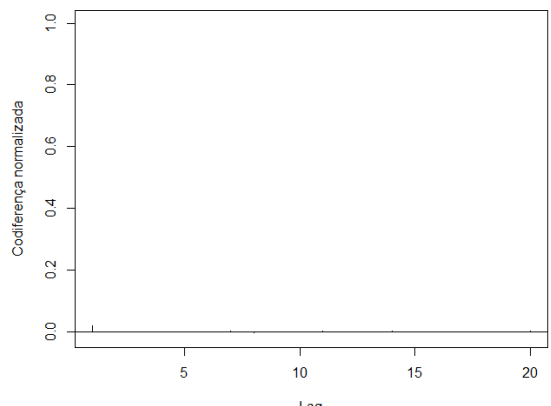

(g) $\omega=0,5, A_{1}=0,05, B_{1}=0,1$

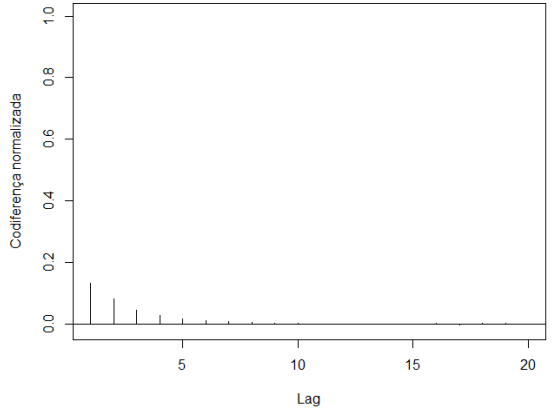

(b) $\omega=0,5, A_{1}=0,2, B_{1}=0,6$

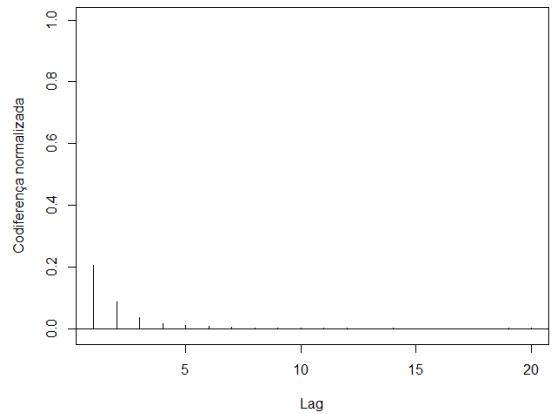

(d) $\omega=0,5, A_{1}=0,4, B_{1}=0,6$

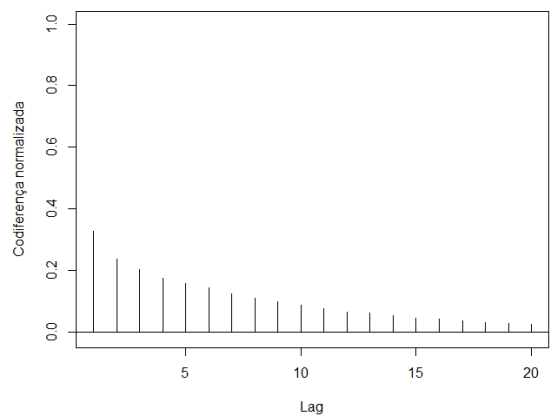

(f) $\omega=0,5, A_{1}=0,6, B_{1}=0,8$

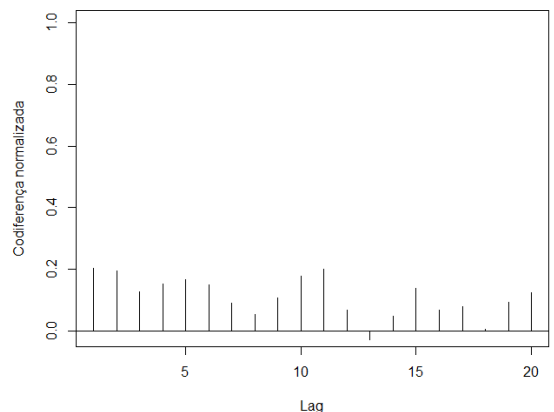

(h) $\omega=0,5, A_{1}=0,05, B_{1}=0,95$

Figura 5.7: Funções de codiferença normalizada amostrais do quadrado de simulações de processos GAS estáveis com $\alpha=1,7$ e $\omega=0,5$ 


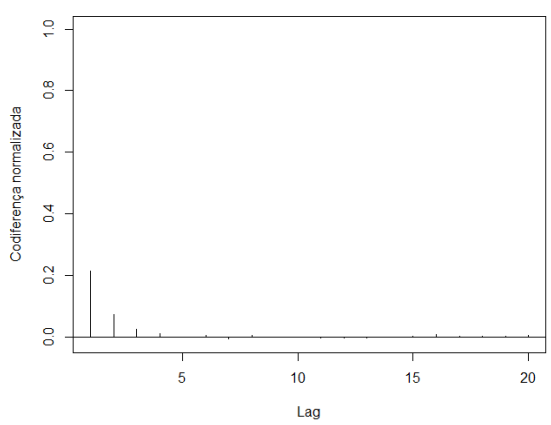

(a) $\omega=2, A_{1}=0,2, B_{1}=0,4$

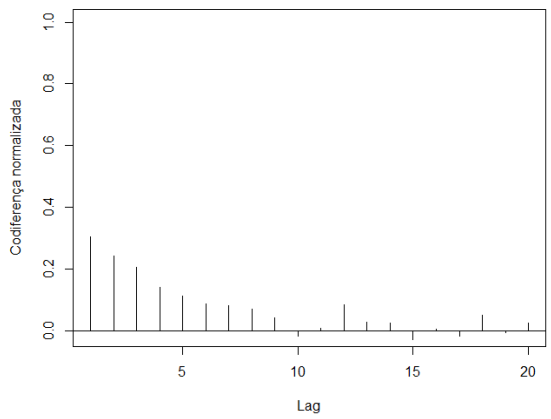

(c) $\omega=2, A_{1}=0,2, B_{1}=0,8$

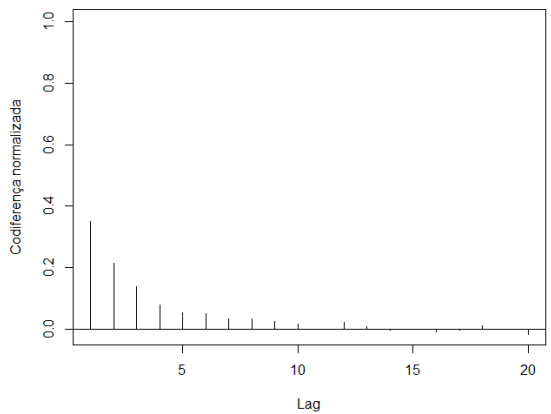

(e) $\omega=2, A_{1}=0,4, B_{1}=0,8$

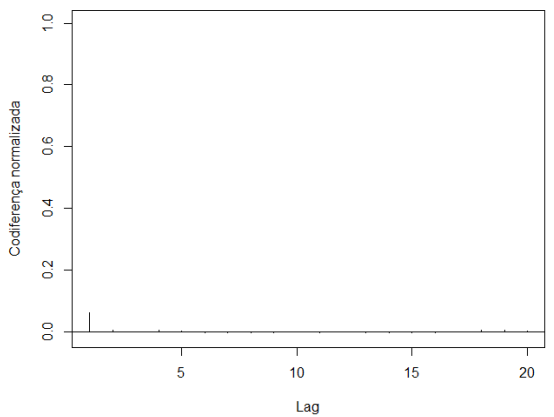

(g) $\omega=2, A_{1}=0,05, B_{1}=0,1$

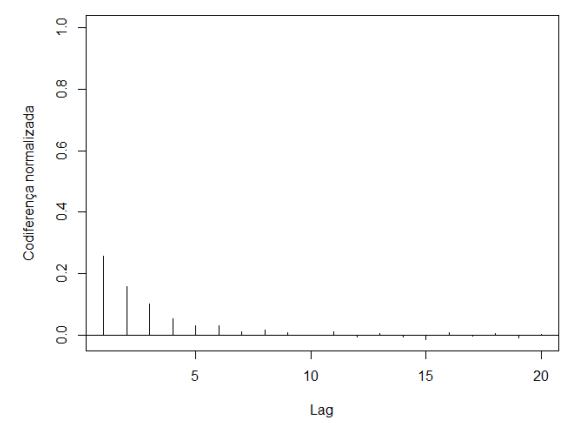

(b) $\omega=2, A_{1}=0,2, B_{1}=0,6$

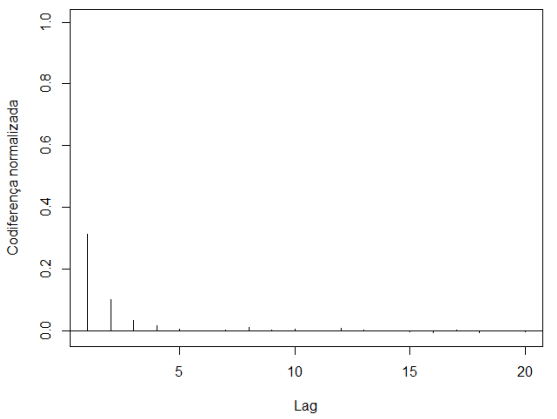

(d) $\omega=2, A_{1}=0,4, B_{1}=0,6$

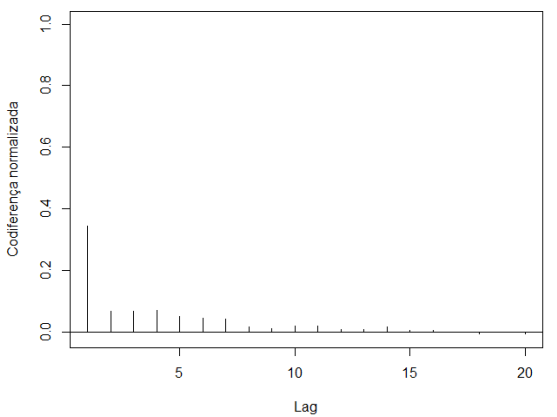

(f) $\omega=2, A_{1}=0,6, B_{1}=0,8$

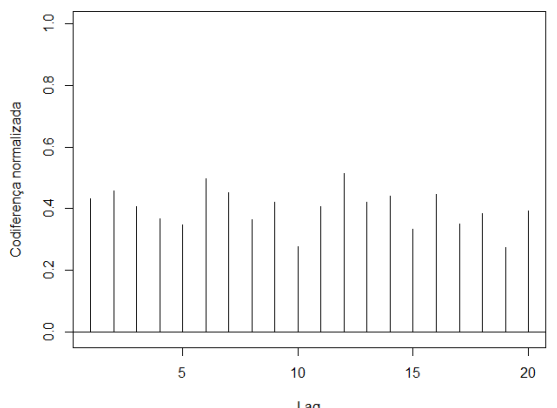

(h) $\omega=2, A_{1}=0,05, B_{1}=0,95$

Figura 5.8: Funções de codiferença normalizada amostrais de simulações de processos GAS estáveis com $\alpha=1,7$ e $\omega=2$ 


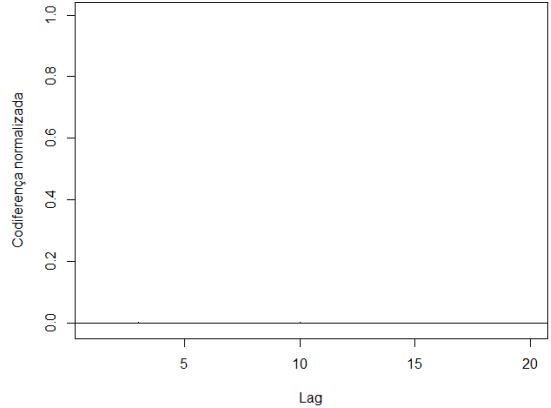

(a) $\omega=0,001, A_{1}=0,2, B_{1}=0,4$

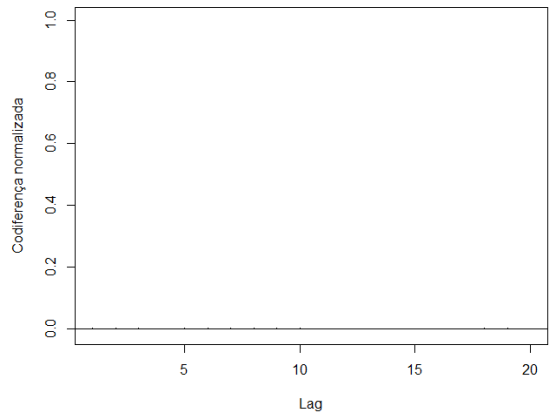

(c) $\omega=0,001, A_{1}=0,2, B_{1}=0,8$

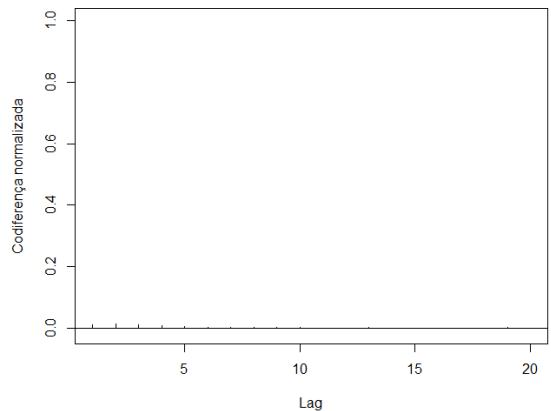

(e) $\omega=0,001, A_{1}=0,4, B_{1}=0,8$

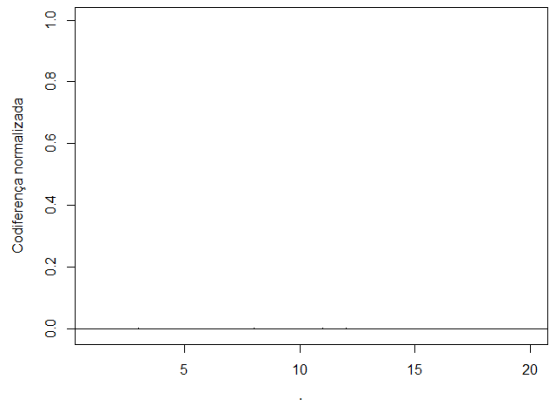

(g) $\omega=0,001, A_{1}=0,05, B_{1}=0,1$

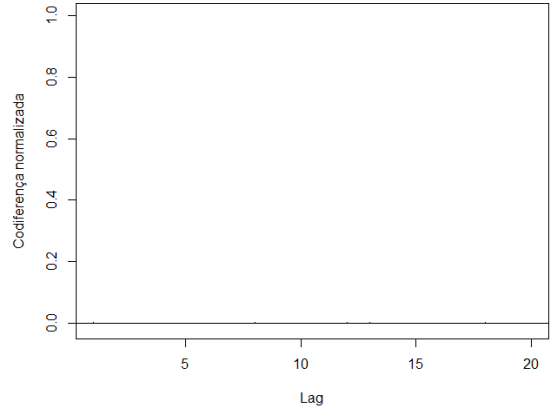

(b) $\omega=0,001, A_{1}=0,2, B_{1}=0,6$

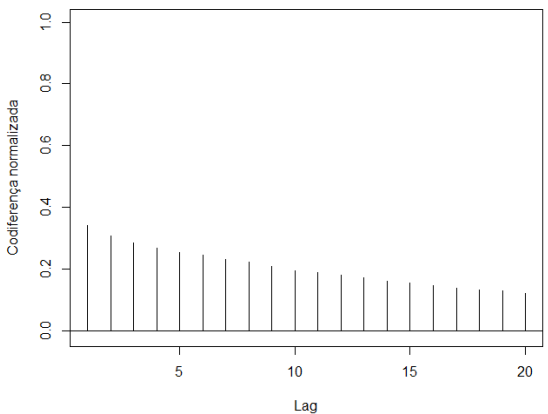

(d) $\omega=0,001, A_{1}=0,4, B_{1}=0,6$

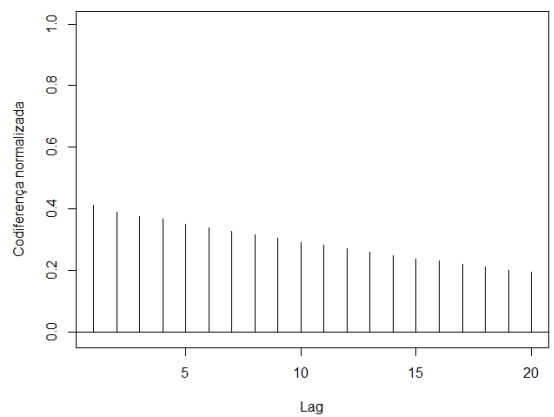

(f) $\omega=0,001, A_{1}=0,6, B_{1}=0,8$

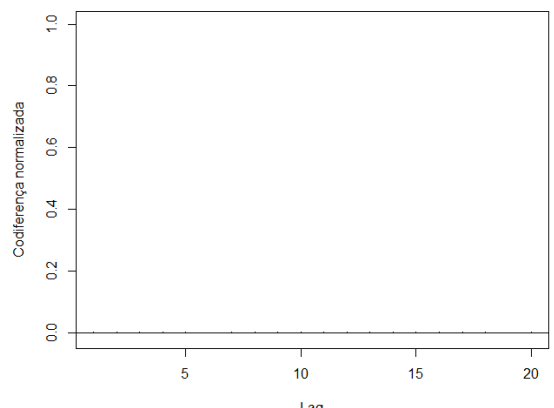

(h) $\omega=0,001, A_{1}=0,05, B_{1}=0,95$

Figura 5.9: Funções de codiferença normalizada amostrais do quadrado de simulações de processos GAS estáveis com $\alpha=1,7$ e $\omega=0,01$ 


\section{Capítulo 6}

\section{Simulações e aplicação}

\subsection{Simulações}

De acordo com Carnero et al. (2012), quando modelos GARCH são ajustados a dados reais, os resíduos em geral apresentam excesso de curtose que pode ser explicada, entre outras razões, pela presença de outliers. Na prática, modelos GARCH, na maioria das vezes, são ajustados sem levar em conta que pode haver observações geradas por um mecanismo estocástico diferente.

Ainda de acordo com os autores, os métodos de detecção de outliers apresentam diversos problemas, e uma alternativa é a utilização de métodos robustos; em particular a maximização da verossimilhança baseada em distribuições de caudas pesadas é uma proteção contra outliers no processo de estimação dos parâmetros do modelo.

Nesta Seção são apresentados estudos de simulação com o intuito de averiguar o desempenho do modelo proposto GAS com distribuição estável em comparação com os outros modelos citados no Capítulo 5 na presença de outliers.

Quando o processo é gerado por um modelo GARCH gaussiano, os resultados obtidos pelo modelo proposto obviamente são os mesmos quando se ajusta um modelo GARCH gaussiano, afinal os modelos são os mesmos quando $\alpha=2$. Por isso, para avaliar o desempenho do modelo proposto, será verificado seu comportamento quando os dados são gerados por um modelo GARCH gaussiano, mas com alguns outliers incorporados. Decidiu-se por tal procedimento após analisar o comportamento de séries geradas de acordo com o modelo proposto. Tais séries sugerem que o modelo poderia lidar bem com outliers.

Tal comportamento foi analisado após serem geradas séries de tamanho 1.000 do seguinte modelo $\operatorname{GAS}(1,1)$ :

$$
\begin{aligned}
y_{t} & =\sqrt{\sigma_{t}} \epsilon_{t}, \quad \epsilon_{t} \sim S_{\alpha}(0,0,1 / \sqrt{2}), \\
\sigma_{t} & =\omega+A_{1} \frac{\partial \ln f\left(y_{t-1} ; \sigma_{t-1}\right)}{\partial \sigma_{t-1}} \mathcal{I}_{t-1}^{-1}+B_{1} \sigma_{t-1},
\end{aligned}
$$

com $\omega=0,5, A_{1}=0,1$ e $B_{1}=0,6$, para diferentes valores de $\alpha$.

Para geração de $\epsilon_{t}$ com distribuição estável, foi utilizado o programa STABLE para R, que faz uso do método descrito na Seção 3.7.

Entre os valores de $\alpha$, um dos considerados foi $\alpha=2$, o que, como já visto, faz com que o modelo se torne o $\operatorname{GARCH}(1,1)$ gaussiano, com a seguinte estrutura:

$$
\begin{aligned}
& y_{t}=\sqrt{\sigma_{t}} \epsilon_{t}, \quad \epsilon_{t} \sim N(0,1), \\
& \sigma_{t}=\omega+A_{1} y_{t-1}^{2}+\left(B_{1}-A_{1}\right) \sigma_{t-1} .
\end{aligned}
$$

Também foram geradas séries do modelo GARCH com a mesma estrutura, mas com $\epsilon_{t}$ com distribuição $t$-Student padronizada, variando seus graus de liberdade.

Os gráficos das séries geradas encontram-se na Figura 6.1. Os gráficos estão na mesma escala, 


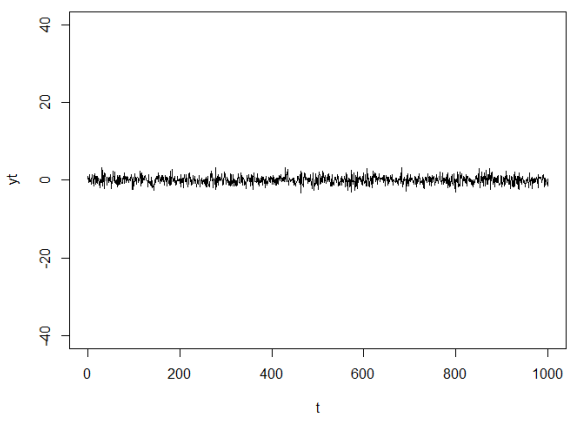

(a) $\operatorname{GARCH}(1,1)$ gaussiano

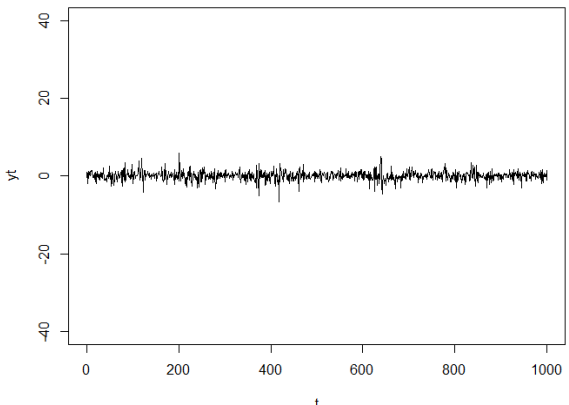

(c) $\operatorname{GARCH}(1,1) t$-Student, 4 g.l.

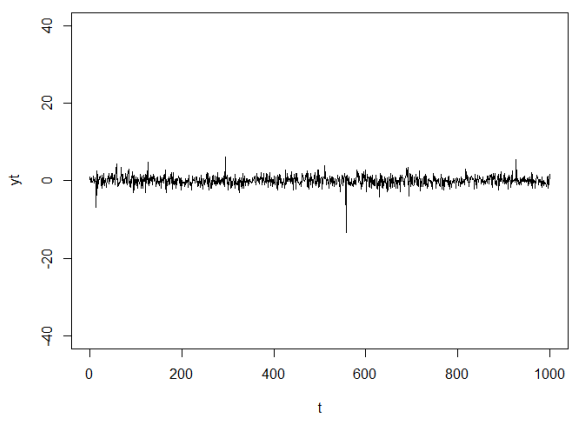

(e) GAS(1,1) estável, $\alpha=1,95$

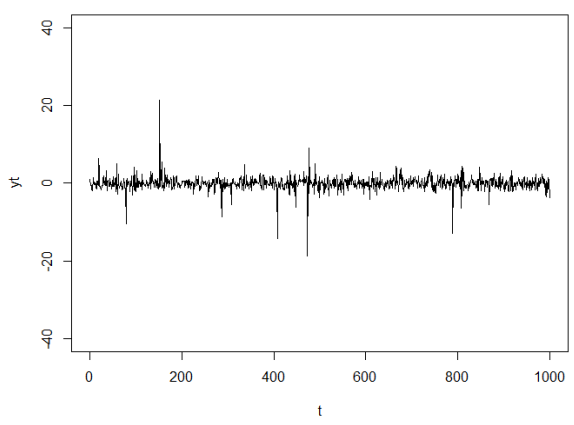

(g) GAS(1,1) estável, $\alpha=1,8$

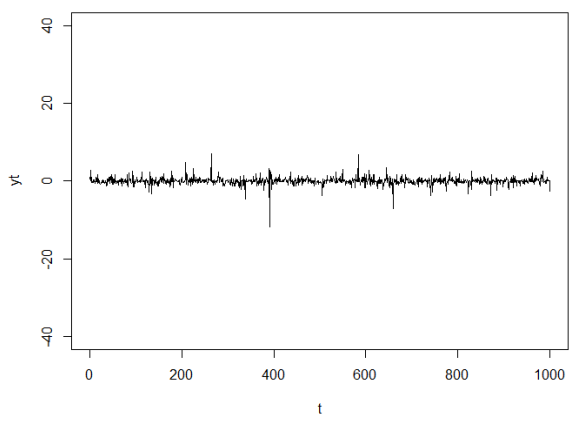

(b) $\operatorname{GARCH}(1,1) t$-Student, 2,5 g.l.

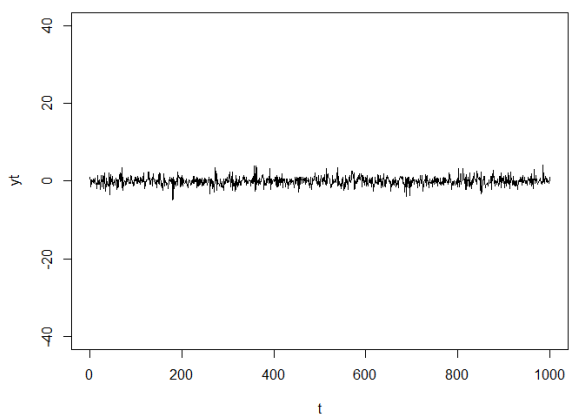

(d) $\operatorname{GARCH}(1,1) t$-Student, 7 g.l.

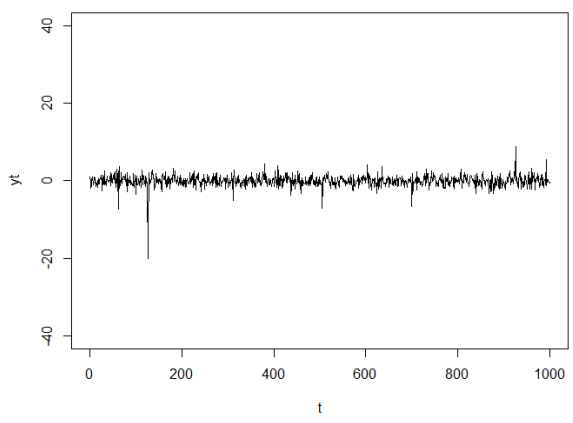

(f) $\operatorname{GAS}(1,1)$ estável, $\alpha=1,9$

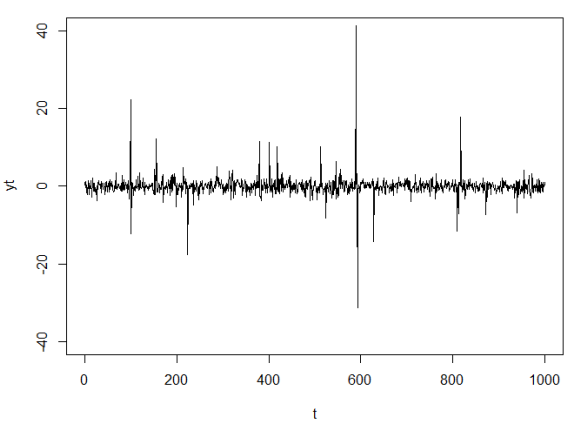

(h) GAS(1,1) estável, $\alpha=1,6$

Figura 6.1: Séries geradas por diferentes processos com $\omega=0,5, A_{1}=0,1$ e $B_{1}=0,6$ 
para facilitar as comparações.

Comparando com o processo GARCH gaussiano, nota-se ocorrência de valores que se destacam em termos de seus valores absolutos nos processos em que é utilizada a distribuição $t$-Student, especialmente quando o número de graus de liberdade é 2,5 , por causa do peso nas caudas. Também observa-se esse fenômeno nos modelos estáveis, com mais evidência quando o valor de $\alpha$ diminui. Quando $\alpha=1,95$, a distribuição estável é bem próxima da normal, mas já é possível observar claramente valores notáveis decorrentes dos pesos das caudas. Quando $\alpha=1,6$, observam-se vários valores que em um modelo gaussiano seriam totalmente aberrantes.

Os gráficos sugerem que, se alguns valores atípicos forem observados nas séries, modelos $t$ Student e modelos estáveis podem ser mais adequados em relação ao modelo gaussiano. A eficácia do modelo provavelmente dependerá da magnitude e da quantidade de valores extremos observados, até porque é clara a diferença de padrão de um modelo $t$-Student de cauda pesada $(2,5$ graus de liberdade) e de um modelo estável $\operatorname{com} \alpha=1,6$. Até uma faixa de magnitude, parece que os modelos $t$-Student podem ser suficientes para uma modelagem adequada. Se a magnitude for muito alta, aparentemente será necessário um modelo estável, com um valor de $\alpha$ adequado.

Considere um processo $\operatorname{GARCH}(1,1)$ contaminado por outliers de tamanho $\rho$ nos instantes de tempo $t_{1}, \ldots, t_{K}$ :

$$
y_{t}=y_{t}^{*}+\operatorname{sinal}\left(y_{t}^{*}\right) \rho I_{t}
$$

com

$$
y_{t}^{*}=\sqrt{\sigma_{t}} \epsilon_{t},
$$

em que $\epsilon_{t}$ é um ruído aleatório gaussiano, $I_{t}$ tem valor 1 se $t=t_{1}, \ldots, t_{K}$ e 0 caso contrário, e

$$
\sigma_{t}=\alpha_{0}+\alpha_{1} y_{t-1}^{* 2}+\beta_{1} \sigma_{t-1} .
$$

Baseado no estudo sugerido por Carnero et al. (2012), foi conduzido um experimento de Monte Carlo, em que foram geradas réplicas de modelos $\operatorname{GARCH}(1,1)$ gaussianos conforme descrito nas equações (6.5) a (6.7) para cada um dos conjuntos de valores de parâmetros:

- $\alpha_{0}=0,1, \alpha_{1}=0,1, \beta_{1}=0,8$;

- $\alpha_{0}=0,3, \alpha_{1}=0,15, \beta_{1}=0,55$; e

- $\alpha_{0}=0,5, \alpha_{1}=0,2, \beta_{1}=0,3$.

Lembremos mais uma vez que o $\operatorname{GARCH}(1,1)$ gaussiano é um caso particular do $\operatorname{GAS}(1,1)$, e a distribuição normal, por sua vez, é uma distribuição estável com $\alpha=2$. Para cada conjunto de parâmetros, as séries foram contaminadas por outliers de tamanhos $\rho=5,10$ e 15. Os valores dos parâmetros fazem com que a variância incondicional das séries seja unitária. Consequentemente, $\rho$ representa o tamanho do outlier em termos do número de desvios padrão.

O número de outliers considerado se divide nas seguintes configurações:

- Um outlier no instante $t=500$;

- Dois outliers consecutivos nos instantes $t=500$ e $t=501$;

- Dois outliers não-consecutivos nos instantes $t=333$ e $t=666$;

- Dois conjuntos não-consecutivos de dois outliers consecutivos, nos instantes $t=333$ e $t=334$, e $t=666$ e $t=667$;

- Três outliers não-consecutivos nos instantes $t=250, t=500$ e $t=750$; e

- Três conjuntos não-consecutivos de dois outliers consecutivos, nos instantes $t=250$ e $t=251$, $t=500$ e $t=501$, e $t=750$ e $t=751$. 
As combinações de valores de parâmetros e outliers resultam em 54 diferentes cenários. Para cada um dos cenários, foram geradas 1.000 réplicas de tamanho 1.000, para as quais cinco modelos foram ajustados.

Todos os ajustes foram feitos pelo software R. Para ajuste dos modelos GARCH gaussiano e $t$-Student, foi utilizado o pacote fGarch. Para o modelo GARCH estável, foi utilizado o pacote GEVStableGarch, que trabalha em conjunto com o programa STABLE. Para o modelo GAS $t$ Student foi utilizado o pacote GAS. Para o modelo GAS estável, foi elaborado um código em R para estimação dos parâmetros, de acordo com os cálculos exibidos nos Capítulos 4 e 5. O código está disponível nas Seções A.1 e A.2 do Apêndice A. Todos os modelos são da ordem $p=1$ e $q=1$.

A seleção de modelos para séries temporais é usualmente feita minimizando-se algum critério de informação. Aqui, a comparação é feita em termos da versão corrigida do Critério de Informação de Akaike (AICc) e do Critério de Informação Bayesiano (BIC). Quanto menor o valor dessas estatísticas, mais adequado é o modelo aos dados.

Definição 6.1.1 O AIC corrigido para um modelo com $r$ parâmetros ajustado em uma amostra de tamanho $n$ é dado pela seguinte expressão:

$$
A I C c=-2 L(\hat{\boldsymbol{\xi}})+\frac{2(r+1) n}{n-r-2},
$$

em que $L(\hat{\boldsymbol{\xi}})$ é a log-verossimilhança do modelo avaliada em $\hat{\boldsymbol{\xi}}$, o estimador do vetor de parâmetros estáticos $\boldsymbol{\xi}$.

Definição 6.1.2 O BIC para um modelo com r parâmetros ajustado em uma amostra de tamanho $n$ é dado pela seguinte expressão:

$$
B I C=-2 L(\hat{\boldsymbol{\xi}})+r \ln n .
$$

Para o cenário com $\alpha_{0}=0,1, \alpha_{1}=0,1, \beta_{1}=0,8$ e um outlier de tamanho $\rho=10$ no instante $t=500$, serão apresentados resultados mais detalhados a seguir. Para os outros cenários, serão

\begin{tabular}{|c|c|c|c|c|c|}
\hline \multirow[b]{2}{*}{ Estatística } & \multicolumn{5}{|c|}{$\mathrm{AICc}$} \\
\hline & $\begin{array}{c}\text { GARCH } \\
\text { gaussiano }\end{array}$ & $\begin{array}{c}\text { GARCH } \\
t \text {-Student }\end{array}$ & $\begin{array}{c}\text { GARCH } \\
\text { estável }\end{array}$ & $\begin{array}{c}G A S \\
t \text {-Student }\end{array}$ & $\begin{array}{c}\text { GAS } \\
\text { estável }\end{array}$ \\
\hline Mínimo & 2645,925 & 2601,967 & 2574,919 & 2591,909 & 2577,611 \\
\hline $1^{\circ}$ quartil & 2848,877 & 2791,077 & 2771,04 & 2785,006 & 2766,353 \\
\hline Mediana & 2902,075 & 2842,734 & 2821,947 & 2835,796 & 2815,653 \\
\hline Média & 2902,407 & 2846,773 & 2827,017 & 2840,035 & 2820,979 \\
\hline $3^{\circ}$ quartil & 2954,944 & 2902,343 & 2884,029 & 2895,798 & 2877,885 \\
\hline Máximo & 3140,375 & 3096,704 & 3074,64 & 3091,023 & 3071,898 \\
\hline \multirow[b]{2}{*}{ Estatistica } & \multicolumn{5}{|c|}{$\overline{\mathrm{BIC}}$} \\
\hline & $\begin{array}{c}\text { GARCH } \\
\text { gaussiano }\end{array}$ & $\begin{array}{c}\text { GARCH } \\
t \text {-Student }\end{array}$ & $\begin{array}{c}\text { GARCH } \\
\text { estável }\end{array}$ & $\begin{array}{c}G A S \\
t \text {-Student }\end{array}$ & $\begin{array}{c}\text { GAS } \\
\text { estável }\end{array}$ \\
\hline Mínimo & 2658,608 & 2619,548 & 2592,5 & 2609,49 & 2595,191 \\
\hline $1^{\circ}$ quartil & 2861,56 & 2808,658 & 2788,62 & 2802,587 & 2783,933 \\
\hline Mediana & 2914,758 & 2860,315 & 2839,528 & 2853,377 & 2833,233 \\
\hline Média & 2915,09 & 2864,353 & 2844,598 & 2857,616 & 2838,56 \\
\hline $3^{\circ}$ quartil & 2967,627 & 2919,924 & 2901,61 & 2913,379 & 2895,465 \\
\hline Máximo & 3153,058 & 3114,285 & 3092,221 & 3108,604 & 3089,479 \\
\hline
\end{tabular}
apresentados resumos em tabelas.

Tabela 6.1: Estatísticas das distribuições dos AICs corrigidos e dos BICs dos modelos ajustados para simulações de $\operatorname{GARCH}(1,1)$ gaussiano com $\alpha_{0}=0,1, \alpha_{1}=0,1, \beta_{1}=0,8$ e um outlier de tamanho $\rho=10$ 
A Tabela 6.1 exibe os comportamentos das distribuições do AIC corrigido e do BIC para cada um dos modelos, através das estatísticas descritivas. Em negrito, encontram-se os menores valores para a estatística correspondente entre os cinco modelos. A Figura 6.2 mostra as distribuições do AIC corrigido através de box-plots. O gráfico para as distribuições do BIC é semelhante, e por isso é omitido.

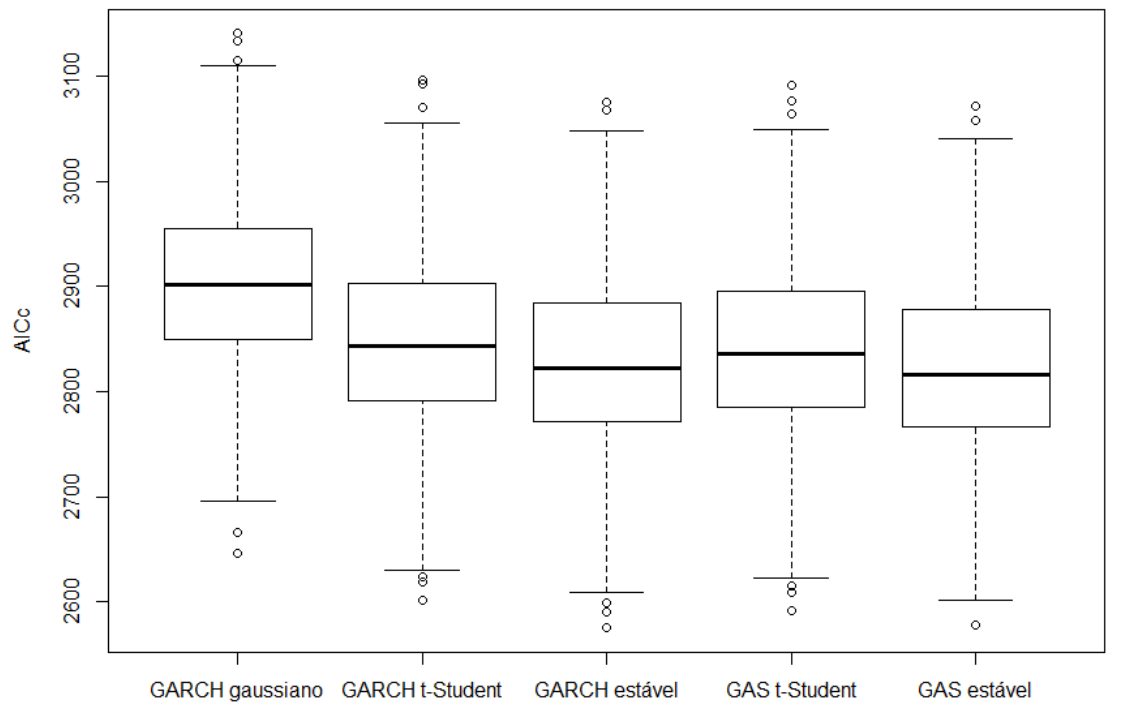

Figura 6.2: Box-plots das distribuições dos AICs corrigidos e dos BICs dos modelos ajustados para simulações de $\operatorname{GARCH}(1,1)$ gaussiano com $\alpha_{0}=0,1, \alpha_{1}=0,1, \beta_{1}=0,8$ e um outlier de tamanho $\rho=10$

Os resultados obtidos pelo modelo GARCH gaussiano são os piores em relação aos outros modelos. A presença de somente um outlier é suficiente para que o modelo não se adeque de maneira satisfatória aos dados, devido às caudas leves da normal. O modelo GARCH $t$-Student tem desempenho melhor, devido ao peso de suas caudas conseguirem lidar com o outlier. A mediana dos graus de liberdade é 9,92 , o que não confere muito peso às caudas, mas suficiente para um desempenho mais adequado que o do modelo gaussiano. O modelo GAS $t$-Student tem um desempenho ainda melhor, pois, como já explicado, devido à sua estrutura pode ser um modelo mais robusto que o GARCH. Mas ainda melhor que tais modelos são os que utilizam distribuições estáveis. Os modelos GARCH e GAS estáveis têm desempenhos semelhantes, com ligeira vantagem para o GAS em quase todas as estatísticas.

Em relação à verificação da hipótese da distribuição de probabilidade dos resíduos postulada pelos respectivos modelos, uma análise é feita em termos de $p$-valores de testes de qualidade de ajuste. Dois testes foram considerados: Anderson-Darling (AD) e Cramér-von Mises (CVM). A hipótese nula é de que os resíduos seguem a distribuição considerada no modelo em questão (normal, $t$-Student ou estável).

Teste de Anderson-Darling Para o cálculo da estatística $A^{2}$ do teste AD a uma série $\epsilon_{t}$, $t=1, \ldots, n$, o seguinte procedimento é utilizado:

- Faça a transformação $z_{t}=F\left(\epsilon_{t}\right)$, em que $F$ é a função de distribuição da distribuição para a qual deseja-se realizar o teste (no caso, normal, t-Student ou estável);

- Calcule a estatística $A^{2}$ da seguinte maneira:

$$
A^{2}=-n-\frac{1}{n} \sum_{t=1}^{n}(2 t-1)\left[\ln z_{t}+\ln \left(1-z_{n+1-t}\right)\right] .
$$


Teste de Cramér-von Mises O teste de Cramér-von Mises compara uma dada distribuição acumulada amostral empírica com a função distribuição $F(x)$ postulada. Dada uma série $\epsilon_{t}, t=$ $1, \ldots, n$, considere as estatísticas de ordem associadas $\epsilon_{t: n}, t=1, \ldots, n$. A estatística de teste é dada por

$$
C V M=\frac{1}{12 n}+\sum_{t=1}^{n}\left[\frac{2 t-1}{2 n}-F\left(\epsilon_{t: n}\right)\right]^{2} .
$$

Para avaliação da distribuição dos resíduos, a Tabela 6.2 apresenta os resultados referentes aos $p$-valores dos testes AD e CVM para os resíduos de cada modelo em relação ao ajuste da respectiva distribuição. Nota-se pelas estatísticas, especialmente do teste AD, que os modelos com distribuições $t$-Student e estável geram resíduos que se adequam satisfatoriamente às distribuições postuladas.

\begin{tabular}{|c|c|c|c|c|c|}
\hline \multirow{2}{*}{ Estatística } & \multicolumn{5}{|c|}{ Teste AD } \\
\cline { 2 - 6 } & $\begin{array}{c}\text { GARCH } \\
\text { gaussiano }\end{array}$ & $\begin{array}{c}\text { GARCH } \\
\text { t-Student }\end{array}$ & $\begin{array}{c}\text { GARCH } \\
\text { estável }\end{array}$ & $\begin{array}{c}\text { GAS } \\
\text { t-Student }\end{array}$ & $\begin{array}{c}\text { GAS } \\
\text { estável }\end{array}$ \\
\hline \hline \% acima de 0,05 & 57,6 & 90,9 & 96,0 & 100 & 96,0 \\
$1^{\circ}$ quartil & 0 & 0,206 & 0,322 & 0,499 & 0,29 \\
Mediana & 0,261 & 0,43 & 0,586 & 0,636 & 0,563 \\
$3^{\text {o quartil }}$ & 0,449 & 0,647 & 0,86 & 0,787 & 0,841 \\
\hline \hline & \multicolumn{5}{|c|}{ Teste CVM } \\
Estatística & GARCH & GARCH & GARCH & GAS & GAS \\
& gaussiano & t-Student & estável & t-Student & estável \\
\hline \hline \% acima de 0,05 & 100 & 89,9 & 94,9 & 100 & 94,9 \\
$1^{\circ}$ quartil & 0,307 & 0,213 & 0,299 & 0,568 & 0,266 \\
Mediana & 0,482 & 0,442 & 0,553 & 0,704 & 0,536 \\
$3^{\text {o quartil }}$ & 0,636 & 0,66 & 0,832 & 0,867 & 0,819 \\
\hline
\end{tabular}

Tabela 6.2: Estatísticas das distribuições dos p-valores dos testes AD e CVM para os resíduos dos modelos ajustados para simulações de $\operatorname{GARCH}(1,1)$ gaussiano com $\alpha_{0}=0,1, \alpha_{1}=0,1, \beta_{1}=0,8$ e um outlier de tamanho $\rho=10$

A Figura 6.3 traz a distribuição dos valores estimados para o parâmetro $\alpha$ do modelo GAS estável. Nota-se que há uma grande concentração de valores entre 1,97 e 1,99. Valores próximos de 2 indicam distribuições próximas à normal. Mas conferem suficiente peso às caudas para se adequar ao outlier de maneira mais satisfatória que a distribuição normal.

Também foi feita uma comparação com base nas previsões da volatilidade através de "janelas" móveis e reestimação dos modelos, de acordo com procedimento sugerido por Akgiray (1989). Inicialmente, os modelos são estimados com as primeiras $T-225=775$ observações, e previsões para $\sigma_{t}$ são geradas para as 15 observações seguintes. Isso resulta no primeiro conjunto de previsão. Para cada conjunto de previsões subsequentes, a amostra utilizada para estimação do modelo tem início e fim com 15 observações depois. Logo, o número de conjuntos de previsões gerado é 15 (225 dividido por 15$)$.

As previsões são calculadas de acordo com a estrutura de cada modelo, de acordo com o descrito nas equações (5.3), (5.8), (5.13) e (5.18).

A soma das previsões de $\sigma_{t}$ para cada conjunto de 15 observações é comparada com o valor correspondente amostral do período (que pode ser a variância, no caso dos modelos normal e $t$ Student, ou o parâmetro de escala, no caso dos modelos que utilizam distribuições estáveis, estimado por máxima verossimilhança) através do MAE (Mean Absolute Error). 


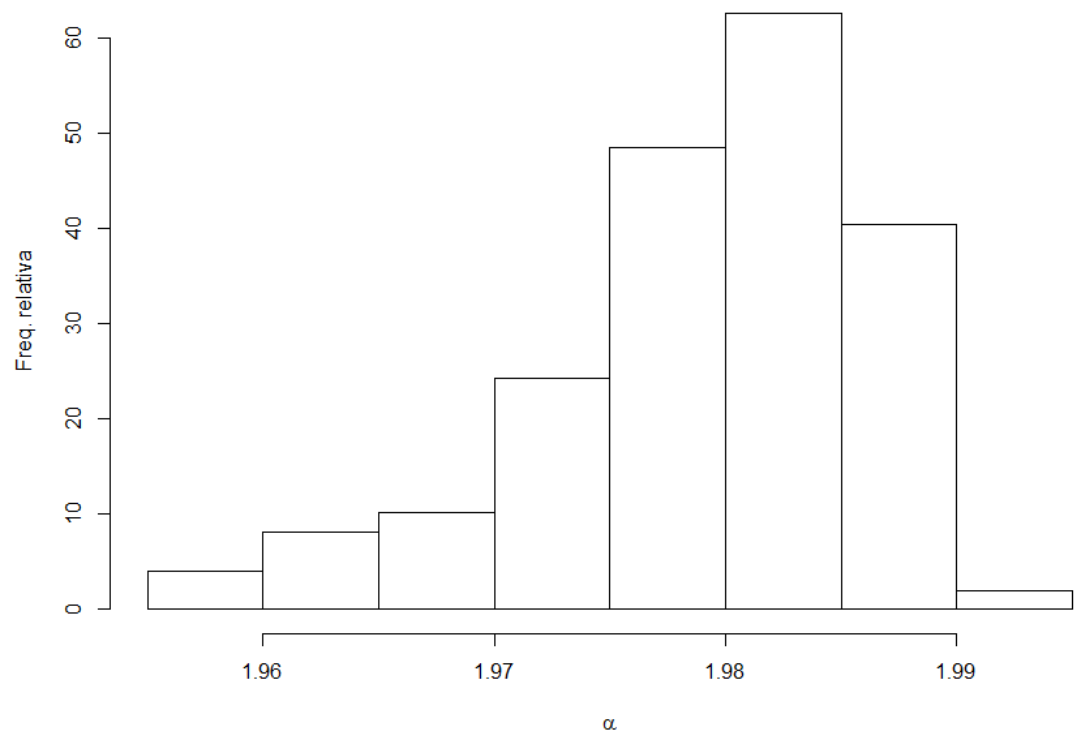

Figura 6.3: Histograma da distribuição dos valores estimados de $\alpha$ do modelo GAS(1,1) estável para simulações de $\operatorname{GARCH}(1,1)$ gaussiano com $\alpha_{0}=0,1, \alpha_{1}=0,1, \beta_{1}=0,8$ e um outlier de tamanho $\rho=10$

Definição 6.1.3 O MAE entre uma série $\left\{y_{t}^{(1)}, t=1, \ldots, n\right\}$ e uma série $\left\{y_{t}^{(2)}, t=1, \ldots, n\right\}$ é dado por

$$
\operatorname{MAE}\left(y^{(1)}, y^{(2)}\right)=\frac{1}{n} \sum_{t=1}^{n}\left|y_{t}^{(1)}-y_{t}^{(2)}\right|
$$

Os resultados são apresentados na Tabela 6.3. Em negrito, encontram-se os menores valores para a estatística correspondente entre os cinco modelos. Os modelos com melhor desempenho são o GAS t-Student e o GARCH e o GAS estáveis, com pequena vantagem para o GARCH.

\begin{tabular}{|c|c|c|c|c|c|}
\hline Estatística & $\begin{array}{c}\text { GARCH } \\
\text { gaussiano }\end{array}$ & $\begin{array}{c}\text { GARCH } \\
\text { t-Student }\end{array}$ & $\begin{array}{c}\text { GARCH } \\
\text { estável }\end{array}$ & $\begin{array}{c}\text { GAS } \\
\text { t-Student }\end{array}$ & $\begin{array}{c}\text { GAS } \\
\text { estável }\end{array}$ \\
\hline \hline Mínimo & 4,035 & 3,529 & 3,449 & $\mathbf{3 , 0 5 7}$ & 3,406 \\
$1^{\circ}$ quartil & 5,821 & 5,339 & 4,934 & 4,888 & $\mathbf{4 , 8 8 6}$ \\
Mediana & 7,195 & 6,714 & $\mathbf{6 , 0 9 5}$ & 6,159 & 6,165 \\
Média & 7,431 & 6,809 & $\mathbf{6 , 3 9 6}$ & 6,605 & 6,446 \\
$3^{\circ}$ quartil & 8,813 & 8,17 & $\mathbf{7 , 5 4 6}$ & 7,958 & 7,669 \\
Máximo & 16,256 & 14,622 & $\mathbf{1 3 , 7 3 4}$ & 16,987 & 14,524 \\
\hline
\end{tabular}

Tabela 6.3: Estatísticas das distribições dos MAEs para previsões da volatilidade para simulações de $\operatorname{GARCH}(1,1)$ gaussiano com $\alpha_{0}=0,1, \alpha_{1}=0,1, \beta_{1}=0,8$ e um outlier de tamanho $\rho=10$

Após a análise desse cenário em particular, prosseguimos com a análise dos outros cenários. Os resultados dos 54 cenários, em termos das medianas de algumas das estatísticas, estão apresentadas nas Tabelas 6.5 a 6.12 . Também são apresentadas as medianas dos valores estimados do número de graus de liberdade $\nu$, no caso de modelos com a distribuição $t$-Student, e do parâmetro característico $\alpha$, no caso de modelos com distribuição estável. 


\begin{tabular}{|c|c|c|c|c|c|}
\hline \multirow[b]{2}{*}{ Estatistica } & \multicolumn{5}{|c|}{ Um outlier, $t=500$} \\
\hline & $\begin{array}{c}\text { GARCH } \\
\text { gaussiano }\end{array}$ & $\begin{array}{c}\text { GARCH } \\
t \text {-Student }\end{array}$ & $\begin{array}{c}\text { GARCH } \\
\text { estável }\end{array}$ & $\begin{array}{c}G A S \\
t \text {-Student }\end{array}$ & $\begin{array}{c}\text { GAS } \\
\text { estável }\end{array}$ \\
\hline AIC & 11,853 & 2811,109 & 2806,335 & 2806,587 & 2800,939 \\
\hline alor & 0,901 & 0,379 & 0,662 & 0,786 & 0,619 \\
\hline MAE (pre & 6,255 & 6,48 & 5,956 & 5,859 & 5,91 \\
\hline t & - & 10 & 1,982 & 15,629 & 1,983 \\
\hline \multirow[b]{2}{*}{ Estatistica } & \multicolumn{5}{|c|}{ Dois outliers, $t=500,501$} \\
\hline & $\begin{array}{c}\text { GARCH } \\
\text { gaussiano }\end{array}$ & $\begin{array}{c}\text { GARCH } \\
t \text {-Student }\end{array}$ & $\begin{array}{c}\text { GARCH } \\
\text { estável }\end{array}$ & $\begin{array}{c}G A S \\
t \text {-Student }\end{array}$ & $\begin{array}{c}\text { GAS } \\
\text { estável }\end{array}$ \\
\hline$\overline{\mathrm{AICc}}$ & 2843,35 & 2842,649 & 2838,059 & 2836,228 & 2836,775 \\
\hline$p$-valor & 0,799 & 0,381 & 0,555 & 0,519 & 0,514 \\
\hline MAE (prev & 6,087 & 6,426 & 5,904 & 5,924 & 6,06 \\
\hline$\nu \backslash \alpha$ & - & 10 & 1,974 & 12,216 & 1,968 \\
\hline \multirow[b]{2}{*}{ Estatística } & \multicolumn{5}{|c|}{ 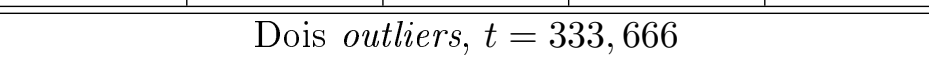 } \\
\hline & $\begin{array}{c}\text { GARCH } \\
\text { gaussiano }\end{array}$ & $\begin{array}{c}\text { GARCH } \\
t \text {-Student }\end{array}$ & $\begin{array}{c}\text { GARCH } \\
\text { estável }\end{array}$ & $\begin{array}{c}G A S \\
t \text {-Student }\end{array}$ & $\begin{array}{c}\text { GAS } \\
\text { estável }\end{array}$ \\
\hline & 6 & & 2853,428 & 2854,80 & 845,661 \\
\hline$p$-valor & 09 & 0 & 0,585 & 0,711 & 0,568 \\
\hline MAE (prev & 5,832 & 6,096 & 5,367 & 5,499 & 5,397 \\
\hline$\nu \backslash \alpha$ & - & 10 & 1,969 & 12,925 & 1,971 \\
\hline \multirow[b]{2}{*}{ Estatistica } & \multicolumn{5}{|c|}{ Quatro outliers, $t=333,334,666,667$} \\
\hline & $\begin{array}{c}\text { GARCH } \\
\text { gaussiano }\end{array}$ & $\begin{array}{c}\text { GARCH } \\
\text { t-Student }\end{array}$ & $\begin{array}{c}\text { GARCH } \\
\text { estável }\end{array}$ & $\begin{array}{c}G A S \\
t \text {-Student }\end{array}$ & $\begin{array}{c}\text { GAS } \\
\text { estável }\end{array}$ \\
\hline$\overline{\mathrm{AICc}}$ & 2888,484 & $\overline{28}$ & 2875,126 & 2873,939 & 2873,701 \\
\hline lor $(A$ & 0,787 & 0,563 & 0,778 & 0,38 & 0,811 \\
\hline (pres & 7,019 & 7,197 & 6,205 & 5,744 & 6,314 \\
\hline$\nu \backslash \alpha$ & 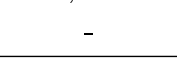 & 10 & 1,961 & 8,758 & 1,948 \\
\hline \multirow[b]{2}{*}{ Estatistica } & \multicolumn{5}{|c|}{ Três outliers, $t=250,500,750$} \\
\hline & $\begin{array}{c}\text { GARCH } \\
\text { gaussiano }\end{array}$ & $\begin{array}{c}\text { GARCH } \\
t \text {-Student }\end{array}$ & $\begin{array}{c}\text { GARCH } \\
\text { estável }\end{array}$ & $\begin{array}{c}G A S \\
t \text {-Student }\end{array}$ & $\begin{array}{c}\text { GAS } \\
\text { estável }\end{array}$ \\
\hline$\overline{\mathrm{AICc}}$ & 2899,308 & 2886,995 & 2877,151 & 2882,466 & 2871,779 \\
\hline alor ( & 0,6 & & 0,667 & 0,672 & 0,624 \\
\hline MAE (previ & 6,551 & 6,635 & 6,122 & 5,951 & 6,319 \\
\hline$\nu \backslash \alpha$ & 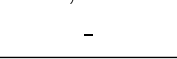 & 10 & 1,954 & 10,214 & 1,952 \\
\hline \multirow[b]{2}{*}{ Estatistica } & \multicolumn{5}{|c|}{ Seis outliers, $t=250,251,500,501,750,751$} \\
\hline & $\begin{array}{c}\text { GARCH } \\
\text { gaussiano }\end{array}$ & $\begin{array}{c}G A R C H \\
t \text {-Student }\end{array}$ & $\begin{array}{c}\text { GARCH } \\
\text { estável }\end{array}$ & $\begin{array}{c}G A S \\
t \text {-Student }\end{array}$ & $\begin{array}{c}\text { GAS } \\
\text { estável }\end{array}$ \\
\hline$\overline{\mathrm{AIC}}$ & 2899,308 & 2886,995 & 2877,151 & 2882,466 & 2871,779 \\
\hline$p$-valo1 (AD) & & 0,629 & 0,674 & 0,274 & 0,706 \\
\hline MAE (previsão) & 7,121 & 7,024 & 6,213 & 5,941 & 6,291 \\
\hline$\nu \backslash \alpha$ & 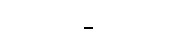 & 10 & 1,931 & 7,055 & 1,917 \\
\hline
\end{tabular}

Tabela 6.4: Medianas das estatisticas de ajustes para séries simuladas por processos $G A R C H(1,1)$ gaussianos com $\alpha_{0}=0,1, \alpha_{1}=0,1, \beta_{1}=0,8$ e outliers de tamanho $\rho=5$ 


\begin{tabular}{|c|c|c|c|c|c|}
\hline \multirow[b]{2}{*}{ Estatistica } & \multicolumn{5}{|c|}{ Um outlier, $t=500$} \\
\hline & $\begin{array}{c}\text { GARCH } \\
\text { gaussiano }\end{array}$ & $\begin{array}{l}\text { GARCH } \\
\text { t-Student }\end{array}$ & $\begin{array}{c}\text { GARCH } \\
\text { estável }\end{array}$ & $\begin{array}{c}G A S \\
t-S t u d e n t\end{array}$ & $\begin{array}{c}\text { GAS } \\
\text { estável }\end{array}$ \\
\hline $\mathrm{AIC}$ & 2902,075 & 2842,734 & 2821,947 & 2835,796 & 2815,653 \\
\hline$p$-valor & 0,261 & 0,43 & 0,586 & 0,636 & 0,563 \\
\hline MAE (pre & 7,195 & 6,714 & 6,095 & 6,159 & 6,195 \\
\hline & - & 9,92 & 1,98 & 10,128 & 1,982 \\
\hline \multirow[b]{2}{*}{ Estatística } & \multicolumn{5}{|c|}{ Dois outliers, $t=500,501$} \\
\hline & $\begin{array}{c}\text { GARCH } \\
\text { gaussiano }\end{array}$ & $\begin{array}{c}\text { GARCH } \\
\text { t-Student }\end{array}$ & $\begin{array}{c}\text { GARCH } \\
\text { estável }\end{array}$ & $\begin{array}{c}G A S \\
t-S t u d e n t\end{array}$ & $\begin{array}{l}\text { GAS } \\
\text { estável }\end{array}$ \\
\hline$\overline{\overline{\mathrm{AIC}}}$ & 2919,839 & 2862,142 & 2853,978 & 2864,425 & 2846,319 \\
\hline- & 0,205 & 372 & 0,593 & 0,378 & 0,545 \\
\hline MA & 7,605 & 6,798 & 5,591 & 5,72 & 5,618 \\
\hline & 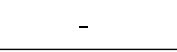 & 10 & 1,963 & 7,529 & 1,963 \\
\hline \multirow[b]{2}{*}{ Estatistica } & \multicolumn{5}{|c|}{ Dois outliers, $t=333,666$} \\
\hline & $\begin{array}{c}\text { GARCH } \\
\text { gaussiano }\end{array}$ & $\begin{array}{c}\text { GARCH } \\
t \text {-Student }\end{array}$ & $\begin{array}{c}\text { GARCH } \\
\text { estável }\end{array}$ & $\begin{array}{c}G A S \\
t \text {-Student }\end{array}$ & $\begin{array}{c}\text { GAS } \\
\text { estável }\end{array}$ \\
\hline & 2979,098 & 2854,132 & 2826,238 & 2843,343 & 2816,25 \\
\hline$p$-valor & 0 & 0,395 & 0,594 & 0,563 & 0,504 \\
\hline MAE (pre & 9,052 & 6,822 & 5,903 & 6,737 & 5,937 \\
\hline & - & 8,147 & 1,964 & 7,549 & 1,966 \\
\hline \multirow[b]{2}{*}{ Estatistica } & \multicolumn{5}{|c|}{ 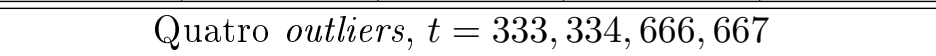 } \\
\hline & $\begin{array}{c}\text { GARCH } \\
\text { gaussiano }\end{array}$ & $\begin{array}{c}\text { GARCH } \\
t \text {-Student }\end{array}$ & $\begin{array}{c}\text { GARCH } \\
\text { estável }\end{array}$ & $\begin{array}{c}G A S \\
t \text {-Student }\end{array}$ & $\begin{array}{c}\text { GAS } \\
\text { estável }\end{array}$ \\
\hline & 3040,966 & 848 & 2898,482 & 2920,342 & 2895,5 \\
\hline 1 & 0 & 93 & 0,785 & 0,206 & 0,721 \\
\hline MAE (pre & 9,51 & 7,221 & 5,967 & 6,12 & 5,925 \\
\hline & - & 8,083 & 1,94 & 5,784 & 1,938 \\
\hline \multirow[b]{2}{*}{ Estatística } & \multicolumn{5}{|c|}{ Três outliers, $t=250,500,750$} \\
\hline & $\begin{array}{c}\text { GARCH } \\
\text { gaussiano }\end{array}$ & $\begin{array}{c}\text { GARCH } \\
t \text {-Student }\end{array}$ & $\begin{array}{c}\text { GARCH } \\
\text { estável }\end{array}$ & $\begin{array}{c}G A S \\
t \text {-Studen }\end{array}$ & $\begin{array}{c}\text { GAS } \\
\text { estável }\end{array}$ \\
\hline AICc & 3106,117 & 2930,932 & 2888,558 & 2912,713 & 2877,143 \\
\hline lor & 0 & 0,395 & 0,733 & 0,464 & 0,7 \\
\hline MAE (pre) & 10,449 & 29 & 6,188 & 7,276 & 6,221 \\
\hline (1) & 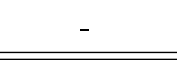 & 6,712 & 1,951 & 6,322 & 1,951 \\
\hline \multirow[b]{2}{*}{ Estatistic } & \multicolumn{5}{|c|}{ Seis outliers, $t=250,251,500,501,750,751$} \\
\hline & $\begin{array}{c}\text { GARCH } \\
\text { gaussiano }\end{array}$ & $\begin{array}{c}\text { GARCH } \\
t \text {-Student }\end{array}$ & $\begin{array}{c}\text { GARCH } \\
\text { estável }\end{array}$ & $\begin{array}{c}G A S \\
t \text {-Student }\end{array}$ & $\begin{array}{c}\text { GAS } \\
\text { estável }\end{array}$ \\
\hline & 3106,117 & 2930,932 & 2888,558 & 2912,713 & 2877,143 \\
\hline alor $(\mathrm{A}$ & 0 & 0,316 & 0,553 & 0,149 & 0,518 \\
\hline MAE (previsão) & 11,001 & 7,456 & 6,068 & 6,171 & 6,025 \\
\hline$\nu \backslash \alpha$ & - & 6,615 & 1,913 & 4,705 & 1,911 \\
\hline
\end{tabular}

Tabela 6.5: Medianas das estatísticas de ajustes para séries simuladas por processos GARCH(1,1) gaussianos com $\alpha_{0}=0,1, \alpha_{1}=0,1, \beta_{1}=0,8$ e outliers de tamanho $\rho=10$ 


\begin{tabular}{|c|c|c|c|c|c|}
\hline \multirow[b]{2}{*}{ Estatistica } & \multicolumn{5}{|c|}{ Um outlier, $t=500$} \\
\hline & $\begin{array}{c}\text { GARCH } \\
\text { gaussiano }\end{array}$ & $\begin{array}{c}\text { GARCH } \\
t \text {-Student }\end{array}$ & $\begin{array}{c}\text { GARCH } \\
\text { estável }\end{array}$ & $\begin{array}{c}G A S \\
t \text {-Student }\end{array}$ & $\begin{array}{c}\text { GAS } \\
\text { estável }\end{array}$ \\
\hline AIC & $\overline{04,411}$ & 2854,461 & 2824,352 & 2841,34 & 2816,996 \\
\hline alor & 0,007 & 0,516 & 0,685 & 0,635 & 0,69 \\
\hline MAE (pre & 9,213 & 6,233 & 5,823 & 6,028 & 5,965 \\
\hline t & - & 9,219 & 1,978 & 8,499 & 1,979 \\
\hline \multirow[b]{2}{*}{ Estatistica } & \multicolumn{5}{|c|}{ Dois outliers, $t=500,501$} \\
\hline & $\begin{array}{c}\text { GARCH } \\
\text { gaussiano }\end{array}$ & $\begin{array}{c}\text { GARCH } \\
t \text {-Student }\end{array}$ & $\begin{array}{c}\text { GARCH } \\
\text { estável }\end{array}$ & $\begin{array}{c}G A S \\
t \text {-Student }\end{array}$ & $\begin{array}{c}\text { GAS } \\
\text { estável }\end{array}$ \\
\hline AIC & 3024,138 & 2872,762 & 2852,493 & 2877,639 & 2843,486 \\
\hline$p$-valor & 0 & 0,481 & 0,667 & 0,488 & 0,605 \\
\hline MAE (prev & 9,802 & 6,405 & 5,362 & 6,007 & 5,375 \\
\hline$\nu \backslash \alpha$ & - & 9,239 & 1,962 & 6,438 & 1,963 \\
\hline \multirow[b]{2}{*}{ Estatística } & \multicolumn{5}{|c|}{$\overline{\text { Dois outliers, } t=333,666}$} \\
\hline & $\begin{array}{c}\text { GARCH } \\
\text { gaussiano }\end{array}$ & $\begin{array}{c}\text { GARCH } \\
t \text {-Student }\end{array}$ & $\begin{array}{c}\text { GARCH } \\
\text { estável }\end{array}$ & $\begin{array}{c}G A S \\
t \text {-Student }\end{array}$ & $\begin{array}{c}\text { GAS } \\
\text { estável }\end{array}$ \\
\hline & 3199,005 & 290 & 2861,48 & 289 & 853,369 \\
\hline$p$-valor & 0 & 0,329 & 0,67 & 0,359 & 0,599 \\
\hline MAE (prev & 12,87 & 6,877 & 5,796 & 6,981 & 6,034 \\
\hline$\nu \backslash \alpha$ & , & 6,952 & 1,964 & 6,578 & 1,966 \\
\hline \multirow[b]{2}{*}{ Estatistica } & \multicolumn{5}{|c|}{ Quatro outliers, $t=333,334,666,667$} \\
\hline & $\begin{array}{c}\text { GARCH } \\
\text { gaussiano }\end{array}$ & $\begin{array}{c}\text { GARCH } \\
\text { t-Student }\end{array}$ & $\begin{array}{c}G A R C H \\
\text { estável }\end{array}$ & $\begin{array}{c}G A S \\
t \text {-Student }\end{array}$ & $\begin{array}{c}\text { GAS } \\
\text { estável }\end{array}$ \\
\hline$\overline{\mathrm{A}}$ & 10,164 & 2923,787 & 2904,7 & 2934,127 & 2894,718 \\
\hline lor $(A$ & 0 & 0,388 & 0,737 & 0,279 & 0,628 \\
\hline (pres & 17,01 & 7,767 & 5,44 & 6,968 & 5,847 \\
\hline$\nu \backslash \alpha$ & 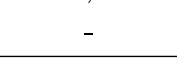 & 6,733 & 1,932 & 4,853 & 1,936 \\
\hline \multirow[b]{2}{*}{ Estatistica } & \multicolumn{5}{|c|}{ Três outliers, $t=250,500,750$} \\
\hline & $\begin{array}{c}\text { GARCH } \\
\text { gaussiano }\end{array}$ & $\begin{array}{c}\text { GARCH } \\
t \text {-Student }\end{array}$ & $\begin{array}{c}\text { GARCH } \\
\text { estável }\end{array}$ & $\begin{array}{c}G A S \\
t \text {-Student }\end{array}$ & $\begin{array}{c}\text { GAS } \\
\text { estável }\end{array}$ \\
\hline$\overline{\mathrm{AICc}}$ & 333,55 & 2896,625 & 2850,105 & 2877,832 & 2838,865 \\
\hline alor ( & 0 & & 0,554 & 0,23 & 0,523 \\
\hline MAE (prev & 17,366 & 6,762 & 5,141 & 6,412 & 5,135 \\
\hline$\nu \backslash \alpha$ & 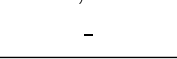 & 5,684 & 1,954 & 5,536 & 1,956 \\
\hline \multirow[b]{2}{*}{ Estatistica } & \multicolumn{5}{|c|}{ Seis outliers, $t=250,251,500,501,750,751$} \\
\hline & $\begin{array}{c}\text { GARCH } \\
\text { gaussiano }\end{array}$ & $\begin{array}{c}G A R C H \\
t \text {-Student }\end{array}$ & $\begin{array}{c}\text { GARCH } \\
\text { estável }\end{array}$ & $\begin{array}{c}G A S \\
t \text {-Student }\end{array}$ & $\begin{array}{c}\text { GAS } \\
\text { estável }\end{array}$ \\
\hline & 3404,021 & 2982,182 & 2956,167 & 2991,9 & 2945,523 \\
\hline$p$-valor (AD) & 0 & & 0,687 & 0,23 & 0,622 \\
\hline MAE (previsão) & 19,507 & 8,871 & 6,058 & 7,35 & 5,918 \\
\hline$\nu \backslash \alpha$ & - & 5,533 & 1,908 & 4,021 & 1,904 \\
\hline
\end{tabular}

Tabela 6.6: Medianas das estatisticas de ajustes para séries simuladas por processos GARCH(1,1) gaussianos com $\alpha_{0}=0,1, \alpha_{1}=0,1, \beta_{1}=0,8$ e outliers de tamanho $\rho=15$ 


\begin{tabular}{|c|c|c|c|c|c|}
\hline \multirow[b]{2}{*}{ Estatistica } & \multicolumn{5}{|c|}{ Um outlier, $t=500$} \\
\hline & $\begin{array}{c}\text { GARCH } \\
\text { gaussiano }\end{array}$ & $\begin{array}{c}\text { GARCH } \\
\text { t-Student }\end{array}$ & $\begin{array}{c}\text { GARCH } \\
\text { estável }\end{array}$ & $\begin{array}{c}G A S \\
t-S t u d e n t\end{array}$ & $\begin{array}{c}\text { GAS } \\
\text { estável }\end{array}$ \\
\hline HIV & $\overline{52,72}$ & 2856,999 & 2846,502 & 2852,008 & 2845,066 \\
\hline$p$-valor & 0,854 & 0,423 & 0,62 & 0,85 & 0,626 \\
\hline MAE (pre & 5,94 & 6,246 & 5,648 & 5,327 & 5,868 \\
\hline$\nu \backslash \alpha$ & - & 10 & 1,981 & 15,647 & 1,982 \\
\hline \multirow[b]{2}{*}{ Estatistica } & \multicolumn{5}{|c|}{ Dois outliers, $t=500,501$} \\
\hline & $\begin{array}{c}\text { GARCH } \\
\text { gaussiano }\end{array}$ & $\begin{array}{c}\text { GARCH } \\
\text { t-Student }\end{array}$ & $\begin{array}{c}G A R C H \\
\text { estável }\end{array}$ & $\begin{array}{c}G A S \\
t-S t u d e n t\end{array}$ & $\begin{array}{c}\text { GAS } \\
\text { estável }\end{array}$ \\
\hline & 2824,79 & 2830,067 & 2824,556 & 2817,871 & 2823,806 \\
\hline$p$-valor & 0,892 & 0,423 & 0,623 & 0,568 & 0,636 \\
\hline MAE (pr & 5,607 & 5,937 & 5,441 & 5,242 & 5,545 \\
\hline$\nu \backslash a$ & - & 10 & 1,981 & 12,637 & 1,972 \\
\hline \multirow[b]{2}{*}{ Estatistica } & \multicolumn{5}{|c|}{ Dois outliers, $t=333,666$} \\
\hline & $\begin{array}{c}\text { GARCH } \\
\text { gaussiano }\end{array}$ & $\begin{array}{c}\text { GARCH } \\
t \text {-Student }\end{array}$ & $\begin{array}{c}\text { GARCH } \\
\text { estável }\end{array}$ & $\begin{array}{c}G A S \\
t \text {-Student }\end{array}$ & $\begin{array}{c}\text { GAS } \\
\text { estável }\end{array}$ \\
\hline & 2838,383 & 2838,192 & 2831,654 & 2830,551 & 2827,018 \\
\hline$p$-valor & 0,823 & 0,448 & 0,649 & 0,817 & 0,653 \\
\hline MAE (pre & 6,201 & 6,473 & 5,952 & 5,984 & 5,903 \\
\hline & - & 10 & 1,966 & 12,608 & 1,967 \\
\hline \multirow[b]{2}{*}{ Estatística } & \multicolumn{5}{|c|}{ 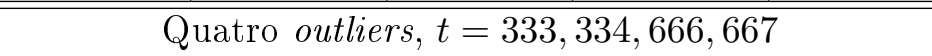 } \\
\hline & $\begin{array}{c}\text { GARCH } \\
\text { gaussiano }\end{array}$ & $\begin{array}{c}\text { GARCH } \\
t \text {-Student }\end{array}$ & $\begin{array}{c}\text { GARCH } \\
\text { estável }\end{array}$ & $\begin{array}{c}G A S \\
t \text {-Student }\end{array}$ & $\begin{array}{c}\text { GAS } \\
\text { estável }\end{array}$ \\
\hline & 2871,951 & 2862,052 & 2860,307 & 2856,708 & 2862,519 \\
\hline & 0 & 8 & 0,469 & 0,403 & 0,458 \\
\hline MAE (pr & 6,874 & 6,999 & 5,882 & 5,54 & 5,976 \\
\hline & 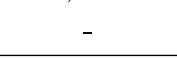 & 10 & 1,964 & 9,171 & 1,949 \\
\hline \multirow[b]{2}{*}{ Estatística } & \multicolumn{5}{|c|}{ Três outliers, $t=250,500,750$} \\
\hline & $\begin{array}{c}\text { GARCH } \\
\text { gaussiano }\end{array}$ & $\begin{array}{c}\text { GARCH } \\
t \text {-Student }\end{array}$ & $\begin{array}{c}\text { GARCH } \\
\text { estável }\end{array}$ & $\begin{array}{c}G A S \\
t \text {-Student }\end{array}$ & $\begin{array}{c}\text { GAS } \\
\text { estável }\end{array}$ \\
\hline AICc & 2886,202 & 2872,974 & 2864,613 & 2869,315 & 2858,577 \\
\hline alor & 0,639 & 0,515 & 0,704 & 0,751 & 0,697 \\
\hline MAE (pre & 6,209 & 6,21 & 5,561 & 5,606 & 5,685 \\
\hline$\nu \backslash \alpha$ & 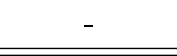 & 10 & 1,956 & 10,188 & 1,957 \\
\hline \multirow[b]{2}{*}{ Estatística } & \multicolumn{5}{|c|}{ Seis outliers, $t=250,251,500,501,750,751$} \\
\hline & $\begin{array}{c}\text { GARCH } \\
\text { gaussiano }\end{array}$ & $\begin{array}{c}\text { GARCH } \\
t \text {-Student }\end{array}$ & $\begin{array}{c}\text { GARCH } \\
\text { estável }\end{array}$ & $\begin{array}{c}G A S \\
t \text {-Student }\end{array}$ & $\begin{array}{c}\text { GAS } \\
\text { estável }\end{array}$ \\
\hline$\overline{\mathrm{AICc}}$ & 2910,557 & 2891,037 & 2890,708 & 2890,296 & 2896,943 \\
\hline alor & 0,616 & 0,584 & 0,756 & 0,285 & 0,712 \\
\hline MAE (previsão) & 6,865 & 6,787 & 5,622 & 5,339 & 5,673 \\
\hline$\nu \backslash \alpha$ & - & 10 & 1,945 & 7,469 & 1,926 \\
\hline
\end{tabular}

Tabela 6.7: Medianas das estatísticas de ajustes para séries simuladas por processos GARCH(1,1) gaussianos com $\alpha_{0}=0,3, \alpha_{1}=0,15, \beta_{1}=0,55$ e outliers de tamanho $\rho=5$ 


\begin{tabular}{|c|c|c|c|c|c|}
\hline \multirow[b]{2}{*}{ Estatistica } & \multicolumn{5}{|c|}{ Um outlier, $t=500$} \\
\hline & $\begin{array}{c}\text { GARCH } \\
\text { gaussiano }\end{array}$ & $\begin{array}{c}\text { GARCH } \\
t \text {-Student }\end{array}$ & $\begin{array}{c}\text { GARCH } \\
\text { estável }\end{array}$ & $\begin{array}{c}G A S \\
t \text {-Student }\end{array}$ & $\begin{array}{c}\text { GAS } \\
\text { estável }\end{array}$ \\
\hline HIC & 20,003 & 2865,998 & 2843,506 & 2861,04 & 2835,648 \\
\hline alor & 0,188 & 0,428 & 0,648 & 0,621 & 0,619 \\
\hline E (pre & 6,839 & 6,311 & 5,883 & 5,747 & 6,037 \\
\hline ( & - & 10 & 1,981 & 10,376 & 1,983 \\
\hline \multirow[b]{2}{*}{ Estatistica } & \multicolumn{5}{|c|}{ Dois outliers, $t=500,501$} \\
\hline & $\begin{array}{c}\text { GARCH } \\
\text { gaussiano }\end{array}$ & $\begin{array}{c}\text { GARCH } \\
t \text {-Student }\end{array}$ & $\begin{array}{c}\text { GARCH } \\
\text { estável }\end{array}$ & $\begin{array}{c}G A S \\
t-S t u d e n t\end{array}$ & $\begin{array}{c}\text { GAS } \\
\text { estável }\end{array}$ \\
\hline 710 & 2928,857 & 2867,591 & 2857,98 & 2866,177 & 2855,349 \\
\hline$p$-valor & 0,157 & 0,438 & 0,666 & 0,357 & 0,626 \\
\hline MAE (prev & 7,176 & 6,557 & 6,129 & 6,039 & 6,064 \\
\hline$\nu \backslash \alpha$ & 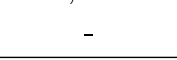 & 10 & 1,971 & 7,566 & 1,967 \\
\hline \multirow[b]{2}{*}{ Estatistica } & \multicolumn{5}{|c|}{$\overline{\text { Dois outliers, } t=333,666}$} \\
\hline & $\begin{array}{c}\text { GARCH } \\
\text { gaussiano }\end{array}$ & $\begin{array}{c}\text { GARCH } \\
t \text {-Student }\end{array}$ & $\begin{array}{c}\text { GARCH } \\
\text { estável }\end{array}$ & $\begin{array}{c}G A S \\
t \text {-Student }\end{array}$ & $\begin{array}{c}\text { GAS } \\
\text { estável }\end{array}$ \\
\hline & 2995,354 & 289 & 2863,778 & 2888,61 & 2857,04 \\
\hline alor & 0 & 0 , & 0,595 & 0,475 & 0,578 \\
\hline MAE (prev & 8,264 & 6,563 & 5,615 & 5,889 & 5,703 \\
\hline$\nu \backslash \alpha$ & - & 8,446 & 1,964 & 7,891 & 1,965 \\
\hline \multirow[b]{2}{*}{ Estatistica } & \multicolumn{5}{|c|}{ Quatro outliers, $t=333,334,666,667$} \\
\hline & $\begin{array}{c}\text { GARCH } \\
\text { gaussiano }\end{array}$ & $\begin{array}{c}\text { GARCH } \\
\text { t-Student }\end{array}$ & $\begin{array}{c}G A R C H \\
\text { estável }\end{array}$ & $\begin{array}{c}G A S \\
t \text {-Student }\end{array}$ & $\begin{array}{c}\text { GAS } \\
\text { estável }\end{array}$ \\
\hline AICc & 13,665 & 893,973 & 2880,649 & 2900,54 & 2882,615 \\
\hline lor $(A$ & 0 & & 0,663 & 0,15 & 0,612 \\
\hline (pres & 8,561 & 1 & 5,868 & 5,512 & 5,83 \\
\hline$\nu \backslash \alpha$ & 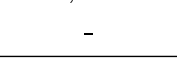 & 8,121 & 1,946 & 5,708 & 1,934 \\
\hline \multirow[b]{2}{*}{ Estatistica } & \multicolumn{5}{|c|}{ Três outliers, $t=250,500,750$} \\
\hline & $\begin{array}{c}\text { GARCH } \\
\text { gaussiano }\end{array}$ & $\begin{array}{c}\text { GARCH } \\
t \text {-Student }\end{array}$ & $\begin{array}{c}\text { GARCH } \\
\text { estável }\end{array}$ & $\begin{array}{c}G A S \\
t \text {-Student }\end{array}$ & $\begin{array}{c}\text { GAS } \\
\text { estável }\end{array}$ \\
\hline$\overline{\mathrm{AICc}}$ & 3092,841 & 2926,221 & 2889,426 & 2917,216 & 2881,553 \\
\hline alor ( & 0 & & 0,61 & 0,409 & 0,567 \\
\hline MAE (prev & 9,648 & 6,911 & 6,125 & 6,465 & 6,11 \\
\hline$\nu \backslash \alpha$ & 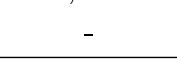 & 6,892 & 1,949 & 6,433 & 1,949 \\
\hline \multirow[b]{2}{*}{ Estatistica } & \multicolumn{5}{|c|}{ Seis outliers, $t=250,251,500,501,750,751$} \\
\hline & $\begin{array}{c}\text { GARCH } \\
\text { gaussiano }\end{array}$ & $\begin{array}{c}\text { GARCH } \\
t \text {-Student }\end{array}$ & $\begin{array}{c}\text { GARCH } \\
\text { estável }\end{array}$ & $\begin{array}{c}G A S \\
t \text {-Student }\end{array}$ & $\begin{array}{c}\text { GAS } \\
\text { estável }\end{array}$ \\
\hline$\overline{\mathrm{AIC}}$ & 3108,17 & 2920,872 & 2904,361 & 2930,681 & 2906,089 \\
\hline$p$-valor (AD) & 0 & & 0,724 & 0,071 & 0,685 \\
\hline MAE (previsão) & 11,049 & 7,493 & 5,826 & 5,458 & 5,729 \\
\hline$\nu \backslash \alpha$ & - & 6,721 & 1,923 & 4,819 & 1,911 \\
\hline
\end{tabular}

Tabela 6.8: Medianas das estatísticas de ajustes para séries simuladas por processos $G A R C H(1,1)$ gaussianos com $\alpha_{0}=0,3, \alpha_{1}=0,15, \beta_{1}=0,55$ e outliers de tamanho $\rho=10$ 


\begin{tabular}{|c|c|c|c|c|c|}
\hline \multirow[b]{2}{*}{ Estatistica } & \multicolumn{5}{|c|}{ Um outlier, $t=500$} \\
\hline & $\begin{array}{c}\text { GARCH } \\
\text { gaussiano }\end{array}$ & $\begin{array}{c}\text { GARCH } \\
\text { t-Student }\end{array}$ & $\begin{array}{c}\text { GARCH } \\
\text { estável }\end{array}$ & $\begin{array}{c}G A S \\
t-S t u d e n t\end{array}$ & $\begin{array}{c}\text { GAS } \\
\text { estável }\end{array}$ \\
\hline & 13,593 & 2860,895 & 2829,877 & 2851,391 & 2826,49 \\
\hline$p$-valor $(\mathrm{A}$ & 0 & 0,412 & 0,616 & 0,591 & 0,626 \\
\hline MAE (pres & 9,341 & 6,021 & 5,316 & 5,193 & 5,402 \\
\hline$\nu \backslash \alpha$ & - & 9,204 & 1,981 & 8,464 & 1,981 \\
\hline \multirow[b]{2}{*}{ Estatistica } & \multicolumn{5}{|c|}{ Dois outliers, $t=500,501$} \\
\hline & $\begin{array}{c}\text { GARCH } \\
\text { gaussiano }\end{array}$ & $\begin{array}{c}\text { GARCH } \\
\text { t-Student }\end{array}$ & $\begin{array}{c}G A R C H \\
\text { estável }\end{array}$ & $\begin{array}{c}G A S \\
t-S t u d e n t\end{array}$ & $\begin{array}{c}\text { GAS } \\
\text { estável }\end{array}$ \\
\hline & 3025,561 & 2871,892 & 2860,638 & 2871,604 & 2856,732 \\
\hline$p$-valor & 0,002 & 0,427 & 0,661 & 0,28 & 0,627 \\
\hline MAE (pr & 10,597 & 6,777 & 5,205 & 5,168 & 5,299 \\
\hline$\nu \backslash \alpha$ & - & 9,215 & 1,965 & 6,37 & 1,963 \\
\hline \multirow[b]{2}{*}{ Estatística } & \multicolumn{5}{|c|}{$\overline{\text { Dois } \text { outliers, } t=333,666}$} \\
\hline & $\begin{array}{c}\text { GARCH } \\
\text { gaussiano }\end{array}$ & $\begin{array}{c}\text { GARCH } \\
\text { t-Student }\end{array}$ & $\begin{array}{c}\text { GARCH } \\
\text { estável }\end{array}$ & $\begin{array}{c}G A S \\
t \text {-Student }\end{array}$ & $\begin{array}{c}\text { GAS } \\
\text { estável }\end{array}$ \\
\hline & 3182,925 & 2884,066 & 2847,783 & 2880,442 & 2840,949 \\
\hline$p$-valor & 0 & 0,319 & 0,662 & 0,436 & 0,63 \\
\hline MAE (pre & 12,877 & 6,308 & 5,016 & 5,929 & 4,945 \\
\hline & - & 7,016 & 1,961 & 6,6 & 1,963 \\
\hline \multirow[b]{2}{*}{ Estatística } & \multicolumn{5}{|c|}{ Quatro outliers, $t=333,334,666,667$} \\
\hline & $\begin{array}{c}\text { GARCH } \\
\text { gaussiano }\end{array}$ & $\begin{array}{c}\text { GARCH } \\
\text { t-Student }\end{array}$ & $\begin{array}{c}\text { GARCH } \\
\text { estável }\end{array}$ & $\begin{array}{c}G A S \\
t \text {-Student }\end{array}$ & $\begin{array}{c}\text { GAS } \\
\text { estável }\end{array}$ \\
\hline & 3211,842 & 2921,551 & 2895,937 & 2932,342 & 2896,399 \\
\hline . & 0 & 93 & 0,626 & 0,099 & 0,571 \\
\hline MAE (pr & 15,624 & 7,035 & 5,213 & 5,401 & 5,263 \\
\hline$\nu \backslash$ & 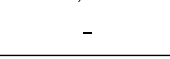 & 7,061 & 1,946 & 5,037 & 1,939 \\
\hline \multirow[b]{2}{*}{ Estatística } & \multicolumn{5}{|c|}{ Três outliers, $t=250,500,750$} \\
\hline & $\begin{array}{c}\text { GARCH } \\
\text { gaussiano }\end{array}$ & $\begin{array}{c}\text { GARCH } \\
\text { t-Student }\end{array}$ & $\begin{array}{c}\text { GARCH } \\
\text { estável }\end{array}$ & $\begin{array}{c}G A S \\
t \text {-Student }\end{array}$ & $\begin{array}{c}\text { GAS } \\
\text { estável }\end{array}$ \\
\hline $\mathrm{AIC}$ & 3342,663 & 2935,007 & 2884,6 & 2926,029 & 2874,057 \\
\hline alor & 0 & 0,267 & 0,636 & 0,315 & 0,633 \\
\hline MAE (pre & 16,961 & 7,553 & 5,598 & 6,589 & 5,721 \\
\hline$\nu \backslash \alpha$ & 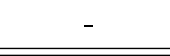 & 5,869 & 1,951 & 5,699 & 1,951 \\
\hline \multirow[b]{2}{*}{ Estatística } & \multicolumn{5}{|c|}{ Seis outliers, $t=250,251,500,501,750,751$} \\
\hline & $\begin{array}{c}\text { GARCH } \\
\text { gaussiano }\end{array}$ & $\begin{array}{c}\text { GARCH } \\
t \text {-Student }\end{array}$ & $\begin{array}{c}\text { GARCH } \\
\text { estável }\end{array}$ & $\begin{array}{c}G A S \\
t \text {-Student }\end{array}$ & $\begin{array}{c}\text { GAS } \\
\text { estável }\end{array}$ \\
\hline$\overline{\mathrm{AICc}}$ & 3378,533 & 2953,805 & 2931,003 & 2973,378 & 2927,376 \\
\hline ralor & 0 & 0,222 & 0,528 & 0,153 & 0,449 \\
\hline MAE (previsão) & 19,922 & 8,466 & 5,881 & 6,501 & 5,683 \\
\hline$\nu \backslash \alpha$ & - & 5,838 & 1,919 & 4,025 & 1,906 \\
\hline
\end{tabular}

Tabela 6.9: Medianas das estatísticas de ajustes para séries simuladas por processos GARCH(1,1) gaussianos com $\alpha_{0}=0,3, \alpha_{1}=0,15, \beta_{1}=0,55$ e outliers de tamanho $\rho=15$ 


\begin{tabular}{|c|c|c|c|c|c|}
\hline \multirow[b]{2}{*}{ Estatistica } & \multicolumn{5}{|c|}{ Um outlier, $t=500$} \\
\hline & $\begin{array}{c}\text { GARCH } \\
\text { gaussiano }\end{array}$ & $\begin{array}{c}\text { GARCH } \\
t \text {-Student }\end{array}$ & $\begin{array}{c}\text { GARCH } \\
\text { estável }\end{array}$ & $\begin{array}{c}G A S \\
t \text {-Student }\end{array}$ & $\begin{array}{l}\text { GAS } \\
\text { estável }\end{array}$ \\
\hline AIC & 27,331 & 2825,88 & 2819,666 & 2821,754 & 2813,608 \\
\hline alor & 0,85 & 0,367 & 0,638 & 0,789 & 0,619 \\
\hline MAE (previsão & 5,764 & 5,929 & 5,686 & 5,425 & 5,817 \\
\hline ( & - & 10 & 1,98 & 15,766 & 1,982 \\
\hline \multirow[b]{2}{*}{ Estatistica } & \multicolumn{5}{|c|}{ Dois outliers, $t=500,501$} \\
\hline & $\begin{array}{c}\text { GARCH } \\
\text { gaussiano }\end{array}$ & $\begin{array}{c}\text { GARCH } \\
t \text {-Student }\end{array}$ & $\begin{array}{c}\text { GARCH } \\
\text { estável }\end{array}$ & $\begin{array}{c}G A S \\
t-S t u d e n t\end{array}$ & $\begin{array}{c}\text { GAS } \\
\text { estável }\end{array}$ \\
\hline $\mathrm{AICc}$ & 2855,618 & 2858,172 & 2856,645 & 2853,736 & 2853,616 \\
\hline$p$-valor & 0,879 & 0,385 & 0,701 & 0,573 & 0,631 \\
\hline MAE (prev & 5,868 & 6,358 & 5,282 & 5,109 & 5,574 \\
\hline$\nu \backslash \alpha$ & - & 10 & 1,984 & 12,44 & 1,977 \\
\hline \multirow[b]{2}{*}{ Estatistica } & \multicolumn{5}{|c|}{ 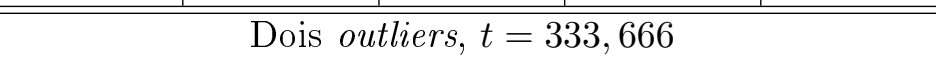 } \\
\hline & $\begin{array}{c}\text { GARCH } \\
\text { gaussiano }\end{array}$ & $\begin{array}{c}\text { GARCH } \\
t \text {-Student }\end{array}$ & $\begin{array}{c}\text { GARCH } \\
\text { estável }\end{array}$ & $\begin{array}{c}G A S \\
t \text {-Student }\end{array}$ & $\begin{array}{c}\text { GAS } \\
\text { estável }\end{array}$ \\
\hline & 2857,149 & - & 2844 & 2851,30 & 2839,8 \\
\hline$p$-valor & 0,829 & 0,434 & 0,689 & 0,729 & 0,662 \\
\hline MAE (prev & 5,977 & 6,132 & 5,256 & 5,144 & 5,373 \\
\hline$\nu \backslash \alpha$ & - & 10 & 1,967 & 11,868 & 1,968 \\
\hline \multirow[b]{2}{*}{ Estatistica } & \multicolumn{5}{|c|}{ Quatro outliers, $t=333,334,666,667$} \\
\hline & $\begin{array}{c}\text { GARCH } \\
\text { gaussiano }\end{array}$ & $\begin{array}{c}\text { GARCH } \\
\text { t-Student }\end{array}$ & $\begin{array}{c}\text { GARCH } \\
\text { estável }\end{array}$ & $\begin{array}{c}G A S \\
t \text {-Student }\end{array}$ & $\begin{array}{c}\text { GAS } \\
\text { estável }\end{array}$ \\
\hline$\overline{\mathrm{AICc}}$ & 2881,446 & 2872,947 & 2870,095 & 2867,889 & 2872,834 \\
\hline lor $(A$ & 0,753 & 0,437 & 0,631 & 0,423 & 0,657 \\
\hline (prev & 6,117 & 6,237 & 5,655 & 5,42 & 5,71 \\
\hline$\nu \backslash \alpha$ & - & 10 & 1,962 & 8,784 & 1,945 \\
\hline \multirow[b]{2}{*}{ Estatistica } & \multicolumn{5}{|c|}{ Três outliers, $t=250,500,750$} \\
\hline & $\begin{array}{c}\text { GARCH } \\
\text { gaussiano }\end{array}$ & $\begin{array}{c}\text { GARCH } \\
t \text {-Student }\end{array}$ & $\begin{array}{c}\text { GARCH } \\
\text { estável }\end{array}$ & $\begin{array}{c}G A S \\
t \text {-Student }\end{array}$ & $\begin{array}{c}\text { GAS } \\
\text { estável }\end{array}$ \\
\hline$\overline{\mathrm{AICc}}$ & 2874,537 & 2862,129 & 2853,573 & 2861,191 & 2846,409 \\
\hline alor ( & 0,62 & & 0,704 & 0,785 & 0,707 \\
\hline MAE (previ & 6,389 & 6,433 & 5,541 & 5,342 & 5,611 \\
\hline$\nu \backslash \alpha$ & 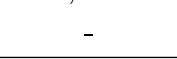 & 10 & 1,954 & 10,162 & 1,954 \\
\hline \multirow[b]{2}{*}{ Estatistica } & \multicolumn{5}{|c|}{ Seis outliers, $t=250,251,500,501,750,751$} \\
\hline & $\begin{array}{c}\text { GARCH } \\
\text { gaussiano }\end{array}$ & $\begin{array}{c}G A R C H \\
t \text {-Student }\end{array}$ & $\begin{array}{c}\text { GARCH } \\
\text { estável }\end{array}$ & $\begin{array}{c}G A S \\
t \text {-Student }\end{array}$ & $\begin{array}{c}\text { GAS } \\
\text { estável }\end{array}$ \\
\hline & 2892,98 & 2877,158 & 2870,448 & 2871,999 & 2878,403 \\
\hline$p$-valor (AD) & 0,617 & 0,451 & 0,737 & 0,284 & 0,726 \\
\hline MAE (previsão) & 6,754 & 6,804 & 5,553 & 5,3 & 5,604 \\
\hline$\nu \backslash \alpha$ & 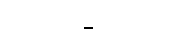 & 10 & 1,952 & 7,528 & 1,926 \\
\hline
\end{tabular}

Tabela 6.10: Medianas das estatísticas de ajustes para séries simuladas por processos GARCH(1,1) gaussianos com $\alpha_{0}=0,5, \alpha_{1}=0,2, \beta_{1}=0,3$ e outliers de tamanho $\rho=5$ 


\begin{tabular}{|c|c|c|c|c|c|}
\hline \multirow[b]{2}{*}{ Estatistica } & \multicolumn{5}{|c|}{ Um outlier, $t=500$} \\
\hline & $\begin{array}{c}\text { GARCH } \\
\text { gaussiano }\end{array}$ & $\begin{array}{c}\text { GARCH } \\
\text { t-Student }\end{array}$ & $\begin{array}{c}\text { GARCH } \\
\text { estável }\end{array}$ & $\begin{array}{c}G A S \\
t-S t u d e n t\end{array}$ & $\begin{array}{c}\text { GAS } \\
\text { estável }\end{array}$ \\
\hline & $\overline{05,224}$ & 2847,533 & 2832,213 & 2845,758 & 2825,968 \\
\hline alor & 0,253 & 0,347 & 0,521 & 0,677 & 0,495 \\
\hline MAE (pre & 6,396 & 5,835 & 5,188 & 5,083 & 5,301 \\
\hline$\nu \backslash \alpha$ & - & 10 & 1,979 & 9,757 & 1,98 \\
\hline \multirow[b]{2}{*}{ Estatistica } & \multicolumn{5}{|c|}{ Dois outliers, $t=500,501$} \\
\hline & $\begin{array}{c}\text { GARCH } \\
\text { gaussiano }\end{array}$ & $\begin{array}{c}\text { GARCH } \\
\text { t-Student }\end{array}$ & $\begin{array}{c}\text { GARCH } \\
\text { estável }\end{array}$ & $\begin{array}{c}G A S \\
t-S t u d e n t\end{array}$ & $\begin{array}{c}\text { GAS } \\
\text { estável }\end{array}$ \\
\hline$\overline{\mathrm{AIC}}$ & 2912,55 & 2849,048 & 2839,911 & 2849,922 & 2836,448 \\
\hline$p$-valor & 0,252 & 0,412 & 0,701 & 0,344 & 0,671 \\
\hline MAE (pre & 6,919 & 6,014 & 5,179 & 5,1 & 5,225 \\
\hline$\nu \backslash \alpha$ & - & 10 & 1,974 & 7,518 & 1,964 \\
\hline \multirow[b]{2}{*}{ Estatística } & \multicolumn{5}{|c|}{ Dois outliers, $t=333,666$} \\
\hline & $\begin{array}{c}\text { GARCH } \\
\text { gaussiano }\end{array}$ & $\begin{array}{c}\text { GARCH } \\
\text { t-Student }\end{array}$ & $\begin{array}{c}\text { GARCH } \\
\text { estável }\end{array}$ & $\begin{array}{c}G A S \\
t-S t u d e n t\end{array}$ & $\begin{array}{c}\text { GAS } \\
\text { estável }\end{array}$ \\
\hline & 2987,12 & 2874,923 & 2853,879 & 2872,126 & 2848,775 \\
\hline$p$-valor & 0 & 0,386 & 0,685 & 0,564 & 0,692 \\
\hline MAE (pre & 8,027 & 6,539 & 5,773 & 5,803 & 5,792 \\
\hline$\nu \backslash$ & - & 8,143 & 1,962 & 7,388 & 1,965 \\
\hline \multirow[b]{2}{*}{ Estatística } & \multicolumn{5}{|c|}{ Quatro outliers, $t=333,334,666,667$} \\
\hline & $\begin{array}{c}\text { GARCH } \\
\text { gaussiano }\end{array}$ & $\begin{array}{c}\text { GARCH } \\
\text { t-Student }\end{array}$ & $\begin{array}{c}\text { GARCH } \\
\text { estável }\end{array}$ & $\begin{array}{c}G A S \\
t-S t u d e n t\end{array}$ & $\begin{array}{c}G A S \\
\text { estável }\end{array}$ \\
\hline & 3012,132 & $\overline{4,1}$ & 2880,281 & 2897,69 & 2881,903 \\
\hline 10 & 0 & 27 & 0,587 & 0,143 & 0,546 \\
\hline MAE (pr & 9,396 & 6,808 & 5,247 & 5,177 & 5,212 \\
\hline$\nu \backslash$ & - & 8,265 & 1,955 & 5,686 & 1,941 \\
\hline \multirow[b]{2}{*}{ Estatistica } & \multicolumn{5}{|c|}{ Três outliers, $t=250,500,750$} \\
\hline & $\begin{array}{c}\text { GARCH } \\
\text { gaussiano }\end{array}$ & $\begin{array}{c}\text { GARCH } \\
\text { t-Student }\end{array}$ & $\begin{array}{c}\text { GARCH } \\
\text { estável }\end{array}$ & $\begin{array}{c}G A S \\
t-S t u d e n t\end{array}$ & $\begin{array}{c}G A S \\
\text { estável }\end{array}$ \\
\hline AICc & 3084,484 & 2904,818 & 2873,244 & 2893,865 & 2866,855 \\
\hline alor & 0 & & 0,626 & 0,398 & 0,628 \\
\hline MAE (pr & 9,785 & 93 & 5,332 & 5,627 & 5,425 \\
\hline$\nu \backslash \alpha$ & _ & 6,875 & 1,95 & 6,338 & 1,95 \\
\hline \multirow[b]{2}{*}{ Estatística } & \multicolumn{5}{|c|}{ Seis outliers, $t=250,251,500,501,750,751$} \\
\hline & $\begin{array}{c}\text { GARCH } \\
\text { gaussiano }\end{array}$ & $\begin{array}{c}\text { GARCH } \\
\text { t-Student }\end{array}$ & $\begin{array}{c}\text { GARCH } \\
\text { estável }\end{array}$ & $\begin{array}{c}G A S \\
t-S t u d e n t\end{array}$ & $\begin{array}{c}\text { GAS } \\
\text { estável }\end{array}$ \\
\hline & 3092,143 & 2919,461 & 2904,207 & 2928,489 & 2912,15 \\
\hline -valor $(\mathrm{A}$ & 0 & 0,299 & 0,644 & 0,067 & 0,588 \\
\hline MAE (previsão) & 10,754 & 7,224 & 5,466 & 5,502 & 5,493 \\
\hline$\nu \backslash \alpha$ & - & 6,818 & 1,939 & 4,739 & 1,91 \\
\hline
\end{tabular}

Tabela 6.11: Medianas das estatísticas de ajustes para séries simuladas por processos $G A R C H(1,1)$ gaussianos com $\alpha_{0}=0,5, \alpha_{1}=0,2, \beta_{1}=0,3$ e outliers de tamanho $\rho=10$ 


\begin{tabular}{|c|c|c|c|c|c|}
\hline \multirow[b]{2}{*}{ Estatistica } & \multicolumn{5}{|c|}{ Um outlier, $t=500$} \\
\hline & $\begin{array}{c}\text { GARCH } \\
\text { gaussiano }\end{array}$ & $\begin{array}{c}\text { GARCH } \\
t \text {-Student }\end{array}$ & $\begin{array}{c}\text { GARCH } \\
\text { estável }\end{array}$ & $\begin{array}{c}G A S \\
t \text {-Student }\end{array}$ & $\begin{array}{c}\text { GAS } \\
\text { estável }\end{array}$ \\
\hline AIC & 05,317 & 2863,396 & 2837,203 & 2859,974 & 2829,513 \\
\hline alor & 0,002 & 0,402 & 0,64 & 0,595 & 0,658 \\
\hline E (pre & 9,1 & 5,973 & 5,384 & 5,65 & 5,509 \\
\hline to & - & 9,663 & 1,98 & 8,213 & 1,982 \\
\hline \multirow[b]{2}{*}{ Estatistica } & \multicolumn{5}{|c|}{ Dois outliers, $t=500,501$} \\
\hline & $\begin{array}{c}\text { GARCH } \\
\text { gaussiano }\end{array}$ & $\begin{array}{c}\text { GARCH } \\
t \text {-Student }\end{array}$ & $\begin{array}{c}\text { GARCH } \\
\text { estável }\end{array}$ & $\begin{array}{c}G A S \\
t-S t u d e n t\end{array}$ & $\begin{array}{c}\text { GAS } \\
\text { estável }\end{array}$ \\
\hline$\overline{\mathrm{AIC}}$ & 3041,886 & 2877,061 & 2861,955 & 2882,342 & 2864,47 \\
\hline$p$-valor & 0,009 & 0,418 & 0,56 & 0,235 & 0,588 \\
\hline MAE (pre & 9,826 & 6,349 & 5,903 & 5,368 & 5,834 \\
\hline$\nu \backslash \alpha$ & - & 9,675 & 1,974 & 6,556 & 1,966 \\
\hline \multirow[b]{2}{*}{ Estatistica } & \multicolumn{5}{|c|}{ 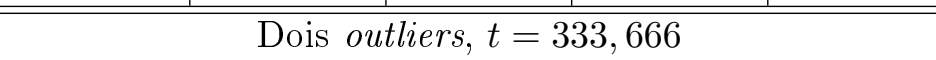 } \\
\hline & $\begin{array}{c}\text { GARCH } \\
\text { gaussiano }\end{array}$ & $\begin{array}{c}\text { GARCH } \\
t \text {-Student }\end{array}$ & $\begin{array}{c}\text { GARCH } \\
\text { estável }\end{array}$ & $\begin{array}{c}G A S \\
t \text {-Student }\end{array}$ & $\begin{array}{c}\text { GAS } \\
\text { estável }\end{array}$ \\
\hline & $\overline{92,5}$ & 2896 & 2861,308 & 2891,62 & 2849,89 \\
\hline$p$-valor & 0 & 0,256 & 0,643 & 0,349 & 0,602 \\
\hline MAE (prev & 14,074 & 7,169 & 5,803 & 6 & 5,802 \\
\hline$\nu \backslash \alpha$ & t & 7,193 & 1,965 & 6,637 & 1,966 \\
\hline \multirow[b]{2}{*}{ Estatistica } & \multicolumn{5}{|c|}{ Quatro outliers, $t=333,334,666,667$} \\
\hline & $\begin{array}{c}\text { GARCH } \\
\text { gaussiano }\end{array}$ & $\begin{array}{c}\text { GARCH } \\
\text { t-Student }\end{array}$ & $\begin{array}{c}G A R C H \\
\text { estável }\end{array}$ & $\begin{array}{c}G A S \\
t \text {-Student }\end{array}$ & $\begin{array}{c}\text { GAS } \\
\text { estável }\end{array}$ \\
\hline AICc & 90,696 & 2902,434 & 2883,052 & $2916,07:$ & 2883,555 \\
\hline lor $(A$ & 0 & 0,272 & 0,616 & 0,088 & 0,537 \\
\hline (pres & 16,608 & 5 & 5,657 & 5,503 & 5,688 \\
\hline$\nu \backslash \alpha$ & 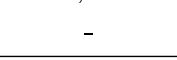 & 7,049 & 1,953 & 4,934 & 1,935 \\
\hline \multirow[b]{2}{*}{ Estatistica } & \multicolumn{5}{|c|}{ Três outliers, $t=250,500,750$} \\
\hline & $\begin{array}{c}\text { GARCH } \\
\text { gaussiano }\end{array}$ & $\begin{array}{c}\text { GARCH } \\
t \text {-Student }\end{array}$ & $\begin{array}{c}\text { GARCH } \\
\text { estável }\end{array}$ & $\begin{array}{c}G A S \\
t \text {-Student }\end{array}$ & $\begin{array}{c}\text { GAS } \\
\text { estável }\end{array}$ \\
\hline$\overline{\mathrm{AICc}}$ & 3350,426 & 2921,634 & 2878,448 & 2916,613 & 2871,264 \\
\hline alor ( & 0 & & 0,586 & 0,351 & 0,581 \\
\hline MAE (prev & 15,618 & 7,384 & 5,264 & 5,701 & 5,31 \\
\hline$\nu \backslash \alpha$ & - & 5,809 & 1,947 & 5,564 & 1,946 \\
\hline \multirow[b]{2}{*}{ Estatistica } & \multicolumn{5}{|c|}{ Seis outliers, $t=250,251,500,501,750,751$} \\
\hline & $\begin{array}{c}\text { GARCH } \\
\text { gaussiano }\end{array}$ & $\begin{array}{c}G A R C H \\
t \text {-Student }\end{array}$ & $\begin{array}{c}\text { GARCH } \\
\text { estável }\end{array}$ & $\begin{array}{c}G A S \\
t \text {-Student }\end{array}$ & $\begin{array}{c}\text { GAS } \\
\text { estável }\end{array}$ \\
\hline & 3358,428 & 2944,876 & 2917,826 & 2964,963 & 2926,353 \\
\hline$p$-valo1 (AD) & 0 & & 0,552 & 0,073 & 0,496 \\
\hline MAE (previsão) & 19,525 & 8,127 & 5,176 & 5,621 & 5,072 \\
\hline$\nu \backslash \alpha$ & - & 5,881 & 1,927 & 4,032 & 1,909 \\
\hline
\end{tabular}

Tabela 6.12: Medianas das estatísticas de ajustes para séries simuladas por processos GARCH(1,1) gaussianos com $\alpha_{0}=0,5, \alpha_{1}=0,2, \beta_{1}=0,3$ e outliers de tamanho $\rho=15$ 
Na grande maioria dos casos, o modelo GAS estável leva vantagem em termos de ajuste. Dos 54 cenários, o modelo apresenta menor valor para mediana dos AICs corrigidos em 41, seguido pelos modelos GARCH estável (8) e GAS $t$-Student (5). Observa-se que o modelo GAS estável leva maior vantagem nos cenários considerados para o conjunto de parâmetros $\alpha_{0}=0,1, \alpha_{1}=0,1$, $\beta_{1}=0,8$, nos quais tem melhor desempenho em 17 dos 18 possíveis. Essa vantagem cai no conjunto de parâmetros seguinte $\alpha_{0}=0,3, \alpha_{1}=0,15, \beta_{1}=0,55$, em que é melhor em 13 dos 18 cenários, e ainda mais no último conjunto de parâmetros $\alpha_{0}=0,5, \alpha_{1}=0,2, \beta_{1}=0,3$, em que é melhor em 11 dos 18 cenários.

Em termos de previsão da volatilidade, o modelo GAS estável não é tão eficiente quanto os modelos GARCH estável e GAS $t$-Student. Dos 54 cenários, o modelo proposto tem melhor desempenho em termos do MAE em apenas 10. O melhor modelo é o GAS $t$-Student, com vantagem em 25 cenários, seguido pelo GARCH estável, melhor em 19.

Em relação à qualidade de ajuste dos modelos, é possível observar um padrão. Quanto menor o número de outliers e menor os seus valores, a mediana dos p-valores do teste AD do modelo GAS $t$-Student é alta, maior que as correspondentes medianas dos modelos GARCH e GAS estáveis. No entanto, quando o número de outliers é maior, assim como seus valores, os modelos estáveis apresentam clara vantagem na qualidade de ajuste. Em todos os casos, observa-se que o modelo GARCH gaussiano é o mais inapropriado, pois suas caudas leves não conseguem lidar com os valores extremos. O modelo GAS $t$-Student, em geral, apresenta melhores estatísticas que o modelo GARCH $t$-Student, o que está de acordo com o que foi argumentado no Capítulo 5 , no que se refere ao modelo GAS conseguir lidar melhor com valores extremos.

\subsection{Aplicação}

Nesta Seção utilizamos o modelo proposto neste trabalho para a avaliação de aspectos práticos em uma série de dados reais. Como o modelo é uma extensão de modelos que são em geral aplicados a dados financeiros envolvendo preços de ativos negociados no mercado de ações, nos utilizaremos de um conjunto de dados desse tipo.

Em alguns trabalhos que avaliam aplicações de modelos com distribuições estáveis a dados reais, as comparações são feitas apenas com modelos normais (por exemplo, Liu e Brorsen (1995) e Rachev et al. (2005)), e não com modelos que utilizam a distribuição $t$-Student. A distribuiçao $t$-Student muitas vezes é adequada para modelar dados de caudas pesadas, e será considerada nesta Seção.

Em um estudo de comparação de modelos para dados de caudas pesadas, Davis e Mikosch (2007) analisaram a série de retornos diários das ações da Amazon. Os autores utilizaram um modelo $\operatorname{GARCH}(1,1)$ com distribuição condicional $t$-Student e também um modelo de volatilidade estocástica.

Trabalharemos com os mesmos dados, mas no período de 16 de maio de 1997 até 5 de dezembro de 2017 (Davis e Mikosch (2007) analisaram até 16 de junho de 2004). O tamanho da série é $T=5.174$. Os preços das ações foram obtidos no site http://finance.yahoo.com. São considerados os preços no fechamento de cada dia, e os retornos são calculados através da primeira diferença do logaritmo da série.

\subsubsection{Análise preliminar dos dados}

A Figura 6.4 mostra a evolução da série ao longo do tempo. Observam-se alguns retornos que a princípio podem ser imaginados como outliers. Além disso, há diversos outros valores que se destacam, que podem ser decorrentes de uma distribuição com cauda pesada. Também observa-se que a variabilidade da série aparentemente não é constante ao longo do tempo, o que indica que um modelo de variância condicional pode ser adequado.

A Figura 6.5(a) mostra a função de autorrelação amostral da série. O gráfico sugere que a série não possui correlação serial, ou seja, não há necessidade de uma modelagem ARIMA. 
A Figura 6.5(b) mostra a função de autocorrelação dos valores do quadrado série. São observados valores baixos, mas persistentes. Em um processo GARCH espera-se que as autocorrelações da série ao quadrado sigam o padrão de um modelo ARMA, e o gráfico indica que tal modelo pode ser adequado.

A Figura 6.6 mostra a função de codiferença normalizada do quadrado da série, com o objetivo de checar se um modelo com distribuição estável pode ser apropriado. O padrão observado é parecido com o da função de autocorrelação, ou seja, valores baixos, mas persistentes. Logo, um modelo com distribuição estável também deve ser considerado.

A Tabela 6.13 apresenta um sumário com algumas estatísticas da série, entre as quais a curtose e o coeficiente de assimetria.

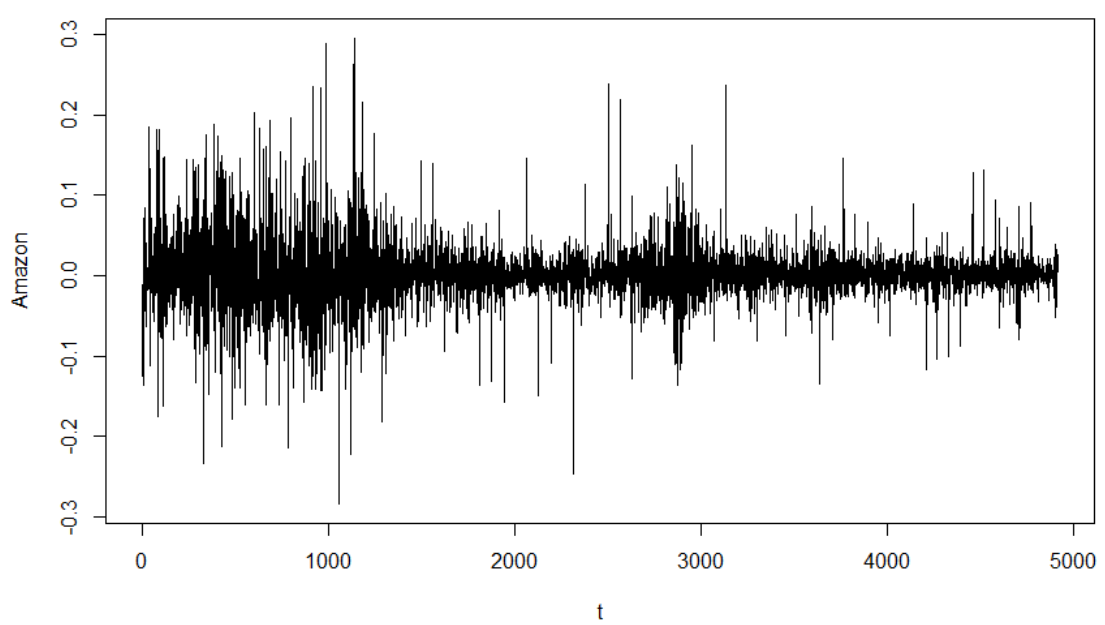

Figura 6.4: Retornos diários das ações da Amazon de 15 de maio de 1997 a 5 de dezembro de 2017 $(T=5.164)$

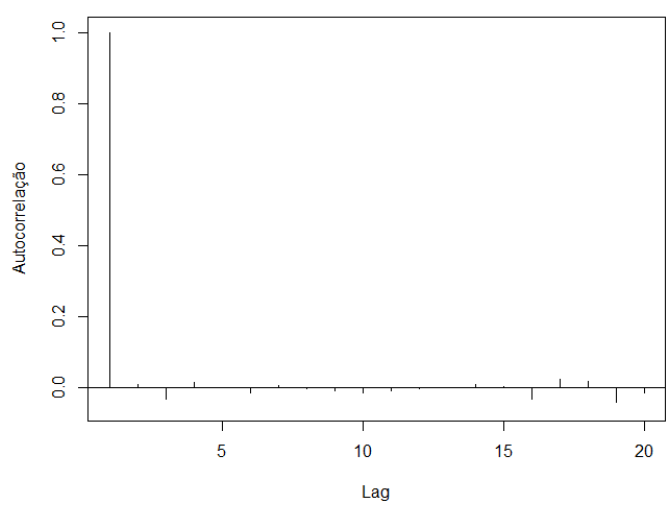

(a) Série original

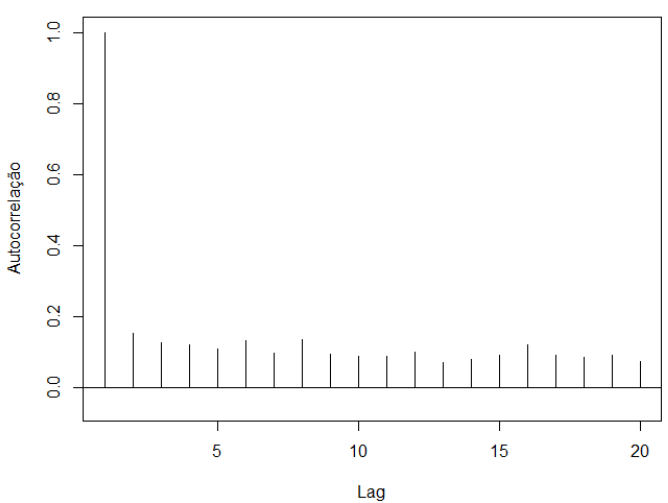

(b) Série ao quadrado

Figura 6.5: Função de autocorrelação amostral da série de retornos diários da Amazon 


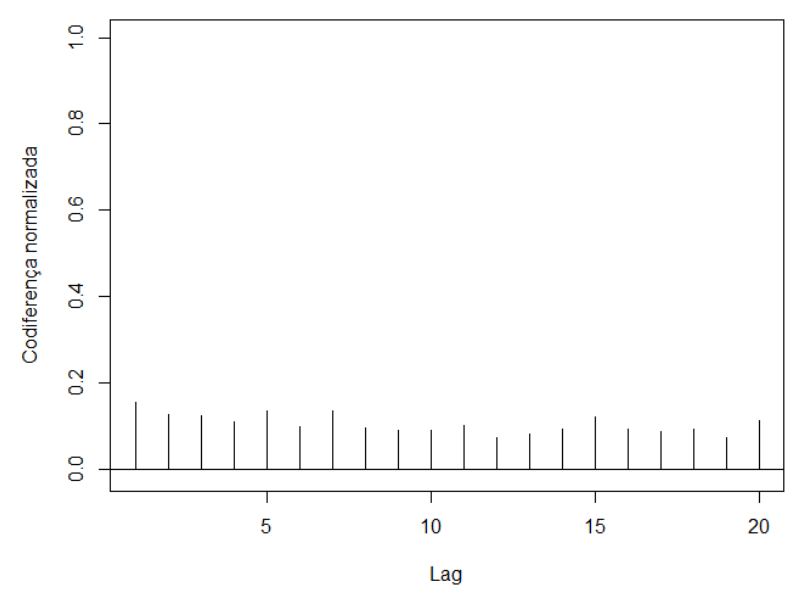

Figura 6.6: Função de codiferença amostral normalizada do quadrado da série de retornos diários da Amazon

Definição 6.2.1 A curtose de uma variável aleatória $X$ é definida como o seu quarto momento padronizado, ou seja,

$$
\operatorname{Curt}(X)=\frac{\mathbb{E}\left[(X-\mathbb{E}[X])^{4}\right]}{\left(\mathbb{E}\left[(X-\mathbb{E}[X])^{2}\right]\right)^{2}}
$$

Uma variável aleatória com distribuição normal tem como valor de curtose 3 . Se $\operatorname{Curt}(X)>3$, é um indicativo de que a distribuição de $X$ tem caudas mais pesadas que as da normal.

Definição 6.2.2 O coeficiente de assimetria de uma variável aleatória $X$ é definida como o seu terceiro momento padronizado, ou seja,

$$
\operatorname{Assim}(X)=\frac{\mathbb{E}\left[(X-\mathbb{E}[X])^{3}\right]}{\left(\mathbb{E}\left[(X-\mathbb{E}[X])^{2}\right]\right)^{3 / 2}}
$$

Uma variável aleatória com coeficiente de assimetria nulo é simétrica.

\begin{tabular}{|c|c|c|c|c|c|c|c|c|}
\hline Média & Mediana & Mín. & Máx. & Desv. padr. & Assim. & Curt. & p-valor AD & p-valor CVM \\
\hline \hline $1,2 \times 10^{-3}$ & 0 & $-0,285$ & 0,296 & 0,039 & 0,443 & 10,798 & $<10^{-4}$ & $<10^{-4}$ \\
\hline
\end{tabular}

Tabela 6.13: Estatísticas descritivas: série de retornos diários da Amazon

Nota-se que a curtose calculada para a série da Amazon é bem maior que a da distribuição normal, que deve ser 3 , indicando caudas pesadas. O coeficiente de assimetria indica que a distribuição dos dados é acentuadamente assimétrica à esquerda (ou assimétrica negativa). Os testes de normalidade empregados (AD e CVM) indicam $p$-valores abaixo de $10^{-4}$, rejeitando-se assim a hipótese nula de normalidade não-condicional. Apesar das observações não serem i.i.d., a análise preliminar indica que a série não é normalmente distribuída.

As estatísticas mencionadas podem ser fortemente influenciadas por valores extremos. A curtose indica caudas pesadas e há indícios de uma leve assimetria à direita. Um modelo de caudas pesadas pode ser indicado não só por causa das características indicadas pela curtose, como também para lidar com os aparentes outliers.

Os testes de qualidade de ajuste também podem ser altamente afetados pelos valores extremos. Por isso, para a análise das estatísticas descritivas, um procedimento foi conduzido para a remoção de outliers. Um modelo GARCH $(1,1)$ gaussiano foi ajustado aos dados. Foi avaliado o maior resíduo em valor absoluto, e a observação correspondente a tal resíduo foi substituída pelo valor médio dos dados. Novamente um $\operatorname{GARCH}(1,1)$ gaussiano foi ajustado. O procedimento foi repetido até que nenhum resíduo apresentasse valor absoluto maior que 3,3 e, assim, teoricamente não houvesse nenhum outlier restante, ao menos considerando-se distribuição normal. 
O novo conjunto de dados apresenta coeficiente de assimetria de 0,154, o que indica uma leve assimetria positiva. A curtose, de 7,546, ainda indica uma distribuição leptocúrtica. Os testes de normalidade indicam $p$-valores abaixo de $10^{-4}$. Ou seja, mesmo com a remoção dos outliers, os dados não parecem ter uma distribuição normal.

Tal análise também deve ser feita aos resíduos após o ajuste do modelo. Mesmo com a remoção dos outliers, os testes de normalidade para os resíduos do modelo $\operatorname{GARCH}(1,1)$ gaussiano indicam $p$-valores abaixo de $10^{-4}$.

A rejeição da hipótese de normalidade em séries de retornos financeiros foi discutida por Zivot (2009), que ressalta que, mesmo após a retirada de outliers, o conjunto restante continua apresentando características de não normalidade.

\subsubsection{Modelagem}

Nesta Seção, será feita a comparação de diferentes modelos aos retornos diários da Amazon, a saber: GARCH gaussiano, GARCH $t$-Student, GARCH estável, GAS $t$-Student e GAS estável.

Para a seleção da ordem do modelo, não é vantajoso, do ponto de vista de previsão de parâmetros futuros, escolher um modelo com ordem alta. Morettin (2011) sugere o uso de modelos de ordem baixa, como $(1,1),(1,2),(2,1)$ ou $(2,2)$, e depois que se escolha o modelo com base em vários critérios, como AIC e BIC. Zivot (2009) concluiu que, na prática, o modelo GARCH(1,1) é dificilmente superado.

No trabalho de Davis e Mikosch (2007), foi utilizado o modelo GARCH(1,1) $t$-Student para a série da Amazon. Aqui, uma análise preliminar (omitida) utilizando as ordens $(1,1),(1,2),(2,1)$ e $(2,2)$ indica que a ordem $(1,1)$, tanto com distribuição condicional normal quanto $t$-Student, é a mais adequada, com base no AICc e no BIC. Tal modelo será utilizado como base para comparação com os outros modelos, para os quais será adotada a mesma ordem.

\begin{tabular}{|c|c|c|c|c|c|}
\hline Parâmetro & $\begin{array}{c}\text { GARCH } \\
\text { gaussiano }\end{array}$ & $\begin{array}{c}\text { GARCH } \\
\text { t-Student }\end{array}$ & $\begin{array}{c}\text { GARCH } \\
\text { estável }\end{array}$ & $\begin{array}{c}\text { GAS } \\
\text {-Student }\end{array}$ & $\begin{array}{c}\text { GAS } \\
\text { estável }\end{array}$ \\
\hline \hline$\omega$ & $1,333 \times 10^{-6}$ & $6,462 \times 10^{-7}$ & $2,770 \times 10^{-5}$ & $-0,249$ & $1,200 \times 10^{-6}$ \\
$A_{1}$ & 0,012 & 0,024 & 0,027 & 0,569 & 0,042 \\
$B_{1}$ & 0,987 & 0,976 & 0,962 & 0,963 & 0,999 \\
$\nu$ & - & 3,780 & - & 24,308 & - \\
$\alpha$ & - & - & 1,744 & - & 1,754 \\
\hline
\end{tabular}

Tabela 6.14: Estimativas dos parâmetros dos modelos ajustados para a série de retornos diários da Amazon

A Tabela 6.14 exibe os valores dos parâmetros estimados para os cinco modelos considerados. A notação para os parâmetros é a mesma que foi utilizada no Capítulo 5.

Com base nos erros padrão das estimativas, todos os valores são significativos ao nível de 0,05 . As exceções são os parâmetros $\omega, B_{1}$ e $\nu$ (graus de liberdade) do modelo GAS $t$-Student, cujos valores dos erros padrão não puderam ser calculados pelos pacotes computacionais correspondentes.

No caso do modelo GARCH gaussiano, observa-se que a soma dos parâmetros $A_{1}$ e $B_{1}$ é muito próxima de 1, indicando uma situação próxima de não estacionariedade. Tal situação de um modelo GARCH gaussiano estimado próximo do limite de estacionariedade é frequentemente encontrada na prática, muitas vezes devido a caudas pesadas da distribuição condicional (Mittnik e Paolella, 2000). É o que ocorre aqui. O fato do modelo gaussiano não conseguir lidar com caudas pesadas de maneira adequada pode ter gerado problemas no cálculo do erro padrão do parâmetro $\omega$.

No caso do modelo GAS $t$-Student, os resultados indicam algo de estranho. O número de graus de liberdade, 24,308, está em total desacordo com o número de 3,78 estimado pelo modelo GARCH $t$-Student. Com tamanho número de graus de liberdade, a distribuição $t$-Student é muito próxima da normal, e aparentemente este não é o caso nesta aplicação. Como o pacote também não conseguiu calcular os erros padrão da maioria dos parâmetros, algum problema de convergência deve ter ocorrido. 


\begin{tabular}{|c|c|c|c|c|c|}
\hline Estatistica & $\begin{array}{c}\text { GARCH } \\
\text { gaussiano }\end{array}$ & $\begin{array}{c}\text { GARCH } \\
\text { t-Student }\end{array}$ & $\begin{array}{c}\text { GARCH } \\
\text { estável }\end{array}$ & $\begin{array}{c}\text { GAS } \\
\text { t-Student }\end{array}$ & $\begin{array}{c}\text { GAS } \\
\text { estável }\end{array}$ \\
\hline \hline Log-verossimilhança & $10.635,00$ & $11.277,88$ & $11.339,46$ & $10.583,64$ & $11.356,60$ \\
AICc & $-21.261,99$ & $-22.545,75$ & $-22.668,91$ & $-21.157,27$ & $-22.703,19$ \\
BIC & $21.244,35$ & $-22.521,56$ & $-22.644,72$ & $-21.133,08$ & $-22.679,00$ \\
Assimetria dos resíduos & 0,422 & 0,762 & 1,153 & 1,307 & 1,323 \\
Curtose dos resíduos & 16,205 & 20,588 & 23,332 & 20,919 & 24,334 \\
p-valor AD para os resíduos & $<10^{-6}$ & 0,004 & 0,004 & $<10^{-6}$ & 0,004 \\
p-valor CMV para os resíduos & $<10^{-6}$ & 0,016 & 0,017 & $<10^{-6}$ & 0,016 \\
MAE para previsão $(\times 50)$ & 0,303 & 0,285 & 0,135 & 0,459 & 0,136 \\
\hline
\end{tabular}

Tabela 6.15: Estatísticas de ajustes dos modelos ajustados para a série de retornos diários da Amazon

A Tabela 6.15 apresenta as estatísticas obtidas com os ajustes dos modelos, para fins de comparação.

O modelo GAS estável apresenta melhor desempenho em termos da log-verossimilhança. No entanto, é adequado avaliar a performance dos modelos em termos de estatísticas que levem em conta o número de parâmetros, como o AICc e o BIC.

O modelo GARCH gaussiano possui três parâmetros estáticos, e os outros três modelos possuem quatro cada um. O AICc e o BIC indicam que o modelo com melhor desempenho é o GAS estável.

A curtose dos resíduos indica valores acima de 15 para todos os modelos, o que justifica a não adequação de um modelo gaussiano e a necessidade de distribuições com caudas pesadas. Todos os modelos apresentam assimetria dos resíduos à direita, o que pode justificar a introdução de um parâmetro de assimetria.

Os testes de qualidade de ajuste AD e CVM indicam que os resíduos se adequam melhor aos modelos com distribuições estáveis (GARCH e GAS) e ao modelo GARCH $t$-Student. No entanto, nenhum $p$-valor apresenta valor acima de 0,05 .

Também foi feita uma comparação com base nas previsões da volatilidade através de "janelas" móveis e reestimação dos modelos, pelo mesmo procedimento utilizado nas simulações. Aqui, inicialmente os modelos são estimados com as primeiras $T-1.000=4.164$ observações, e previsões para $\sigma_{t}$ são geradas para as 20 observações seguintes. Isso resulta no primeiro conjunto de previsão. Para cada conjunto de previsões subsequentes, a amostra utilizada para estimação do modelo tem início e fim com 20 observações depois. Logo, o número de conjuntos de previsões gerado é 50 (1.000 dividido por 20).

As previsões são calculadas de acordo com a estrutura de cada modelo, de acordo com o descrito nas equações $(5.3),(5.8),(5.13)$ e (5.18). As comparações com o valor correspondente amostral do período (que pode ser a variância, no caso dos modelos normal e $t$-Student, ou o parâmetro de escala, no caso dos modelos que utilizam distribuições estáveis) são feitas através do MAE.

As comparações indicam melhores resultados para os modelos com distribuições estáveis, com leve vantagem para o GARCH. No entanto, o $p$-valor do teste Diebold-Mariano, que testa diferença de níveis de acurácia para previsões de diferentes modelos, é de 0,638. Dessa forma, não há evidências de diferenças de performance preditiva entre os modelos GARCH e GAS estáveis.

As Figuras 6.7 a 6.10 exibem a evolução do parâmetro $\sigma_{t}$ e os gráficos de probabilidade dos resíduos para todos os modelos ajustados, com exceção do modelo GAS $(1,1)$ t-Student, por ter apresentado resultados estranhos por possível problema de convergência. Nota-se que os resíduos dos modelos com distribuições estáveis se aproximam mais de uma reta nos gráficos de probabilidade em relação aos resíduos dos outros modelos, indicando melhor adequação às distribuições. 


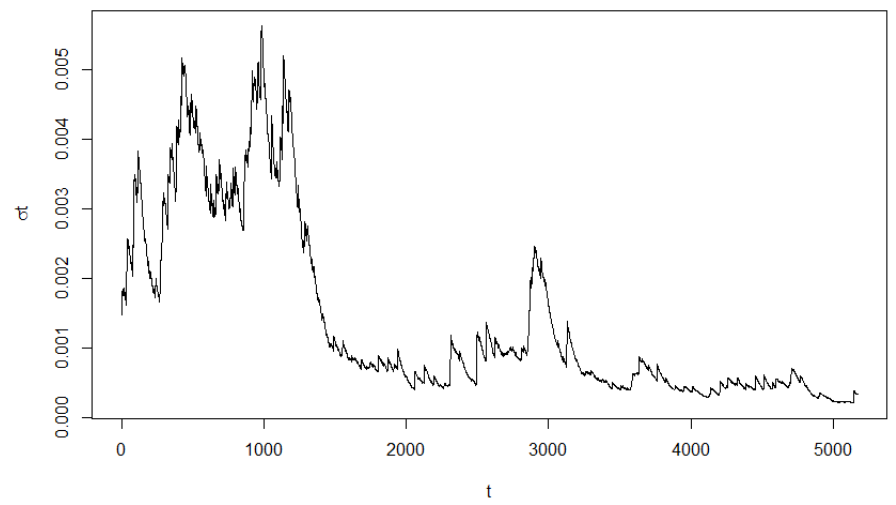

(a) Evolução de $\sigma_{t}$

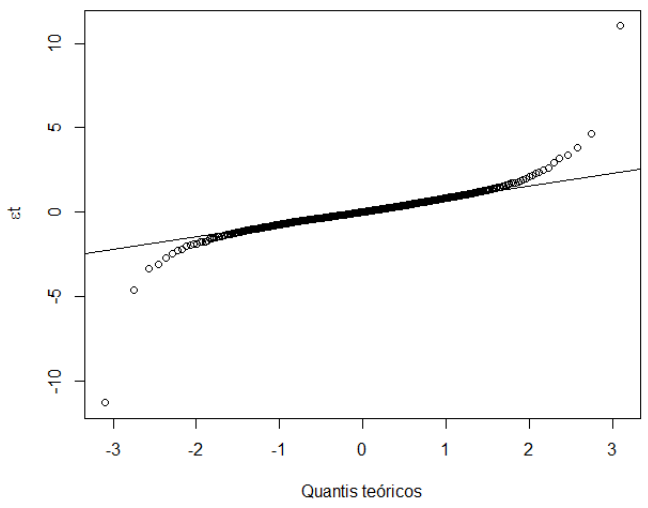

(b) Gráfico de probabilidade dos resíduos

Figura 6.7: Gráficos resultantes do ajuste do modelo $G A R C H(1,1)$ gaussiano para a série de retornos diários da Amazon

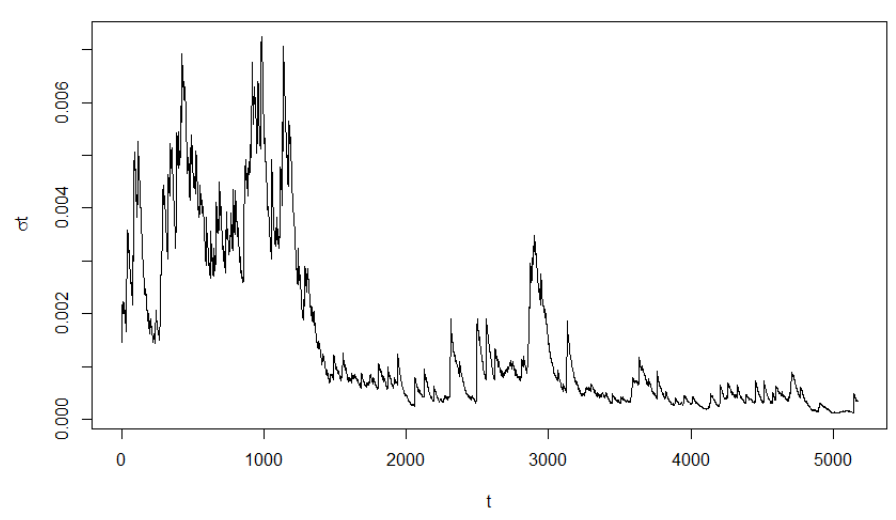

(a) Evolução de $\sigma_{t}$

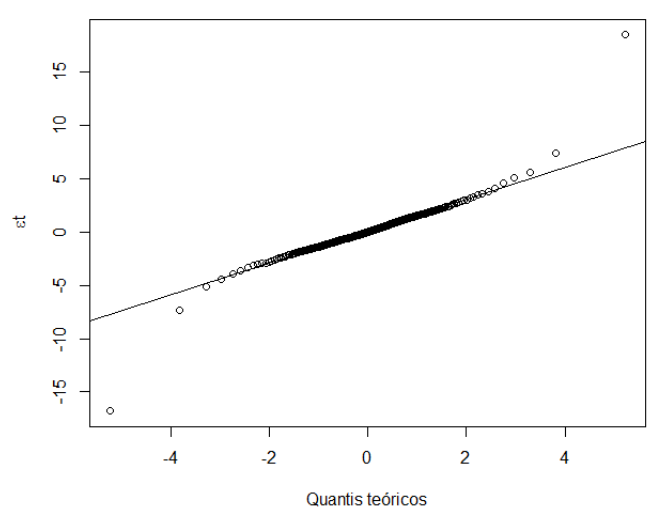

(b) Gráfico de probabilidade dos resíduos

Figura 6.8: Gráficos resultantes do ajuste do modelo $G A R C H(1,1)$ t-Student para a série de retornos diários da Amazon

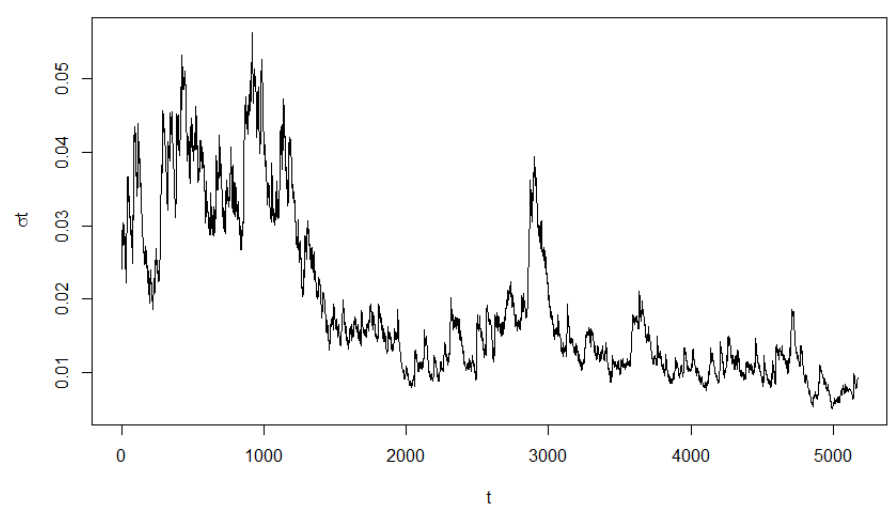

(a) Evolução de $\sigma_{t}$

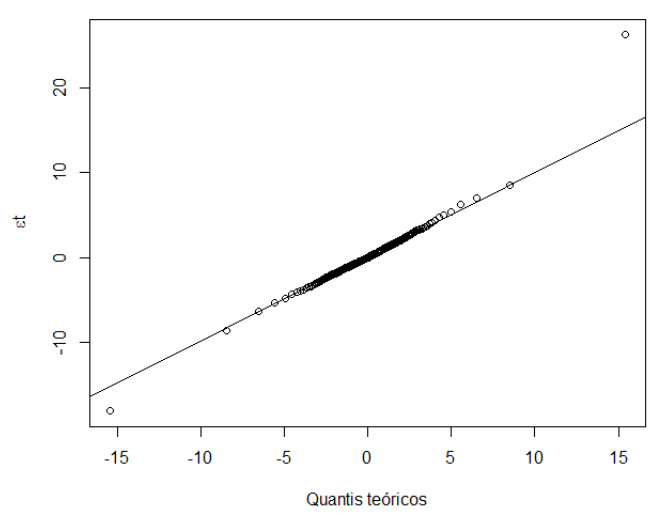

(b) Gráfico de probabilidade dos resíduos

Figura 6.9: Gráficos resultantes do ajuste do modelo $G A R C H(1,1)$ estável para a série de retornos diários da Amazon 


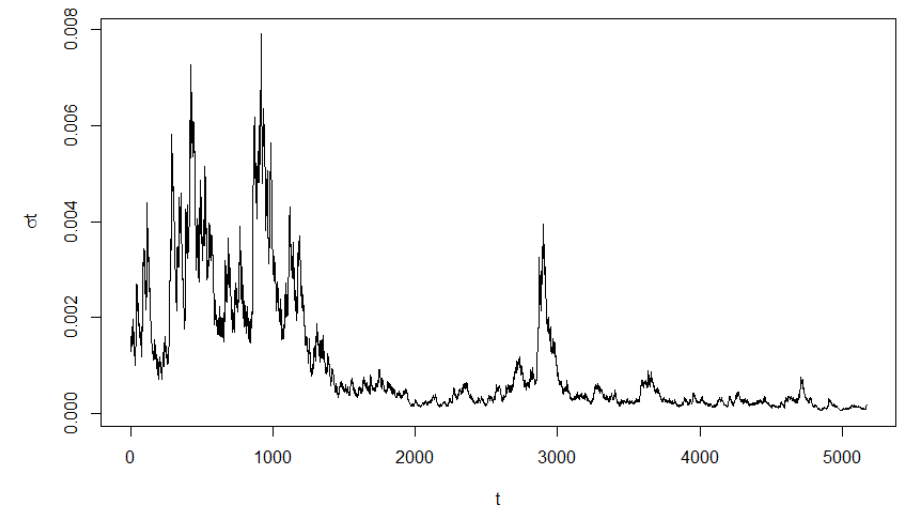

(a) Evolução de $\sigma_{t}$

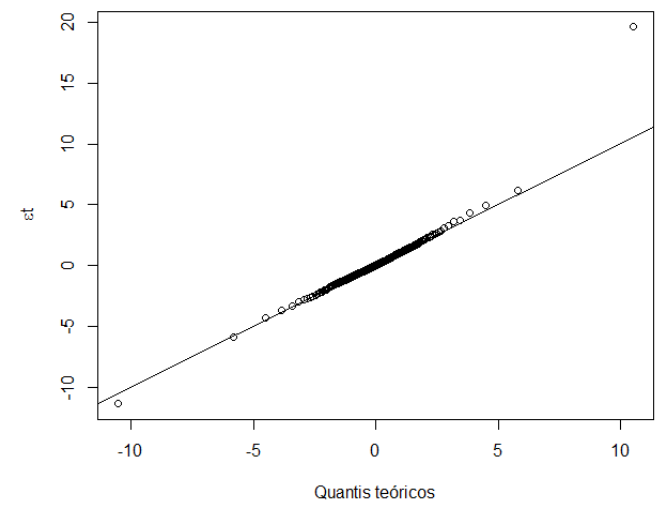

(b) Gráfico de probabilidade dos resíduos

Figura 6.10: Gráficos resultantes do ajuste do modelo $G A S(1,1)$ estável para a série de retornos diários da Amazon

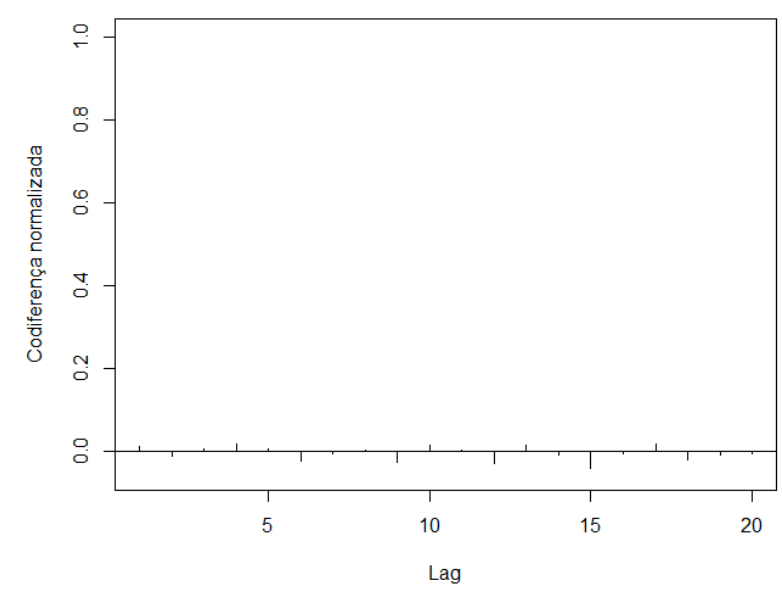

Figura 6.11: Função de codiferença normalizada dos resíduos do modelo GAS(1,1) estável

Espera-se que os resíduos não apresentem estrutura de dependência. A Figura 6.11 exibe a função de codiferença normalizada amostral do modelo $\operatorname{GAS}(1,1)$ estável. De fato, os valores são próximos de zero, indicando fraca estrutura de dependência.

Observação: Para os modelos GARCH e GAS estáveis, foram consideradas distribuições simétricas, ou seja, o parâmetro de assimetria das distribuições estáveis correspondentes foi considerado nulo. No entanto, o pacote GEVStableGarch do R permite que se ajuste um modelo GARCH estável considerando parâmetro de assimetria não nulo para a distribuição estável. A presença de tal parâmetro no modelo pode ser útil, visto que, em todas as análises, os resíduos apresentam indícios de assimetria. De fato, em um $\operatorname{GARCH}(1,1)$ estável com parâmetro de assimetria, o valor estimado do parâmetro é significativo $(0,2$ com erro padrão de 0,0005$)$. O modelo passa a ter log-verossimilhança de 11.346,05, o que resulta em AICc de -22.682, 09 e BIC de -22.657, 90. Ou seja, valores melhores do que os obtidos para o modelo GARCH estável com parâmetro de assimetria nulo. Uma grande melhoria é observada nos testes de qualidade de ajuste. Os $p$-valores dos testes $\operatorname{AD}(0,545)$ e CVM $(0,546)$ são bem mais elevados que os observados nos modelos GARCH e GAS estáveis considerados anteriormente. Ou seja, se uma melhora notável foi observada pela consideração de um parâmetro de assimetria no modelo GARCH estável, é de se esperar também uma melhora se tal parâmetro for considerado em um modelo GAS estável. Esse é um possível tema para trabalhos futuros. 


\section{Capítulo 7}

\section{Conclusões e trabalhos futuros}

Neste Capítulo será revisado o conteúdo apresentado neste trabalho. Também serão fornecidas algumas sugestões para trabalhos futuros e melhoria do modelo proposto.

\subsection{Considerações finais}

Dois fatores comumente presentes em séries econômicas e financeiras são a heteroscedasticidade condicional, ou seja, variância variante ao longo do tempo, e distribuição de caudas pesadas. O objetivo deste trabalho foi propor um modelo que consiga lidar com esses dois aspectos de maneira adequada, e que eventualmente seja mais indicado que os modelos clássicos quando os dados apresentem características específicas.

A consideração de parâmetros dinâmicos em séries temporais é feita há algum tempo e em diversos casos é essencial para uma análise mais adequada de problemas de ordem prática, visto que muitos processos apresentam comportamento variante no tempo. Modelos das classes ARCH e GARCH, utilizados para modelagem de volatilidade, estão entre os mais utilizados para análise de dados financeiros.

Os modelos GAS são modelos dinâmicos guiados por observações que modelam a evolução de parâmetros da distribuição dos dados, que não são constantes ao longo do tempo. Têm a vantagem de se utilizarem da estrutura completa da densidade preditiva do modelo, e não apenas de média ou momentos de ordem superior. Pelo fato da função score ponderada ser utilizada na equação de evolução dos parâmetros dinâmicos da distribuição dos dados, tal equação pode ser interpretada como um algoritmo de subida mais acentuada, se assemelhando ao clássico algoritmo de NewtonRaphson. Além disso, tal estrutura possui propriedades ótimas em termos de minimizar a divergência de Kullback-Leibler em relação à verdadeira densidade dos dados. O clássico modelo GARCH com distribuição condicional normal, para modelagem de volatilidade, é um caso particular de modelo GAS.

Para lidar com caudas pesadas de dados financeiros, a distribuição $t$-Student é uma alternativa à distribuição normal. No entanto, alguns pesquisadores apontaram que a classe de distribuições estáveis pode ser adequada em diversas aplicações, pois possui diversas características atrativas: possibilidade de regulagem do peso das caudas e assimetria; a soma de variáveis aleatórias com distribuições estáveis também tem distribuição estável, o que ocorre com a normal mas não com a $t$-Student (de fato, a normal é um caso particular de distribuição estável); distribuições estáveis possuem domínios de atração e fornecem boas aproximações para um grande número de distribuições. Além disso, em alguns casos práticos, há evidências de que, por causa das caudas pesadas, a distribuição dos dados não possuem segundo momento finito, característica presente nas distribuições estáveis, mas não na $t$-Student.

O modelo proposto, GAS com distribuição estável simétrica para modelagem de volatilidade, foi desenvolvido. O fato de as distribuições estáveis não possuírem funções densidade de probabilidade em forma analítica fechada é um fator complicador, mas é possível proceder com os cálculos explorando a inversão da função característica, que sempre existe, e expansões. Foram verificadas 
restrições para os parâmetros estáticos do modelo para positividade da volatilidade, medida pelo parâmetro de escala da distribuição dos dados, e para estacionariedade. Também foi desenvolvido o procedimento de estimação, baseado no método de Newton-Raphson, o qual inclui quantidades cujos cálculos não aparecem na literatura, como a terceira derivada de uma densidade de uma distribuição estável.

Nos estudos de simulação, foi verificado, em diversos cenários, que o modelo consegue lidar bem com outliers, na maioria das vezes de maneira mais satisfatória que modelos que utilizam a distribuição $t$-Student. Na aplicação a dados reais, o modelo proposto também apresentou bons resultados.

\section{2 $\quad$ Sugestões para trabalhos futuros}

A seguir são indicados alguns aspectos não considerados neste trabalho e que podem ser desenvolvidos:

- avaliação de um modelo ARMA-GAS com distribuição estável, que pode oferecer um ganho de performance em caso de séries temporais com correlação serial;

- consideração de modelos GAS com distribuições estáveis assimétricas. No Capítulo 6 foram encontradas evidências que tal modelo pode oferecer um ganho em termos de ajuste;

- estudo das propriedades teóricas da função de codiferença para o modelo proposto. A análise do comportamento teórico de tal função pode ser de grande utilidade para eventual identificação do modelo; e

- estudo de outros métodos de otimização para estimação dos parâmetros. Apesar do método de Newton-Raphson atender aos propósitos do trabalho, outros algoritmos podem ser mais eficientes em termos de tempo computacional. Além disso, o método de Newton-Raphson é muito sensivel ao valor inicial dos parâmetros a serem estimados.

Pelos resultados obtidos e possibilidades de melhorias, acreditamos que a proposta oferece boas perspectivas para futuros trabalhos. 


\section{Apêndice A}

\section{Códigos}

Códigos em $\mathrm{R}$ de programas para cálculos referentes a distribuições estáveis e estimação dos parâmetros do modelo GAS.

\section{A.1 Densidades de distribuições estáveis e suas derivadas}

\section{Funções auxiliares}

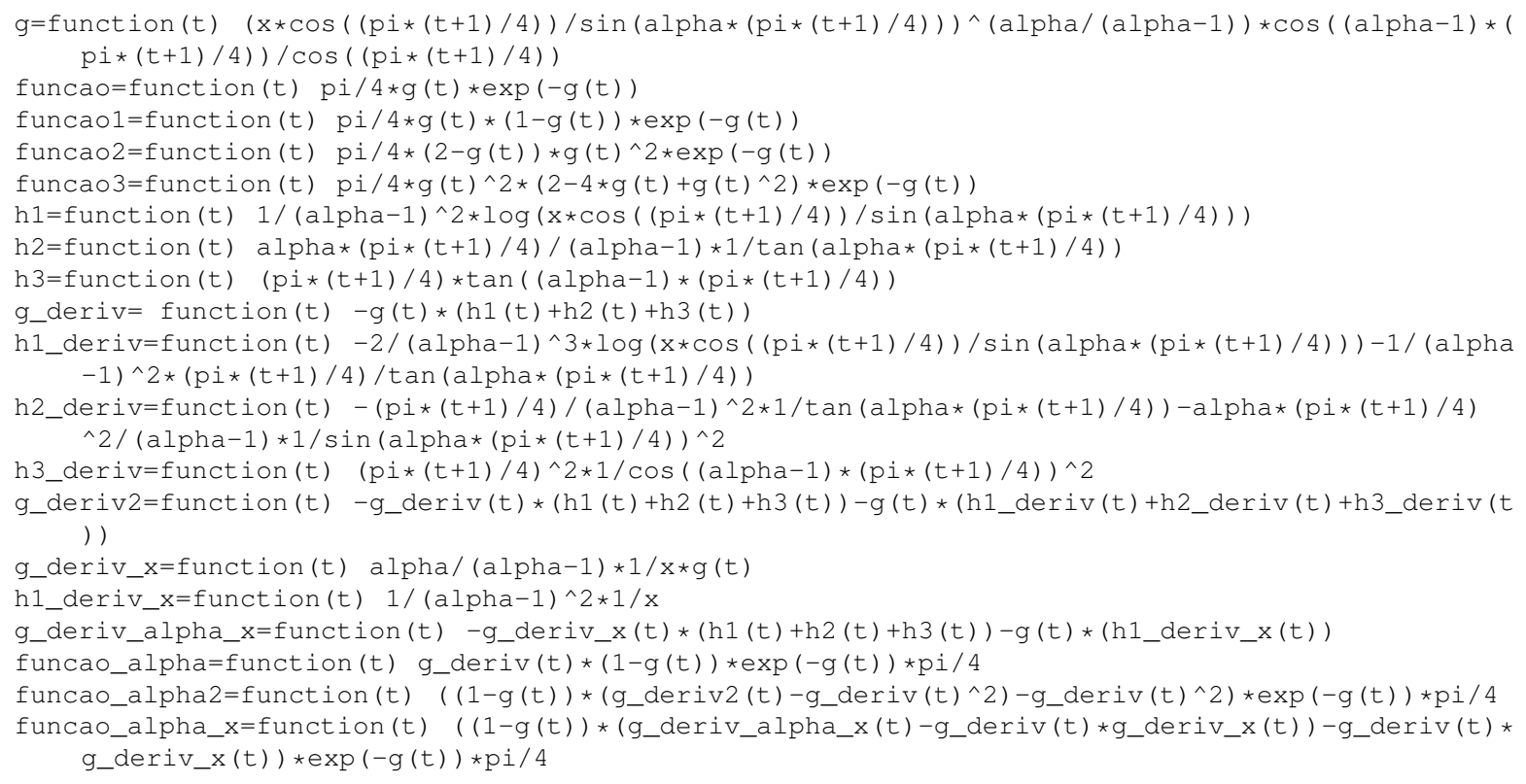

Função para cálculo da densidade de distribuição estável padrão simétrica em relação a $x$

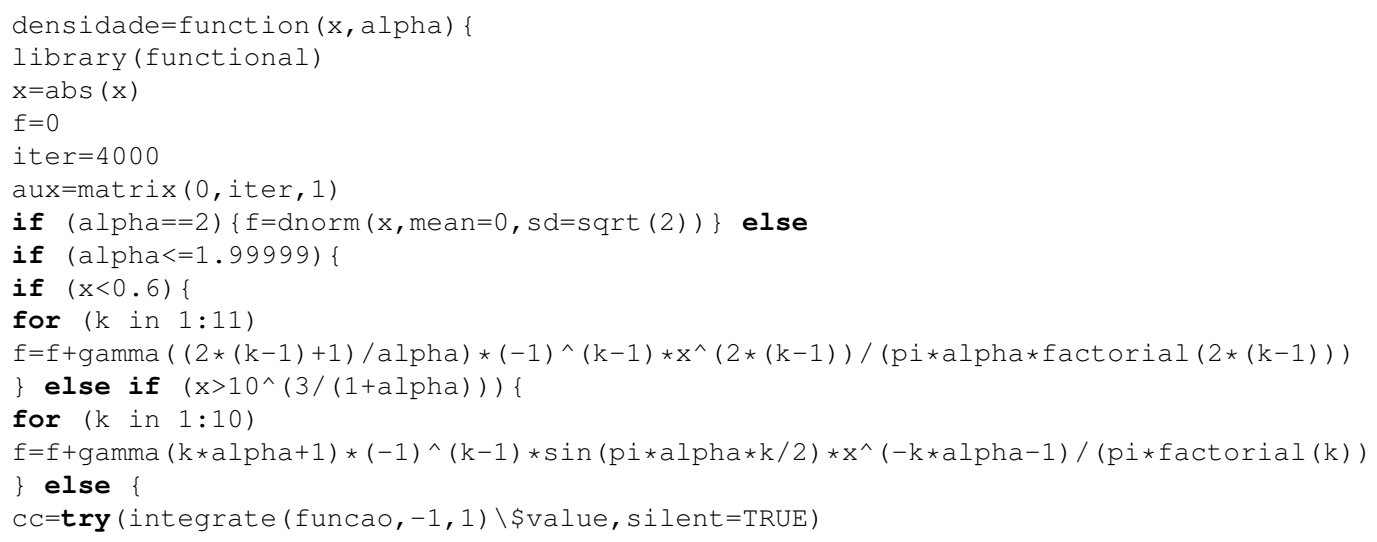




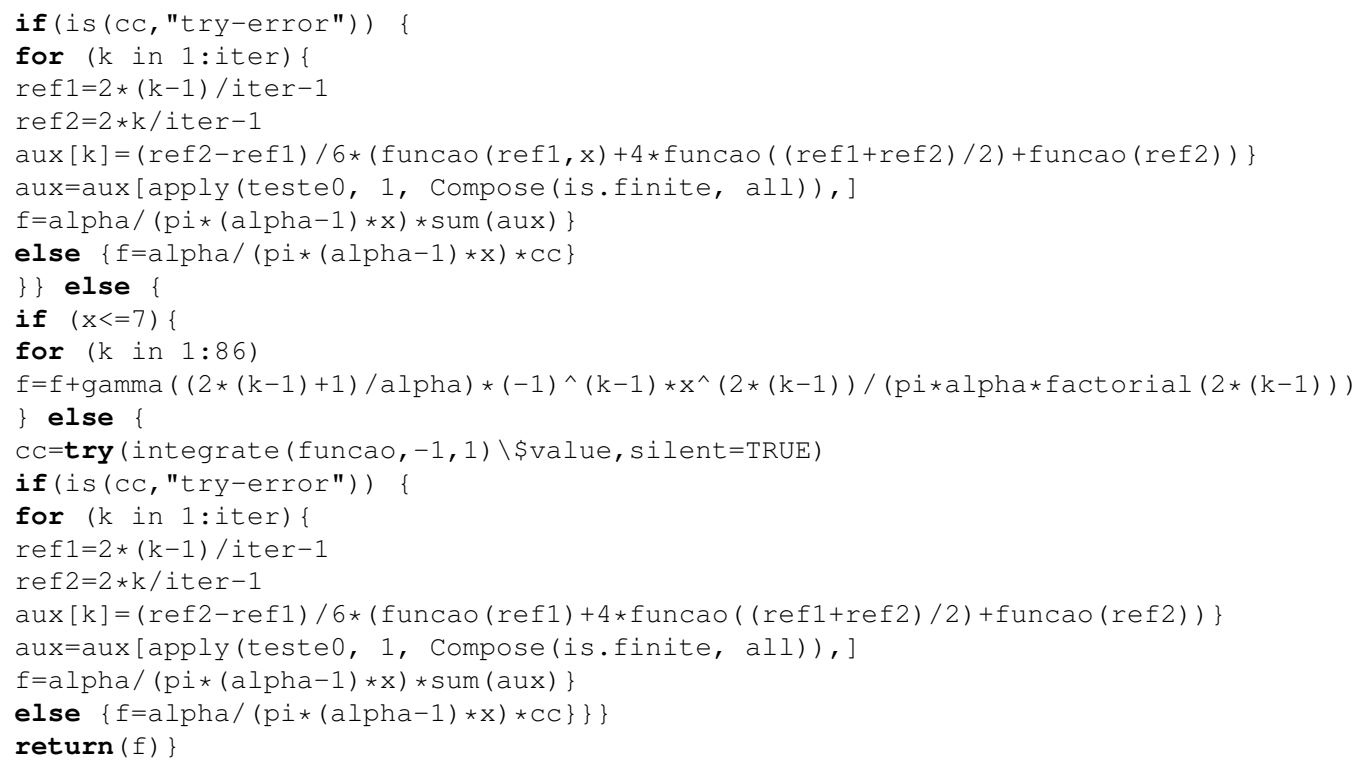

Função para cálculo da primeira derivada da densidade de distribuição estável padrão simétrica em relação a $x$

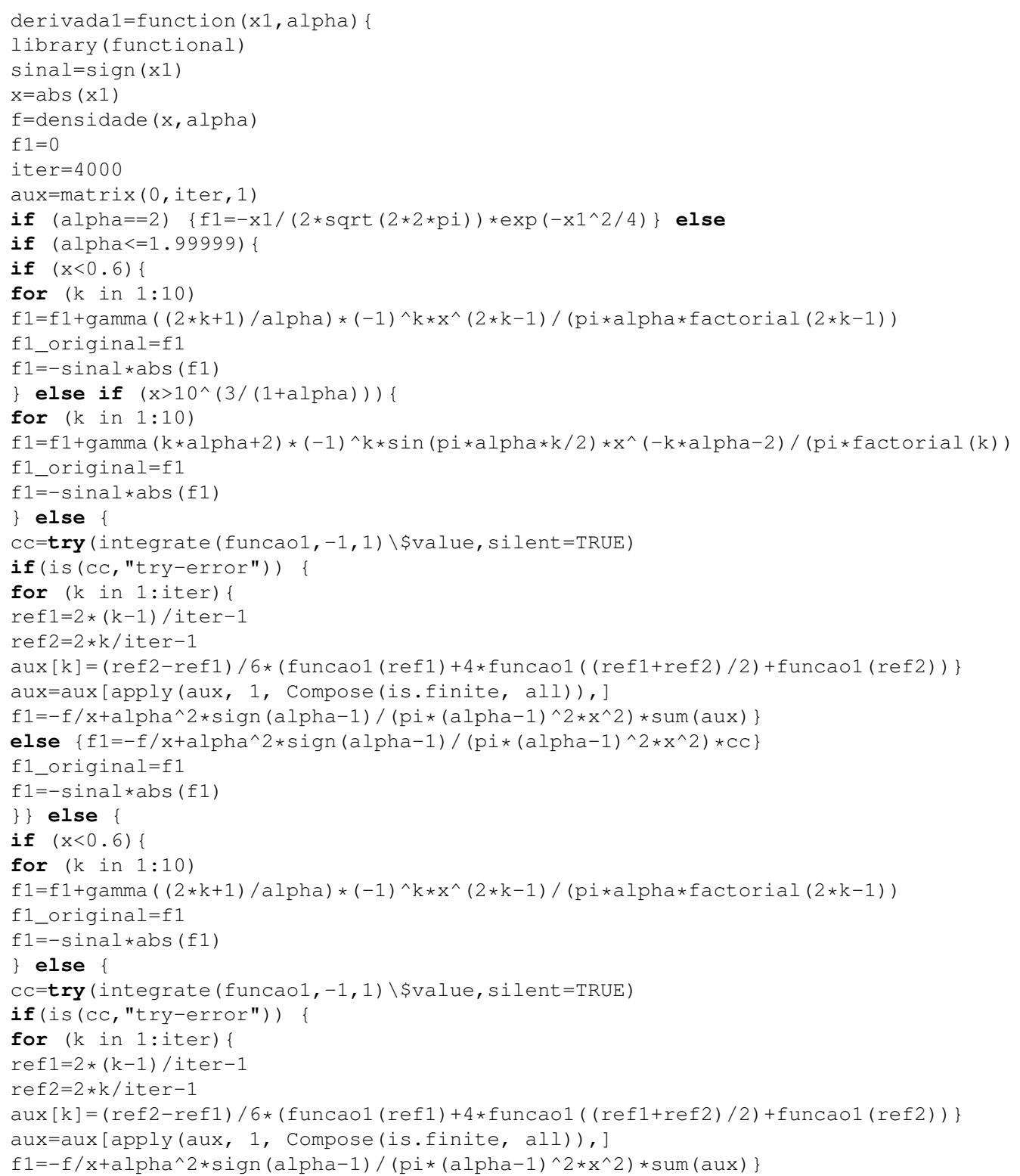


else $\left\{f 1=-f / x+a l p h a \wedge 2 * \operatorname{sign}(a l p h a-1) /\left(p i *(a l p h a-1)^{\wedge} 2 * x^{\wedge} 2\right) * c c\right\}$

$\mathrm{f} 1$ _original $=\mathrm{f} 1$

$\mathrm{f} 1=-\operatorname{sinal} * \mathrm{abs}(\mathrm{f} 1)\}\}$

return $(\mathrm{f} 1)\}$

Função para cálculo da segunda derivada da densidade de distribuição estável padrão simétrica em relação a $x$

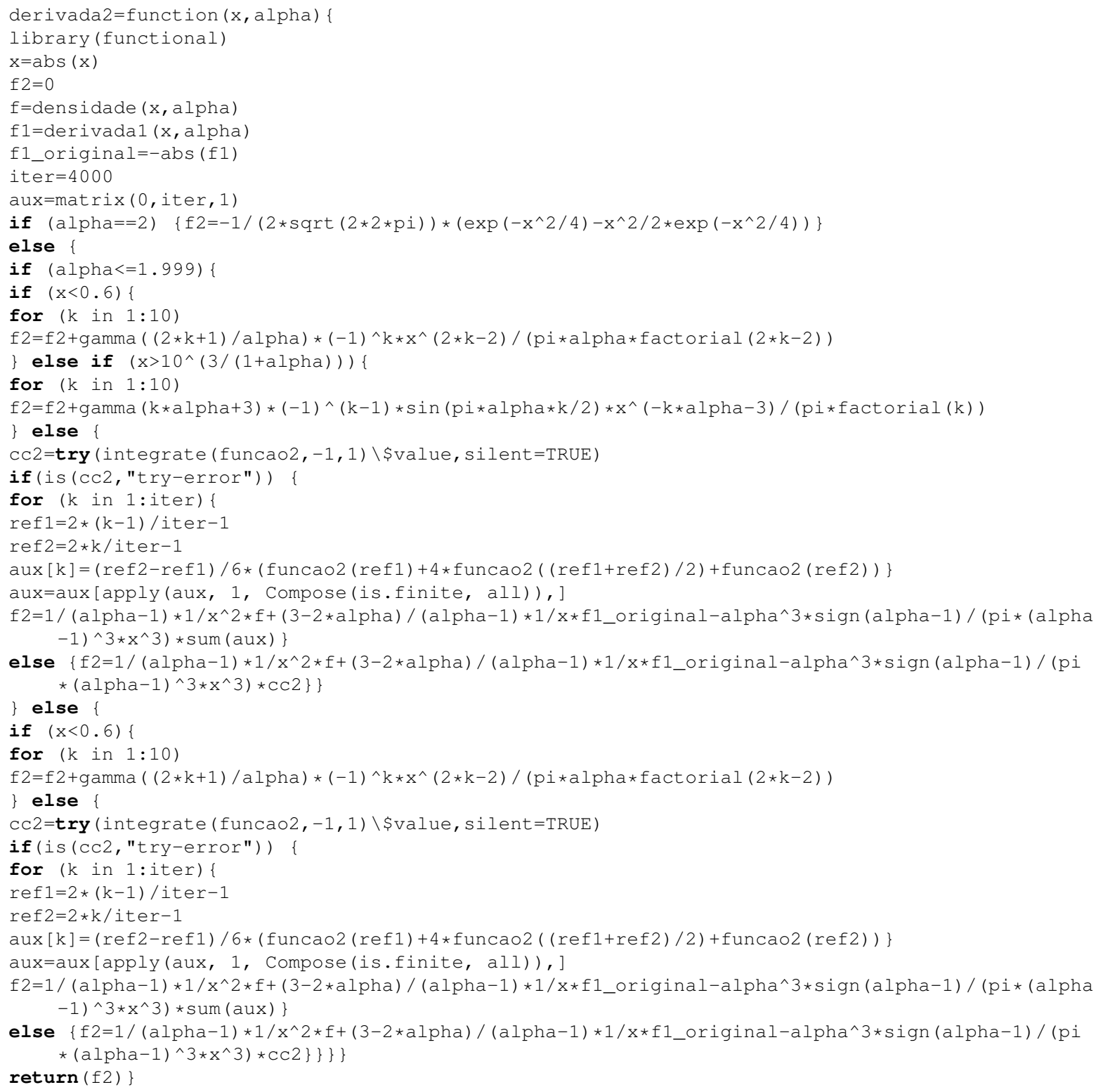

Função para cálculo da terceira derivada da densidade de distribuição estável padrão simétrica em relação a $x$

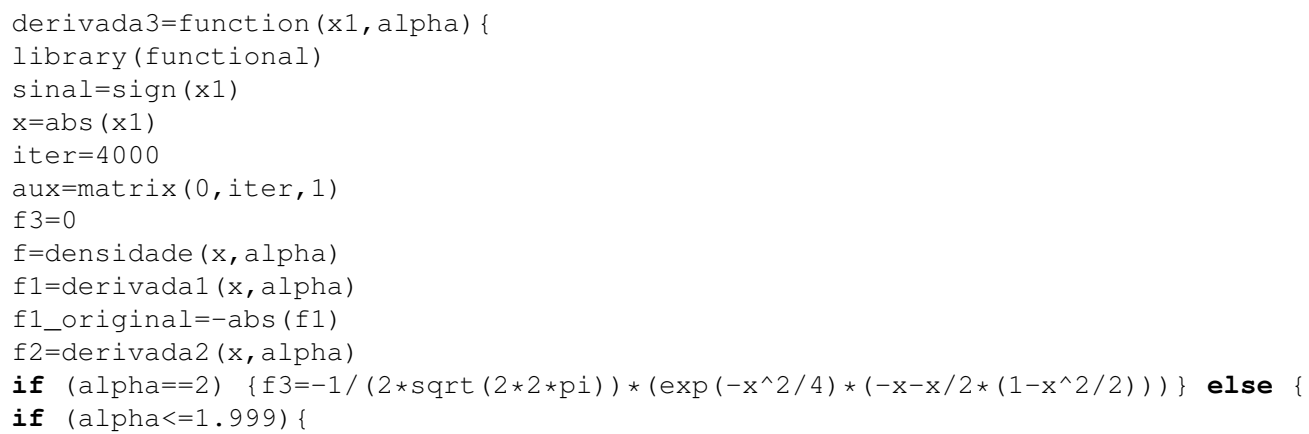


if $(x<0.6)\{$

for $(\mathrm{k}$ in $1: 10)$

$\mathrm{f} 3=\mathrm{f} 3+\mathrm{gamma}((2 * \mathrm{k}+3) / \mathrm{alpha}) *(-1)^{\wedge}(\mathrm{k}+1) * \mathrm{x}^{\wedge}(2 * \mathrm{k}-1) /(\mathrm{pi} * \mathrm{alpha} * \mathrm{factorial}(2 * \mathrm{k}-1))$

\} else if $\left(x>10^{\wedge}(3 /(1+\mathrm{alpha}))\right)\{$

for $(k$ in $1: 10)$

$\mathrm{f} 3=\mathrm{f} 3+\mathrm{gamma}(\mathrm{k} * \mathrm{alpha}+4) *(-1)^{\wedge}(\mathrm{k}) * \sin (\mathrm{pi} * \mathrm{alpha} * \mathrm{k} / 2) * \mathrm{x}^{\wedge}(-\mathrm{k} * \mathrm{alpha}-4) /(\mathrm{pi}$ factorial $(\mathrm{k}))$

\} else \{

cc3=try (integrate ( uncao3,-1,1) \\$value, silent=TRUE)

if (is (cc3, "try-error")) \{

for ( $k$ in 1 :iter) \{

ref $1=2 *(k-1) / i$ ter -1

ref $2=2 * \mathrm{k} /$ iter -1

$\operatorname{aux}[\mathrm{k}]=(\operatorname{ref} 2-\operatorname{ref} 1) / 6 *($ funcao3 $(\operatorname{ref} 1)+4 *$ funcao3 $((\operatorname{ref} 1+\operatorname{ref} 2) / 2)+$ funcao3 $(\operatorname{ref} 2))\}$

aux=aux $[\operatorname{apply}(\operatorname{aux}, 1$, Compose (is.finite, all)), ]

$\mathrm{f} 3=-1 / \mathrm{x}^{\wedge} 3 * 1 /(\mathrm{alpha}-1)^{\wedge} 2 * \mathrm{f}+(-2 * \mathrm{alpha} \wedge 2+8 * \mathrm{alpha}-7) /\left(\mathrm{x}^{\wedge} 2 *(\mathrm{alpha}-1)^{\wedge} 2\right) * \mathrm{f} 1 \_$original $+(6-4 * a l p h a) /(\mathrm{x}$

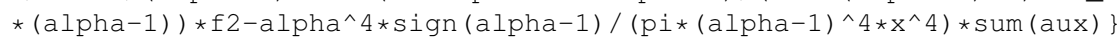

else $\left\{\mathrm{f} 3=-1 / \mathrm{x}^{\wedge} 3 * 1 /(\mathrm{alpha}-1) \wedge 2 * \mathrm{f}+(-2 * \mathrm{alpha} 2+8 * \mathrm{alpha}-7) /\left(\mathrm{x}^{\wedge} 2 *(\mathrm{alpha}-1) \wedge 2\right) * \mathrm{f} 1\right.$ original+(6-4* alpha) / $\left.(x *(a l p h a-1)) * f 2-a l p h a \wedge 4 * \operatorname{sign}(a l p h a-1) /\left(p i *(a l p h a-1)^{\wedge} 4 * x^{\wedge} 4\right) * c c 3\right\}$

\}) else \{

if $(x<0.6)\{$

for $(\mathrm{k}$ in $1: 10)$

$\mathrm{f} 3=\mathrm{f} 3+\mathrm{gamma}((2 * \mathrm{k}+3) / \mathrm{alpha}) *(-1)^{\wedge}(\mathrm{k}+1) * \mathrm{x}^{\wedge}(2 * \mathrm{k}-1) /(\mathrm{pi} * \mathrm{alpha} *$ factorial $(2 * \mathrm{k}-1))$

\} else \{

cc3=try (integrate (funcao3, $-1,1) \backslash$ \$value, silent=TRUE)

if (is (cc3, "try-error")) \{

for ( $\mathrm{k}$ in 1 : iter) \{

ref $1=2 *(k-1) /$ iter -1

ref $2=2 * \mathrm{k} /$ iter -1

$\operatorname{aux}[\mathrm{k}]=(\operatorname{ref} 2-\operatorname{ref} 1) / 6 *($ funcao3 $(\operatorname{ref} 1)+4 *$ funcao3 $((\operatorname{ref} 1+\operatorname{ref} 2) / 2)+$ funcao3 $(\operatorname{ref} 2))\}$

aux=aux [apply (aux, 1, Compose (is.finite, all)), ]

$\mathrm{f} 3=-1 / \mathrm{x}^{\wedge} 3 * 1 /(\mathrm{alpha}-1)^{\wedge} 2 * \mathrm{f}+(-2 * \mathrm{alpha} \wedge 2+8 * \mathrm{alpha}-7) /\left(\mathrm{x}^{\wedge} 2 *(\mathrm{alpha}-1) \wedge 2\right) * \mathrm{f} 1 \_$original $+(6-4 * a l p h a) /(\mathrm{x}$

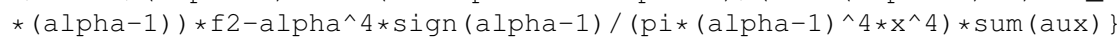

else $\left\{\mathrm{f} 3=-1 / \mathrm{x}^{\wedge} 3 * 1 /(\mathrm{alpha}-1)^{\wedge} 2 * \mathrm{f}+(-2 * \mathrm{alpha} 2+8 * \mathrm{alpha}-7) /\left(\mathrm{x}^{\wedge} 2 *(\mathrm{alpha}-1) \wedge 2\right) * \mathrm{f} 1 \_\right.$original+(6-4* $\left.\left.\left.\left.\mathrm{alpha}) /(\mathrm{x} *(\mathrm{alpha}-1)) * \mathrm{f} 2-\mathrm{alpha} \wedge 4 * \operatorname{sign}(\mathrm{alpha}-1) /\left(\mathrm{pi} *(\mathrm{alpha}-1)^{\wedge} 4 * \mathrm{x}^{\wedge} 4\right) * \mathrm{cc} 3\right\}\right\}\right\}\right\}$

if $(\operatorname{sinal}<0) \quad \mathrm{f} 3=-\mathrm{f} 3$

return (f3) \}

Função para cálculo da primeira derivada da densidade de distribuição estável padrão simétrica em relação a $\alpha$

derivada1_alpha=function ( $x$, alpha) \{

library (functional)

$\mathrm{x}=\mathrm{abs}(\mathrm{x})$

f1_alpha $=0$

$\mathrm{f}=$ densidade $(\mathrm{x}, \mathrm{alpha})$

iter $=4000$

aux=matrix $(0$, iter, 1$)$

if (alpha $<=1.9999)\{$

if $(x<0.6)$

for $(\mathrm{k}$ in $1: 11)$

f1_alpha=f1_alpha- $(\operatorname{gamma}((2 *(\mathrm{k}-1)+1) / \mathrm{alpha})-\mathrm{gamma}((2 *(\mathrm{k}-1)+1) / \mathrm{alpha}) * \mathrm{digamma}((2 *(\mathrm{k}-1)+1) /$ alpha $) *(-2 *(k-1)-1) /$ alpha $) *(-1)^{\wedge}(\mathrm{k}-1) * \mathrm{x}^{\wedge}(2 *(\mathrm{k}-1)) /(\mathrm{pi} * \mathrm{alpha} 2 *$ factorial $(2 *(\mathrm{k}-1)))$

\} else if $\left(x>10^{\wedge}(3 /(1+\mathrm{alpha}))\right)\{$

for $(k$ in $1: 10)$

f1_alpha $=f 1$ _alpha+gamma $(\mathrm{k} * \mathrm{alpha}+1) *(-1) \wedge(\mathrm{k}-1) *\left(\right.$ digamma $(\mathrm{k} * \mathrm{alpha}+1) * \mathrm{k} * \mathrm{sin}(\mathrm{pi} * \mathrm{k} * \mathrm{alpha} / 2) * \mathrm{x}^{\wedge}(-\mathrm{k} *$ $\mathrm{alpha}-1)+\cos (\mathrm{p} i * \mathrm{k} * \mathrm{alpha} / 2) * \mathrm{pi} * \mathrm{k} / 2 * \mathrm{x}^{\wedge}(-\mathrm{k} * \mathrm{alpha}-1)+\sin (\mathrm{pi} * \mathrm{k} * \mathrm{alpha} / 2) * \log (\mathrm{x}) * \mathrm{x}^{\wedge}(-\mathrm{k} * \mathrm{al} \mathrm{pha}-1)$ $*(-\mathrm{k})) /(\mathrm{pi} *$ factorial $(\mathrm{k}))$

\} else \{

cc_alpha=try (integrate (funcao_alpha, -1,1) \\$value, silent=TRUE)

if (is (cc_alpha, "try-error")) \{

for $(k$ in 1 :iter) \{

ref $1=2 *(k-1) /$ iter -1

ref $2=2 * k /$ iter -1

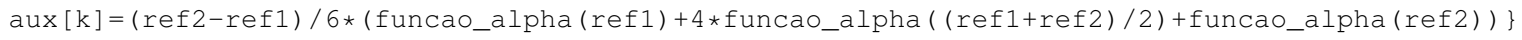

aux=aux [apply (aux, 1, Compose (is.finite, all)), ]

cc_alpha=sum (aux)

$\mathrm{fl}$ _alpha $=-1 /(\mathrm{alpha} *(\mathrm{alpha}-1)) * \mathrm{f}+\mathrm{alpha} /(\mathrm{pi} *(\mathrm{alpha}-1) * \mathrm{x}) * \mathrm{CC} \_\mathrm{alpha}$

else $\left\{f 1 \_a l p h a=-1 /(a l p h a *(a l p h a-1)) * f+a l p h a /(p i *(a l p h a-1) * x) * c c \_a l p h a\right\}$

\}) else \{

if $(x<=8)\{$

for $(k$ in $1: 86)$

f1_alpha=f1_alpha- $(\operatorname{gamma}((2 *(\mathrm{k}-1)+1) / \mathrm{alpha})-\operatorname{gamma}((2 *(\mathrm{k}-1)+1) / \mathrm{alpha}) * \mathrm{digamma}((2 *(\mathrm{k}-1)+1) /$ alpha $) *(-2 *(k-1)-1) /$ alpha $) *(-1)^{\wedge}(\mathrm{k}-1) * x^{\wedge}(2 *(\mathrm{k}-1)) /(\mathrm{pi} * \mathrm{alpha} 2 *$ factorial $(2 *(\mathrm{k}-1)))$

\} else \{ 
for $(k$ in $1: 20)$

f1_alpha=f1_alpha+gamma $(\mathrm{k} * \mathrm{alpha}+1) *(-1)^{\wedge}(\mathrm{k}-1) *(\mathrm{digamma}(\mathrm{k} * \mathrm{alpha}+1) * \mathrm{k} * \mathrm{alpha} * \mathrm{sin}(\mathrm{pi} * \mathrm{k} * \mathrm{alpha} / 2) * \mathrm{x}$ $\wedge(-\mathrm{k} * \mathrm{alpha}-1) * \cos (\mathrm{pi} * \mathrm{k} * \mathrm{alpha} / 2) * \mathrm{pi} * \mathrm{k} / 2 * \mathrm{x}^{\wedge}(-\mathrm{k} * \mathrm{alpha}-1)+\sin (\mathrm{p} i * \mathrm{k} * \mathrm{alpha} / 2) *(-\mathrm{k} * \mathrm{alpha}-1) * \mathrm{x}^{\wedge}(-$ $\mathrm{k} * \mathrm{alpha}-2)) /(\mathrm{pi} * \mathrm{factorial}(\mathrm{k}))\}\}$

return (f1_alpha) \}

Função para cálculo da segunda derivada da densidade de distribuição estável padrão simétrica em relação a $\alpha$

derivada2_alpha=function ( $x$, alpha)

library (functional)

$\mathrm{x}=\operatorname{abs}(\mathrm{x})$

iter $=4000$

aux $=\operatorname{matrix}(0$, iter, 1$)$

aux $1=$ matrix $(0$, iter, 1$)$

f2_alpha=0

$\mathrm{f}=$ densidade $(\mathrm{x}, \mathrm{alpha})$

f1_alpha=derivada1_alpha (x, alpha)

if $(\mathrm{alpha}==2)$ \{

if $(x<=8)$

for ( $k$ in $1: 86)$

f2 alpha=f2 alpha+(gamma $((2 *(k-1)+1) /$ alpha $) * 2 / a l p h a-4 *$ digamma $((2 *(k-1)+1) / a l p h a) * g a m m a((2 *(k$ $-1)+1) / \mathrm{alpha}) *(-2 *(\mathrm{k}-1)-1) / \mathrm{alpha} \wedge 2+\mathrm{gamma}((2 *(\mathrm{k}-1)+1) / \mathrm{alpha}) *(\operatorname{digamma}((2 *(\mathrm{k}-1)+1) / \mathrm{alpha})$

$\wedge 2+$ trigamma $((2 *(\mathrm{k}-1)+1) / \mathrm{alpha})) *(-2 *(\mathrm{k}-1)-1) \wedge 2 / \mathrm{alpha} \wedge 3) *(-1)^{\wedge}(\mathrm{k}-1) * \mathrm{x}^{\wedge}(2 *(\mathrm{k}-1)) /(\mathrm{pi} * \mathrm{al} \mathrm{pha}$

$\wedge 2 *$ factorial $(2 *(k-1)))\}$

else \{

for $(k$ in $1: 20)$

f2_alpha=f2_alpha+gamma $(\mathrm{k} * \mathrm{alpha}+1) *(-1) \wedge(\mathrm{k}-1) *(($ digamma $(\mathrm{k} * \mathrm{alpha}+1) \wedge 2+$ trigamma $(\mathrm{k} * \mathrm{alpha}+1)) * \mathrm{k}$ $\wedge 2 * \sin (\mathrm{pi} * \mathrm{alpha} * \mathrm{k} / 2) * \mathrm{x}^{\wedge}(-\mathrm{k} * \mathrm{alpha}-1)+\operatorname{digamma}(\mathrm{k} * \mathrm{alpha}+1) * \cos (\mathrm{pi} * \mathrm{alpha} * \mathrm{k} / 2) * \mathrm{x}^{\wedge}(-\mathrm{k} * \mathrm{alpha}-1) * \mathrm{k}$ $\wedge 2 * \mathrm{pi} / 2-$ digamma $(\mathrm{k} * \mathrm{alpha}+1) * \sin (\mathrm{pi} * \mathrm{alpha} * \mathrm{k} / 2) * \mathrm{x}^{\wedge}(-\mathrm{k} * \mathrm{alpha}-1) * \log (\mathrm{x}) * \mathrm{k}^{\wedge} 2+\mathrm{digamma}(\mathrm{k} * \mathrm{alpha}+1)$ $* \cos (\mathrm{pi} * \mathrm{alpha} * \mathrm{k} / 2) * \mathrm{x}^{\wedge}(-\mathrm{k} * \mathrm{alpha}-1) * \mathrm{pi} * \mathrm{k} \wedge 2 / 2-\sin (\mathrm{pi} * \mathrm{alpha} * \mathrm{k} / 2) * \mathrm{x}^{\wedge}(-\mathrm{k} * \mathrm{alpha}-1) *(\mathrm{p} i * \mathrm{k} / 2) \wedge 2-$ $\cos (\mathrm{pi} * \mathrm{alpha} * \mathrm{k} / 2) * \mathrm{x}^{\wedge}(-\mathrm{k} * \mathrm{alpha}-1) *(\mathrm{p} i * \mathrm{k} / 2) * \log (\mathrm{x}) * \mathrm{k}-\operatorname{digamma}(\mathrm{k} * \mathrm{alpha}+1) * \mathrm{sin}(\mathrm{p} i * a l p h a * \mathrm{k} / 2) * x$ $\wedge(-\mathrm{k} * \mathrm{alpha}-1) * \log (\mathrm{x}) * \mathrm{k}^{\wedge} 2-\cos (\mathrm{pi} * \mathrm{alpha} * \mathrm{k} / 2) * \mathrm{x}^{\wedge}(-\mathrm{k} * \mathrm{alpha}-1) *(\mathrm{pi} * \mathrm{k} / 2) * \log (\mathrm{x}) * \mathrm{k}+\mathrm{sin}(\mathrm{pi} * \mathrm{alpha} *$ $\left.\mathrm{k} / 2) * \log (\mathrm{x})^{\wedge} 2 * \mathrm{x}^{\wedge}(-\mathrm{k} * \mathrm{alpha}-1) * \mathrm{k}^{\wedge} 2\right) /(\mathrm{pi} *$ factorial $\left.(\mathrm{k}))\right\}$

\} else if (alpha<=1.999) \{

if $(x<0.6)\{$

for $(\mathrm{k}$ in $1: 11)$

f2 alpha=f2 alpha+ (gamma $((2 *(k-1)+1) /$ alpha $) * 2 / a l p h a-4 * \operatorname{digamma}((2 *(\mathrm{k}-1)+1) / \mathrm{alpha}) * g a m m a((2 *(\mathrm{k}$ $-1)+1) / \mathrm{alpha}) *(-2 *(\mathrm{k}-1)-1) / \mathrm{alpha} \wedge 2+\mathrm{gamma}((2 *(\mathrm{k}-1)+1) / \mathrm{alpha}) *(\operatorname{digamma}((2 *(\mathrm{k}-1)+1) / \mathrm{alpha})$ $\wedge 2+$ trigamma $((2 *(\mathrm{k}-1)+1) / \mathrm{alpha})) *(-2 *(\mathrm{k}-1)-1) \wedge 2 / \mathrm{alpha} 3) *(-1)^{\wedge}(\mathrm{k}-1) * \mathrm{x}^{\wedge}(2 *(\mathrm{k}-1)) /(\mathrm{pi} * \mathrm{alpha}$ $\wedge 2 *$ factorial $(2 *(k-1)))$

\} else if $\left(x>10^{\wedge}(3 /(1+\mathrm{alpha}))\right)\{$

for $(\mathrm{k}$ in $1: 10)$

f2_alpha=f2_alpha+gamma $(k * a l p h a+1) *(-1)^{\wedge}(k-1) *(($ digamma $(k * a l p h a+1) \wedge 2+$ trigamma $(k * a l p h a+1)) * k$ $\wedge 2 * \sin (\mathrm{pi} * \mathrm{alpha} * \mathrm{k} / 2) * \mathrm{x}^{\wedge}(-\mathrm{k} * \mathrm{alpha}-1)+\operatorname{digamma}(\mathrm{k} * \mathrm{alpha}+1) * \cos (\mathrm{pi} * \mathrm{alpha} * \mathrm{k} / 2) * \mathrm{x}^{\wedge}(-\mathrm{k} * \mathrm{alpha}-1) * \mathrm{k}$ $\wedge 2 * \mathrm{pi} / 2-\operatorname{digamma}(\mathrm{k} * \mathrm{alpha}+1) * \sin (\mathrm{pi} * \mathrm{alpha} * \mathrm{k} / 2) * \mathrm{x}^{\wedge}(-\mathrm{k} * \mathrm{alpha}-1) * \log (\mathrm{x}) * \mathrm{k}^{\wedge} 2+\mathrm{digamma}(\mathrm{k} * \mathrm{alpha}+1)$ $* \cos (\mathrm{pi} * \mathrm{alpha} * \mathrm{k} / 2) * \mathrm{x}^{\wedge}(-\mathrm{k} * \mathrm{alpha}-1) * \mathrm{p} i * \mathrm{k}^{\wedge} 2 / 2-\sin (\mathrm{pi} * \mathrm{alpha} * \mathrm{k} / 2) * \mathrm{x}^{\wedge}(-\mathrm{k} * \mathrm{alpha}-1) *(\mathrm{p} i * \mathrm{k} / 2) \wedge 2-$ $\cos (\mathrm{pi} * \mathrm{alpha} * \mathrm{k} / 2) * \mathrm{x}^{\wedge}(-\mathrm{k} * \mathrm{alpha}-1) *(\mathrm{pi} * \mathrm{k} / 2) * \log (\mathrm{x}) * \mathrm{k}-\operatorname{digamma}(\mathrm{k} * \mathrm{alpha}+1) * \mathrm{sin}(\mathrm{pi} * \mathrm{alpha} * \mathrm{k} / 2) * \mathrm{x}$ $\wedge(-\mathrm{k} * a \operatorname{lpha}-1) * \log (\mathrm{x}) * \mathrm{k}^{\wedge} 2-\cos (\mathrm{pi} * \mathrm{alpha} * \mathrm{k} / 2) * \mathrm{x}^{\wedge}(-\mathrm{k} * \mathrm{alpha}-1) \star(\mathrm{pi} * \mathrm{k} / 2) * \log (\mathrm{x}) \star \mathrm{k}+\mathrm{sin}(\mathrm{p} i \star \mathrm{alpha} *$ $\left.\mathrm{k} / 2) \star \log (\mathrm{x})^{\wedge} 2 * \mathrm{x}^{\wedge}(-\mathrm{k} * \mathrm{alpha}-1) * \mathrm{k}^{\wedge} 2\right) /(\mathrm{pi} *$ factorial $(\mathrm{k}))$

\section{\} else \{}

cC_alpha2=try (integrate (funcao_alpha2, -1, 1) \\$value, silent=TRUE)

if (is (cc_alpha2, "try-error")) \{

for $(\mathrm{k}$ in 1 :iter)

ref $1=2 *(k-1) /$ iter -1

ref $2=2 * \mathrm{k} /$ iter -1

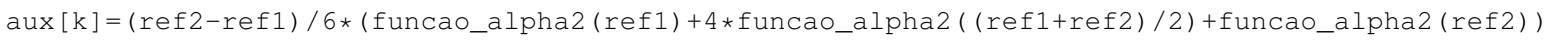
$\operatorname{aux} 1[\mathrm{k}]=(\operatorname{ref} 2-\operatorname{ref} 1) / 6 *\left(\right.$ funcao_alpha $(\operatorname{ref} 1)+4 * f u n c a o$ alpha $\left.\left.((\operatorname{ref} 1+r e f 2) / 2)+f u n c a o \_a l p h a(r e f 2)\right)\right\}$ aux=aux [apply (aux, 1, Compose (is.finite, all)), ]

auxl=aux1 [apply $(\operatorname{auxl}, 1, \operatorname{Compose}($ is.finite, all)), ]

cc_alpha2=sum $($ aux $)$

cC_alpha=sum $($ aux 1$)$

$\mathrm{f} 2$-alpha= $(2 * \mathrm{alpha}-1) * \mathrm{f} /\left((\mathrm{alpha} *(\mathrm{alpha}-1))^{\wedge} 2\right)-\mathrm{f} 1 \_\mathrm{alpha} /(\mathrm{alpha} *(\mathrm{alpha}-1))-1 /(\mathrm{pi} * \mathrm{x} *(\mathrm{alpha}-1) \wedge 2) *$ CC_alpha+alpha/ $(\mathrm{pi} *(\mathrm{alpha}-1) * \mathrm{x}) * \mathrm{cC} \_$_alpha2\}

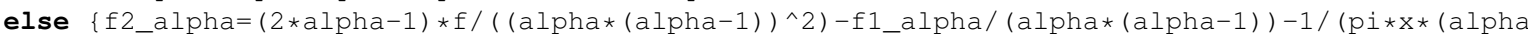
$\left.-1)^{\wedge} 2\right) * \mathrm{cc} \_a l$ pha+alpha/ $(\mathrm{pi} *(\mathrm{alpha}-1) * \mathrm{x}) * \mathrm{cc} \_a l$ pha2

\}) else if $(a \underline{l} p h a<2) \quad\{$

if $(x<=0.6)\{$

for $(\mathrm{k}$ in $1: 11)$

f2_alpha=f2_alpha+(gamma $((2 *(k-1)+1) /$ alpha $) * 2 / a l p h a-4 *$ digamma $((2 *(k-1)+1) / a l p h a) * g a m m a((2 *(k$ $-1)+1) / \mathrm{alpha}) *(-2 *(\mathrm{k}-1)-1) / \mathrm{alpha} \wedge 2+\operatorname{gamma}((2 *(\mathrm{k}-1)+1) / \mathrm{alpha}) *(\operatorname{digamma}((2 *(\mathrm{k}-1)+1) / \mathrm{alpha})$ $\wedge 2+$ trigamma $\left.((2 *(\mathrm{k}-1)+1) / \mathrm{alpha})) *(-2 *(\mathrm{k}-1)-1)^{\wedge} 2 / \mathrm{alpha} \wedge 3\right) *(-1)^{\wedge}(\mathrm{k}-1) * \mathrm{x}^{\wedge}(2 *(\mathrm{k}-1)) /(\mathrm{pi} * \mathrm{alpha}$ $\wedge 2 *$ factorial $(2 *(k-1)))$

\} else \{ 
cc_alpha2=try (integrate (funcao_alpha2, -1, 1) \\$value, silent=TRUE)

if (is (cc_alpha2, "try-error")) \{

for $(k$ in 1 :iter) \{

ref $1=2 *(k-1) /$ iter -1

ref $2=2 * k /$ iter -1

$\operatorname{aux}[\mathrm{k}]=(\operatorname{ref2}-\mathrm{ref1}) / 6 *($ funcao_alpha2 (ref1) $+4 *$ funcao_alpha2 $(($ ref1+ref2) /2) +funcao_alpha2 (ref2)

$\operatorname{aux} 1[\mathrm{k}]=($ ref2-ref1) $/ 6 *($ funcao_alpha $($ refl 1$)+4 *$ funcao_alpha $(($ ref1+ref2)/2) +funcao_alpha (ref2) )

aux=aux [apply (aux, 1, Compose (is.finite, all)), ]

auxl=auxl [apply (aux1, 1, Compose (is.finite, all)), ]

cc_alpha2=sum (aux)

cC_alpha $=\operatorname{sum}(\operatorname{aux} 1)$

$\mathrm{f} 2$ _alpha= $(2 * \mathrm{alpha}-1) * \mathrm{f} /\left((\mathrm{alpha} *(\mathrm{alpha}-1))^{\wedge} 2\right)-\mathrm{f} 1 \_\mathrm{alpha} /(\mathrm{alpha} *(\mathrm{alpha}-1))-1 /(\mathrm{pi} * \mathrm{x} *(\mathrm{alpha}-1) \wedge 2) *$ cC_alpha+alpha/ $(\mathrm{pi} *(\mathrm{alpha}-1) * \mathrm{x}) * \mathrm{c}$ __alpha2

else $\left\{\mathrm{f} 2 \_a l p h a=(2 * a l p h a-1) * f /((a l p h a *(a l p h a-1)) \wedge 2)-f 1 \_a l p h a /(a l p h a *(a l p h a-1))-1 /(p i * x *(a l p h a\right.$

$\left.-1)^{\wedge} 2\right) *$ cc_alpha+alpha/ $\left.\left.\left.(p i *(a l p h a-1) * x) * c c \_a l p h a 2\right\}\right\}\right\}$

return (f2_alpha) \}

\section{Função para cálculo da segunda derivada da densidade de distribuição estável padrão} simétrica em relação a $\alpha$ e a $x$

derivada2_alpha_x=function (x1, alpha) \{

library (functional)

sinal=sign $(x 1)$

$\mathrm{x}=\mathrm{abs}(\mathrm{x} 1)$

f2_alpha_x=0

$\mathrm{fl=derivada1}(\mathrm{x}, \mathrm{alpha})$

f1_original=-abs ( $f 1)$

iter $=4000$

aux=matrix $(0$, iter, 1$)$

$\operatorname{aux} 1=\operatorname{mat} r i x(0$, iter, 1$)$

if $(\mathrm{alpha}==2)\{$

if $(x<=8)\{$

for ( $k$ in 2:86)

f2_alpha_x=f2_alpha_x-(gamma $((2 *(\mathrm{k}-1)+1) / \mathrm{alpha})-\mathrm{gamma}((2 *(\mathrm{k}-1)+1) / \mathrm{alpha}) \star \mathrm{digamma}((2 *(\mathrm{k}-1)+1) /$ alpha $) *(-2 *(k-1)-1) /$ alpha $) *(-1)^{\wedge}(\mathrm{k}-1) *(2 *(\mathrm{k}-1)) * \mathrm{x}^{\wedge}(2 *(\mathrm{k}-1)-1) /(\mathrm{pi} * \mathrm{alpha} 2 * \mathrm{factorial}(2 *(\mathrm{k}$ $-1)$ ))

\} else \{

for $(\mathrm{k}$ in $1: 20)$

f2_alpha_x=f2_alpha_x+gamma $(\mathrm{k} * \mathrm{alpha}+1) *(-1) \wedge(\mathrm{k}-1) * \mathrm{x}^{\wedge}(-\mathrm{k} * \mathrm{alpha}-2) *(\mathrm{digamma}(\mathrm{k} * \mathrm{alpha}+1) * \mathrm{k} * \mathrm{sin}(\mathrm{pi}$ $* \mathrm{k} * \mathrm{alpha} / 2) *(-\mathrm{k} * \mathrm{alpha}-1)+\cos (\mathrm{pi} * \mathrm{k} * \mathrm{alpha} / 2) * \mathrm{pi} * \mathrm{k} / 2 *(-\mathrm{k} * \mathrm{alpha}-1)+\mathrm{sin}(\mathrm{pi} * \mathrm{k} * \mathrm{alpha} / 2) *(-\mathrm{k})+\mathrm{sin}$ $(\mathrm{pi} * \mathrm{k} * \mathrm{alpha} / 2) * \log (\mathrm{x}) *(-\mathrm{k} * \mathrm{alpha}-1) *(-\mathrm{k})) /(\mathrm{pi} *$ factorial $(\mathrm{k}))\}$

\} else if (alpha<=1.999) \{

if $(x<0.6)\{$

for $(k$ in $2: 11)$

f2_alpha_x=f2_alpha_x-(gamma $((2 *(k-1)+1) /$ alpha $)-g a m m a((2 *(k-1)+1) / a l p h a) *$ digamma $((2 *(k-1)+1)$ alpha $) *(-2 *(k-1)-1) / a l p h a) *(-1)^{\wedge}(k-1) *(2 *(k-1)) * x^{\wedge}(2 *(k-1)-1) /(p i * a l p h a \wedge 2 * f a c t o r i a l(2 *(k$ $-1)$ ))

\} else if $\left(x>10^{\wedge}(3 /(1+\mathrm{alpha}))\right)\{$

for $(k$ in $1: 10)$

f2_alpha_x=f2_alpha_x+gamma $(\mathrm{k} * \mathrm{alpha}+1) *(-1)^{\wedge}(\mathrm{k}-1) * \mathrm{x}^{\wedge}(-\mathrm{k} * \mathrm{alpha}-2) *(\mathrm{digamma}(\mathrm{k} * \mathrm{alpha}+1) * \mathrm{k} * \mathrm{sin}(\mathrm{pi}$ $* \mathrm{k} * \mathrm{al} \mathrm{pha} / 2) *(-\mathrm{k} * \mathrm{alpha}-1)+\cos (\mathrm{pi} * \mathrm{k} * \mathrm{alpha} / 2) * \mathrm{pi} * \mathrm{k} / 2 *(-\mathrm{k} * \mathrm{alpha}-1)+\sin (\mathrm{pi} * \mathrm{k} * \mathrm{alpha} / 2) *(-\mathrm{k})+\mathrm{sin}$ $(\mathrm{pi} * \mathrm{k} * \mathrm{alpha} / 2) * \log (\mathrm{x}) *(-\mathrm{k} * \mathrm{alpha}-1) *(-\mathrm{k})) /(\mathrm{pi} * \mathrm{factorial}(\mathrm{k}))$

\} else

cc_alpha_x=try (integrate (funcao_alpha_x, -1,1) \\$value, silent=TRUE)

if (is (cc_alpha_x,"try-error")) \{

for $(\mathrm{k}$ in 1 :iter) \{

ref $1=2 *(k-1) /$ iter -1

ref $2=2 * k /$ iter -1

$\operatorname{aux}[\mathrm{k}]=($ ref2-ref 1$) / 6 *($ funcao_alpha_x (ref1) 4 *funcao_alpha_x((ref1+ref2)/2)+funcao_alpha x ( $r e f 2))$

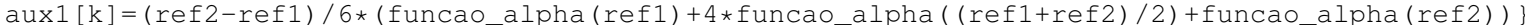

aux=aux [apply $(\operatorname{aux}, 1$, Compose (is.finite, all)), ]

auxl=auxl[apply (auxl, 1, Compose (is.finite, all)), ]

cc_alpha_x=sum (aux)

cC_alpha=sum $(\mathrm{aux} 1)$

f2_alpha_x=-1/(alpha*(alpha-1))*f1_original-alpha/ (pi*(alpha-1)*x^2)*cc_alpha+alpha/ (pi* alpha-1)*x)*cC_alpha_x

else \{f2_alpha_x=-1/(alpha*(alpha-1))*f1_original-alpha/(pi*(alpha-1)*x^2)*cC_alpha+alpha/(pi *(alpha-1)*x)*CC_alpha_x

\}) else if $(a$ lpha $<2)$ \{

if $(x<=0.6)$

for $(k$ in 2:11)

f2_alpha_x=f2_alpha_x-(gamma $((2 *(\mathrm{k}-1)+1) / \mathrm{alpha})-\mathrm{gamma}((2 *(\mathrm{k}-1)+1) / \mathrm{alpha}) \star \mathrm{digamma}((2 *(\mathrm{k}-1)+1) /$ alpha $) *(-2 *(k-1)-1) /$ alpha $) *(-1)^{\wedge}(\mathrm{k}-1) *(2 *(\mathrm{k}-1)) * x^{\wedge}(2 *(\mathrm{k}-1)-1) /(\mathrm{pi} * \mathrm{alpha} 2 *$ factorial $(2 *(\mathrm{k}$ 


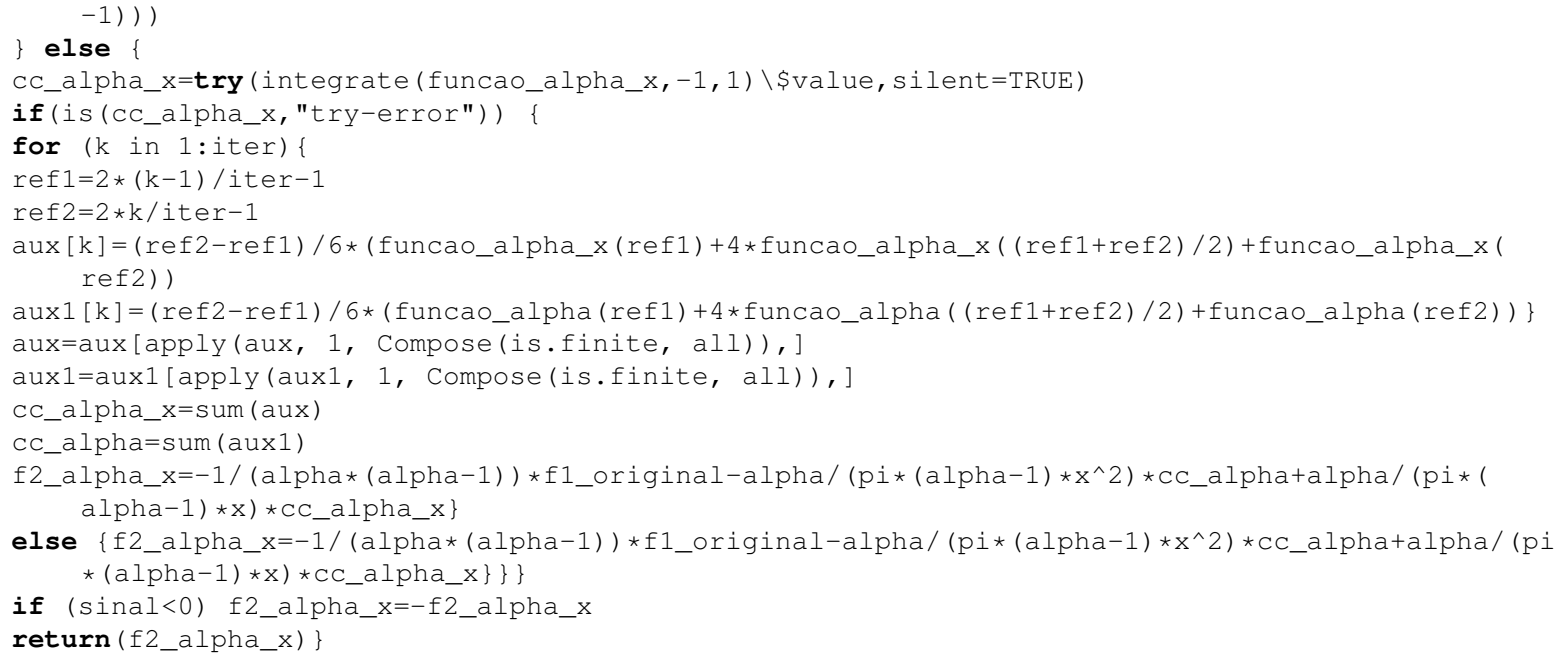

\section{A.2 Informação de Fisher de distribuição estável padrão simétrica em relação ao parâmetro de escala}

Conforme verificado na Seção 4.3, o cálculo da informação de Fisher envolve o cálculo de uma integral numérica. No entanto, a utilização de uma aproximação oferece resultados muito precisos e com economia de tempo computacional.

A Tabela A.1 mostra valores da informação de Fisher $\mathcal{I}^{\text {std }}$ de distribuições simétricas padrão em relação ao parâmetro de escala para diversos valores de $\alpha$ entre 1 e 2, calculados numericamente a partir da expressão (4.57). Os valores estão de acordo com aqueles calculados por Matsui e Takemura (2006). A Figura A.1 exibe o gráfico desses valores, dando uma ideia de como se comporta a relação.

Para o cálculo de $\mathcal{I}^{\text {std }}$ para demais valores de $\alpha$ entre 1 e 2 , uma função foi escrita em $\mathrm{R}$ (funcao_informacao) de modo que funções de segundo grau interpolassem cada três pontos do gráfico. Os valores de tais funções foram então utilizadas para o cálculo dos valores aproximados de $\mathcal{I}^{\text {std }}$. As aproximações são satisfatórias, e nas checagens executadas para diversos valores de $\alpha$ o erro encontrado em valor absoluto em relação o valor verdadeiro não foi maior que $10^{-6}$.

\begin{tabular}{|c|c||c|c|}
\hline$\alpha$ & $\mathcal{I}^{\text {std }}$ & $\alpha$ & $\mathcal{I}^{\text {std }}$ \\
\hline \hline 2 & 2 & 1,55 & 1.0145 \\
1,99 & 1.9321 & 1,5 & 0.9556 \\
1,98 & 1.8819 & 1,45 & 0.9000 \\
1,95 & 1.7631 & 1,4 & 0.8475 \\
1,9 & 1.6127 & 1,35 & 0.7975 \\
1,85 & 1.4922 & 1,3 & 0.7498 \\
1,8 & 1.3898 & 1,25 & 0.7042 \\
1,75 & 1.2997 & 1,2 & 0.6603 \\
1,7 & 1.2189 & 1,15 & 0.6181 \\
1,65 & 1.1453 & 1,1 & 0.5774 \\
1,6 & 1.0775 & 1,05 & 0.5381 \\
\hline
\end{tabular}

Tabela A.1: Valores de $\mathcal{I}^{\text {std }}$ para diferentes valores de $\alpha$ 


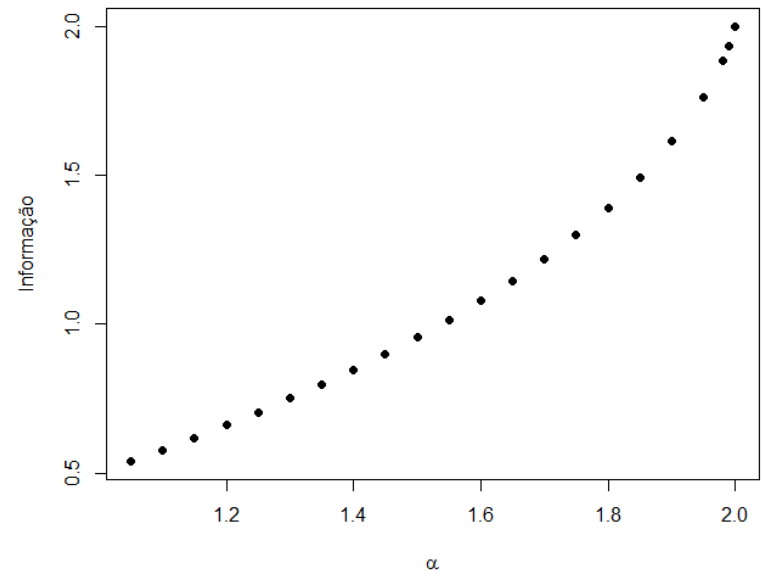

Figura A.1: Gráfico de $\alpha$ por $\mathcal{I}^{\text {std }}$ para $1<\alpha \leq 2$

\section{A.3 Estimação dos parâmetros do modelo GAS com distribuição estável}

Função para estimação dos parâmetros do modelo GAS(1,1) com distribuição estável

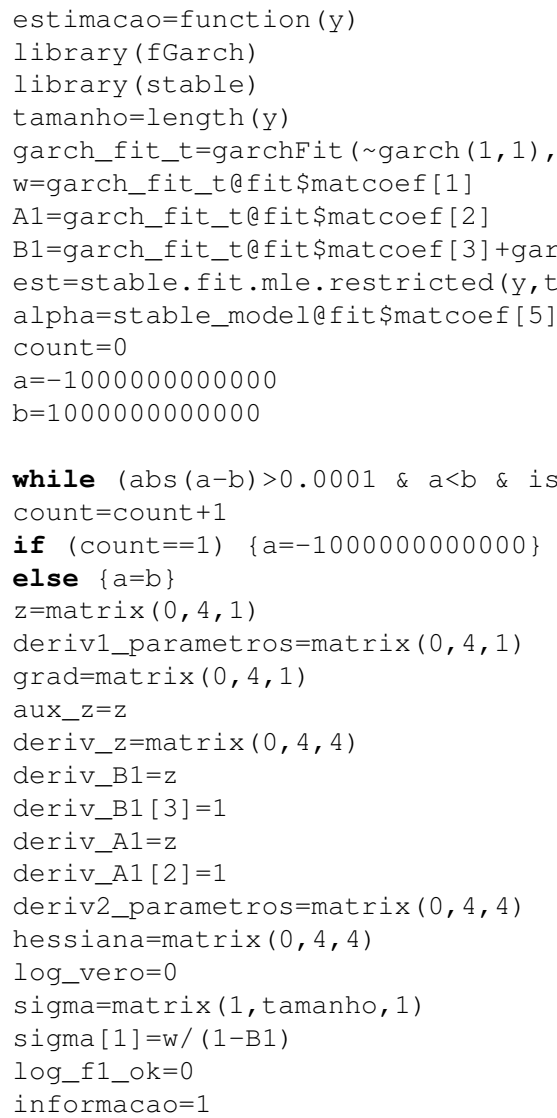


$\mathrm{f} 2=\operatorname{derivada} 2(\mathrm{y}, \mathrm{alpha})$

$\mathrm{f} 3=\operatorname{derivada} 3(\mathrm{y}, \mathrm{alpha})$

f_ok=(1/h_sigma) $* f$

$\mathrm{f} 1$ _ok $=-\mathrm{h} 1$ _sigma/h_sigma^2*f-y[i]*h1_sigma/h_sigma^3*f1

$\mathrm{f} 2$ _ok $=(2 * \mathrm{~h} 1$ _sigma^2-h2_sigma*h_sigma) /h_sigma^3*f+y[i] * (4*h1_sigma^2-h2_sigma*h_sigma)/ h_sigma^4*f1+y[i]^2*h1_sigma^2/h_sigma^5*f2

f3_ok=(6*h_sigma*h1_sigma*h2_sigma-h_sigma^2*h3_sigma-6*h1_sigma^3)/h_sigma^4*f+y[i]*(12*

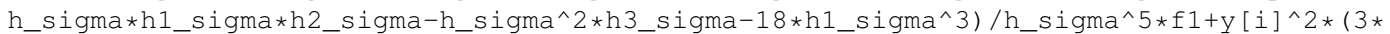

h_sigma*h1_sigma*h2_sigma-9*h1_sigma^3)/h_sigma^6*f2-y[i]^3*h1_sigma^3/h_sigma^7*f3

log_vero=log_vero+log (f_ok)

log_f1_ok=1/f_ok $* \mathrm{f} 1$ _ok

log_f2_ok $=\left(\mathrm{f} 2\right.$ _ok $* \mathrm{f} \_\mathrm{ok}-\mathrm{f} 1$ _ok^2) /f_ok^2

log_f3_ok=(f3_ok*f_ok^2-3*f_ok*f1_ok*f2_ok+2*f1_ok^3) /f_ok^3

f1_alpha=derivada1_alpha (y, alpha)

f2_alpha=derivada2_alpha (y, alpha)

f2_alpha_x=derivada2_alpha_x (y, alpha)

$\mathrm{f} 1$ _alpha_ok $=\left(1 / \mathrm{h} \_\right.$sigma $) * \mathrm{f} 1$ _alpha

f2_alpha_ok=(1/h_sigma) *f2_alpha

informacao=funcao_informacao(alpha) / (4*sigma[i]^2) \}

deriv1_parametros_anterior=deriv1_parametros

deriv2_parametros_anterior=deriv2_parametros

$\mathrm{z}[1]=1$

$z[2]=$ log_deriv1[i-1]/info[i-1]

$z[3]=$ sigma $[i-1]$

$z[4]=0$

deriv1_parametros=z+B1*deriv1_parametros_anterior+A1*deriv1_parametros_anterior*(1og_deriv2 [i

-1]/info[i-1]+log_deriv1[i-1]*2/(info[i-1]*sigma[i-1]))

deriv1_sigma=log_deriv1[i]*deriv1_parametros

deriv1_sigma [4]=f1_alpha/densid_padr[i]

grad=grad+deriv1_sigma

aux_z $[1]=0$

aux_z[2]=log_deriv2[i-1]/info[i-1]+log_deriv1[i-1]*2/(info[i-1]*sigma [i-1])

aux_z $[3]=1$

aux_z $[4]=0$

deriv_z [1, ] =aux_z*deriv1_parametros_anterior[1]

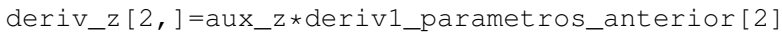

deriv_z [3, ] =aux_z*deriv1_parametros_anterior[3]

deriv_z [4,]=aux_z*deriv1_parametros_anterior[4]

deriv_z=t (deriv_z)

deriv2_parametros=deriv_z+deriv1_parametros_anterior\#*\#t (deriv_B1) +B1*

deriv2_parametros_anterior+

(deriv1_parametros_anterior\#*\#t (deriv_A1) +A1*deriv2_parametros_anterior)*(log_deriv2 [i-1] / info[i-1]+log_deriv1[i-1]*2/(info[i-1]*sigma[i-1]))+

A1*deriv1_parametros_anterior\#*\#t (log_deriv3[i-1]*deriv1_parametros_anterior/info[i-1] +2* log_deriv2 [i-1]*deriv1_parametros_anterior*2/(info[i-1]*sigma[i-1]) +2*

deriv1_parametros_anterior*log_deriv1[i-1]/(info[i-1]*sigma[i-1]^2))

deriv2_sigma=log_deriv2[i]*deriv1_parametros\#*\#t (deriv1_parametros) +log_deriv1[i]* deriv2_parametros

deriv2_sigma $[1,4]=\left(f 2 \_a l p h a \_x * d e n s i d \_p a d r[i]-f 1 \_a l p h a * d e r i v 1 \_p a d r[i]\right) / d e n s i d \_p a d r[i] \wedge 2 *(-y[i$ ] /h_sigma^2)*h1_sigma*deriv1_parametros[1]

deriv2_sigma [2,4]=(f2_alpha_x*densid_padr[i]-f1_alpha*deriv1_padr[i])/densid_padr[i]^2*(-y[i ]/h_sigma^2)*h1_sigma*deriv1_parametros [2]

deriv2_sigma [3,4]=(f2_alpha_x*densid_padr[i]-f1_alpha*deriv1_padr[i])/densid_padr[i]^2*(-y[i ] /h_sigma^2)*h1_sigma*deriv1_parametros [3]

deriv2_sigma $[4,4]=-\left(f 1 \_a l p h a\right) \wedge 2 /$ densid_padr[i]^2+f2_alpha/densid_padr[i]

deriv2_sigma $[4,1]=$ deriv2_sigma $[1,4]$

deriv2_sigma $[4,2]=\operatorname{deriv2}$ _sigma $[2,4]$

deriv2_sigma $[4,3]=\operatorname{deriv2}$ _sigma $[3,4]$

deriv2_sigma $[4,4]=\operatorname{deriv2\_ sigma~}[4,4]$

hessiana=hessiana+deriv2_sigma

\}

sigma_final=sigma

$\mathrm{b}=\operatorname{sum}(\log ($ densid) [2:tamanho])

W_ant $=\mathrm{W}$

A1_ant $=\mathrm{A} 1$

B1_ant $=\mathrm{B} 1$

alpha_ant =alpha

for (s in $1: 20)\{$

$\mathrm{w}=\mathrm{w}$ _ant $-\mathrm{s} / 20 *($ solve (hessiana) \#*\#grad) [1]

$\mathrm{A} 1=\mathrm{A} 1$ _ant $-\mathrm{s} / 20 *$ (solve (hessiana) \#*\#grad) [2]

B1=B1_ant - s/20* (solve (hessiana) \#*\#grad) [3]

alpha=alpha_ant-s/20*(solve (hessiana)\#*\#grad) [4]

if $(\mathrm{alpha}>=2) \quad\{\mathrm{alpha}=2\}$ 
log_vero_aux $=0$

log_f1_ok=0

informacao $=1$

for ( $i$ in 2 :length $(y))\{$

sigma [i]=w+A1*log_f1_ok/informacao+B1*sigma [i-1]

h_sigma=sqrt (sigma [i])/sqrt (2)

h1_sigma=sigma [i]^ $(-1 / 2) /(2 * \operatorname{sqrt}(2))$

$\mathrm{x}=\mathrm{y}[\mathrm{i}] / \mathrm{h} \_\mathrm{sigma}$

$\mathrm{f}=$ densidade $(\mathrm{x}, \mathrm{alpha})$

$\mathrm{f} 1=\operatorname{derivada1}(\mathrm{x}, \mathrm{alpha})$

f_ok $=\left(1 / h \_s i g m a\right) * f$

f1_ok=-h1_sigma/h_sigma^2*f-y[i] *h1_sigma/h_sigma^3*f1

log_f1_ok=1/f_ok*f1_ok \}

log_vero_aux=log_vero_aux+log (f_ok)

informacao=funcao_informacao(alpha) / (4*sigma[i]^2)\}\}

auxiliar $[s, 1]=s / 20$

auxiliar $[s, 2]=w$

auxiliar $[\mathrm{s}, 3]=\mathrm{A} 1$

auxiliar $[\mathrm{s}, 4]=\mathrm{B} 1$

auxiliar $[\mathrm{s}, 5]=$ alpha

auxiliar $[\mathrm{s}, 6]=$ log_vero_aux

auxiliarl=auxiliar [order (auxiliar $[, 6])$, ]

$\mathrm{w}=\operatorname{auxiliar} 1[20,2]$

A1=auxiliarl $[20,3]$

$\mathrm{B} 1=\operatorname{auxiliar1}[20,4]$

alpha=auxiliarl[20,5]

\}) 


\section{Referências Bibliográficas}

Akgiray(1989) Vedat Akgiray. Conditional heteroscedasticity in time series of stock returns: evidence and forecasts. The Journal of Business, 62(1):55-80. Citado na pág.

Ardia et al.(2016) David Ardia, Kris Boudt e Leopoldo Catania. Generalized autoregressive score models in R: the GAS package, 2016. URL https://arxiv.org/pdf/1609.02354.pdf. Último acesso em $05 / 11 / 2017$. Citado na pág. 6

Blasques et al.(2014a) Francisco Blasques, Siem Jan Koopman e Andre Lucas. Stationarity and ergodicity of univariate generalized autoregressive score processes. Electronic Journal of Statistics, 8:1088-1112. Citado na pág. 3, 11, 50

Blasques et al.(2014b) Francisco Blasques, Siem Jan Koopman e Andre Lucas. Maximum likelihood estimation for generalized autoregressive score models. Relatório Técnico TI 2014-029/III, Tinbergen Institute, University of Amsterdam. Citado na pág. 13

Blasques et al.(2015) Francisco Blasques, Siem Jan Koopman e Andre Lucas. Informationtheoretic optimality of observation-driven time series models for continuous responses. Biometrika, 102(2):325-343. Citado na pág. 2, 4, 8, 9, 10, 40, 45

Blasques et al.(2016) Francisco Blasques, Andre Lucas e Erkki Silde. A stochastic recurrence equations approach for score driven correlation models. Econometric Reviews, páginas 1-16. Citado na pág. 7

Bollerslev(1986) Tim Bollerslev. Generalized autoregressive conditional heteroskedasticity. Journal of Econometrics, 31(3):307-327. Citado na pág. 3, 7, 13, 42, 49

Bollerslev(1987) Tim Bollerslev. A conditionally heteroskedastic time series model for speculative prices and rates of return. The Review of Economics and Statistics, 69(3):542-547. Citado na pág. $7,39,42$

Bougerol e Picard(1992) Philippe Bougerol e Nico Picard. Stationarity of GARCH processes and of some nonnegative time series. Journal of Econometrics, 52(1-2):115-127. Citado na pág. 42, $50,52,53$

Brandt(1986) Andreas Brandt. The stochastic equation $Y_{n+1}=A_{n} Y_{n}+B_{n}$ with stationary coefficients. Advances in Applied Probability, 18(1):211-220. Citado na pág. 50

Brant(1984) Rollin Brant. Approximate likelihood and probability calculations based on transforms. Annals of Statistics, 12(3):989-1005. Citado na pág. 26

Buckle(1995) D. J. Buckle. Bayesian inference for stable distributions. Journal of the American Statistical Association, 90(430):605-613. Citado na pág. 15, 16

Carnero et al.(2012) M. Angeles Carnero, Daniel Peña e Esther Ruiz. Estimating GARCH volatility in the presence of outliers. Economic Letters, 114(11):86-90. Citado na pág. 
Chambers et al.(1976) John M. Chambers, Collin L. Mallows e B. W. Stuck. A method for simulating stable random variables. Journal of the American Statistical Association, 71(354): 340-344. Citado na pág. 26

Constantinides et al.(2003) George M. Constantinides, Milton Harris e René M. Stulz. Handbook of the Economics and Finance. Elsevier, Amsterdam. Citado na pág.

Cootner(1964) Paul H. Cootner. Comments on the variation of certain speculative prices (Mandelbrot (1963). Em Paul H. Cootner, editor, The Random Character of Stock Market Prices, páginas 413-418. MIT Press (Reprinted Risk Books(2000)). Citado na pág. 17

Cox(1981) David Roxbee Cox. Statistical analysis of time series: some recent developments. Scandinavian Journal of Statistics, 8(2):93-115. Citado na pág. 3

Creal et al.(2008) Drew Creal, Siem Jan Koopman e Andre Lucas. A general framework for observation driven time-varying parameter models. Relatório Técnico TI 2008-108/4, Tinbergen Institute, University of Amsterdam. Citado na pág. 1, 4, 5

Creal et al.(2011) Drew Creal, Siem Jan Koopman e Andre Lucas. A dynamic multivariate heavy-tailed model for time-varying volatilities and correlations. Journal of Business 85 Economic Statistics, 29(4):552-563. Citado na pág. 7, 41, 50

Creal et al.(2013) Drew Creal, Siem Jan Koopman e Andre Lucas. Generalized autoregressive score models with applications. Journal of Applied Econometrics, 28(5):777-795. Citado na pág. 1, $2,4,5,6,8,41,44$

Davis e Mikosch(2007) Richard A. Davis e Thomas Mikosch. Heavy Tails and Time Series Models. Em SAMSI Risk Workshop, Durham. URL http://www.stat.columbia.edu/ ${ }^{\text {rdavis/ }}$ lectures/SAMSI_07.pdf. Último acesso em 05/11/2017. Citado na pág. 79, 82

de Vries(1991) Casper G. de Vries. On the relation between GARCH and stable processes. Journal of Econometrics, 48(3):313-324. Citado na pág. 39

DuMouchel(1973) William H. DuMouchel. On the asymptotic normality of the maximumlikelihood estimate when sampling from a stable distribution. Annals of Statistics, 1(5):948-957. Citado na pág. 17,26

DuMouchel(1975) William H. DuMouchel. Stable distributions in statistical inference : 2. Information from stably distributed samples. Journal of the American Statistical Association, 70 (350):386-393. Citado na pág. 16, 47

Engle(1982) Robert F. Engle. Autoregressive conditional heteroscedasticity with estimates of the variance of United Kingdom inflation. Econometrica, 50(4):987-1007. Citado na pág. 3, 42

Fama(1963) Eugene F. Fama. Mandelbrot and the stable Paretian hypothesis. The Journal of Business, 36(4):420-429. Citado na pág. 16, 39, 40

Fama(1965) Eugene F. Fama. The behavior of stock-market prices. Journal of Business, 38(1): 34-105. Citado na pág. 16,39

Fama e Roll(1968) Eugene F. Fama e Richard Roll. Some properties of symmetric stable distributions. Journal of the American Statistical Association, 63(323):817-836. Citado na pág. 16, 26

Fama e Roll(1971) Eugene F. Fama e Richard Roll. Parameter estimates for symmetric stable distributions. Journal of the American Statistical Association, 66(334):331-338. Citado na pág. 16, 17 
Figueira Neto(2013) Domingos Theodoro Figueira Neto. Cálculo de VaR para uma Carteira de Ações: Sistema Informatizado para Ações Negociadas na Bovespa, 2013. Trabalho de Conclusão de Curso de Graduação, Instituto de Economia, Universidade Federal do Rio de Janeiro. Citado na pág.

Fiorentini et al.(1996) Gabriele Fiorentini, Giorgio Calzolari e Lorenzo Panattoni. Analytic derivatives and the computation of GARCH estimates. Journal of Applied Econometrics, 11(4): 399-417. Citado na pág. $13,55,56$

Frain(2009) John C. Frain. Studies on the Application of the $\alpha$-stable Distribution in Economics. Tese de Doutorado, Department of Economics, University of Dublin. Citado na pág. 17, 40, 58

Gilbert(2001) William J. Gilbert. Generalizations of Newton's method. Fractals, 9(3):251-262. Citado na pág. 55

Gnedenko e Kolmogorov(1968) Boris Vladimirovich Gnedenko e Andrei Nikolaevich Kolmogorov. Limit Distributions for Sums of Independent Random Variables. Addison-Wesley, Massachusetts. Citado na pág. 15, 17, 23

Harvey(2013) Andrew Harvey. Dynamic Models for Volatility and Heavy Tails: With Applications to Financial and Economic Time Series. Econometric Series Monographs, Cambridge University Press. Citado na pág. 1, 4, 7, 8, 11, 39, 44

Harvey(1989) Andrew Harvey. Forecasting Structural Time Series Models and the Kalman Filter. Cambridge University Press. Citado na pág. 1

Harvey e Chakravarty(2008) Andrew Harvey e Tirthankar Chakravarty. Beta-t-(E)GARCH. Relatório Técnico CWPE 08340. Citado na pág. 1, 4

Harvey e Luati(2014) Andrew Harvey e Alessandra Luati. Filtering with heavy tails. Journal of the American Statistical Association, 109(507):1112-1122. Citado na pág. 7, 41

Hobson(1971) Art Hobson. Concepts in Statistical Mechanics. Gordon \& Breach Publishing Group. Citado na pág. 8

Janus et al.(2014) Paweł Janus, Siem Jan Koopman e Andre Lucas. Long memory dynamics for multivariate dependence under heavy tails. Journal of Empirical Finance, 29:187-206. Citado na pág. 41

Kesten e Spitzer(1984) Harry Kesten e Frank Spitzer. Convergence in distribution of products of random matrices. Probability Theory and Related Fields, 67(4):363-386. Citado na pág. 54

Kingman(1973) John Frank Charles Kingman. Subadditive ergodic theory. The Annals of Probability, 1(6):883-899. Citado na pág. 52

Kogon e Williams(1998) Stephen M. Kogon e Douglas B. Williams. Characteristic function based estimation of stable distribution parameters. Em Robert J. Adler, Raya E. Feldman e Murad S. Taqqu, editors, A practical Guide to Heavy Tailed Data, páginas 311-335. Birkhäuser, Boston. Citado na pág. 17, 26

Kokoszka e Taqqu(1994) Piotr S. Kokoszka e Murad S. Taqqu. Infinite variance stable ARMA processes. Journal of Time Series Analysis, 15(2):203-220. Citado na pág. 24

Kokoszka e Taqqu(1995) Piotr S. Kokoszka e Murad S. Taqqu. Fractional ARIMA with stable innovations. Stochastic Processes and their Applications, 60(1):19-47. Citado na pág. 25

Koopman et al.(2012) Siem Jan Koopman, Andre Lucas e Marcel Scharth. Predicting timevarying parameters with parameter-driven and observation-driven models. Relatório Técnico TI 2012-020/4, Tinbergen Institute, University of Amsterdam. Citado na pág. 4 
Koopman et al.(2016) Siem Jan Koopman, Andre Lucas e Marcel Scharth. Predicting timevarying parameters with parameter-driven and observation-driven modes. Review of Economics and Statistics, 98(1):97-110. Citado na pág. 3, 6

Koutrouvelis(1981) Ioannis A. Koutrouvelis. An iterative procedure for the estimation of the parameters of stable laws. Communications in Statistics - Simulation and Computation, 10(1): 17-28. Citado na pág. 17, 26

Kullback e Leibler(1951) Solomon Kullback e Richard A. Leibler. On information and sufficiency. The Annals of Mathematical Statistic, 22(1):79-86. Citado na pág. 8

Liu e Brorsen(1995) Shi-Miin Liu e B. Wade Brorsen. Maximum likelihood estimation of a GARCH-stable model. Journal of Applied Econometrics, 10:273-285. Citado na pág. 79

Mandelbrot(1963) Benoit Mandelbrot. The variation of certain speculative prices. The Journal of Business, 36(4):394-419. Citado na pág. 2, 16, 39, 40

Mathai et al.(2009) Arakaparampil M. Mathai, Ram Kishore Saxena e Hans J. Haubold. The H-Function: Theory and Applications. Springer, New York. Citado na pág. 27

Matsui e Pawlas(2016) Muneya Matsui e Zbyněk Pawlas. Fractional absolute moments of heavy tailed distributions. Brazilian Journal of Probability and Statistics, 30(2):272-298. Citado na pág. 22

Matsui e Takemura(2006) Muneya Matsui e Akimichi Takemura. Some improvements in numerical evaluation of symmetric stable density and its derivatives. Communications in Statistics Theory and Methods, 35(1):149-172. Citado na pág. 19, 23, 26, 27, 28, 31, 32, 34, 35, 36, 47, 95

McCulloch(1986) J. Huston McCulloch. Simple consistent estimators of stable distribution parameters. Communications in Statistics - Simulation and Computation, 15(4):1109-1136. Citado na pág. 17,26

Mittnik e Paolella(2000) Stefan Mittnik e Marc S. Paolella. Conditional density and Value-atRisk prediction of Asian currency exchange rates. Journal of Forecasting, 19(4):313-333. Citado na pág. 82

Mittnik et al.(1998) Stefan Mittnik, Svetlozar T. Rachev e Marc S. Paolella. Stable Paretian modeling in Finance: some empirical and theoretical aspects. Em Robert J. Adler, Raya E. Feldman e Murad S. Taqqu, editors, A practical Guide to Heavy Tailed Data, páginas 79-110. Birkhäuser, Boston. Citado na pág. 40

Mittnik et al.(2002) Stefan Mittnik, Marc S. Paolella e Svetlozar T. Rachev. Stationarity of stable power-GARCH processes. Journal of Econometrics, 106(1):97-107. Citado na pág. 2, 40, 43, 50, 52

Morettin(2011) Pedro Alberto Morettin. Econometria Financeira: Um Curso em Séries Temporais Financeiras. Edgard Blücher, São Paulo, $2^{\mathrm{a}}$ ed. edição. Citado na pág. 1, 3, 6, 41, 42, 54, 57, 58,82

Nelson(1991) Daniel B. Nelson. Conditional Heteroskedasticity in Asset Returns: A New Approach. Econometrica, 59(2):347-370. Citado na pág. 39

Nelson e Cao(1992) Daniel B. Nelson e Charles Q. Cao. Inequality constraints in the univariate GARCH model. Journal of Business \& Economic Statistics, 10(2):229-235. Citado na pág. 42, 49

Nelson e Foster(1994) Daniel B. Nelson e Dean P. Foster. Asymptotic filtering theory for univariate ARCH models. Econometrica, 62(1):1-41. Citado na pág. 4

Nobel(2017) Nobel. All Prizes in Economic Sciences, 2017. URL https://www.nobelprize.org/ nobel_prizes/economic-sciences/laureates/. Último acesso em 05/11/2017. Citado na pág. 3 
Nolan(2001) John P. Nolan. Maximum likelihood estimation and diagnostics for stable distributions. Em Ole E. Barndorff-Nielsen, Thomas Mikosch e Sidney I. Resnic, editors, Lévy Processes: Theory and Applications, páginas 379-400. Birkhäuser, Boston. Citado na pág. 2, 15, 17

Nolan(2017) John P. Nolan. Stable Distributions Models for Heavy Tailed Data. Birkhäuser, Boston. URL http://academic2.american.edu/ jpnolan/stable/chap1.pdf. Livro em desenvolvimento. Capítulo 1 disponível. Último acesso em 05/11/2017. Citado na pág. 18, 19, 22, 23, 24, 25

Nolan(1997) John P. Nolan. Numerical computation of stable densities and distribution functions. Communications in Statistics - Stochastic Models, 13(4):759-774. Citado na pág. 19, 23, 26, 27, 28, 47

Panorska et al.(1995) Ania K. Panorska, Stefan Mittnik e Svetlozar T. Rachev. Stable GARCH models for financial time series. Applied Mathematics Letters, 8(5):33-37. Citado na pág. 2, 15, 40, 42

Piessens et al.(1983) Robert Piessens, Elise de Doncker-Kapenga, Christoph W. Überhuber e David K. Kahaner. Quadpack: a Subroutine Package for Automatic Integration. Springer, Berlin Heidelberg. Citado na pág. 30

Pollard(1946) Harry Pollard. The representation of $e^{-x^{\lambda}}$ as a Laplace integral. Bulletin of the American Mathematical Society, 52(10):908-910. Citado na pág. 30

Press(1972) James S. Press. Estimation in univariate and multivariate stable distributions. Journal of the American Statistical Association, 67(340):842-846. Citado na pág. 17, 26

Rachev et al.(2005) Svetlozar T. Rachev, Stoyan Stoyanov, Almira Biglova e Frank J. Fabozzi. An empirical examination of daily stock return distributions for U.S. stocks. Em Daniel Baier, Reinhold Decker e Lars Schmidt-Thieme, editors, Data Analysis and Decision Support, páginas 269-281. Springer, Berlin Heidelberg. Citado na pág. 79

Rao(1973) Calyampudi Radhakrishna Rao. Linear Statistical Inference and Its Applications. Wiley, New York. Citado na pág. 57

Rosadi e Deistler(2011) Dedi Rosadi e Manfred Deistler. Estimating the codifference function of linear time series models with infinite variance. Metrika, 73(3):395-429. Citado na pág. 58

Ruggiero e Lopes(1997) Márcia A. Gomes Ruggiero e Vera Lúcia da Rocha Lopes. Cálculo Numérico: Aspectos Teóricos e Computacionais. Makron Books do Brasil. Citado na pág. 30

Russell e Engle(2005) Jeffrey R. Russell e Robert F. Engle. A discrete-state continuous-time model of financial transactions prices and times. Journal of Business 83 Economic Statistics, 23 (2):166-180. Citado na pág. 6

Samorodnitsky e Taqqu(1994) Gennady Samorodnitsky e Murad S. Taqqu. Stable Non Gaussian Random Process: Stochastic models with infinite variance. Chapman and Hall, New York. Citado na pág. $2,17,18,21,26$

Sousa(2013) Thiago do Rêgo Sousa. Modelos Combinados ARMA-GARCH Governados por Distribuições Estáveis. Dissertação de Mestrado, Departamento de Estatística, Universidade de Brasília. Citado na pág. 27

Stein et al.(2016) Josiane Stein, Silvia Regina Costa Lopes e Ary Vasconcelos Medino. Continuous processes derived from the solution of generalized Langevin equation: theoretical properties and estimation. Journal of Statistical Computation and Simulation, 86(14):2819-2845. Citado na pág. 25 
Taylor(1980) Stephen J. Taylor. Conjectured models for trends in financial prices, tests and forecasts. Journal of the Royal Statistical Society. Series A (General), 143(3):338-362. Citado na pág. 3

West e Harrison(1997) Mike West e Jeff Harrison. Bayesian Forecasting and Dynamic Models. Springer, New York. Citado na pág. 1

Zivot(2009) Eric Zivot. Practical issues in the analysis of univariate GARCH. Em Torben Gustav Andersen, Richard A. Davis, Jens-Peter Kreiss e Thomas V. Mikosch, editors, Handbook of Financial Time Series, páginas 113-155. Springer, Berlin Heidelberg. Citado na pág. 42, 82

Zolotarev(1986) Vladimir M. Zolotarev. One-dimensional Stable Distributions. "Translations of mathematical monographs" (vol. 65), American Mathematical Society. Citado na pág. 16, 17, 18, $19,23,26,27,29,30,47$ 